\title{
Protecting-Group-Mediated Diastereoselective Synthesis of
}

\section{C4'-Methylated Uridine Analogs and their Activity \\ against the Human Respiratory Syncytial Virus}

Christoph Köllmann, Svenja M. Sake, Peter G. Jones,

Thomas Pietschmann, and Daniel B. Werz*

\section{Supporting Information}

Table of Contents

1. Spectroscopic Data

L-Lyxose Series

S2

L-Lyxose Spirocyclopropane Series

S38

D-Ribose Series

S53

2. X-ray Structure Determinations

574

3. Antiviral Investigations

S115

4. References

S116 
1. Spectroscopic Data

L-Lyxose Series 

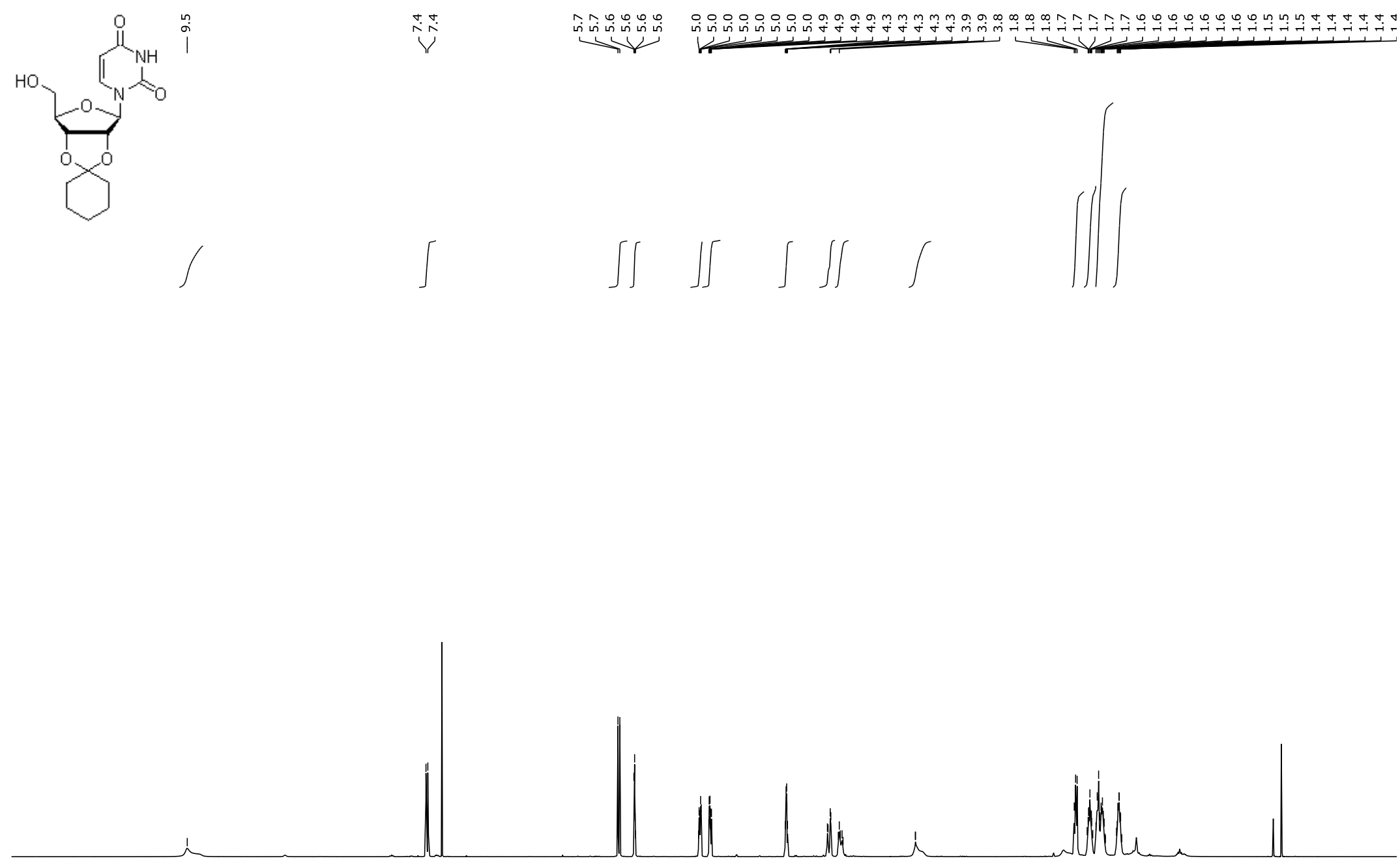

\begin{tabular}{llllllllllllllllllllllllllllll}
\hline 1.0 & 10.5 & 10.0 & 9.5 & 9.0 & 8.5 & 8.0 & 7.5 & 7.0 & 6.5 & 6.0 & 5.5 & 5.0 & 4.5 & 4.0 & 3.5 & 3.0 & 2.5 & 2.0 & 1.5 & 1.0 & 0.5 & 0.0 & -0.5 & -1
\end{tabular}

Figure S1. ${ }^{1} \mathrm{H}$ NMR $\left(500 \mathrm{MHz}, \mathrm{CDCl}_{3}\right)$ spectrum of compound $\mathbf{1 a}$. 


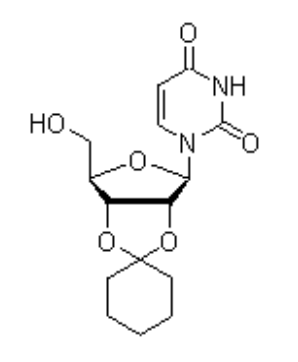

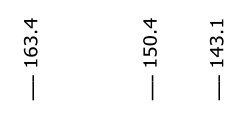

$\stackrel{\text { N }}{\stackrel{\text { ñ }}{-1}}$

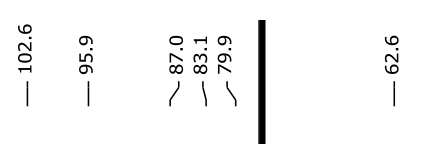
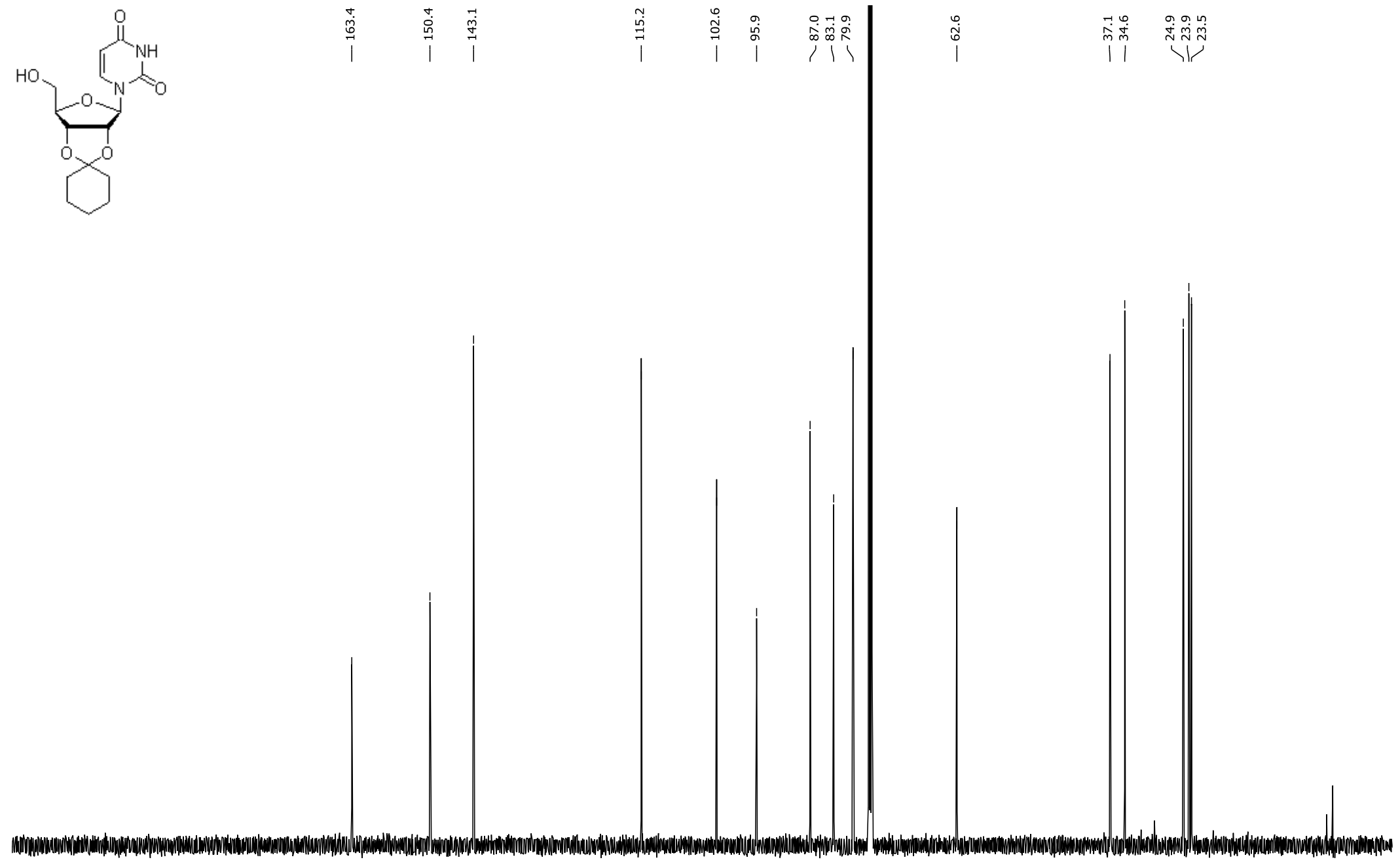

20
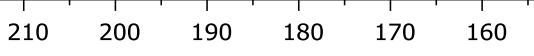

150

130

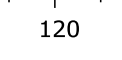

$110 \quad 100$

Figure S2. $\left.{ }^{13} \mathrm{C} \mathrm{NMR} \mathrm{(126} \mathrm{MHz,} \mathrm{CDCl}_{3}\right)$ spectrum of compound $\mathbf{1 a}$. 


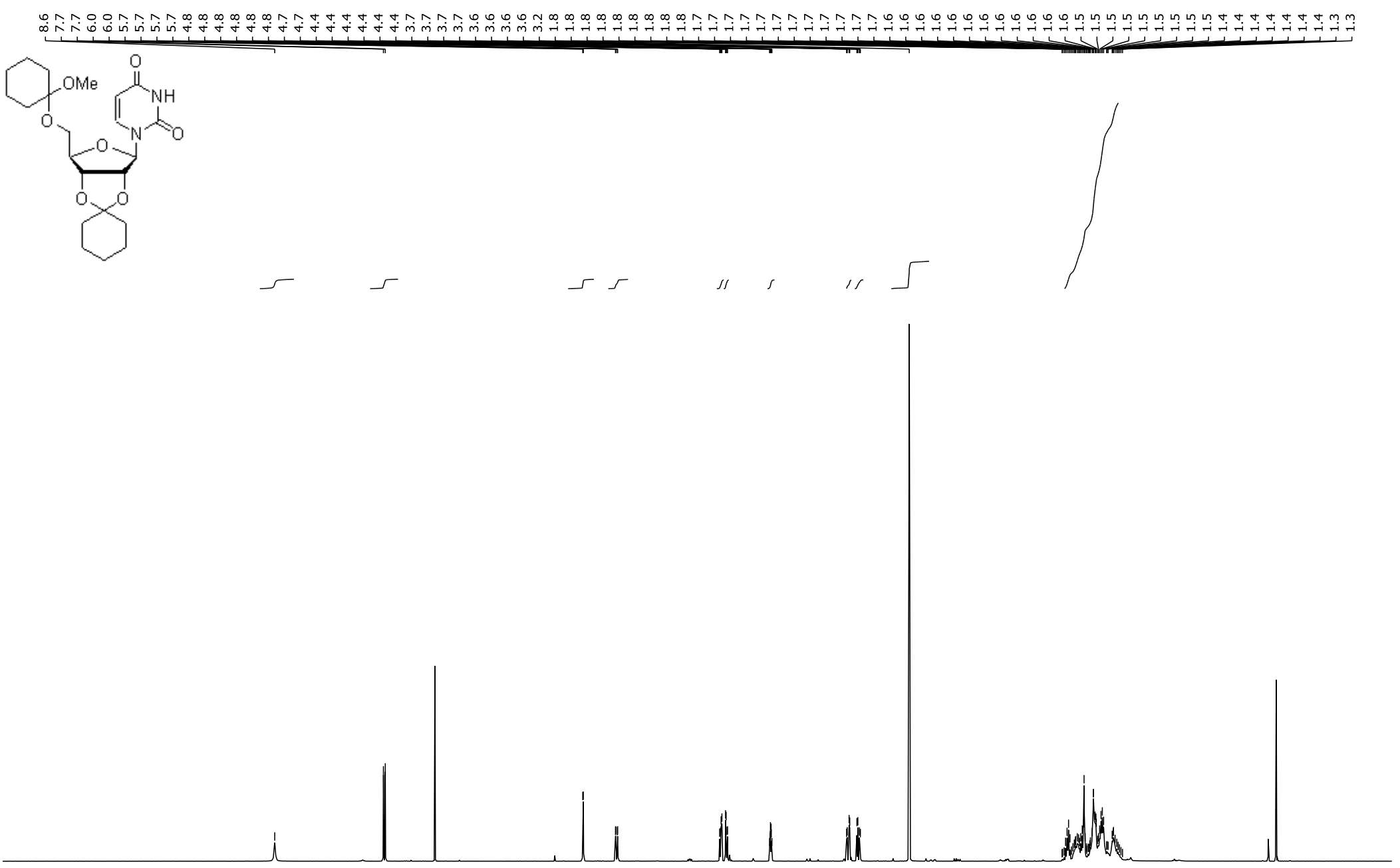

\begin{tabular}{lllllllll}
\hline .0 & 10.5 & 10.0 & 9.5 & 9.0 & 8.5 & 8.0 & 7.5 & 7.0
\end{tabular}

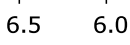

$5.5 \quad 5.0$

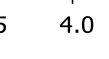

3.5

$3.0 \quad 2.5$ $2.0 \quad 1.5$

$\begin{array}{llllll}1.0 & 0.5 & 0.0 & -0.5 & -1\end{array}$

Figure S3. ${ }^{1} \mathrm{H}$ NMR (500 MHz, $\left.\mathrm{CDCl}_{3}\right)$ spectrum of compound $1 \mathrm{c}$. 


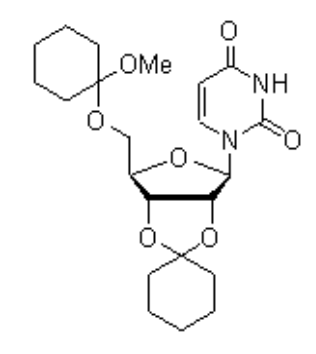

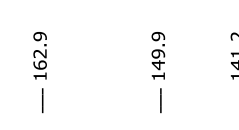

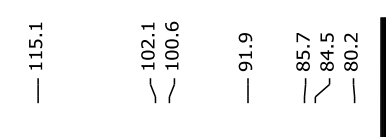

|

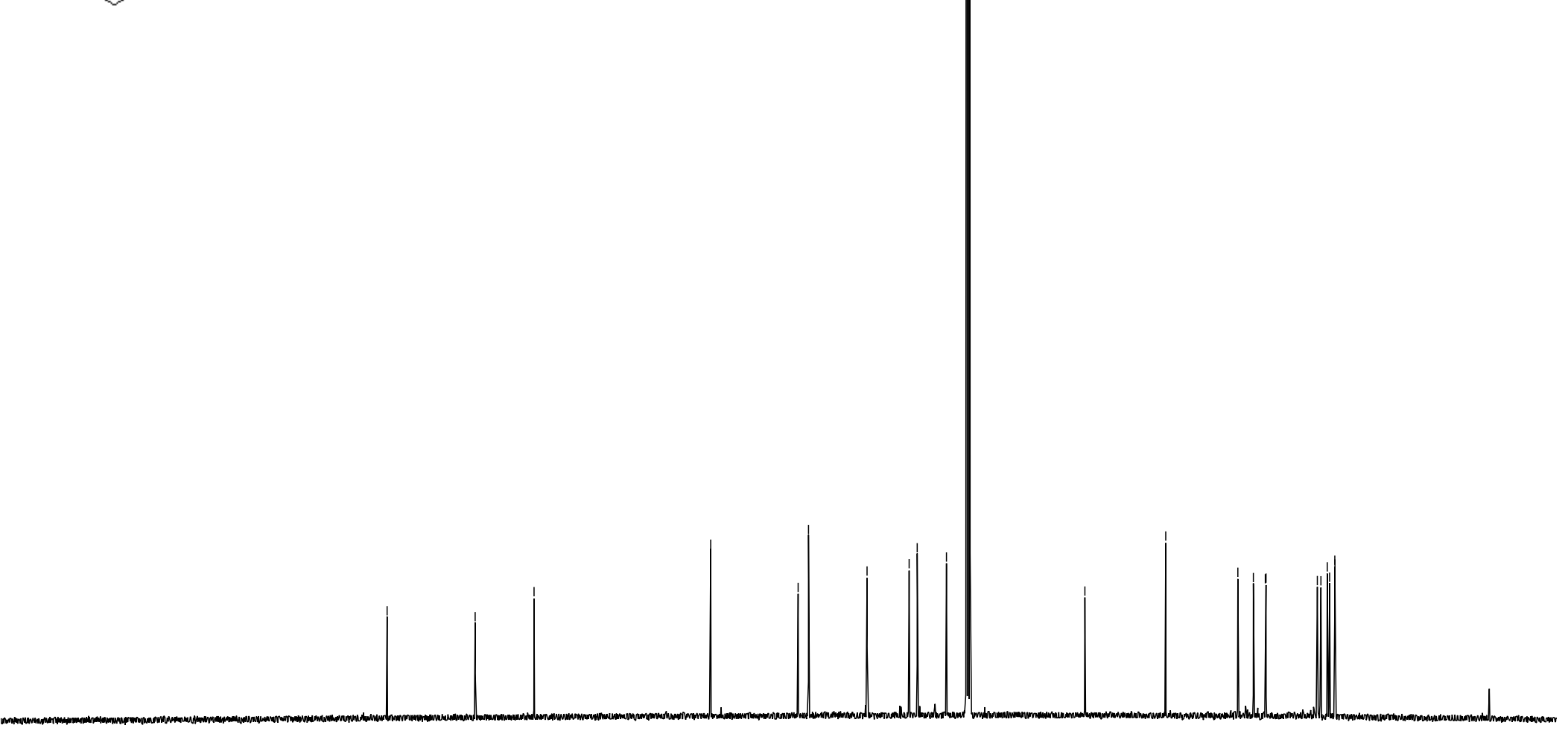

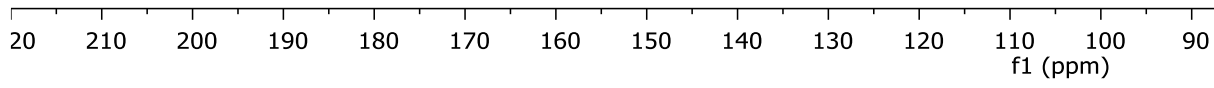

Figure S4. ${ }^{13} \mathrm{C}$ NMR (126 MHz, $\left.\mathrm{CDCl}_{3}\right)$ spectrum of compound $\mathbf{1 c}$. 

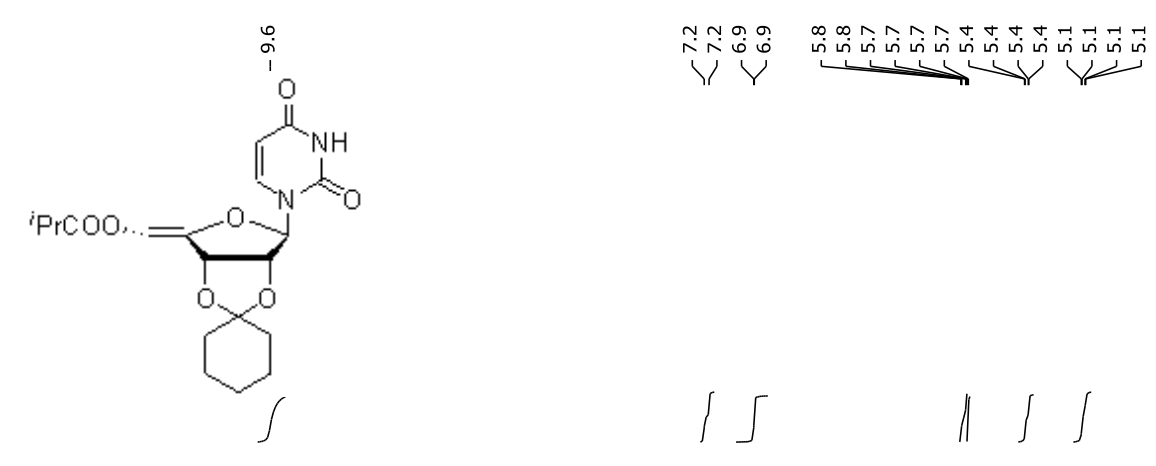

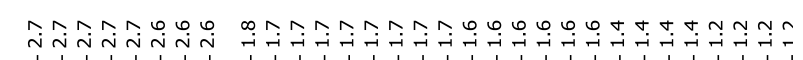
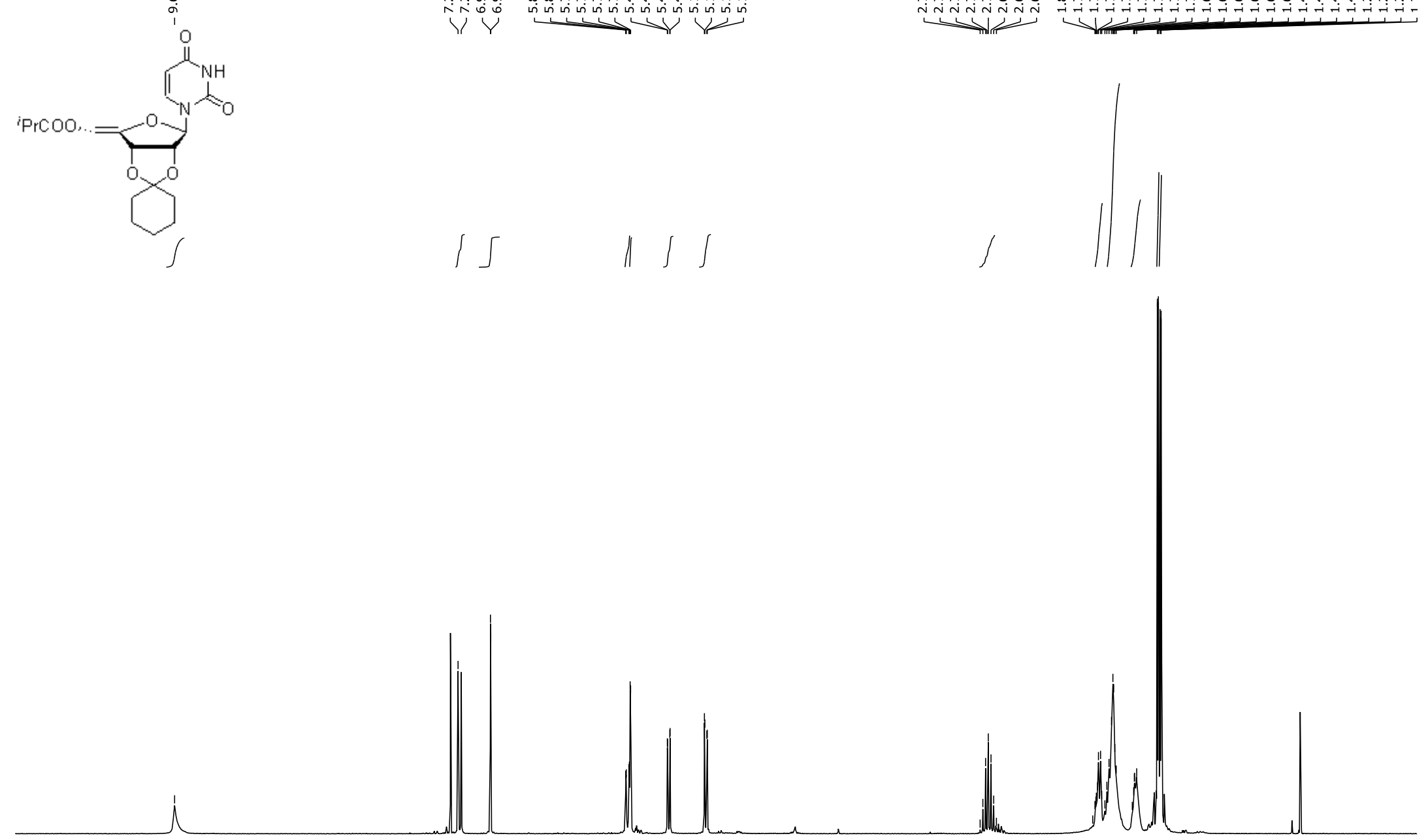

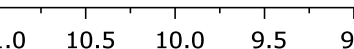
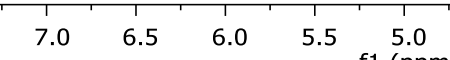

f1 $(\mathrm{ppm})$

Figure S5. ${ }^{1} \mathrm{H} \mathrm{NMR}\left(300 \mathrm{MHz}, \mathrm{CDCl}_{3}\right)$ spectrum of compound 2a. 

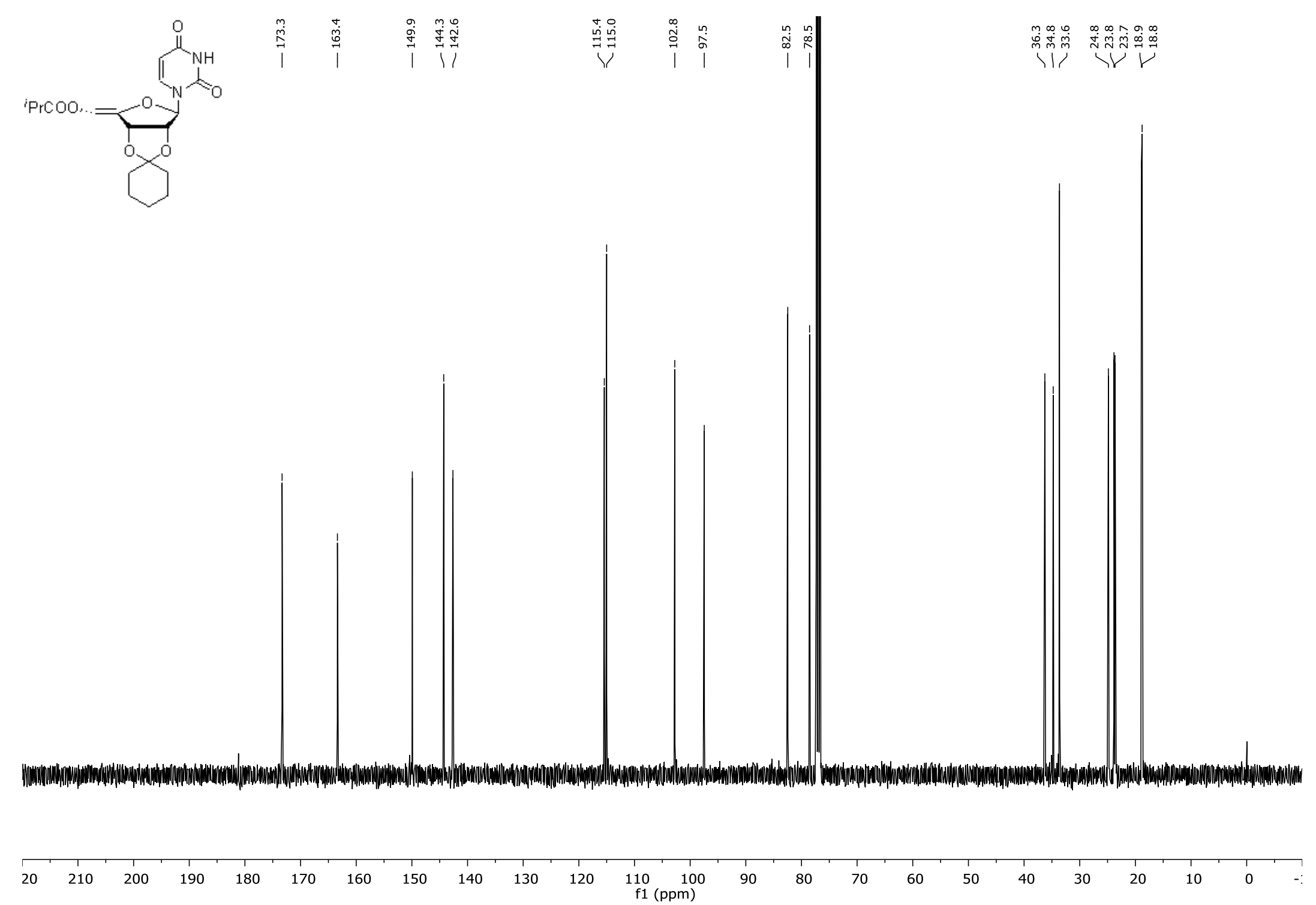

Figure S6. ${ }^{13} \mathrm{C}$ NMR (101 MHz, $\left.\mathrm{CDCl}_{3}\right)$ spectrum of compound 2a. 


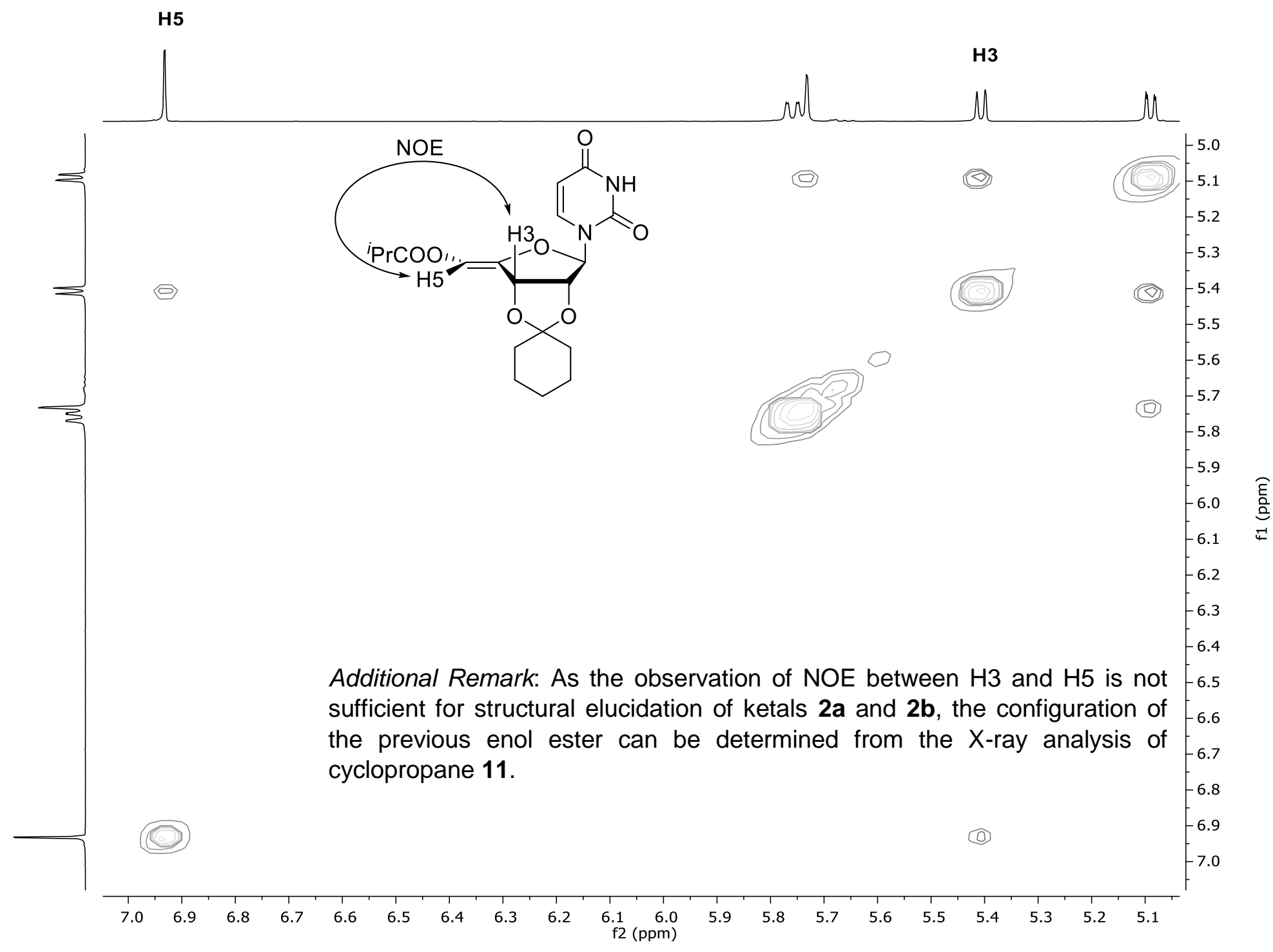

Figure S7. Partial NOESY (400 MHz, $\left.\mathrm{CDCl}_{3}\right)$ of compound 2a. 

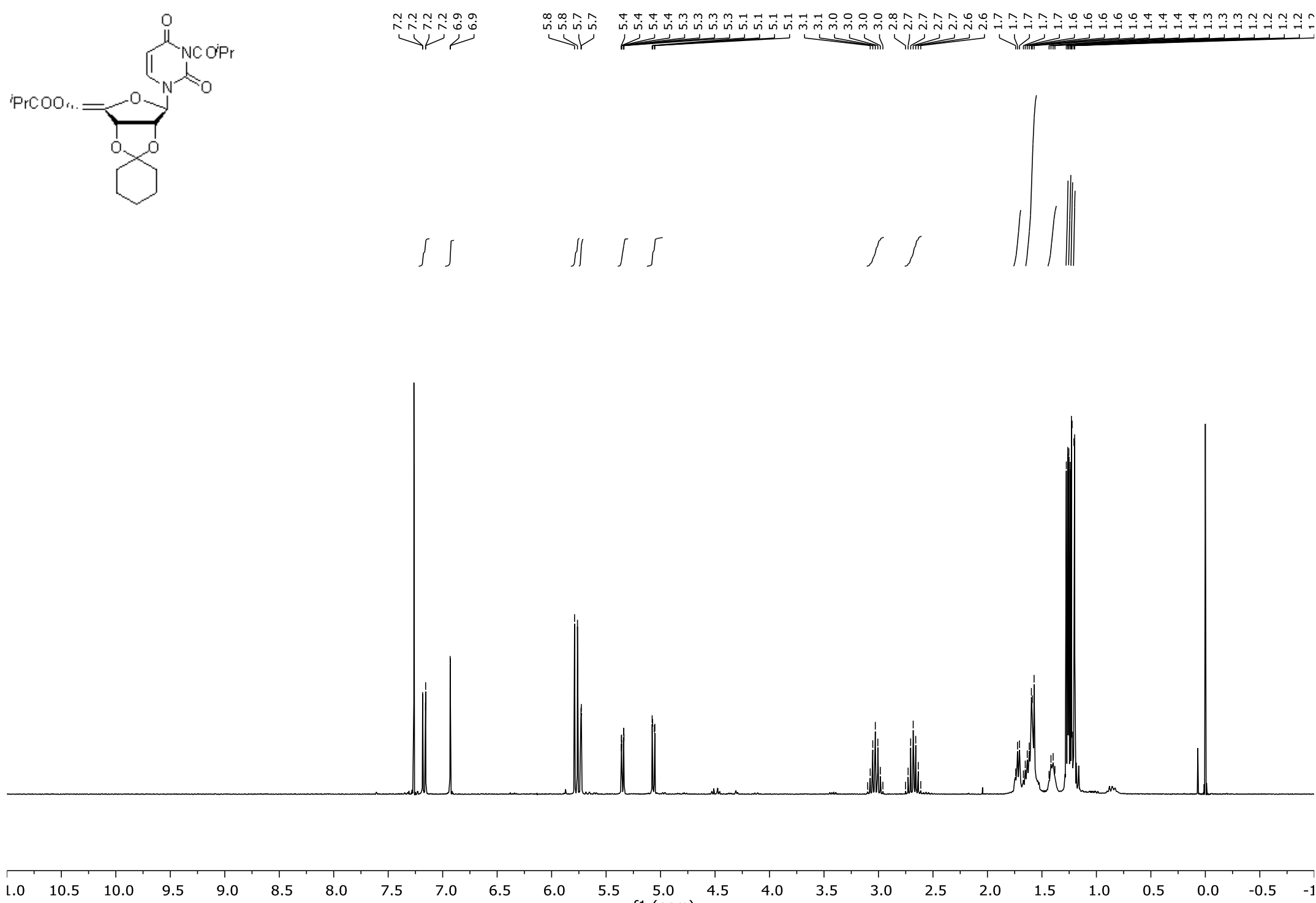

6.0

$5.5 \quad 5.0$

$\begin{array}{llllllllllll}4.5 & 4.0 & 3.5 & 3.0 & 2.5 & 2.0 & 1.5 & 1.0 & 0.5 & 0.0 & -0.5 & -1\end{array}$

Figure S8. ${ }^{1} \mathrm{H} \mathrm{NMR}\left(300 \mathrm{MHz}, \mathrm{CDCl}_{3}\right)$ spectrum of compound $2 \mathrm{c}$. 

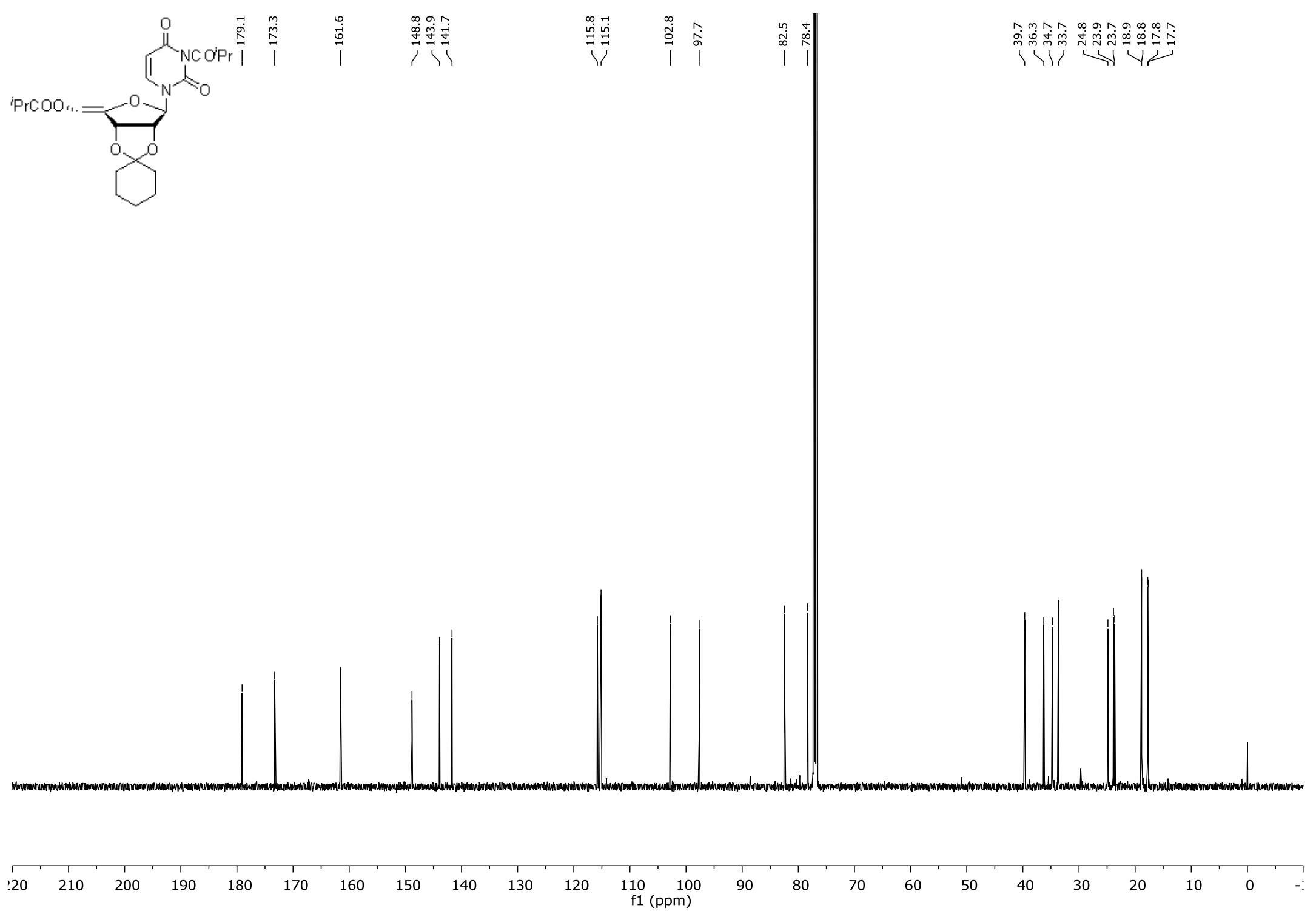

Figure S9. ${ }^{1} \mathrm{H}$ NMR (126 MHz, $\mathrm{CDCl}_{3}$ ) spectrum of compound $2 \mathrm{c}$. 

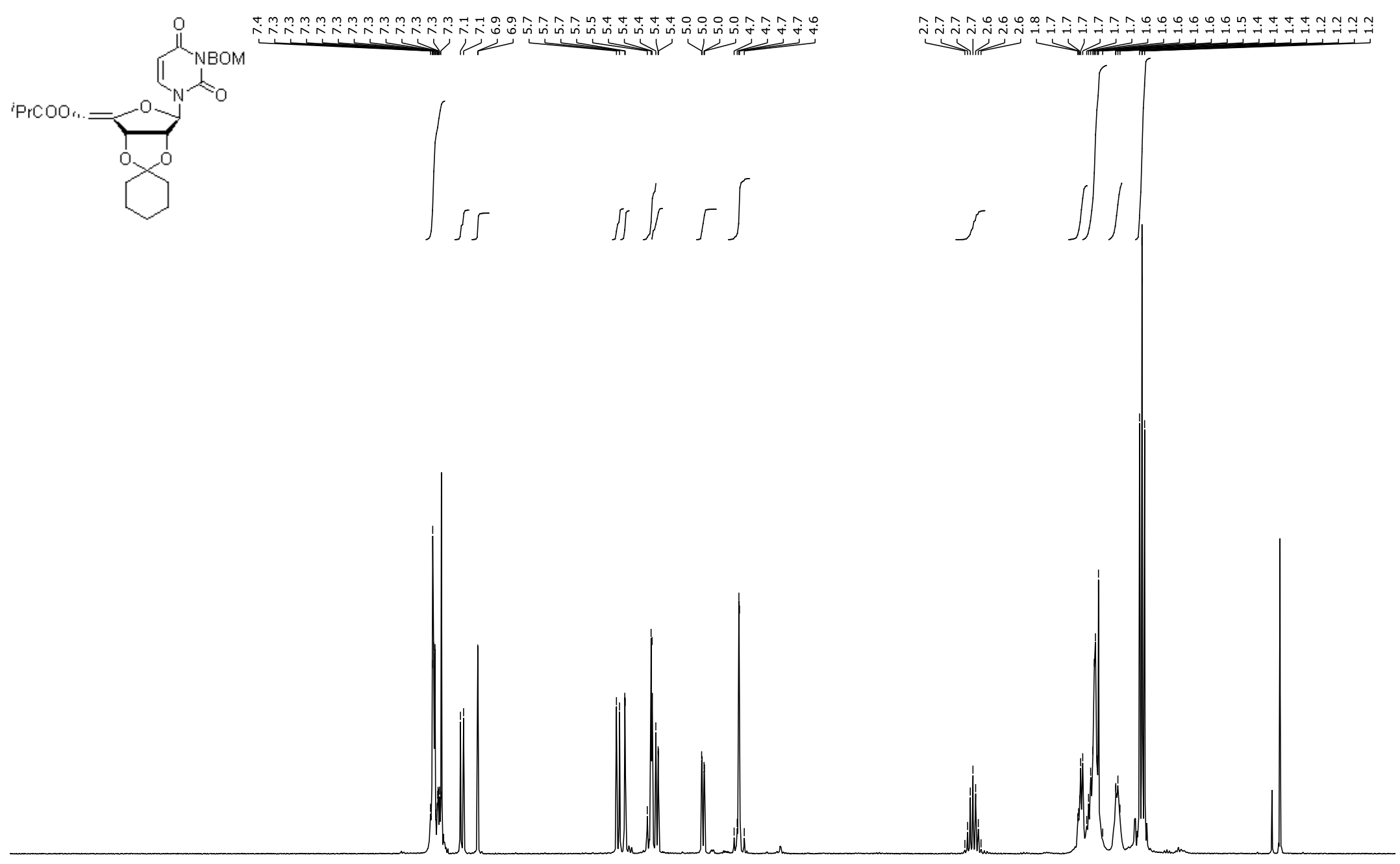

$\begin{array}{llllllll}10.5 & 10.0 & 9.5 & 9.0 & 8.5 & 8.0 & 7.5 & 7.0\end{array}$

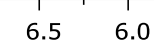

$5.5 \stackrel{5.0}{5}$

(ppm)

Figure S10. ${ }^{1} \mathrm{H} \mathrm{NMR}\left(300 \mathrm{MHz}, \mathrm{CDCl}_{3}\right)$ spectrum of compound 3 a. 

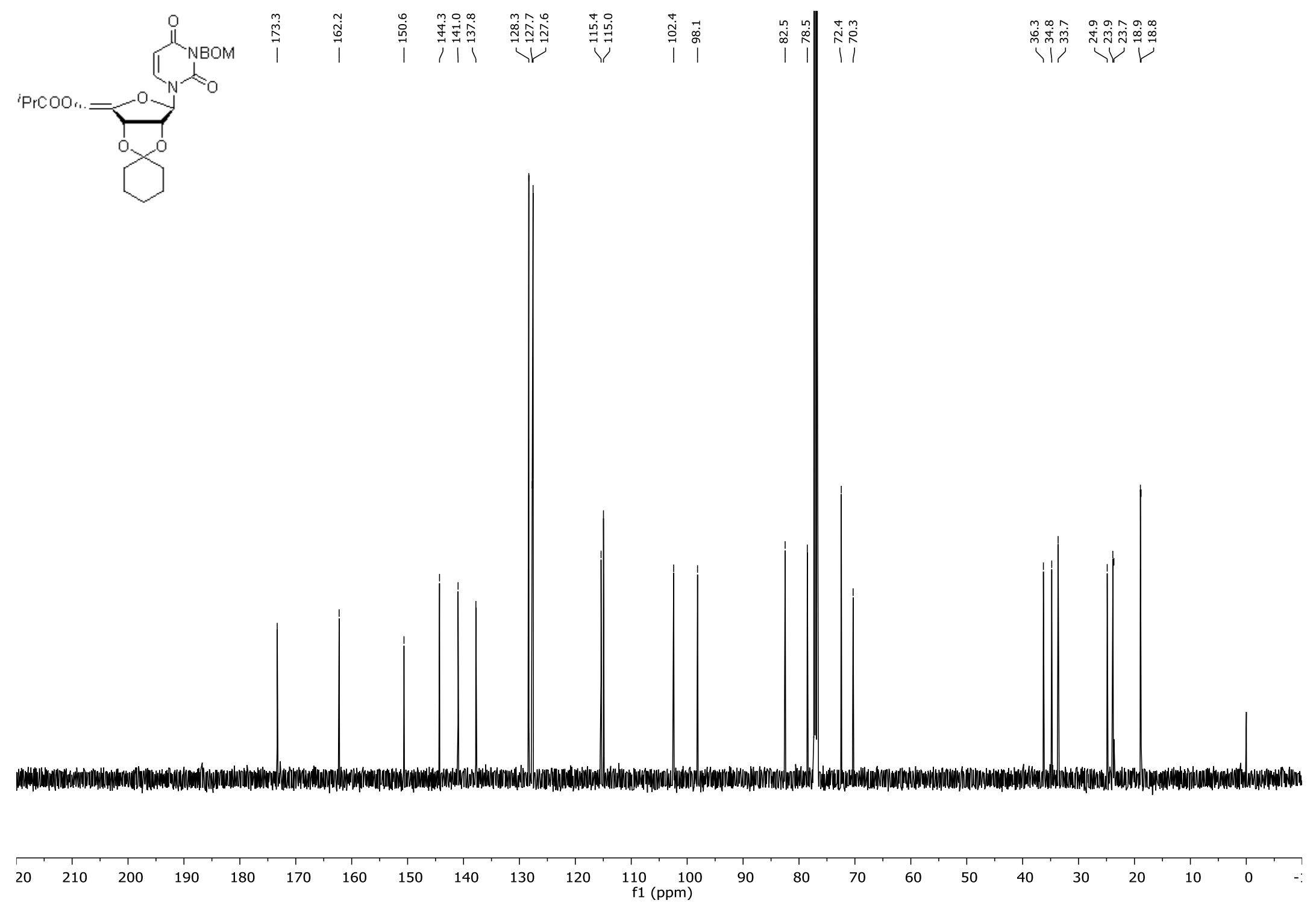

Figure S11. ${ }^{1} \mathrm{H} \mathrm{NMR}\left(126 \mathrm{MHz}, \mathrm{CDCl}_{3}\right)$ spectrum of compound $\mathbf{3 a}$. 

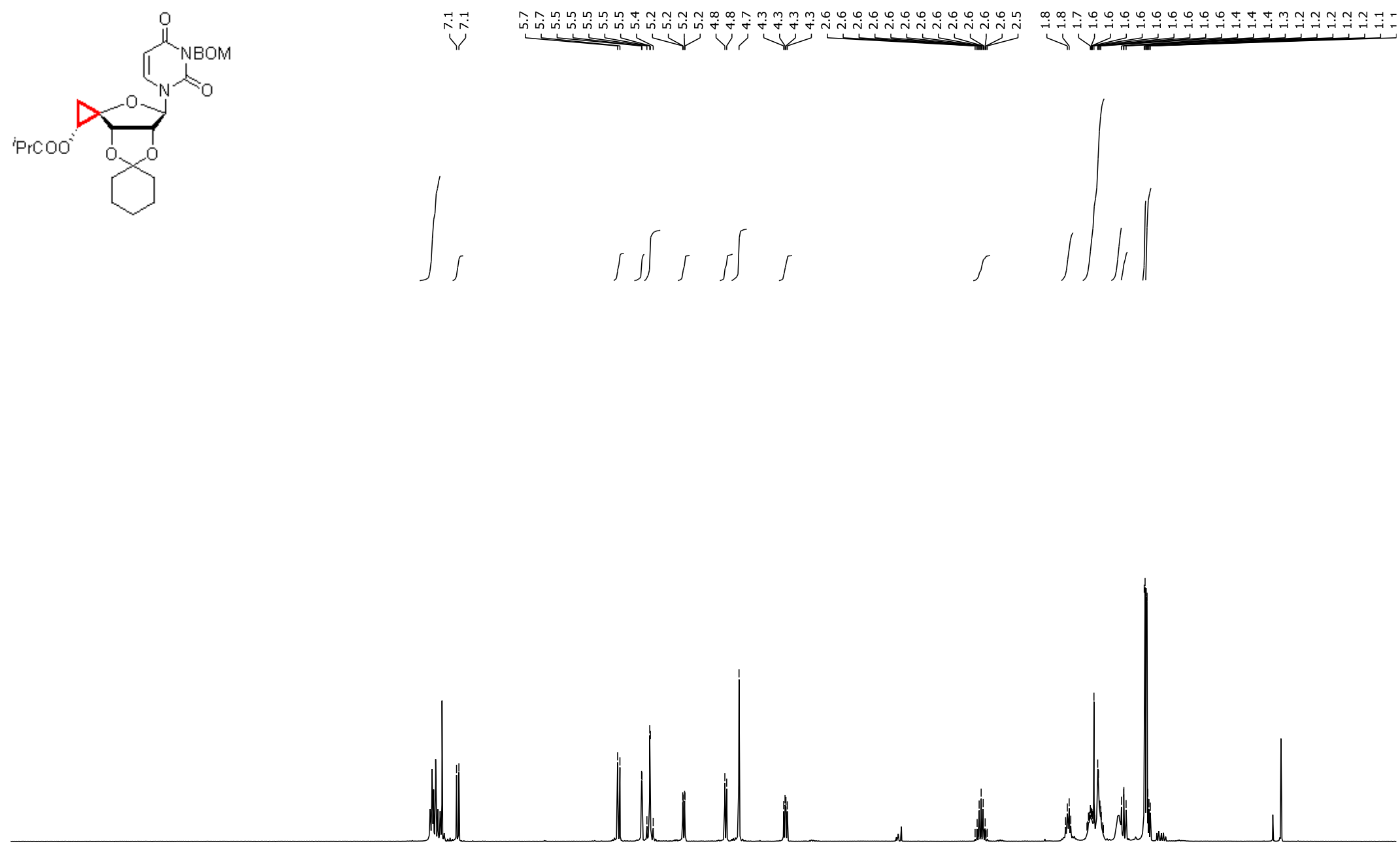

$1.0 \quad 10.5 \quad 10.0 \quad 9.5 \quad 9.0$ $\begin{array}{lll}8.5 & 8.0 & 7.5\end{array}$

Figure S12. ${ }^{1} \mathrm{H}$ NMR (400 MHz, $\left.\mathrm{CDCl}_{3}\right)$ spectrum of compound $\mathbf{4 a}$. 


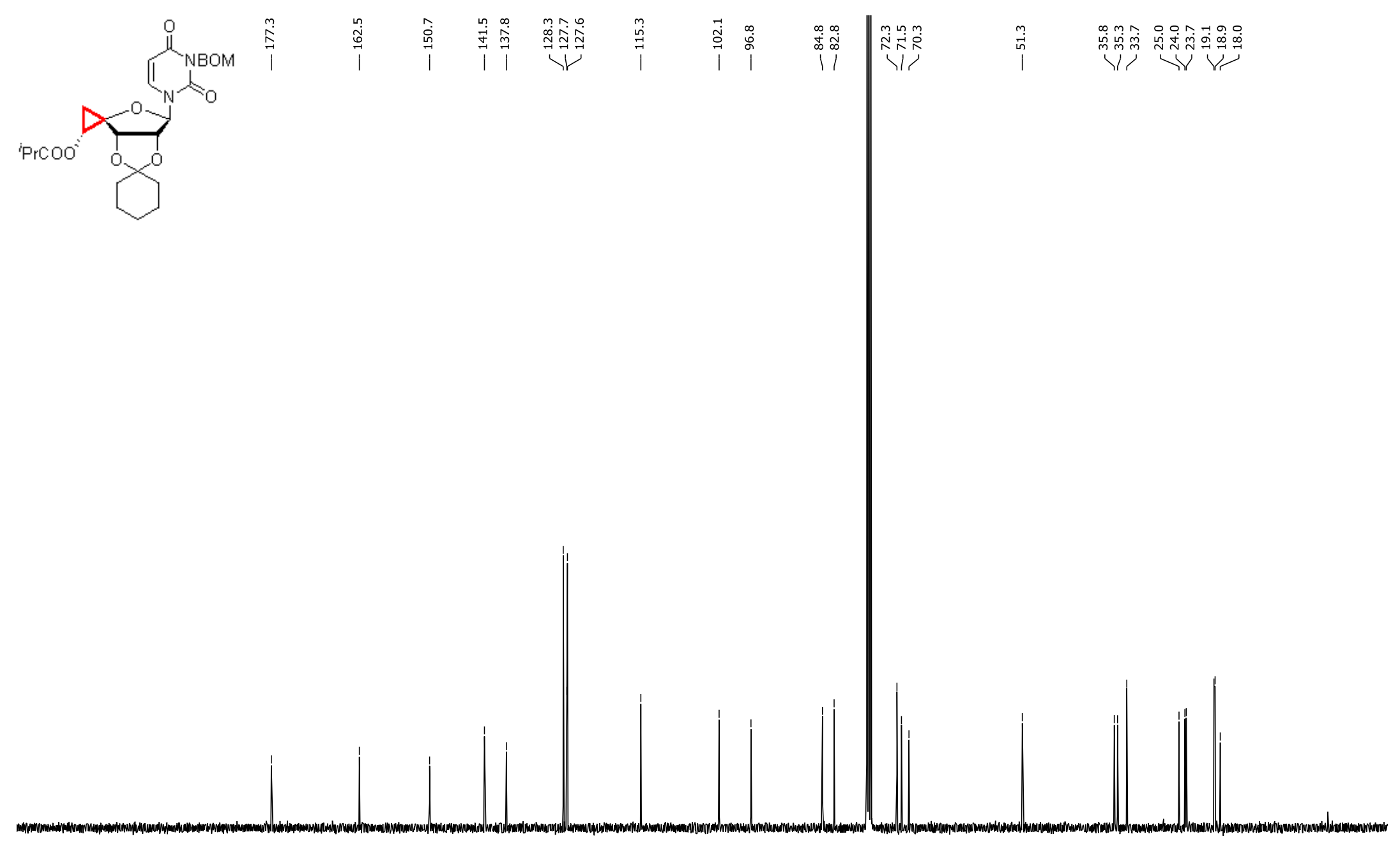$$
20
$$

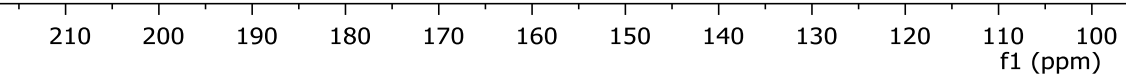

Figure $\mathbf{S 1 3} \cdot{ }^{13} \mathrm{C}$ NMR $\left(101 \mathrm{MHz}, \mathrm{CDCl}_{3}\right)$ spectrum of compound $\mathbf{4 a}$. 


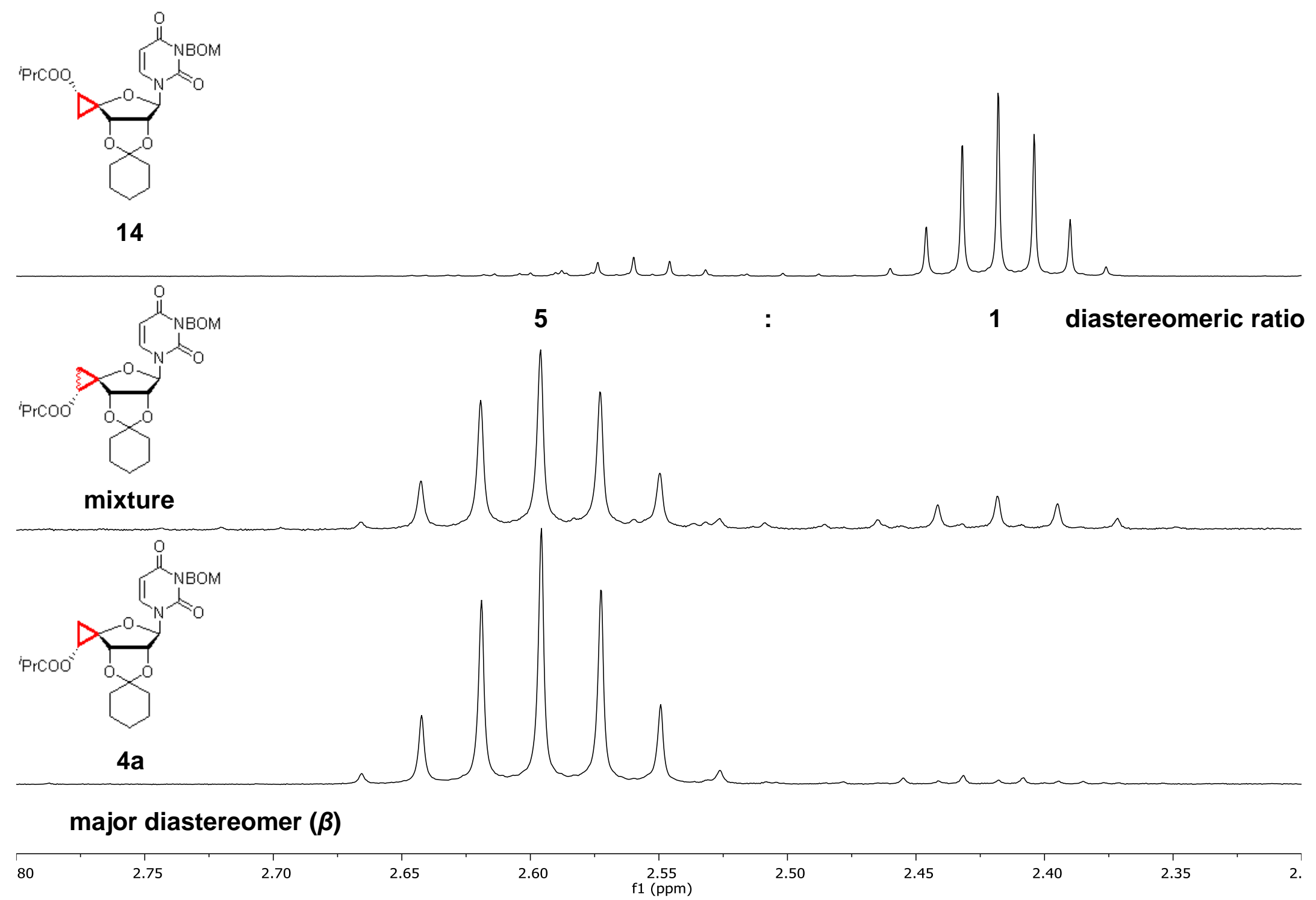

Figure S14. ${ }^{1} \mathrm{H}$ NMR $\left(400 \mathrm{MHz}, \mathrm{CDCl}_{3}\right)$ Determination of the diastereomeric ratio of product mixture 4a. 

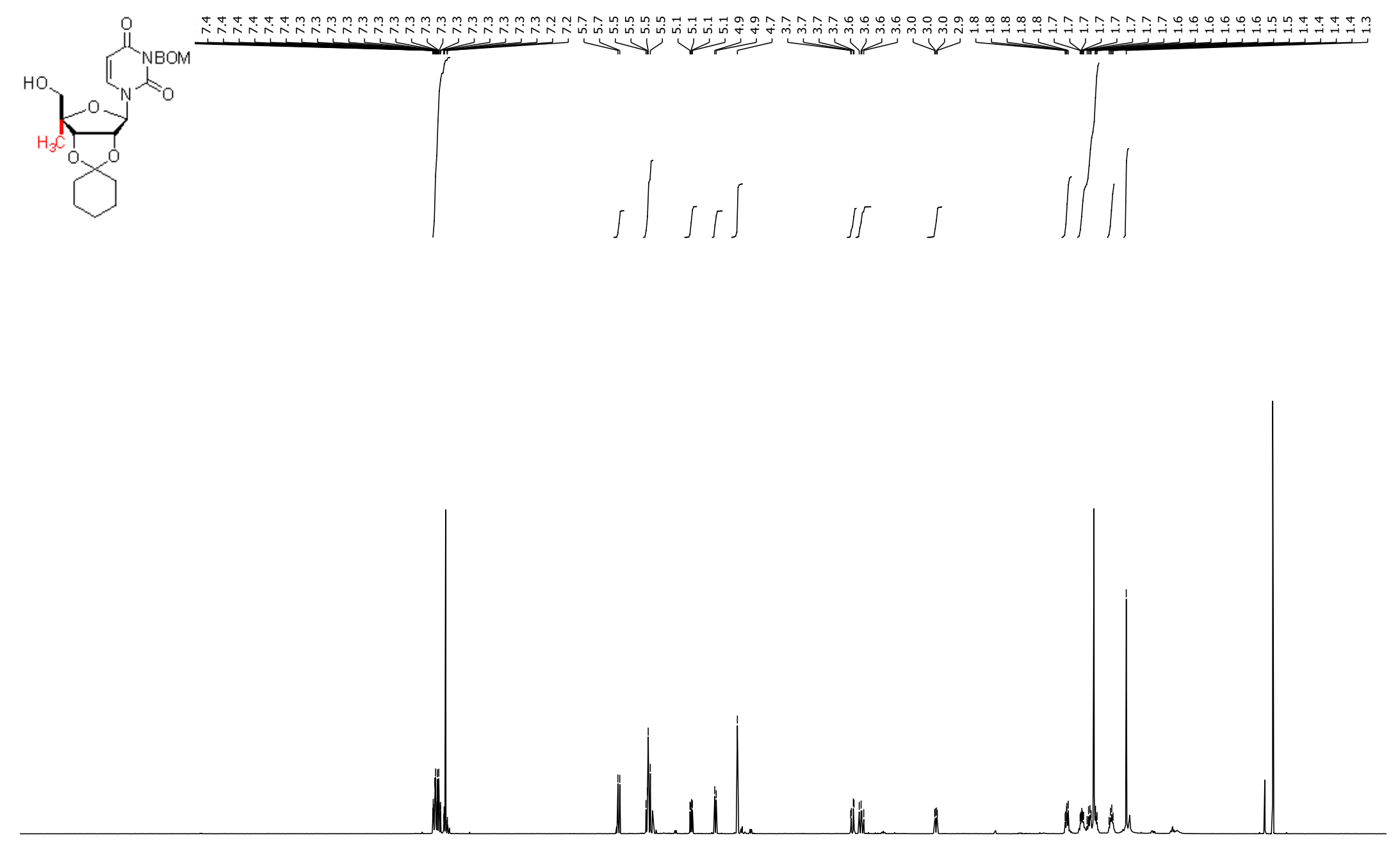

$\begin{array}{lll}1.0 & 10.5 & 10.0\end{array}$

Figure S15. ${ }^{1} \mathrm{H} \mathrm{NMR}\left(500 \mathrm{MHz}, \mathrm{CDCl}_{3}\right)$ spectrum of compound $\mathbf{5 a}$. 


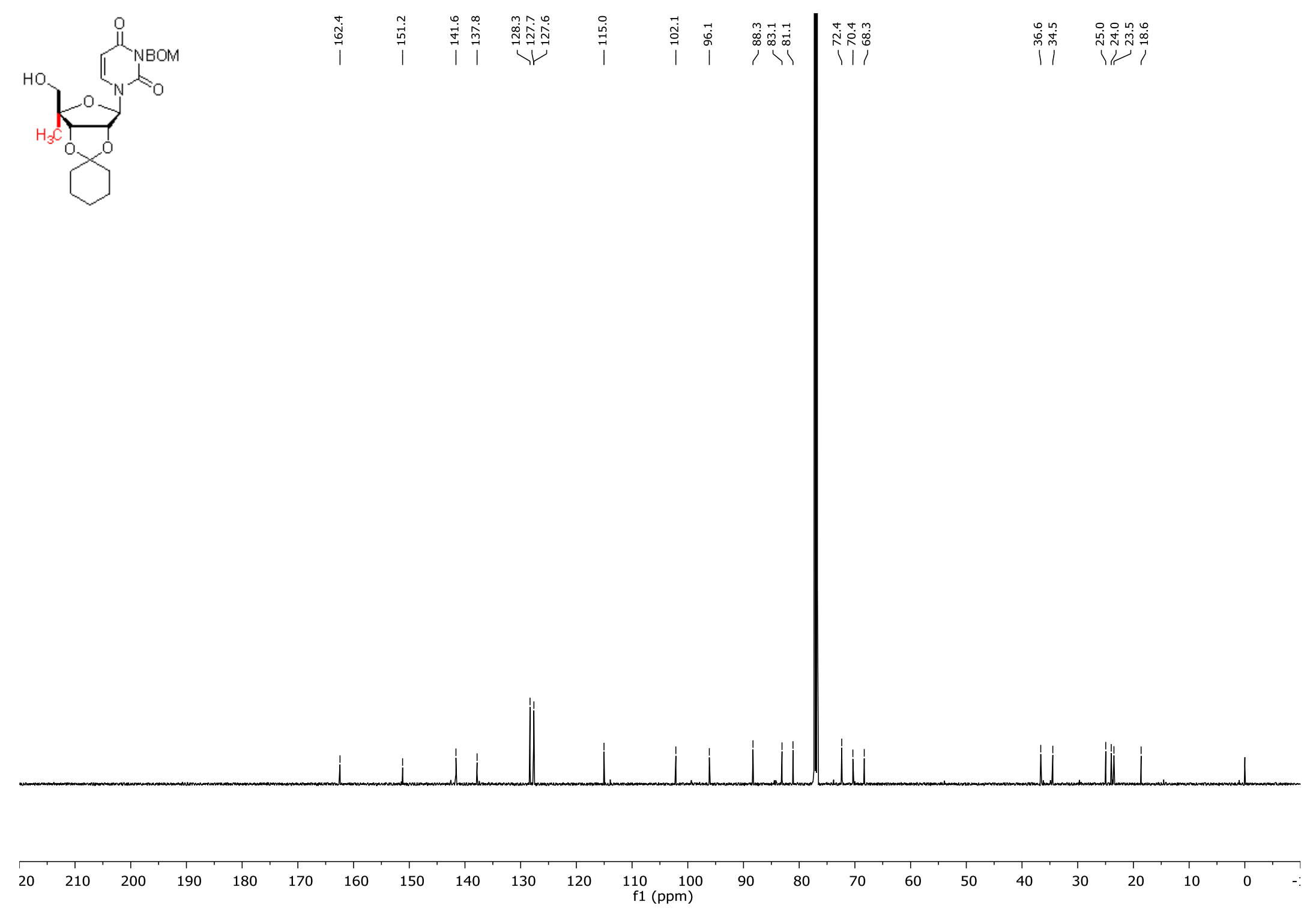

Figure S16. ${ }^{1} \mathrm{H} \mathrm{NMR}\left(126 \mathrm{MHz}, \mathrm{CDCl}_{3}\right)$ spectrum of compound $\mathbf{5 a}$. 


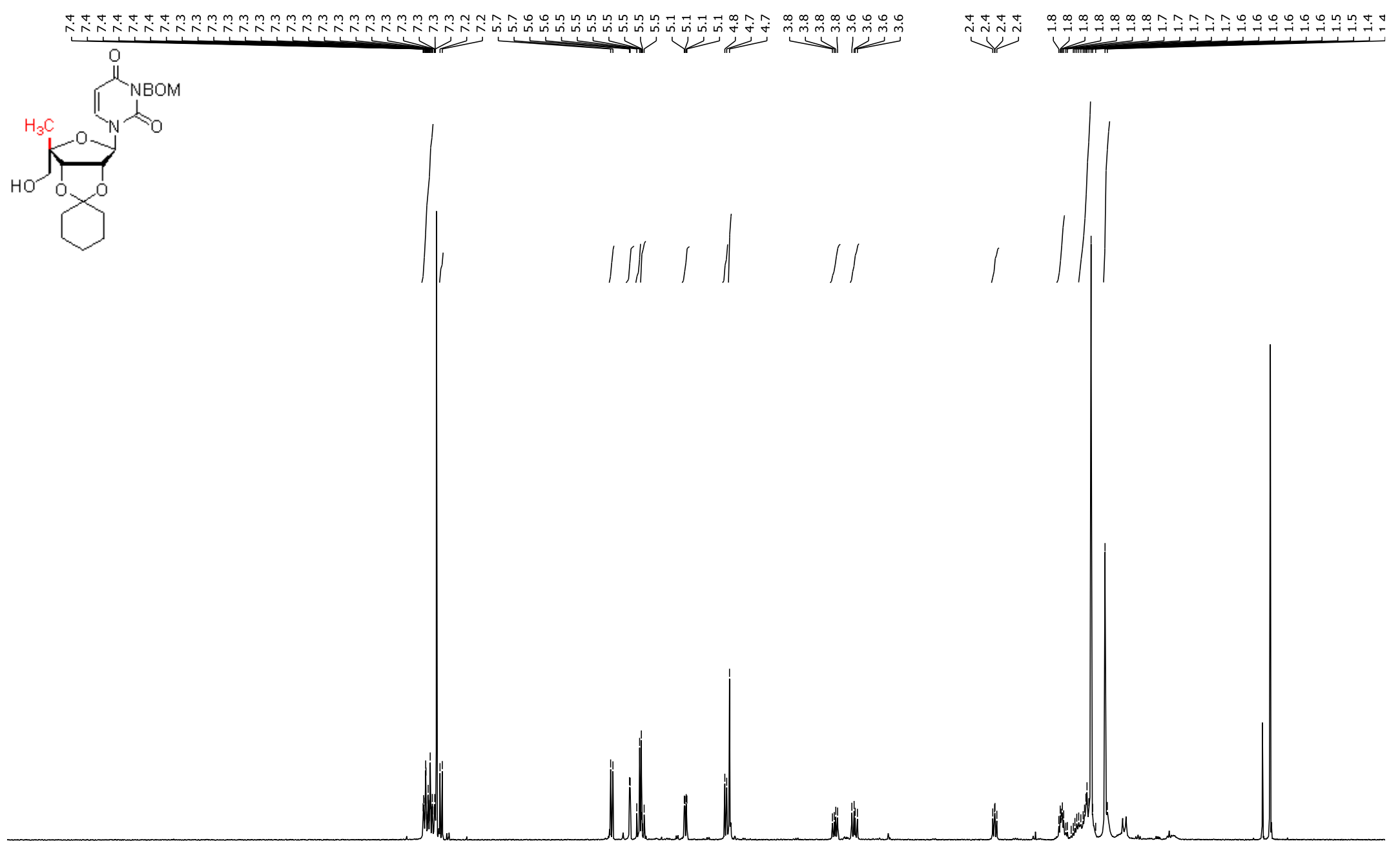

$1.0+10.5+10.0$

Figure S17. ${ }^{1} \mathrm{H}$ NMR (400 MHz, $\mathrm{CDCl}_{3}$ ) spectrum of compound 5. 

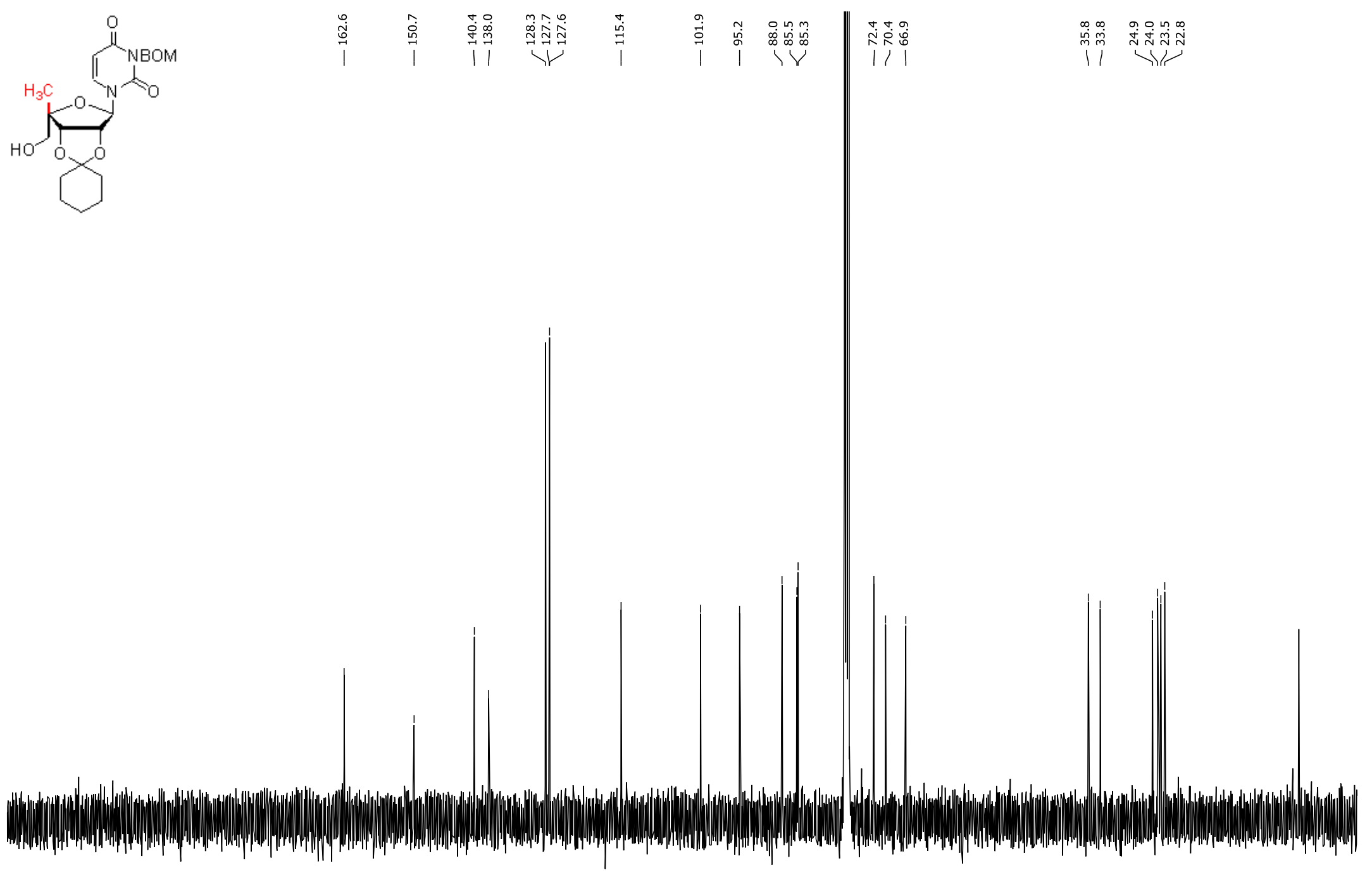

$\begin{array}{llllllllllllll}20 & 210 & 200 & 190 & 180 & 170 & 160 & 150 & 140 & 130 & 120 & \begin{array}{c}110 \\ \mathrm{f} 1(\mathrm{ppm})\end{array} & 90\end{array}$

Figure S18. ${ }^{13} \mathrm{C}$ NMR (101 MHz, $\left.\mathrm{CDCl}_{3}\right)$ spectrum of compound 5 . 


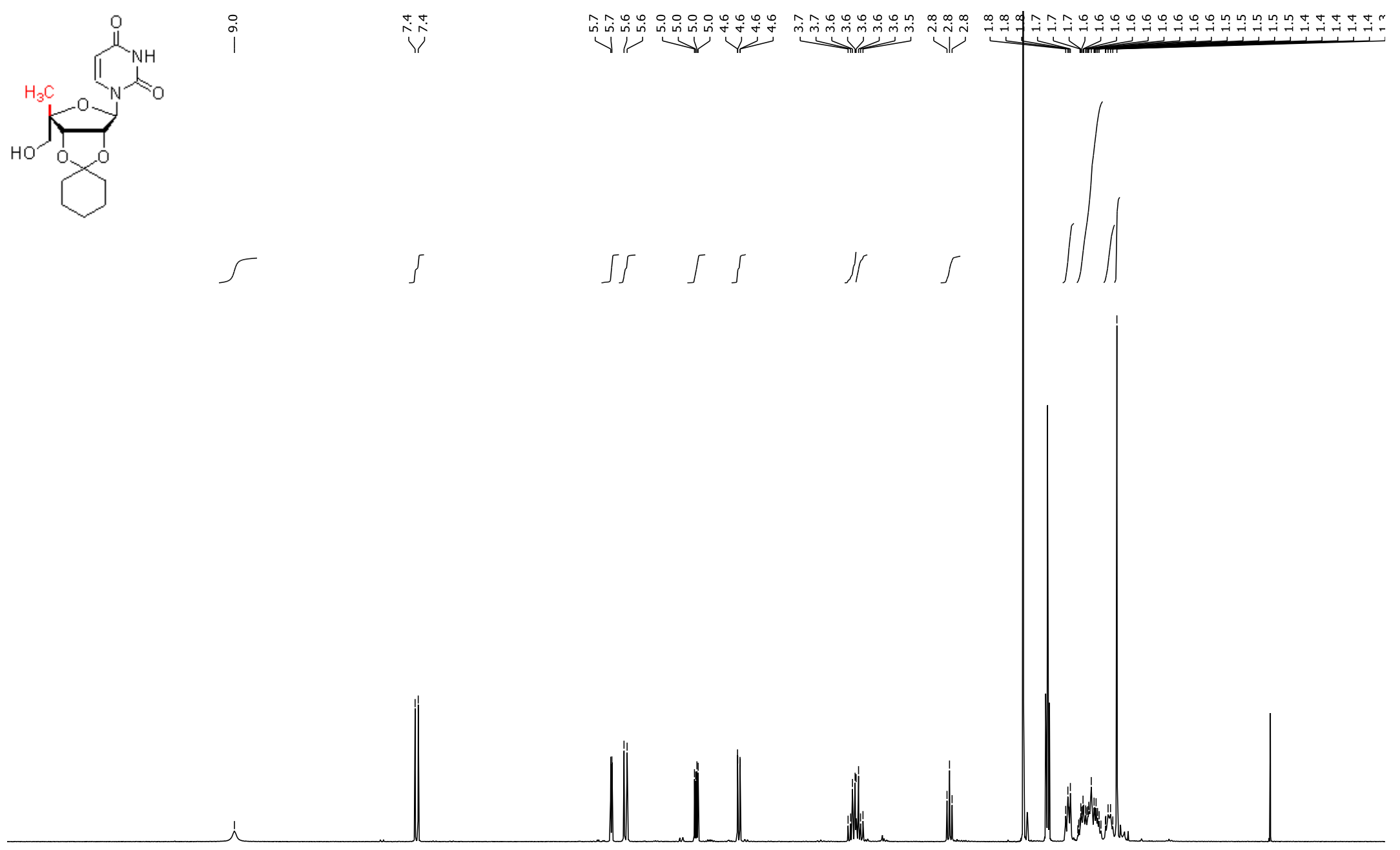

$1.0 \quad 10.5 \quad 10.0$

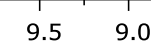

58.0

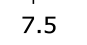

$6.5 \quad 6.0$

$5.5 \quad 5.0$

Figure S19. ${ }^{1} \mathrm{H}$ NMR $\left(300 \mathrm{MHz}, \mathrm{CD}_{3} \mathrm{CN}\right)$ spectrum of compound 6. 

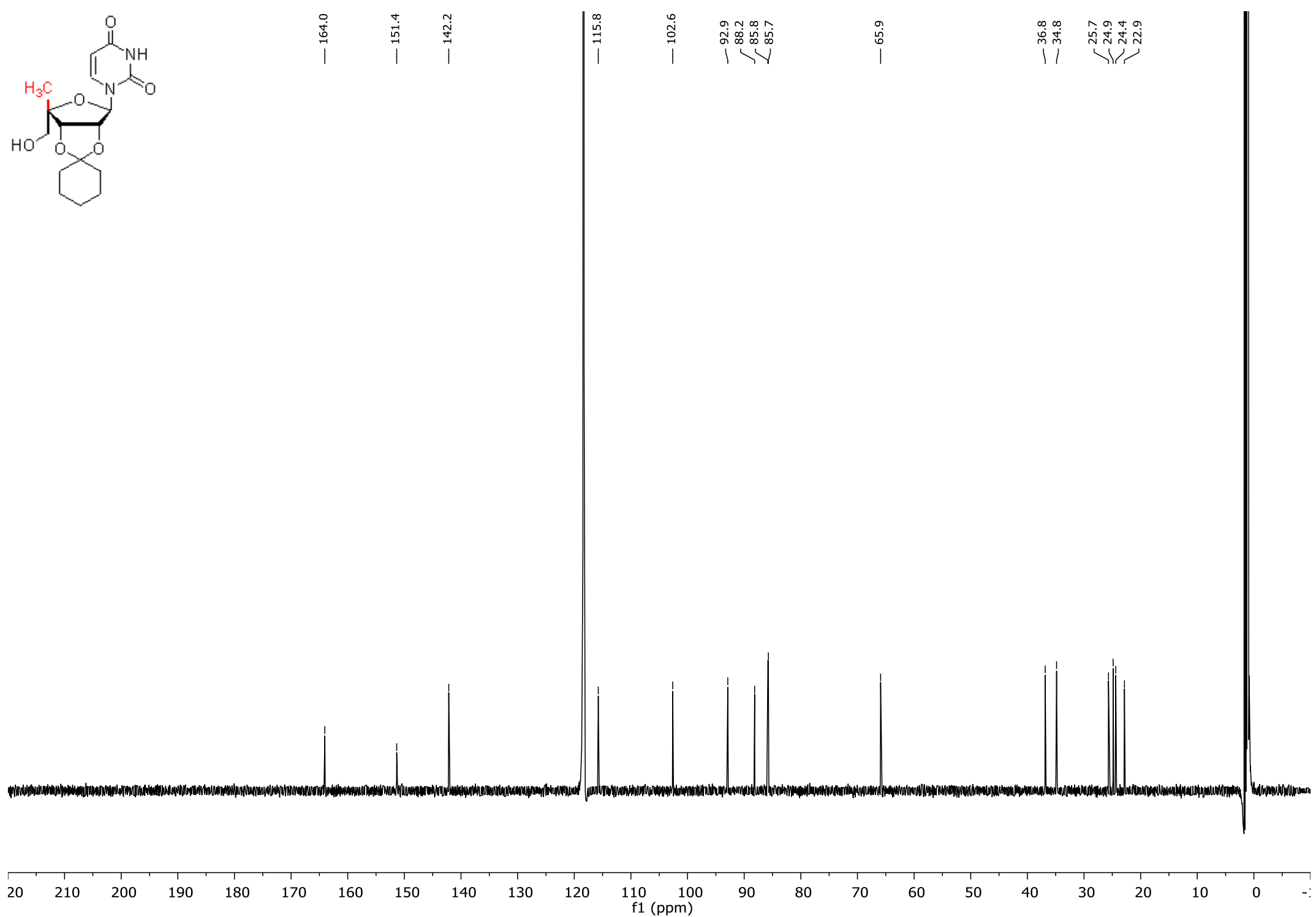

Figure S20. ${ }^{13} \mathrm{C}$ NMR (126 MHz, $\mathrm{CD}_{3} \mathrm{CN}$ ) spectrum of compound 6. 


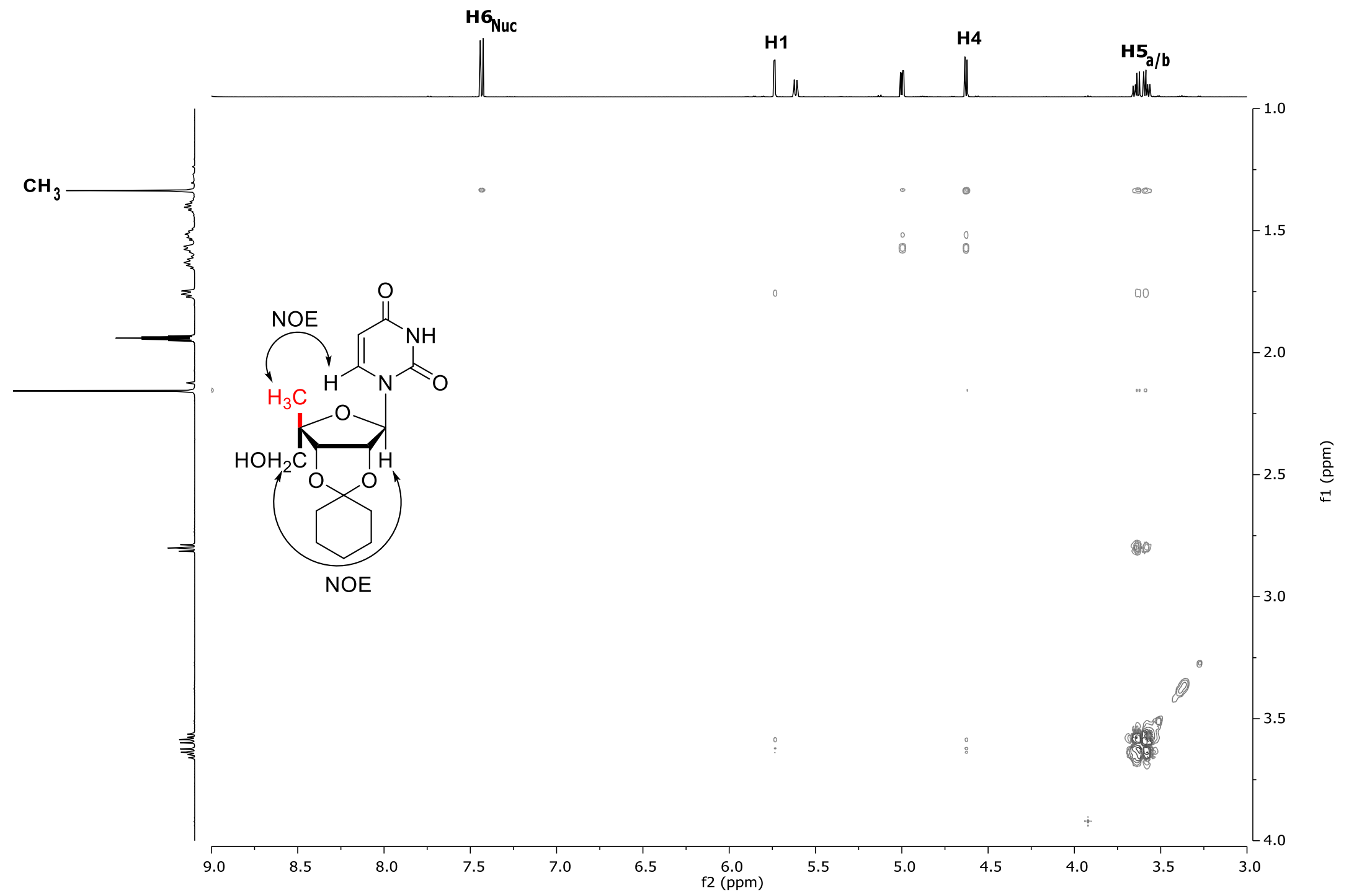

Figure S21. Partial NOESY ( $\left.400 \mathrm{MHz}, \mathrm{CD}_{3} \mathrm{CN}\right)$ of compound 6. 


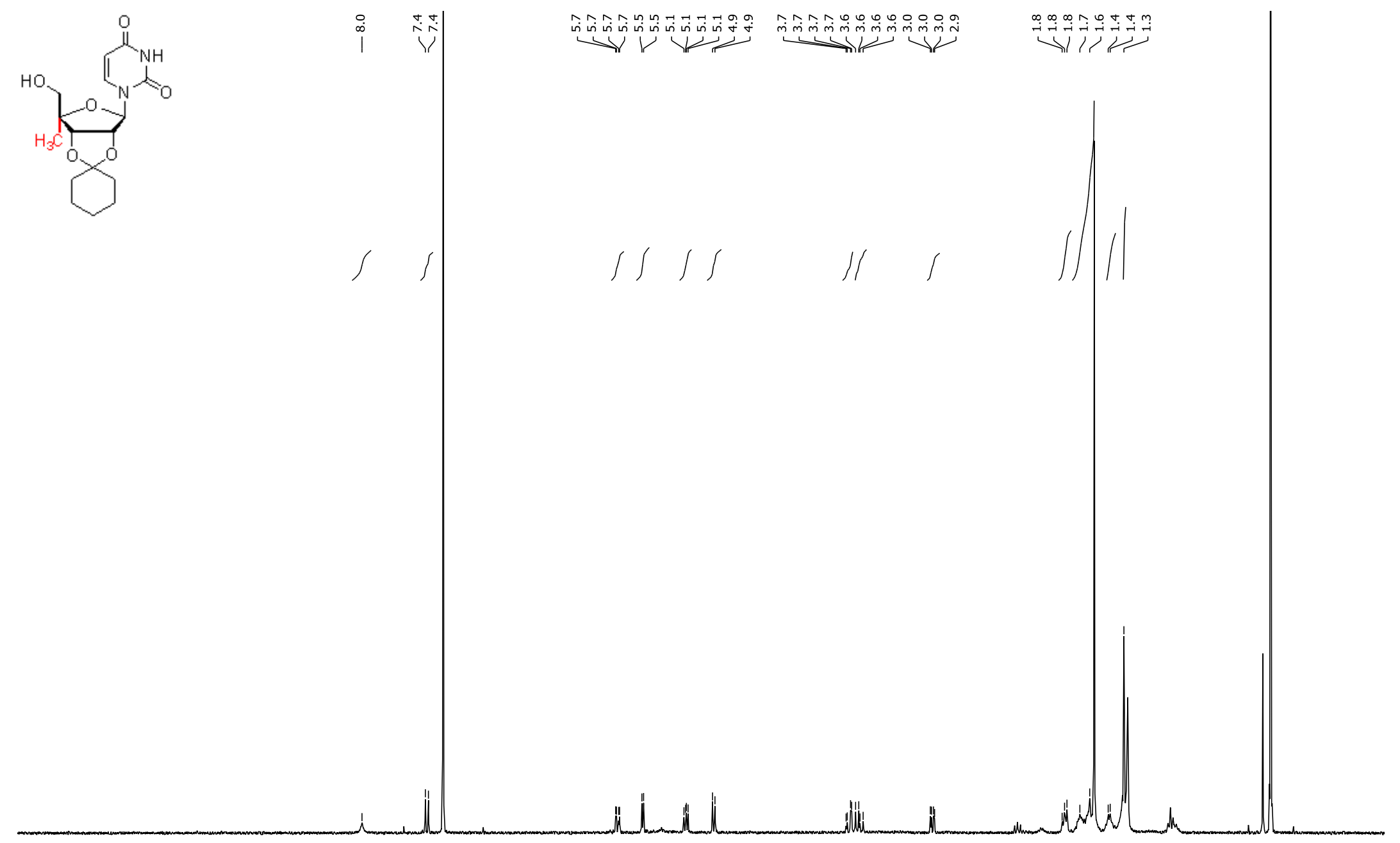

$\begin{array}{llllllllllllllllllllllll}10.5 & 10.0 & 9.5 & 9.0 & 8.5 & 8.0 & 7.5 & 7.0 & 6.5 & 6.0 & 5.5 & \begin{array}{c}5.0 \\ \text { 5 }\end{array} & 4.5 & 4.0 & 3.5 & 3.0 & 2.5 & 2.0 & 1.5 & 1.0 & 0.5 & 0.0 & -0.5 & -1\end{array}$

Figure S22. ${ }^{1} \mathrm{H} \mathrm{NMR}\left(300 \mathrm{MHz}, \mathrm{CDCl}_{3}\right)$ spectrum of compound $\mathbf{6 a}$. 


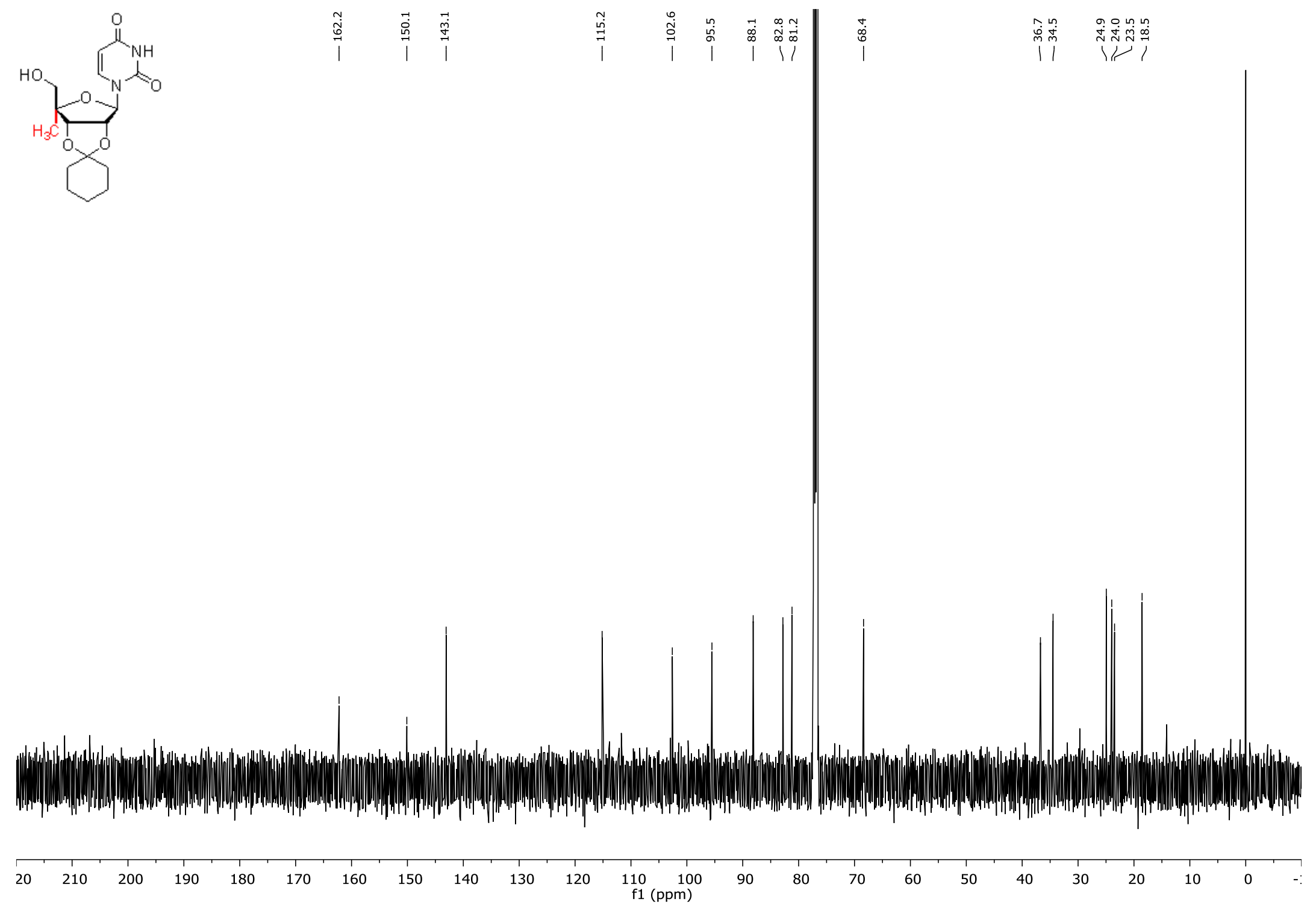

Figure S23. ${ }^{13} \mathrm{C}$ NMR $\left(126 \mathrm{MHz}, \mathrm{CDCl}_{3}\right)$ spectrum of compound $\mathbf{6 a}$. 

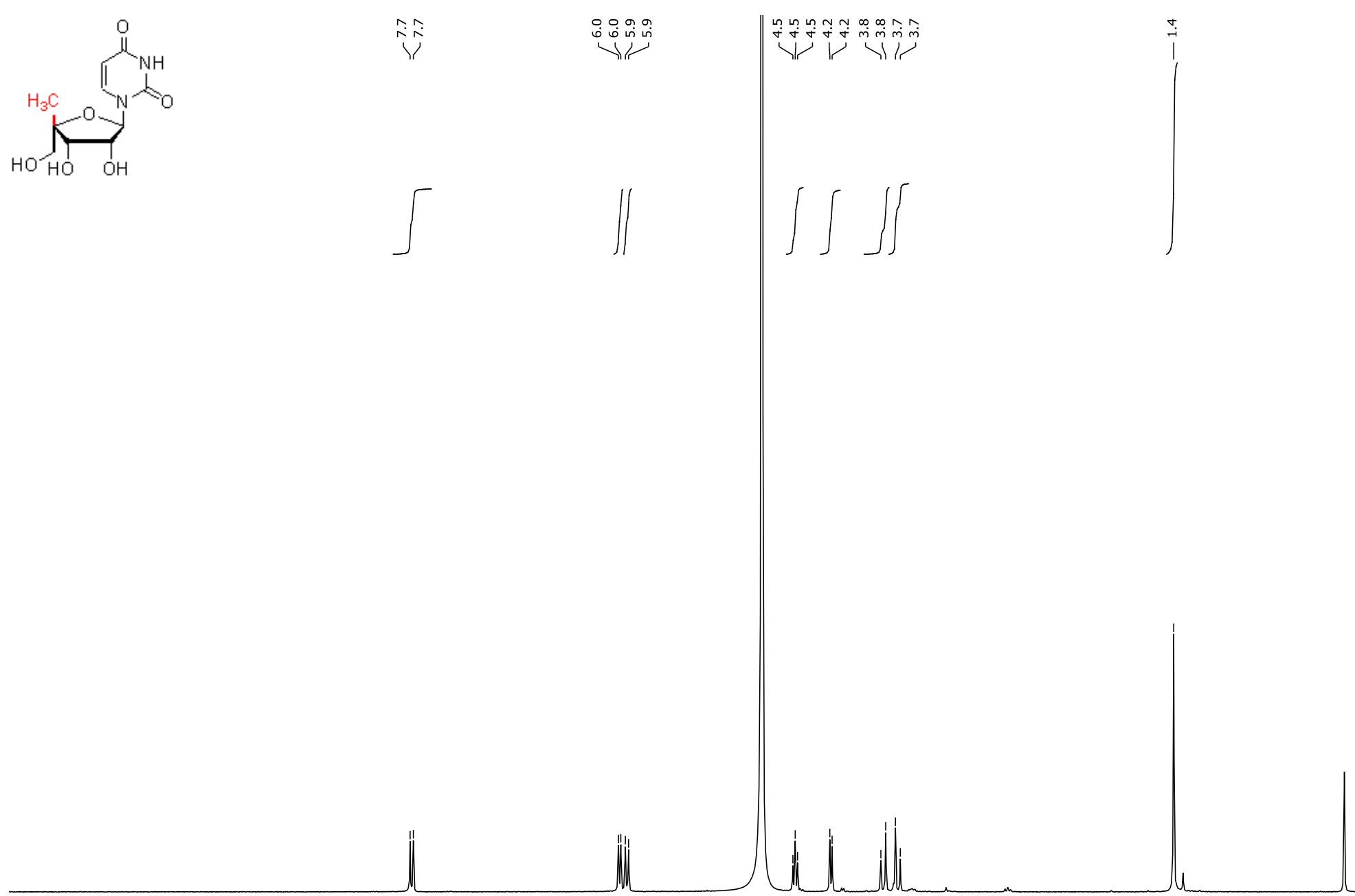

$1.0 \quad 10.5 \quad 10.0$ $9.0 \quad 8.5-8.0$ $\begin{array}{llll}1 & & & \\ 7.5 & 7.0 & 6.5\end{array}$ $\begin{array}{lll}6.0 & 5.5 & 5.0 \\ \mathrm{f} 1(\mathrm{ppm})\end{array}$

Figure S24. ${ }^{1} \mathrm{H}$ NMR (300 MHz, $\left.\mathrm{D}_{2} \mathrm{O}\right)$ spectrum of compound 7. 


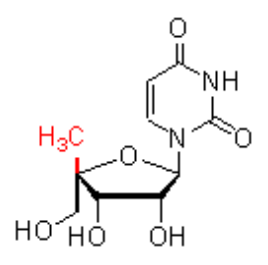

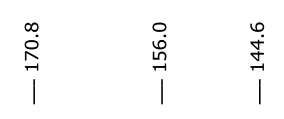

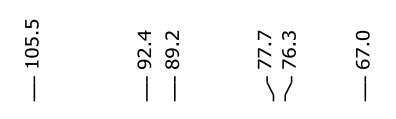

$\stackrel{\circ}{\stackrel{+}{i}}$

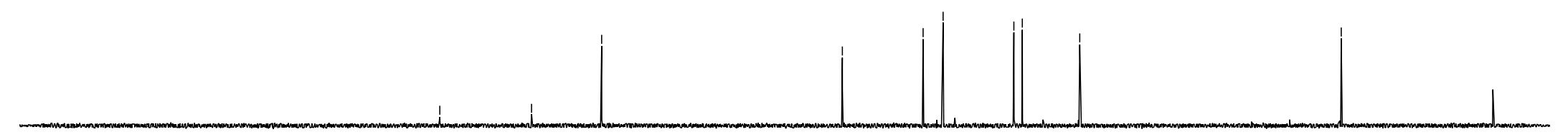

$\begin{array}{llllllllllllllllllllllllllll}230 & 220 & 210 & 200 & 190 & 180 & 170 & 160 & 150 & 140 & 130 & 120 & 110 & 100 & 90 & 80 & 70 & 60 & 50 & 40 & 30 & 20 & 10 & 0 & \end{array}$

Figure S25. ${ }^{13} \mathrm{C}$ NMR (126 MHz, $\left.\mathrm{D}_{2} \mathrm{O}\right)$ spectrum of compound 7. 

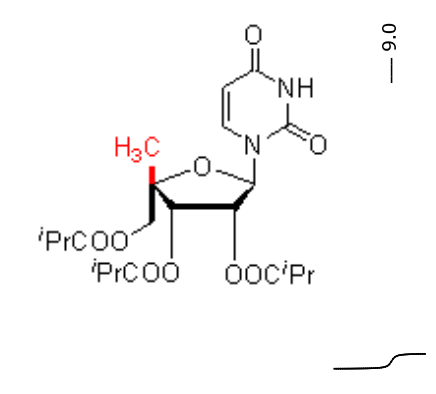

Figure S26. ${ }^{1} \mathrm{H}$ NMR $\left(300 \mathrm{MHz}, \mathrm{CD}_{3} \mathrm{CN}\right)$ spectrum of compound 8. 


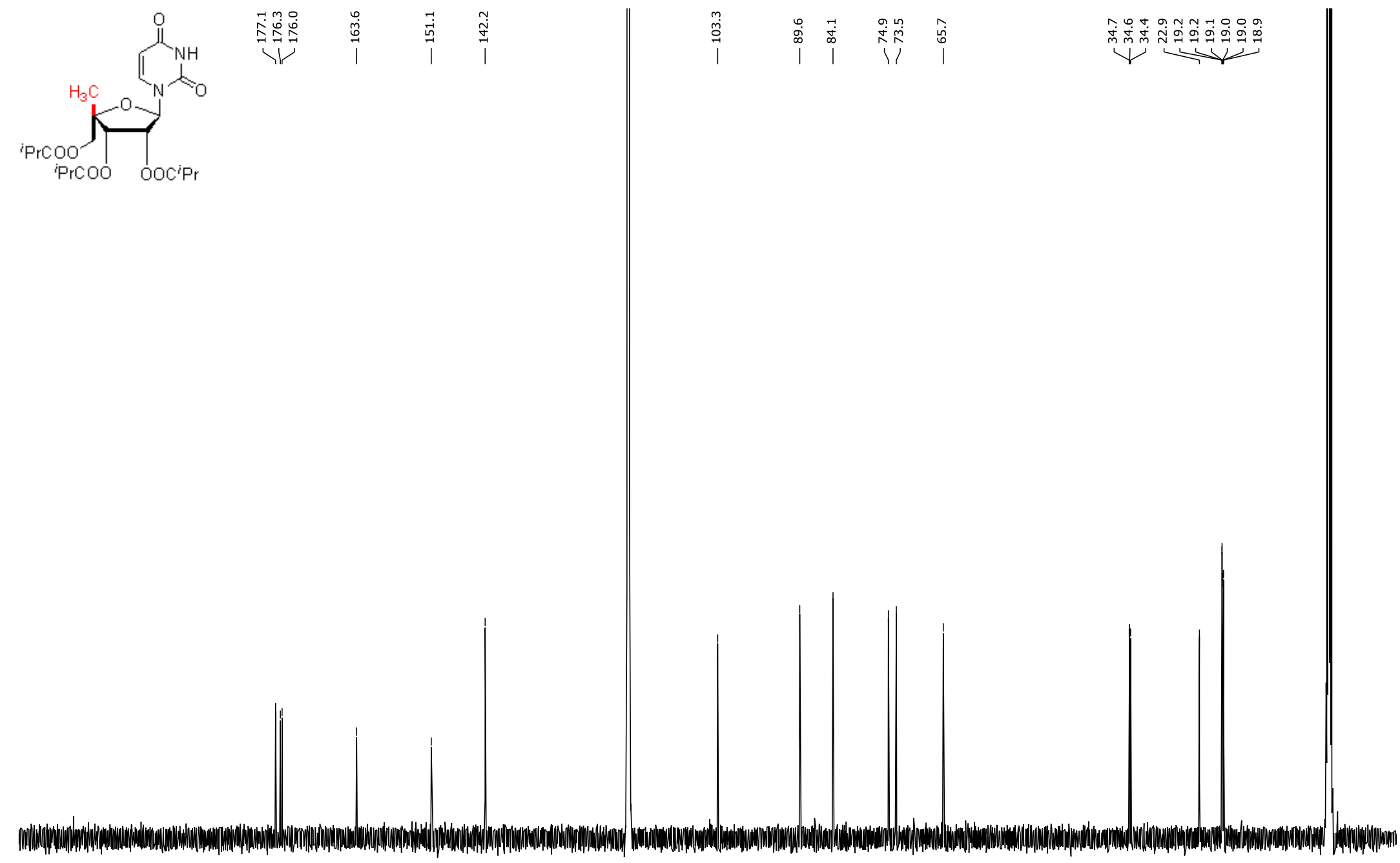

20

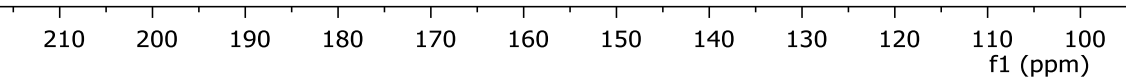

Figure S27. ${ }^{13} \mathrm{C}$ NMR $\left(126 \mathrm{MHz}, \mathrm{CD}_{3} \mathrm{CN}\right)$ spectrum of compound 8. 


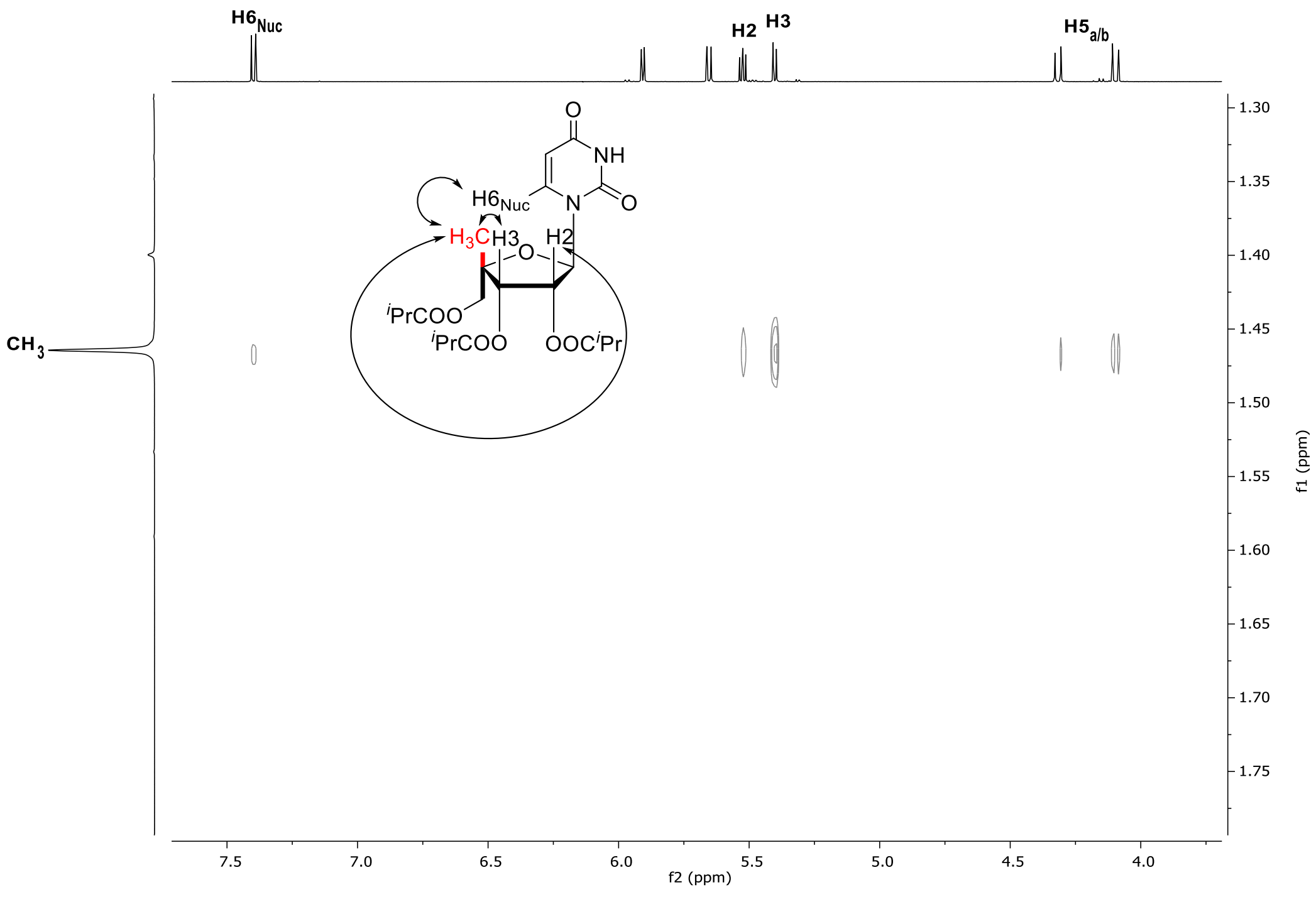

Figure S28. Partial NOESY ( $\left.500 \mathrm{MHz}, \mathrm{CD}_{3} \mathrm{CN}\right)$ of compound 8. 

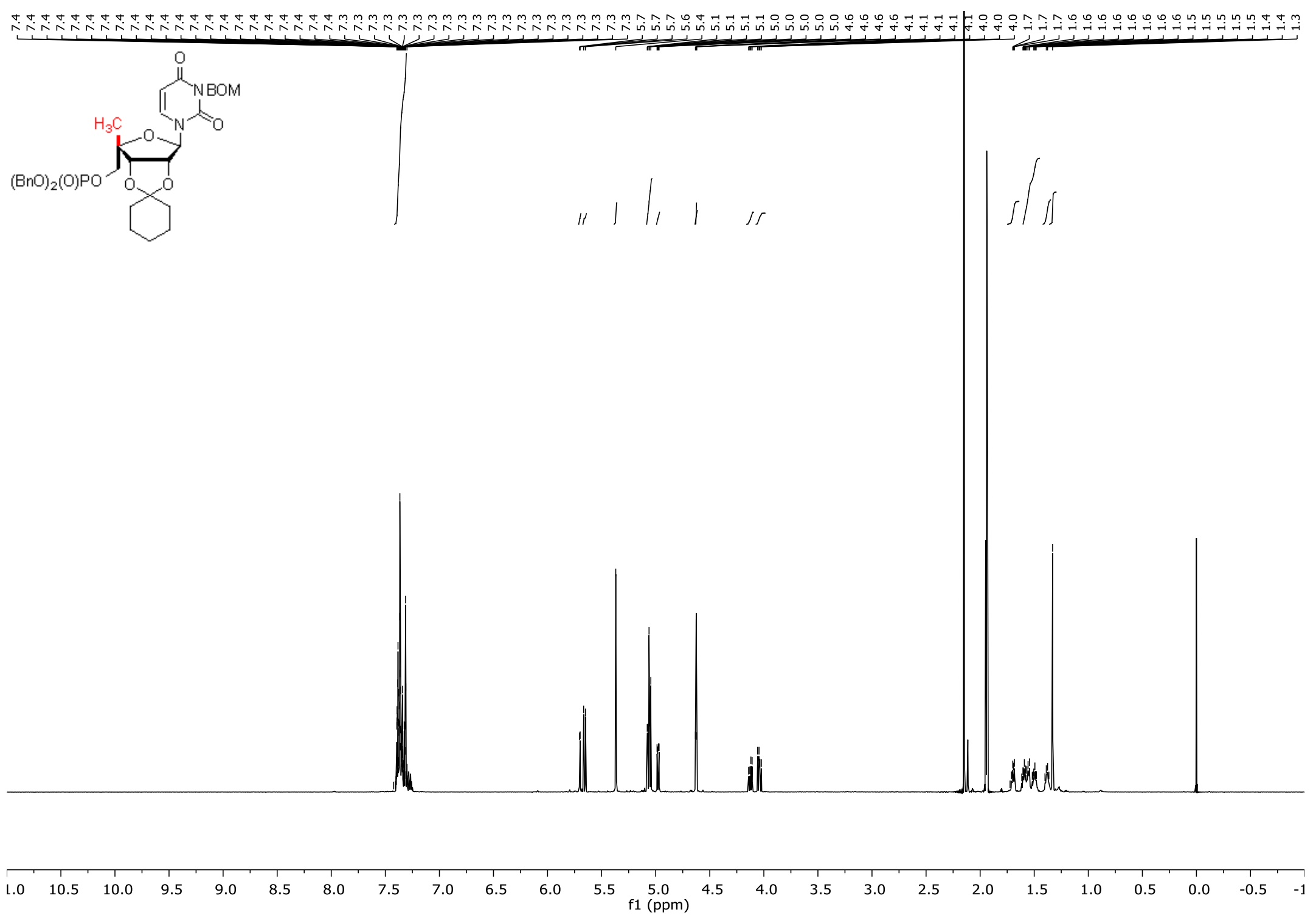

Figure S29. ${ }^{1} \mathrm{H} \mathrm{NMR}\left(500 \mathrm{MHz}, \mathrm{CD}_{3} \mathrm{CN}\right)$ spectrum of compound 9. 


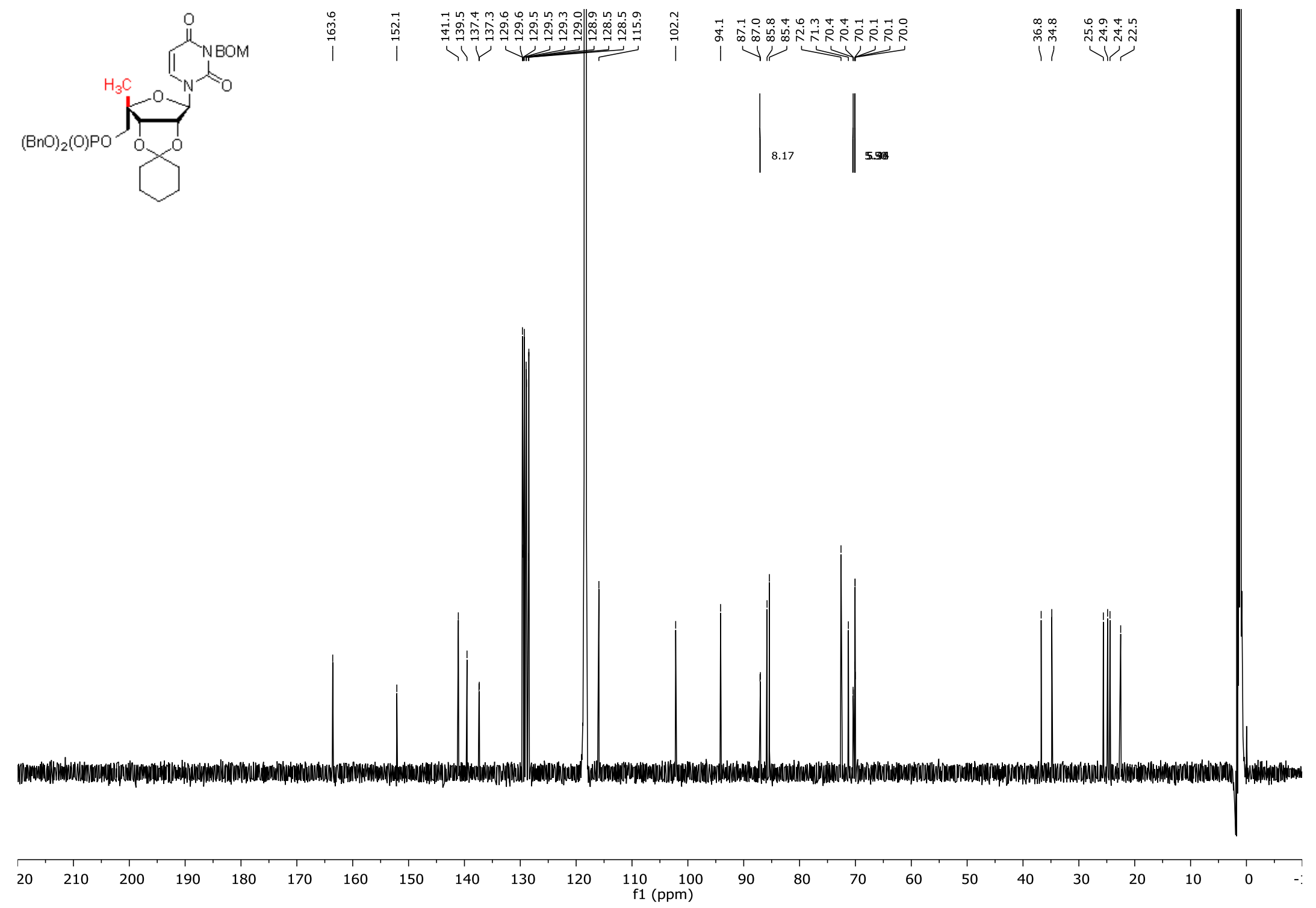

Figure S30. ${ }^{13} \mathrm{C}$ NMR (126 MHz, $\mathrm{CD}_{3} \mathrm{CN}$ ) spectrum of compound 9. 

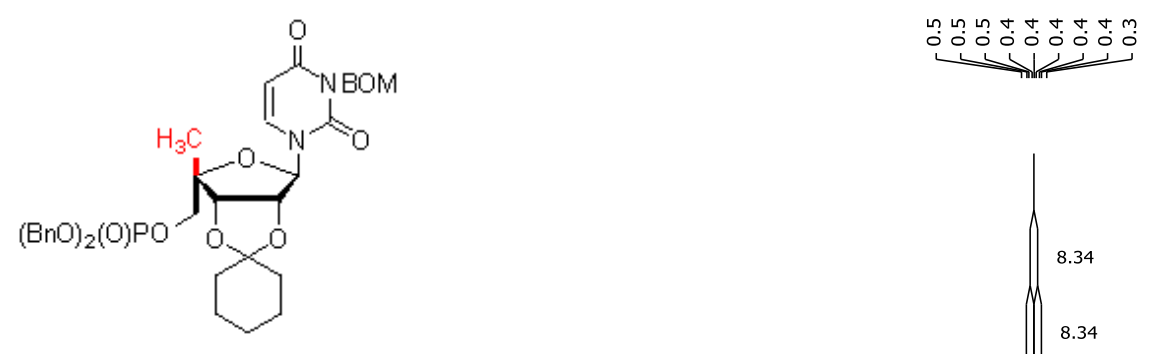

M.34 $6 \begin{aligned} & 8.34 \\ & 6.06 \\ & 6.06\end{aligned}$

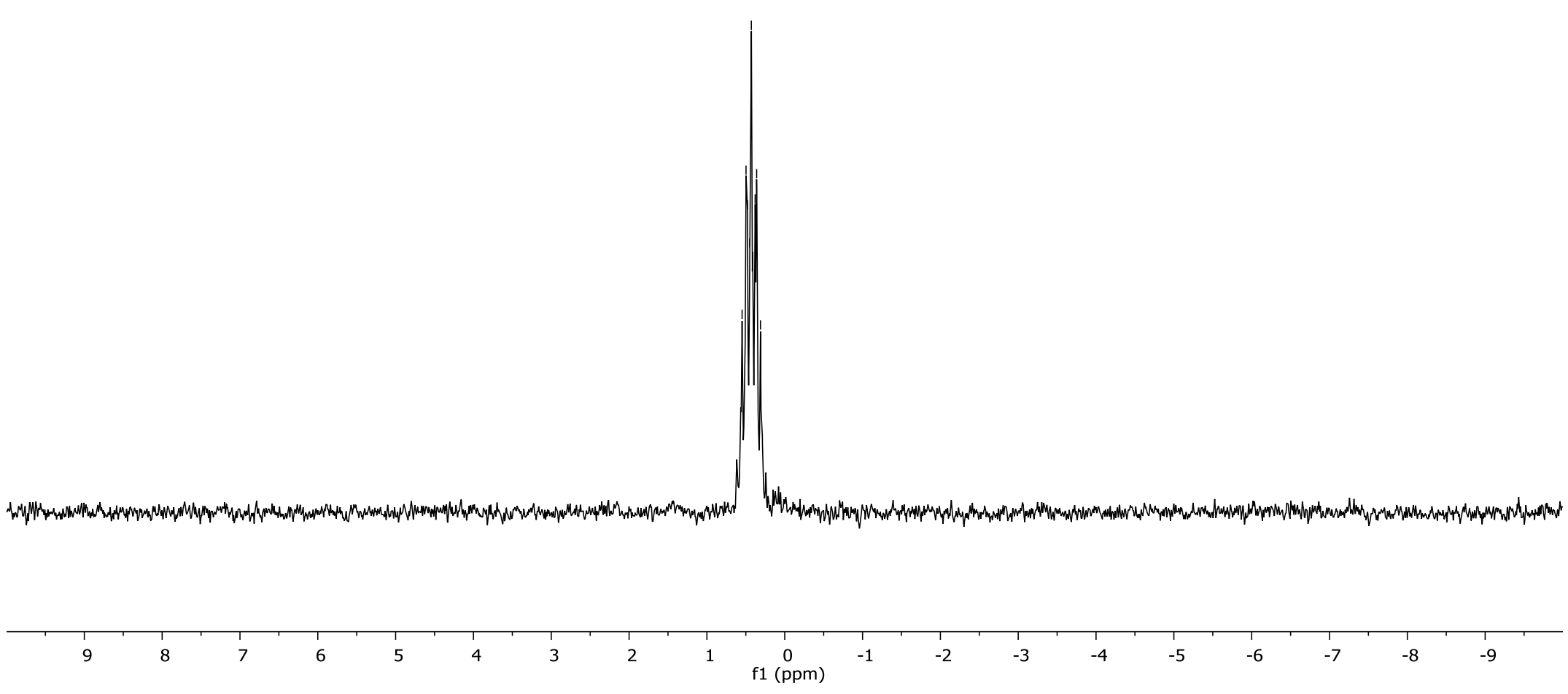

Figure S31. ${ }^{31} \mathrm{P}$ NMR (122 MHz, $\mathrm{CD}_{3} \mathrm{CN}$ ) spectrum of compound 9 (no ${ }^{1} \mathrm{H}$ decoupling). 

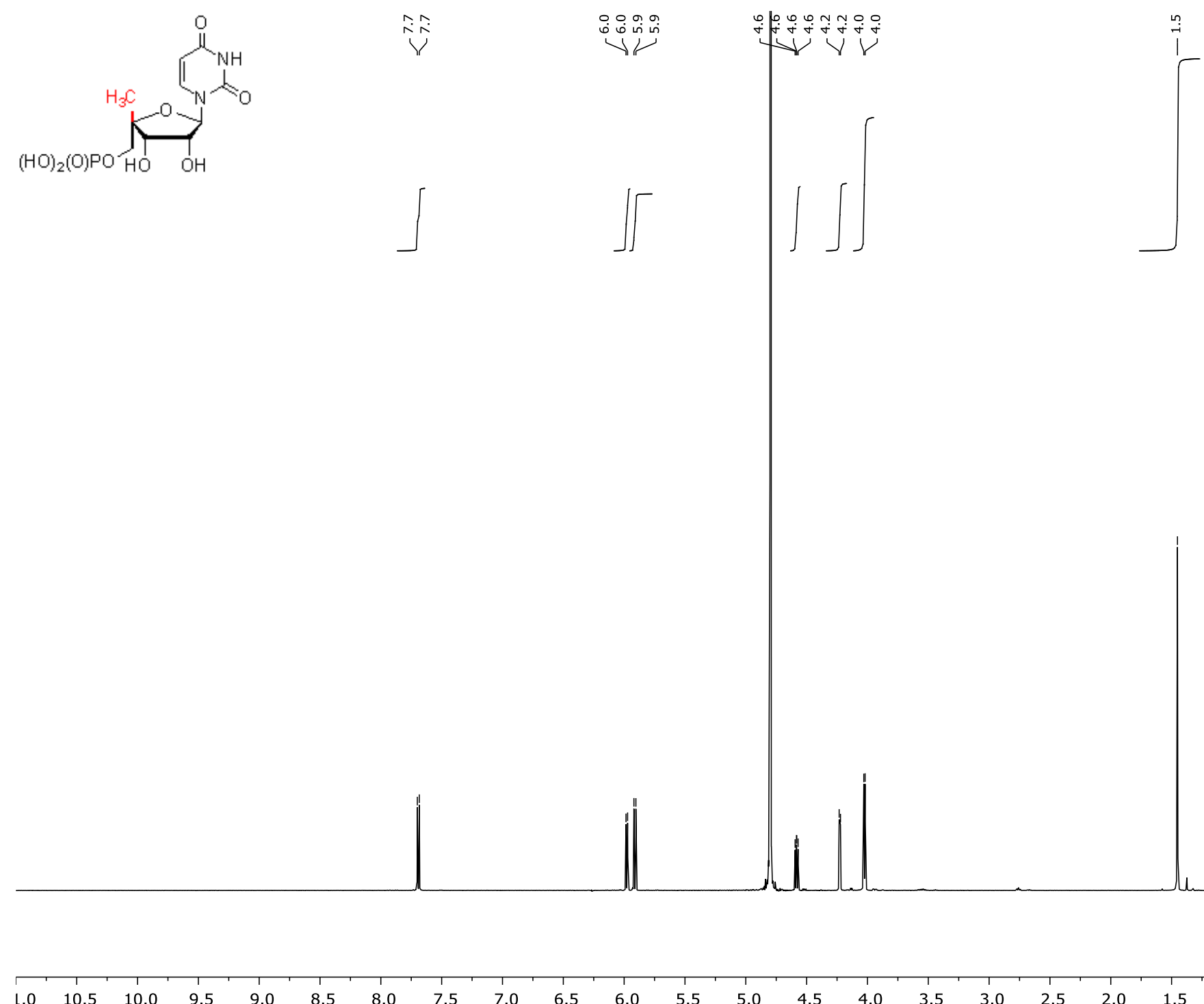

\begin{tabular}{l}
0.5 \\
\hline
\end{tabular}
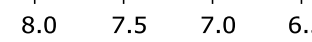

6.0

$5 \stackrel{5.0}{\mathrm{f} 1(\mathrm{ppm})}$

Figure S32. ${ }^{1} \mathrm{H}$ NMR $\left(500 \mathrm{MHz}, \mathrm{D}_{2} \mathrm{O}\right)$ spectrum of compound 10. 

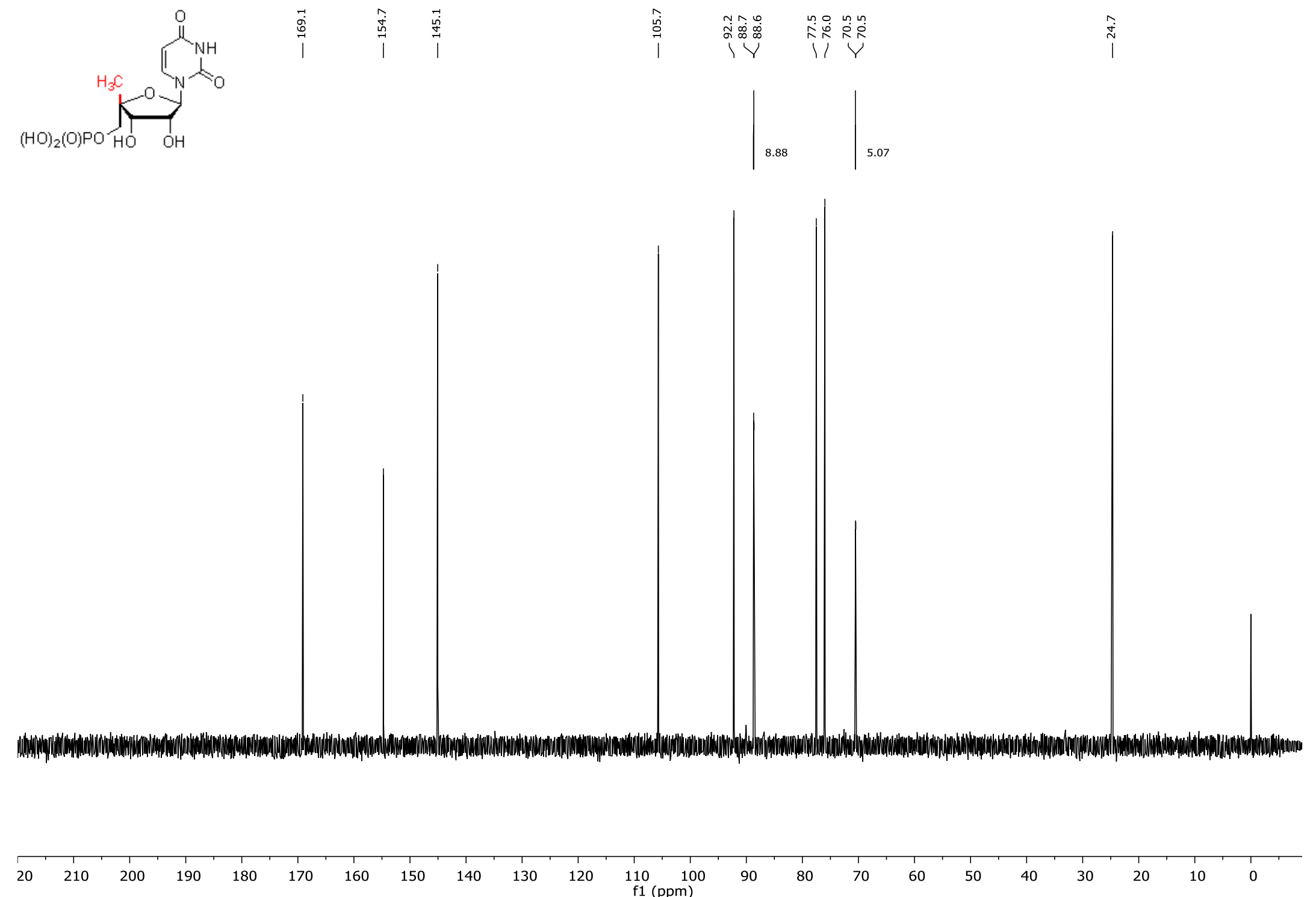

Figure S33. ${ }^{13} \mathrm{C}$ NMR (126 MHz, $\mathrm{D}_{2} \mathrm{O}$ ) spectrum of compound 10. 


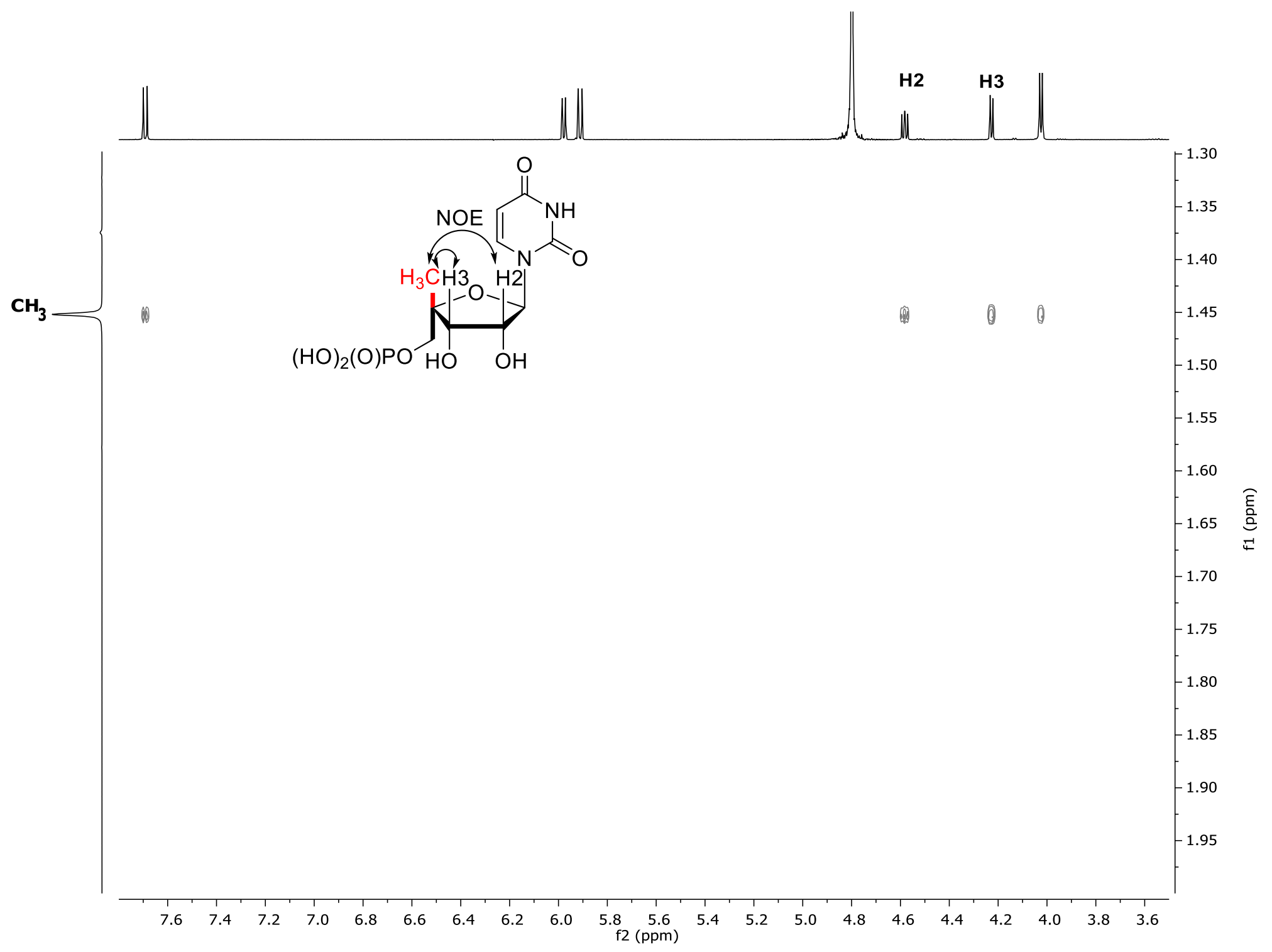

Figure S34. Partial NOESY (400 MHz, $\left.\mathrm{D}_{2} \mathrm{O}\right)$ of compound 10. 

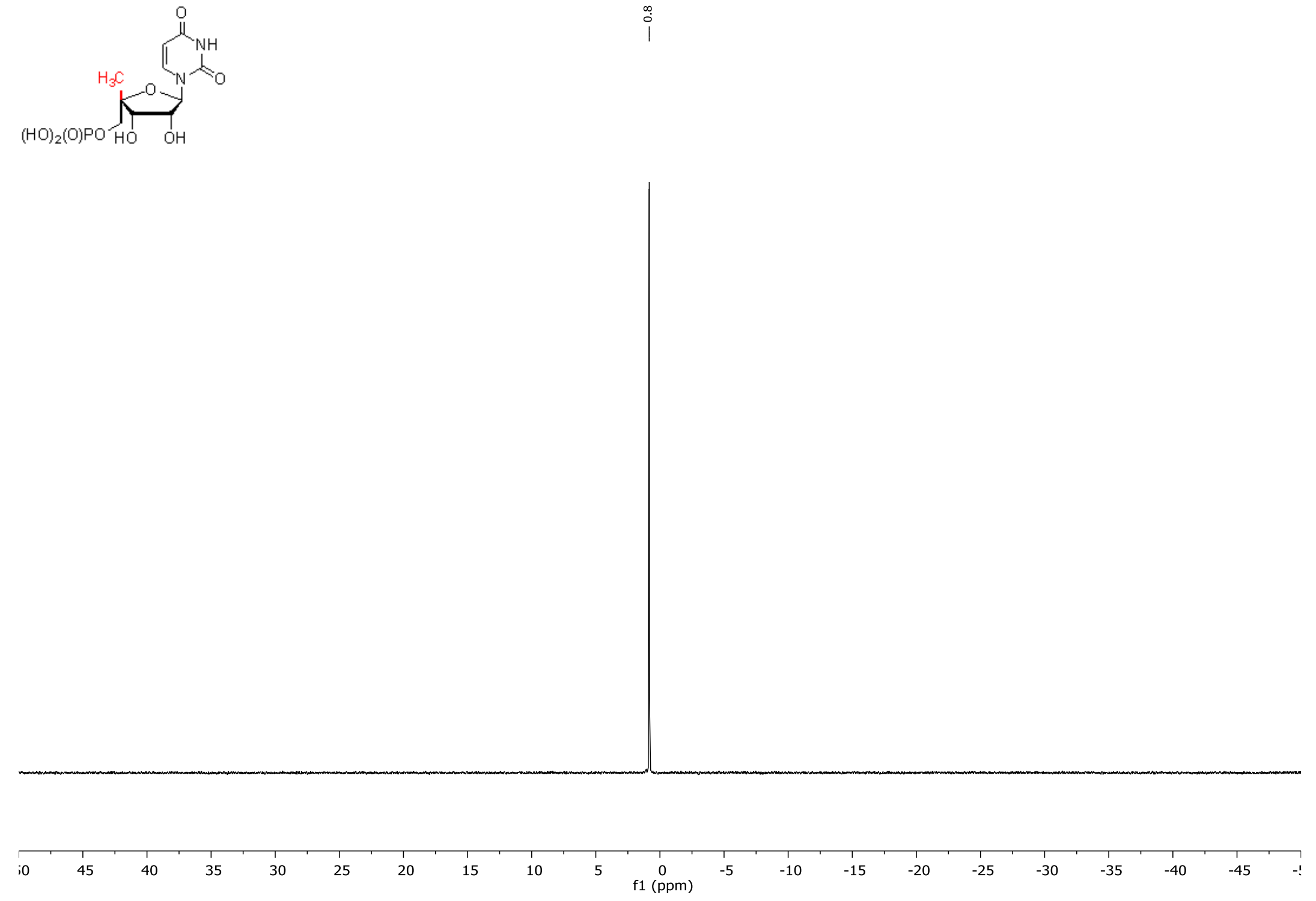

Figure S35. ${ }^{13} \mathrm{C}$ NMR (126 MHz, $\left.\mathrm{D}_{2} \mathrm{O}\right)$ spectrum of compound 10. 
L-Lyxose Spirocyclopropane Series 

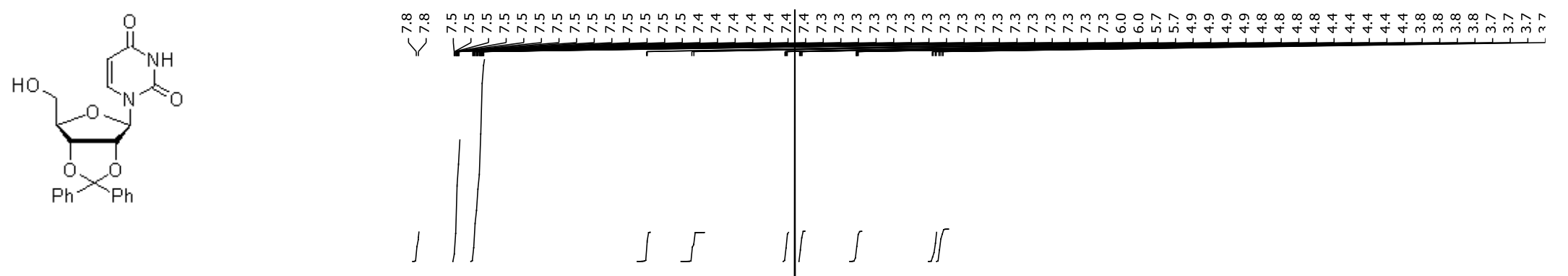

$\begin{array}{lll}\text { L. } & 10.5 & 10.0\end{array}$

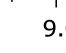

8.5
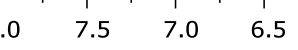

$\begin{array}{lll}6.0 & 5.5 \quad 5.0\end{array}$ f1 (ppm)

Figure S36. ${ }^{1} \mathrm{H}$ NMR $\left(500 \mathrm{MHz}, \mathrm{CD}_{3} \mathrm{OD}\right)$ spectrum of compound $\mathbf{1 b}$. 


$$
\text { ليلب }
$$



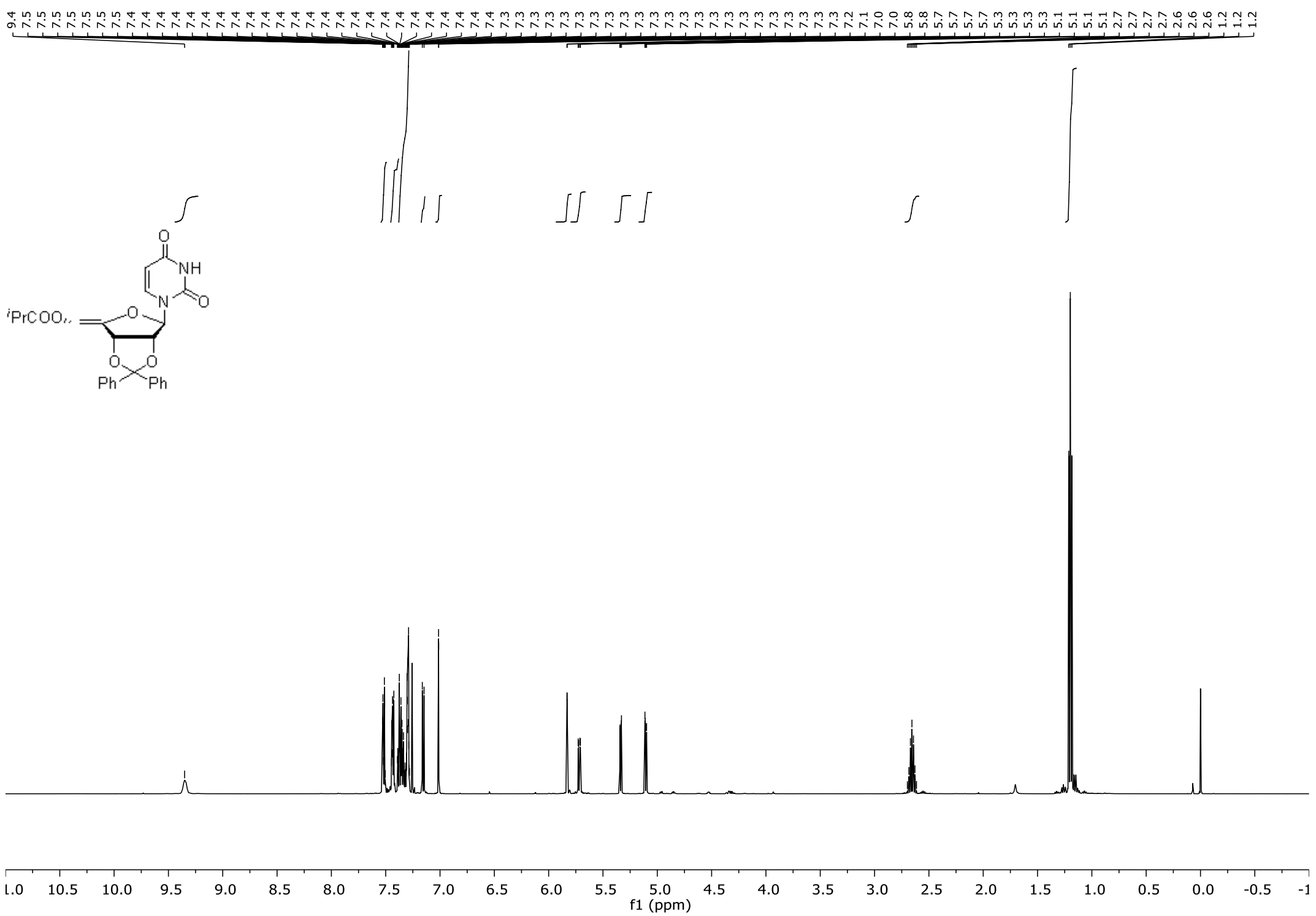

Figure S38. ${ }^{1} \mathrm{H}$ NMR $\left(500 \mathrm{MHz}, \mathrm{CDCl}_{3}\right)$ spectrum of compound $\mathbf{2 b}$. 

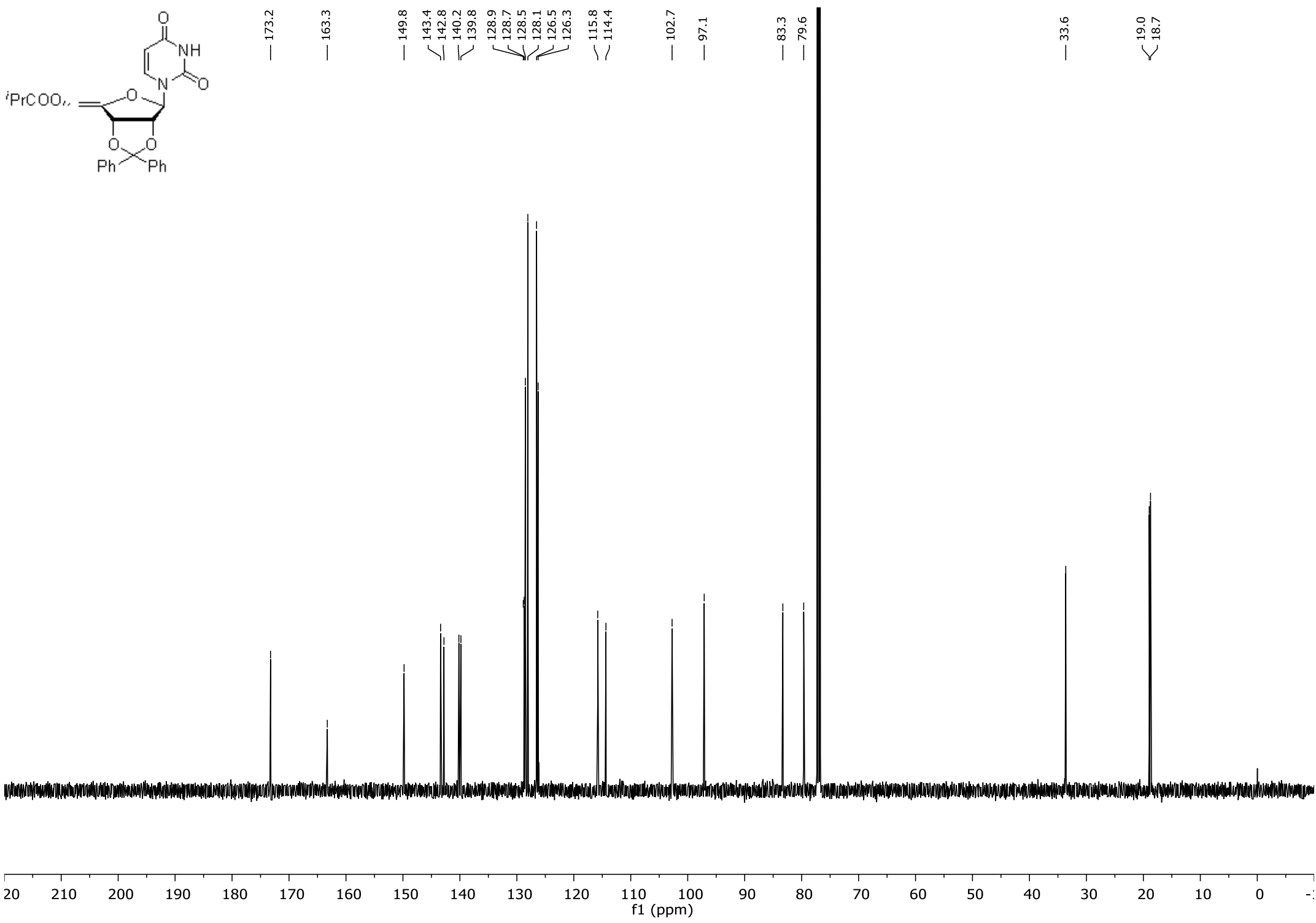

Figure S39. $\left.{ }^{13} \mathrm{C} \mathrm{NMR} \mathrm{(126} \mathrm{MHz,} \mathrm{CDCl}_{3}\right)$ spectrum of compound $\mathbf{2 b}$. 

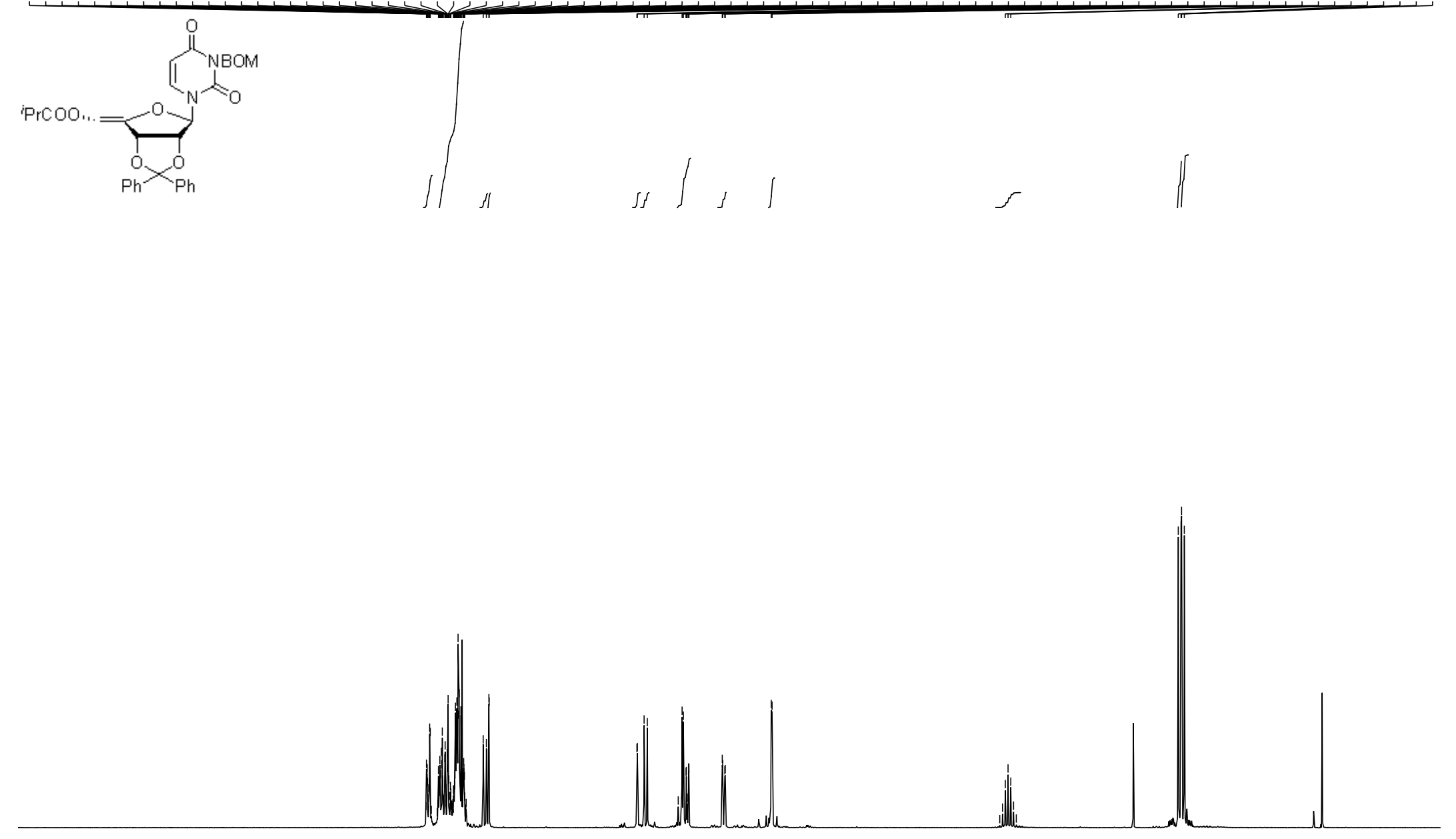

$10.5 \quad 10.0$

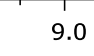

8.5
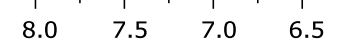

Figure S40. ${ }^{1} \mathrm{H}$ NMR (300 MHz, $\mathrm{CDCl}_{3}$ ) spectrum of compound $\mathbf{3 b}$. 

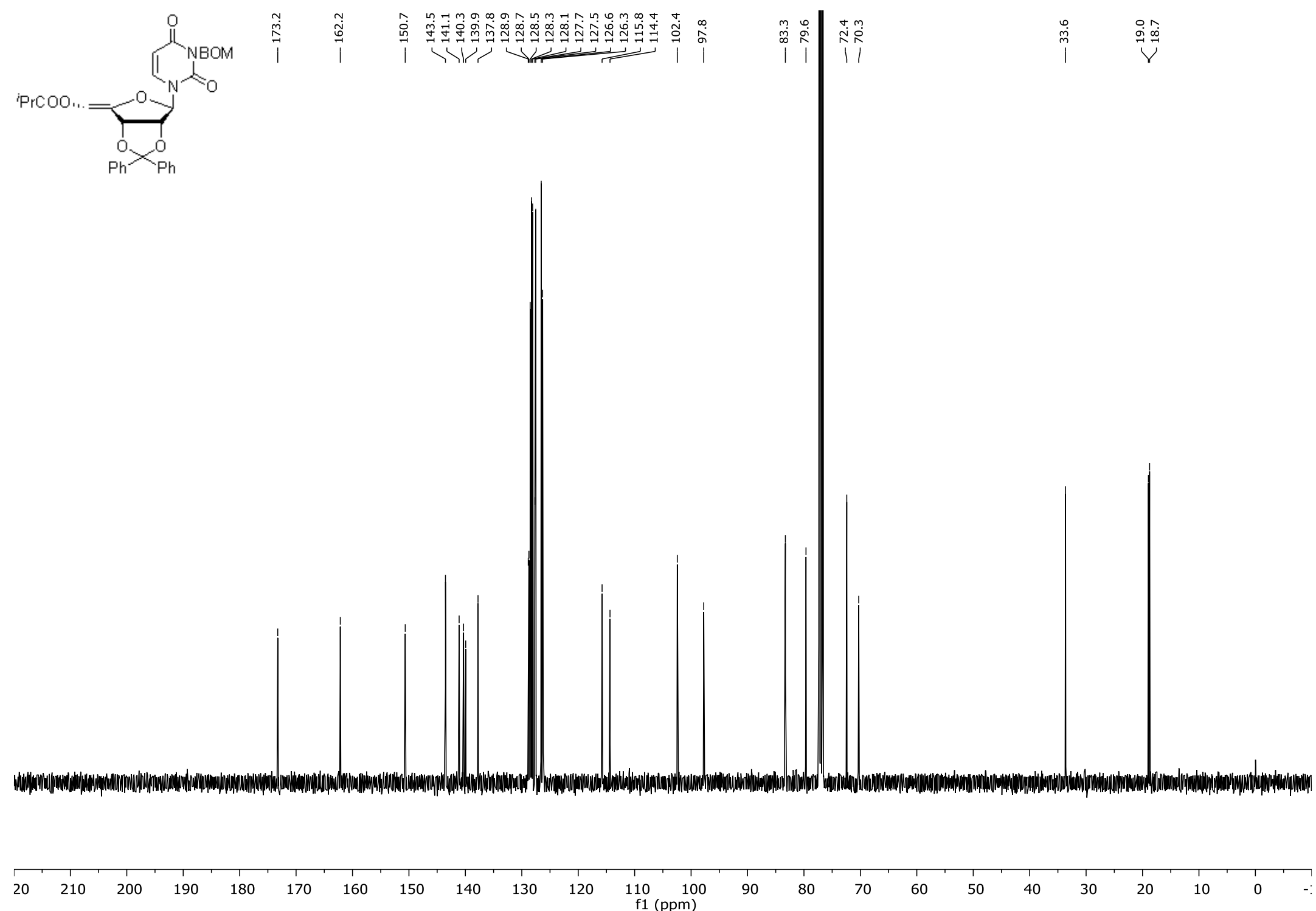

Figure S41. ${ }^{13} \mathrm{C} \mathrm{NMR}\left(101 \mathrm{MHz}, \mathrm{CDCl}_{3}\right)$ spectrum of compound $\mathbf{3 b}$. 

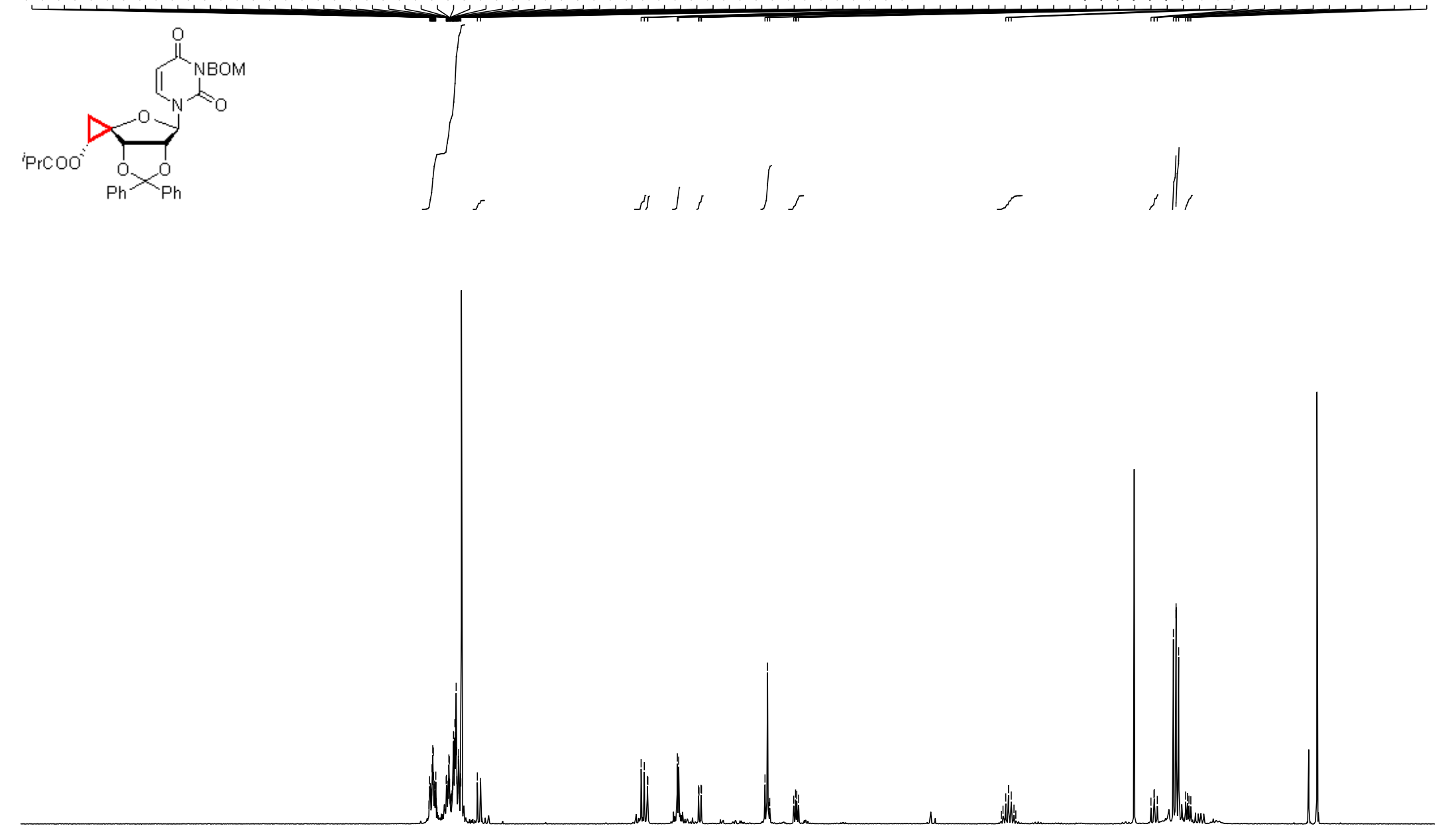

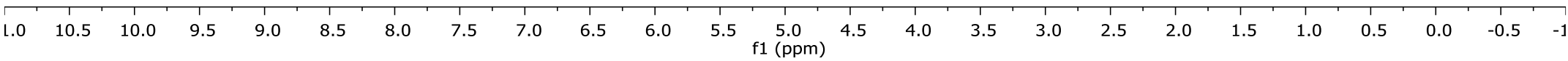

Figure S42. ${ }^{1} \mathrm{H}$ NMR $\left(300 \mathrm{MHz}, \mathrm{CDCl}_{3}\right)$ spectrum of compound $\mathbf{4 b}$. 


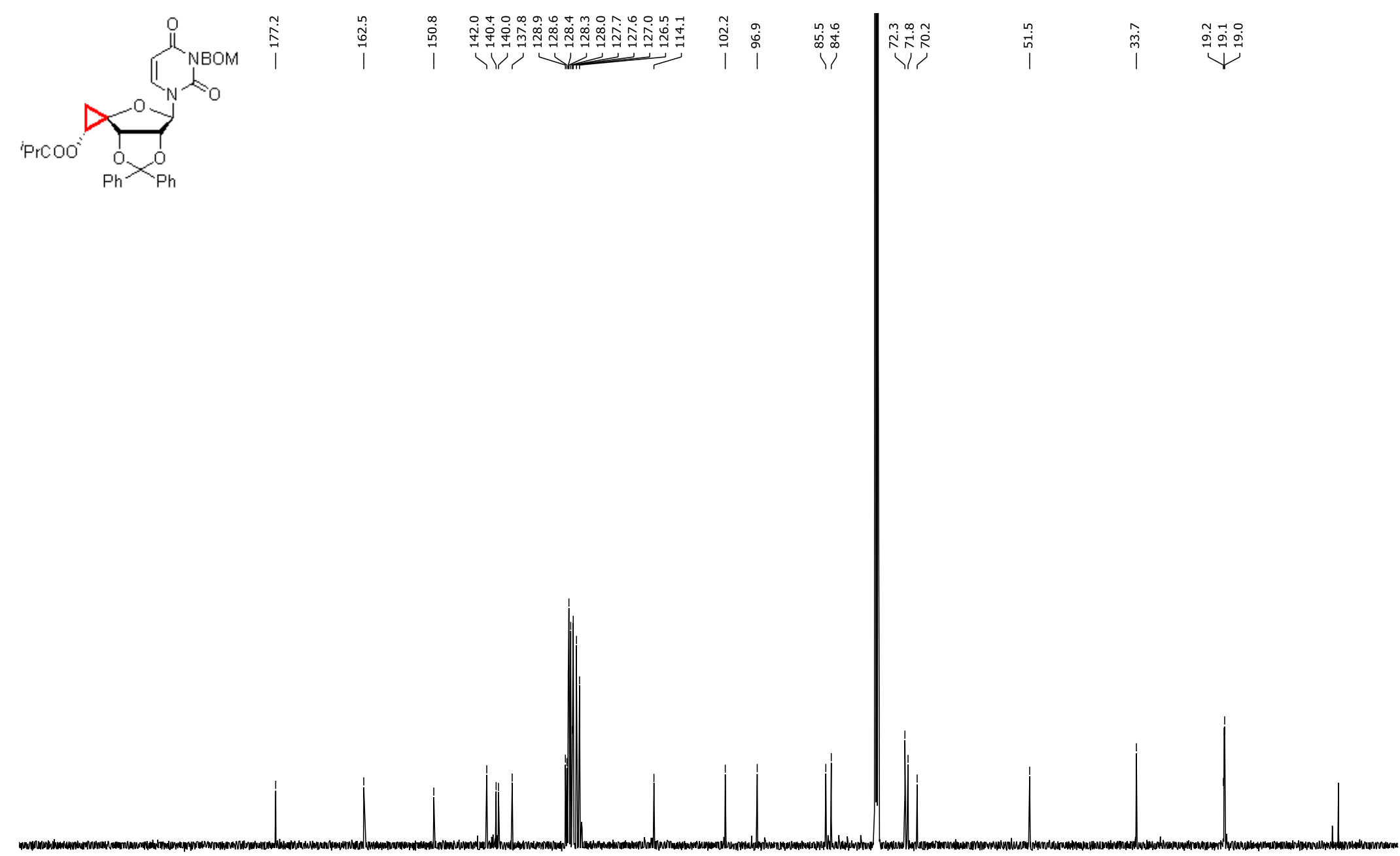

$210 \quad 200$

190

180

160

150

140

$130 \quad 12$ $110 \quad 100$
f1 (ppm)

Figure S43. ${ }^{13} \mathrm{C}$ NMR (126 MHz, $\mathrm{CDCl}_{3}$ ) spectrum of compound $\mathbf{4 b}$. 


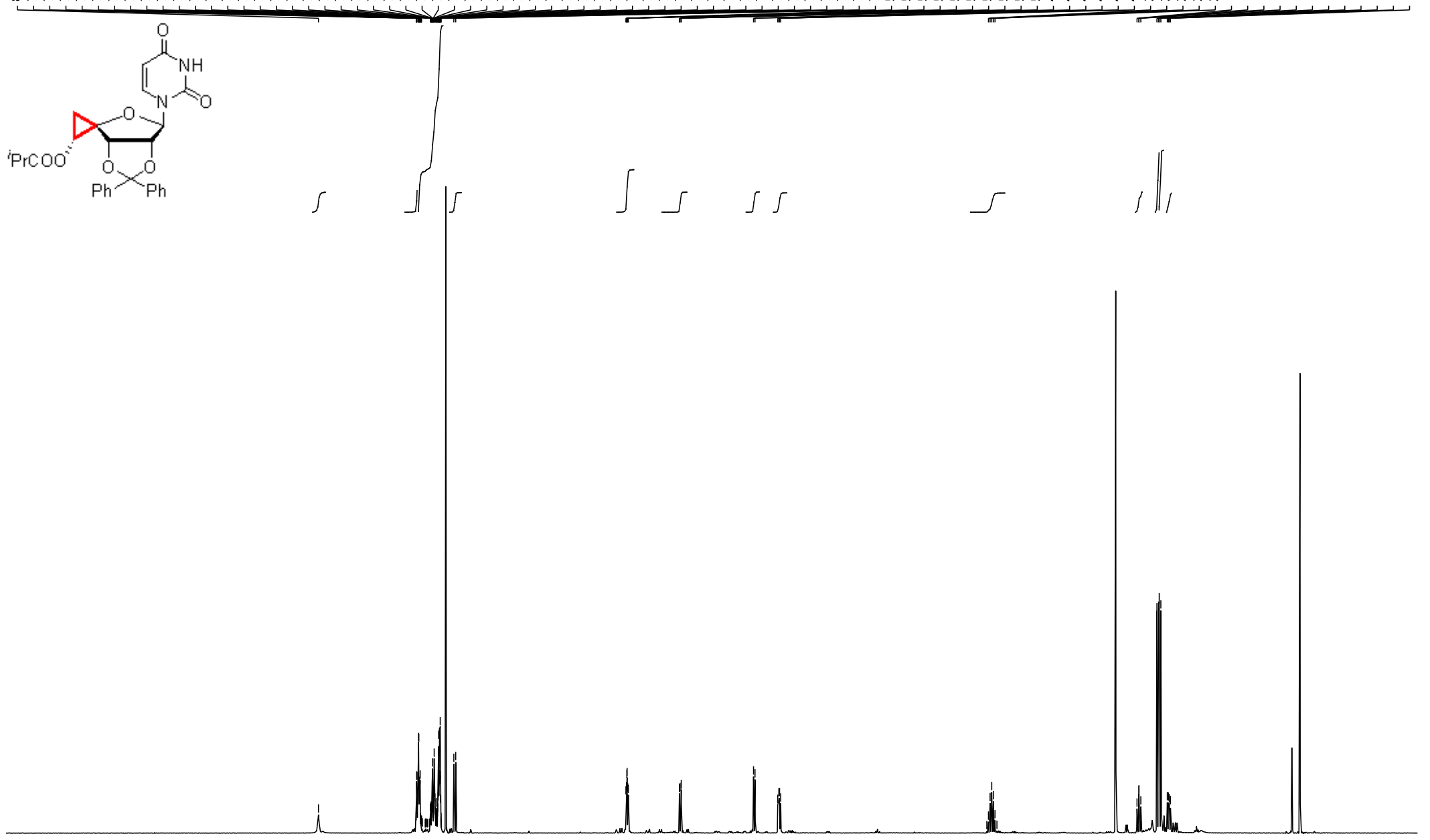

$\begin{array}{lllllll}10.5 & 10.0 & 9.5 & 9.0 & 8.5 & 8.0 & 7.5\end{array}$

Figure S44. ${ }^{1} \mathrm{H} \mathrm{NMR}\left(500 \mathrm{MHz}, \mathrm{CDCl}_{3}\right)$ spectrum of compound 11. 

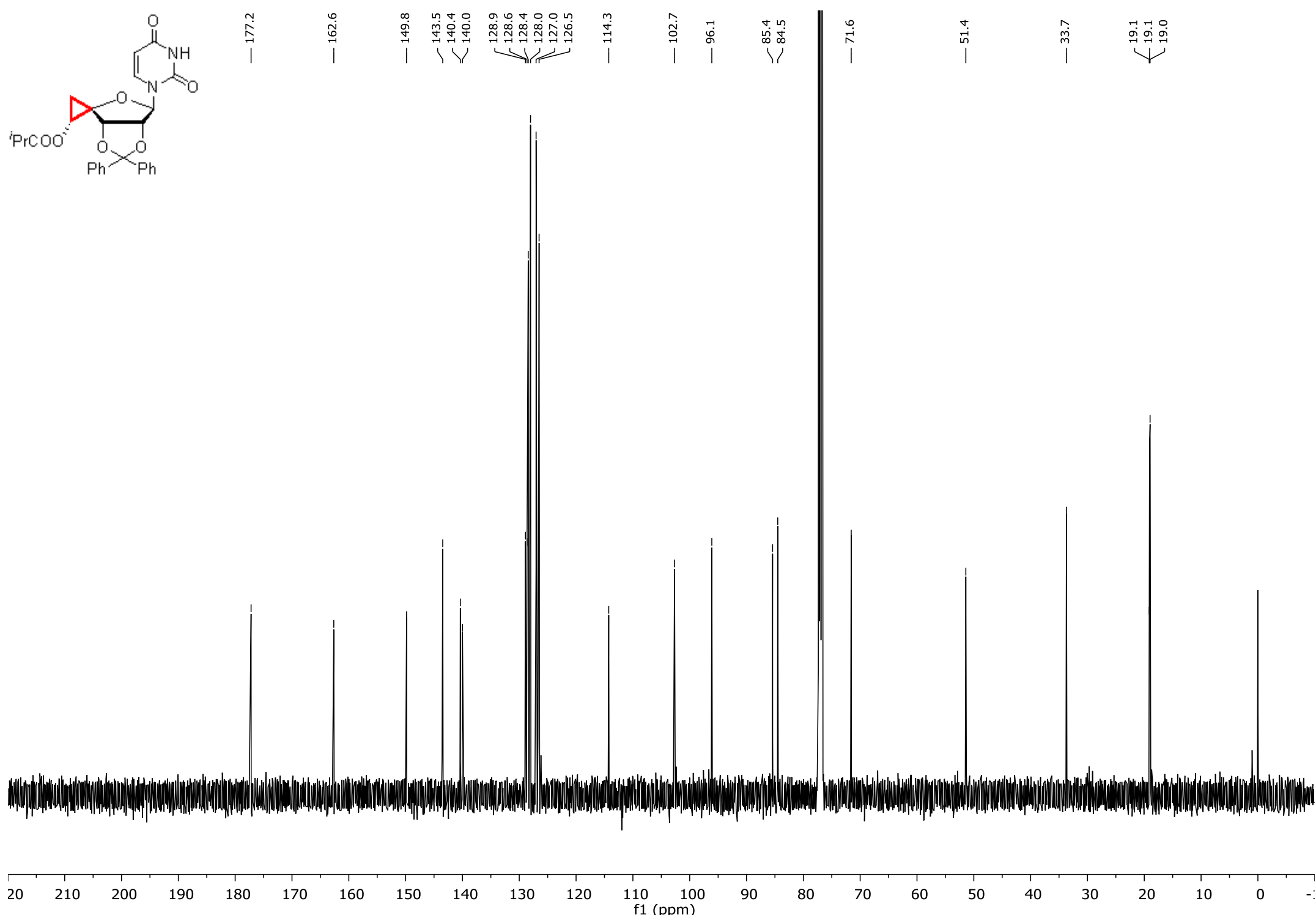

Figure S45. ${ }^{13} \mathrm{C} \mathrm{NMR}\left(126 \mathrm{MHz}, \mathrm{CDCl}_{3}\right)$ spectrum of compound 11. 

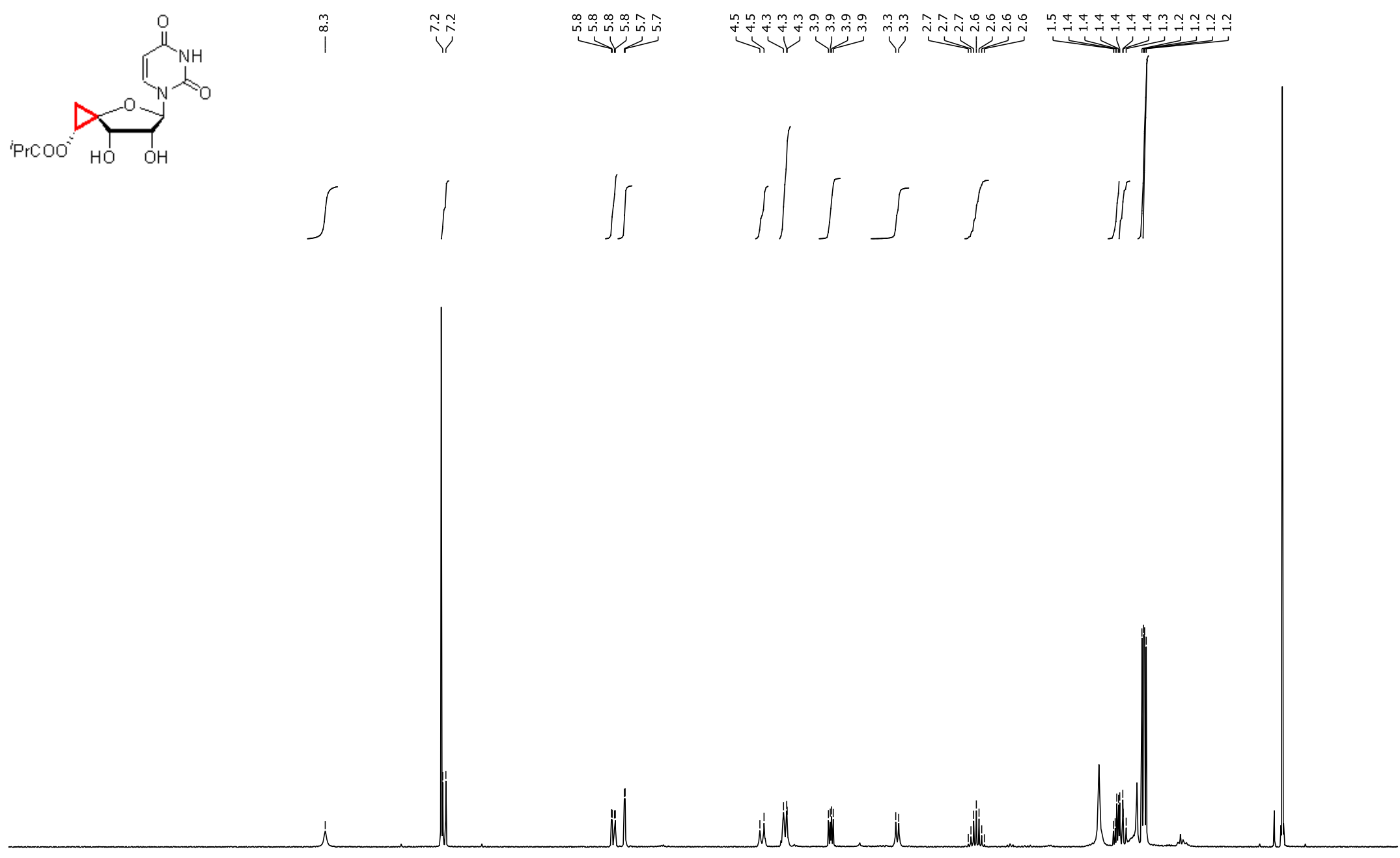

$10.5 \quad 10.0$

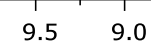

\begin{tabular}{l}
$0 \quad 5$ \\
\hline
\end{tabular}
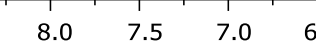

6.0

$5.5 \quad 5.0$

Figure S46. ${ }^{1} \mathrm{H}$ NMR (300 MHz, $\left.\mathrm{CDCl}_{3}\right)$ spectrum of compound 12. 

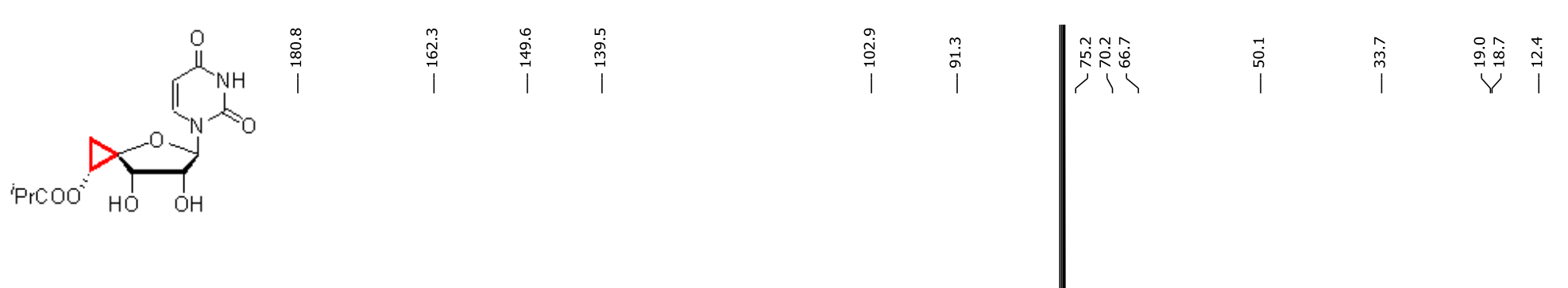

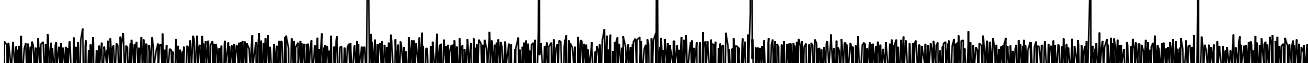
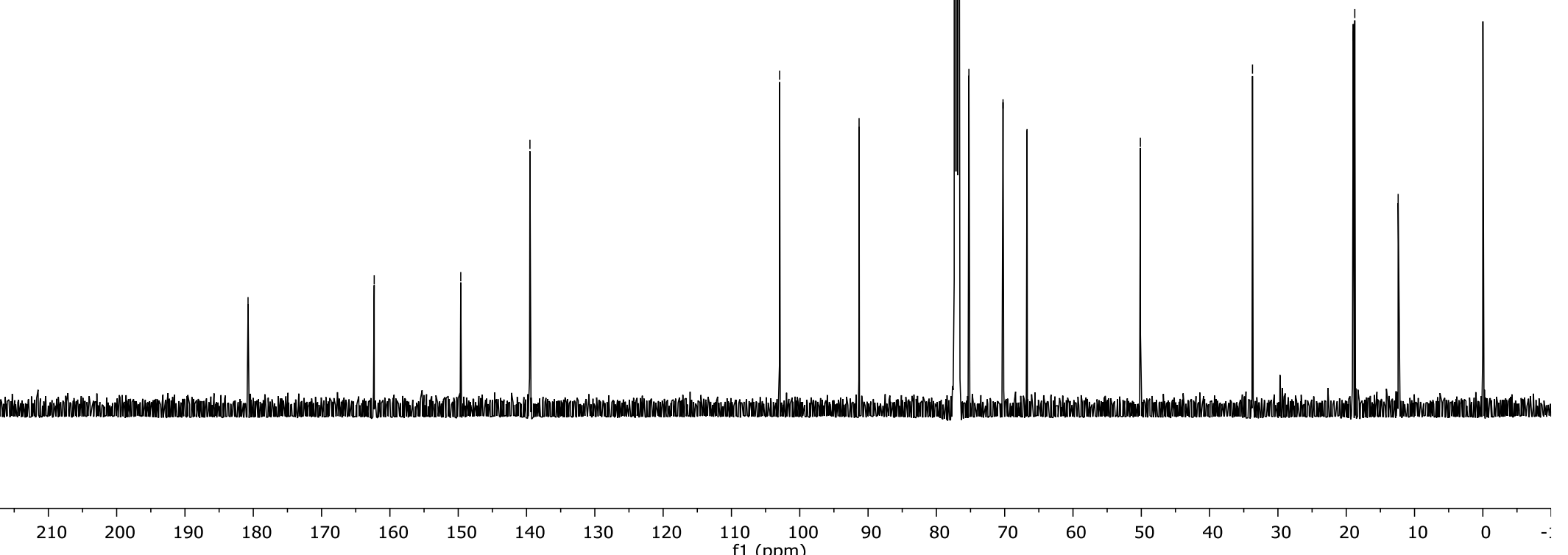

Figure S47. ${ }^{13} \mathrm{C}$ NMR (126 MHz, $\left.\mathrm{CDCl}_{3}\right)$ spectrum of compound 12. 


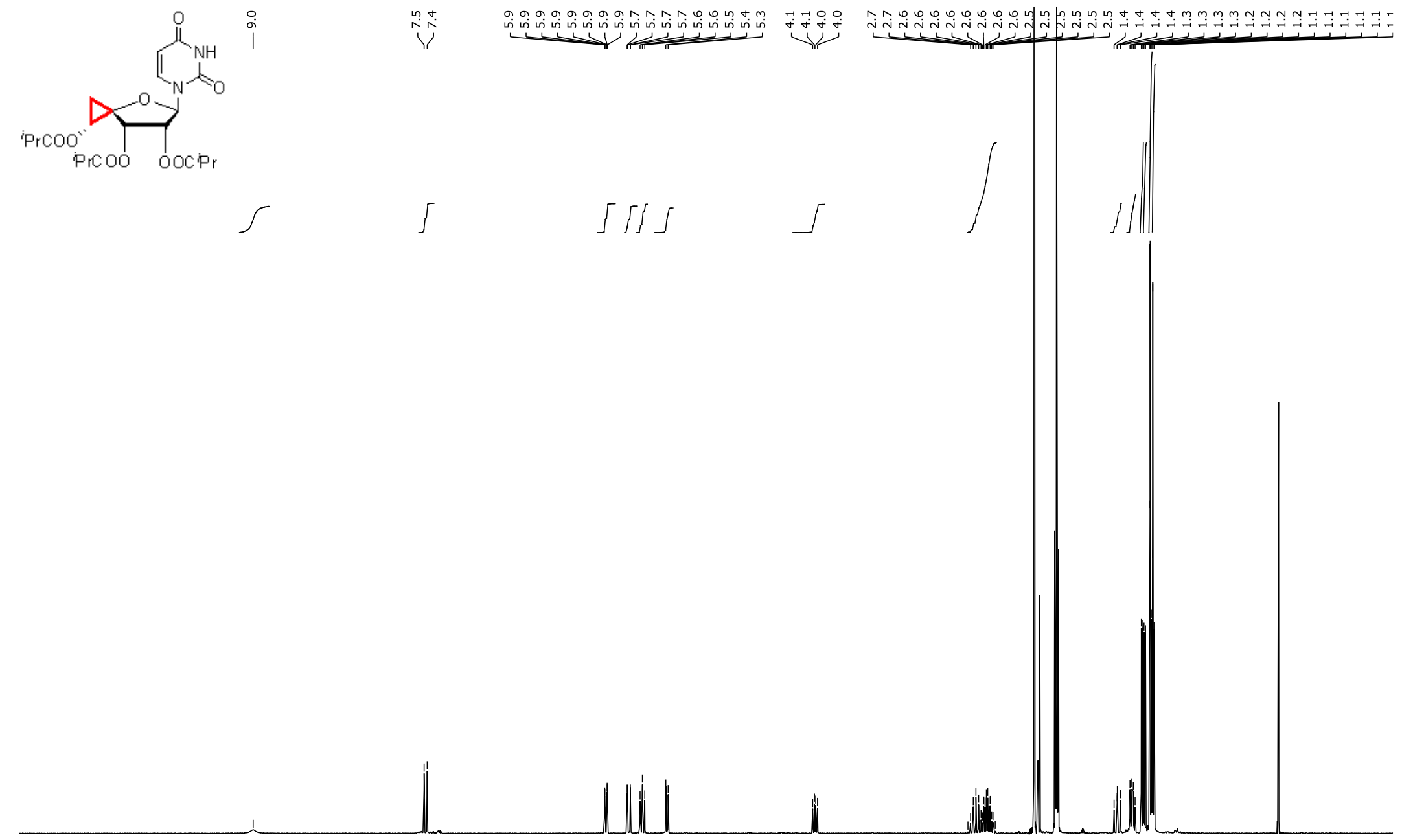

$\begin{array}{lllllllllllllllllllllllllll}10.5 & 10.0 & 9.5 & 9.0 & 8.5 & 8.0 & 7.5 & 7.0 & 6.5 & 6.0 & 5.5 & \begin{array}{c}5.0 \\ \mathrm{f} 1 \mathrm{(pm})\end{array} & 4.5 & 4.0 & 3.5 & 3.0 & 2.5 & 2.0 & 1.5 & 1.0 & 0.5 & 0.0 & -0.5 & -1\end{array}$

Figure S48. ${ }^{1} \mathrm{H}$ NMR $\left(300 \mathrm{MHz}, \mathrm{CDCl}_{3}\right)$ spectrum of compound 13. 

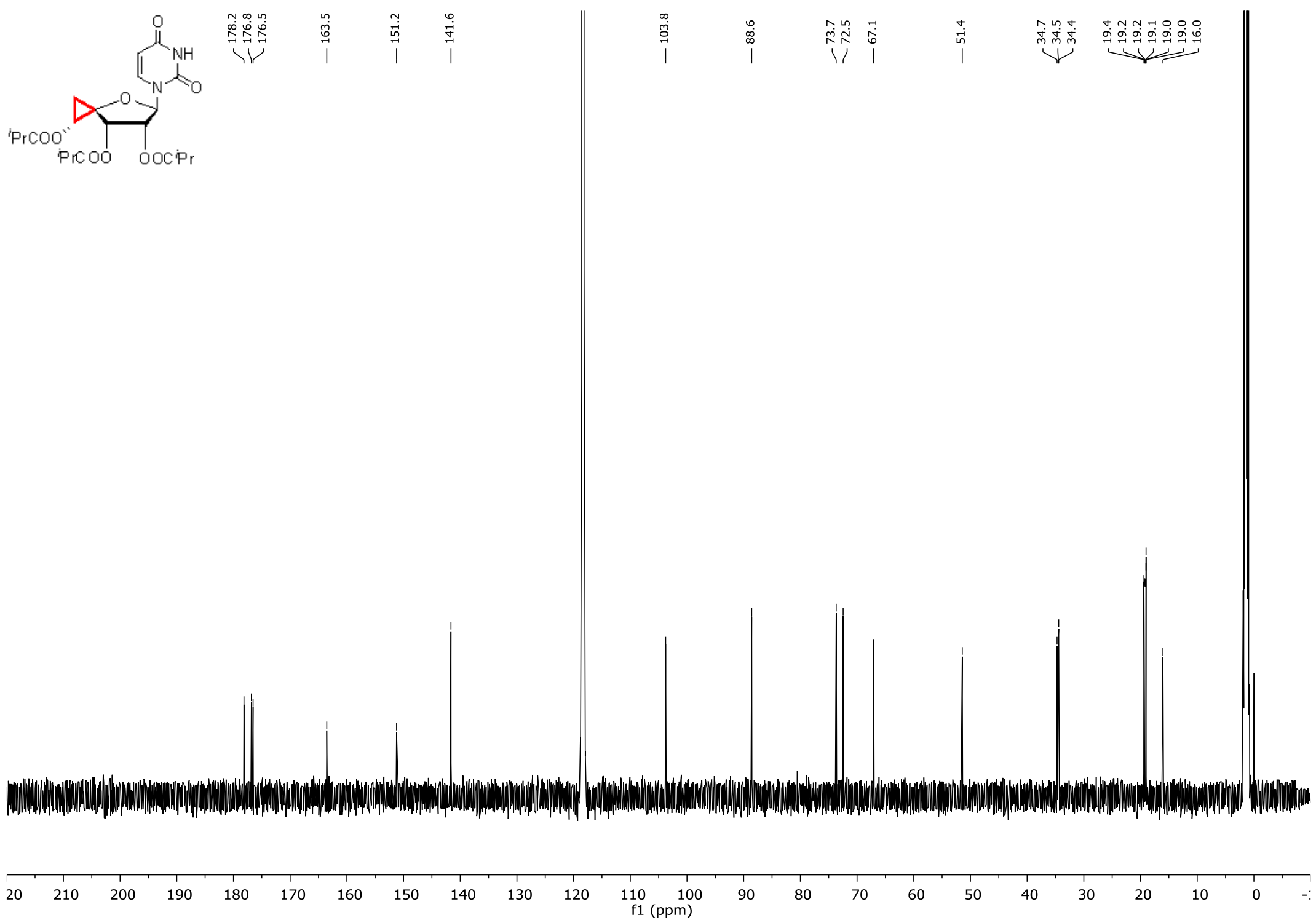

Figure S49. ${ }^{1} \mathrm{H}$ NMR (126 MHz, $\left.\mathrm{CDCl}_{3}\right)$ spectrum of compound 13. 
D-Ribose Series 


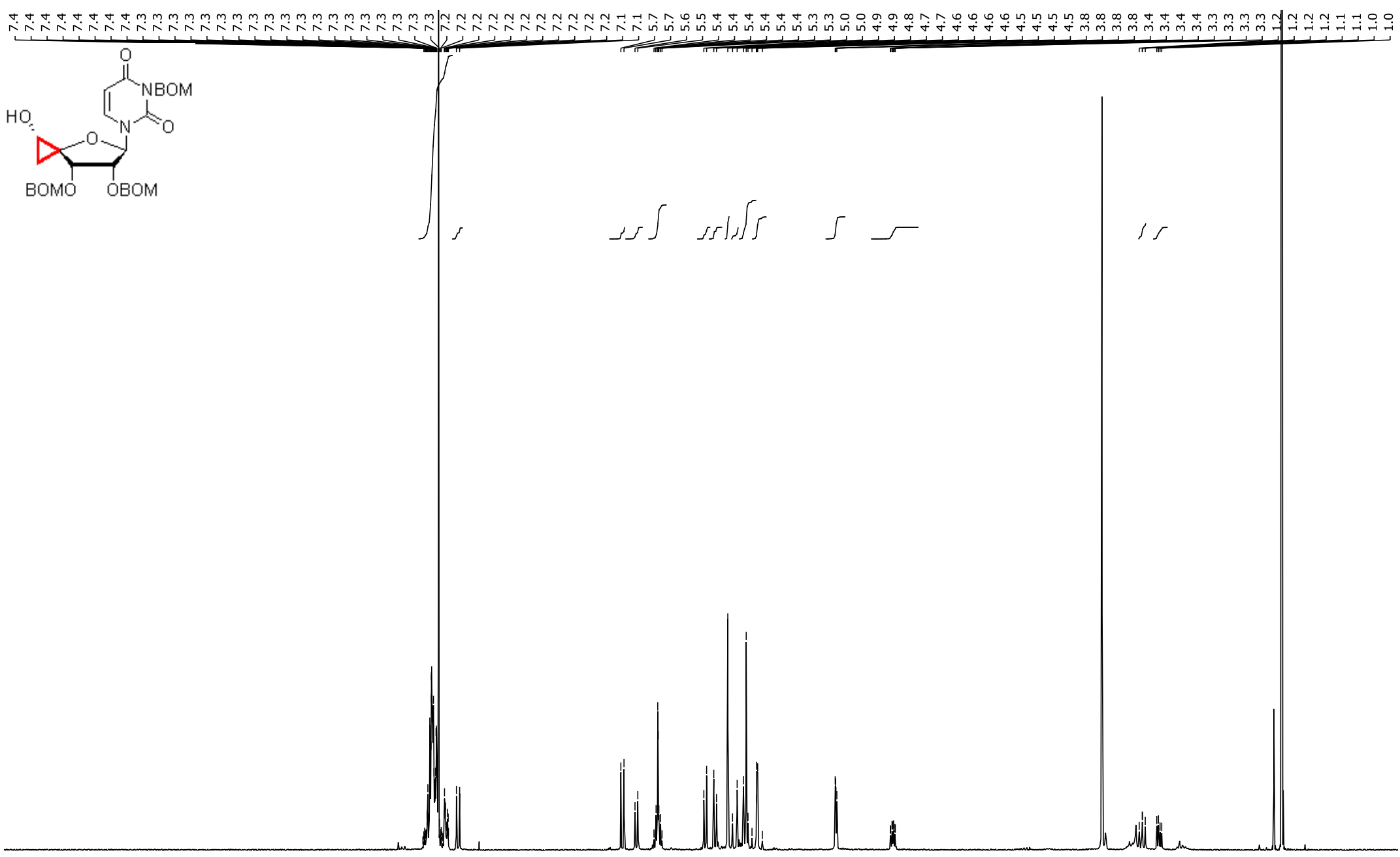

$1.0+10.5+10.0$

Figure S50. ${ }^{1} \mathrm{H}$ NMR (300 MHz, $\left.\mathrm{CDCl}_{3}\right)$ spectrum of compound 15. 


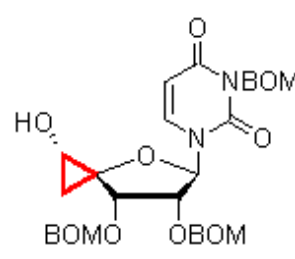

।

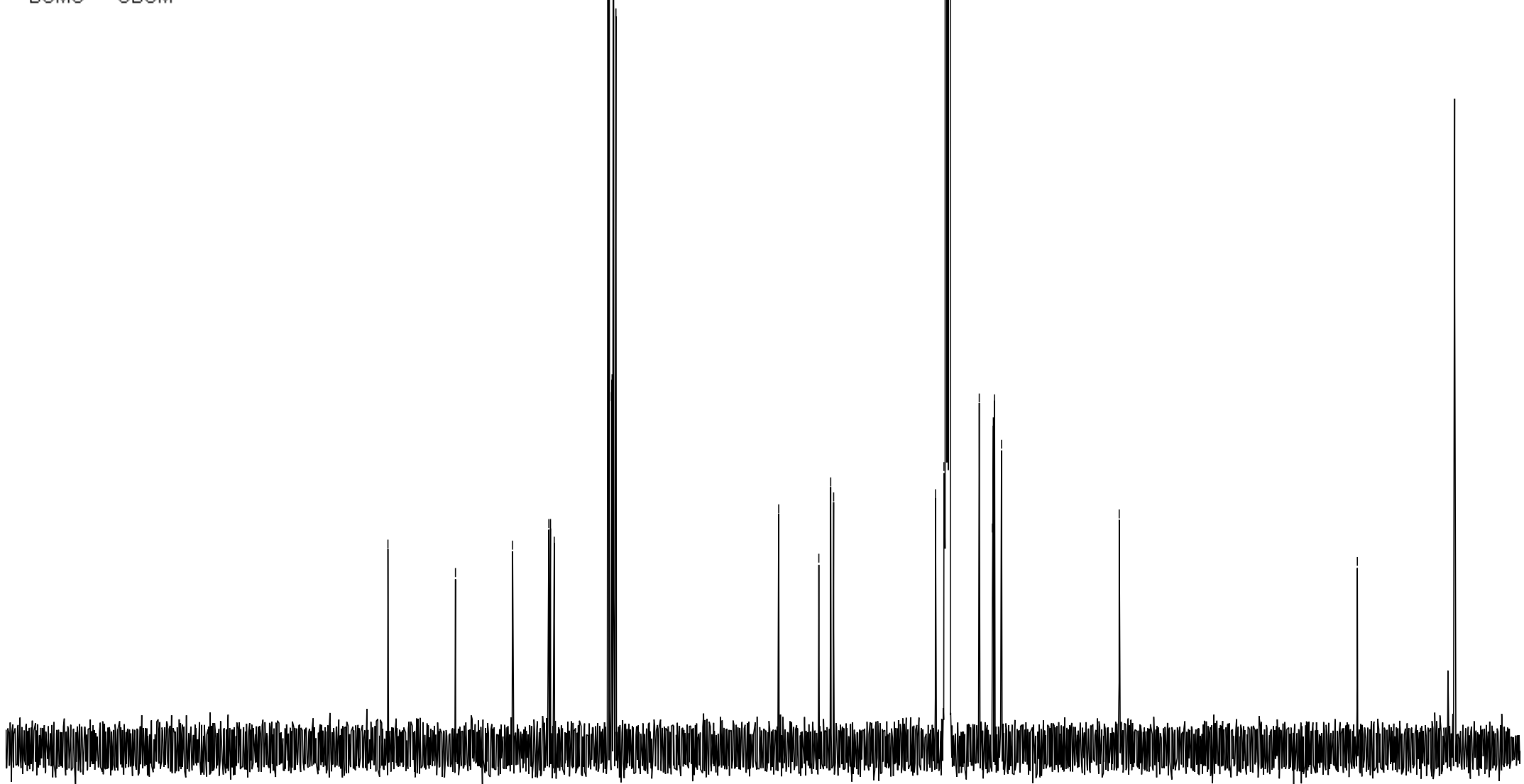

Figure S51. ${ }^{13} \mathrm{C}$ NMR (126 MHz, CDCl $)$ spectrum of compound 15. 


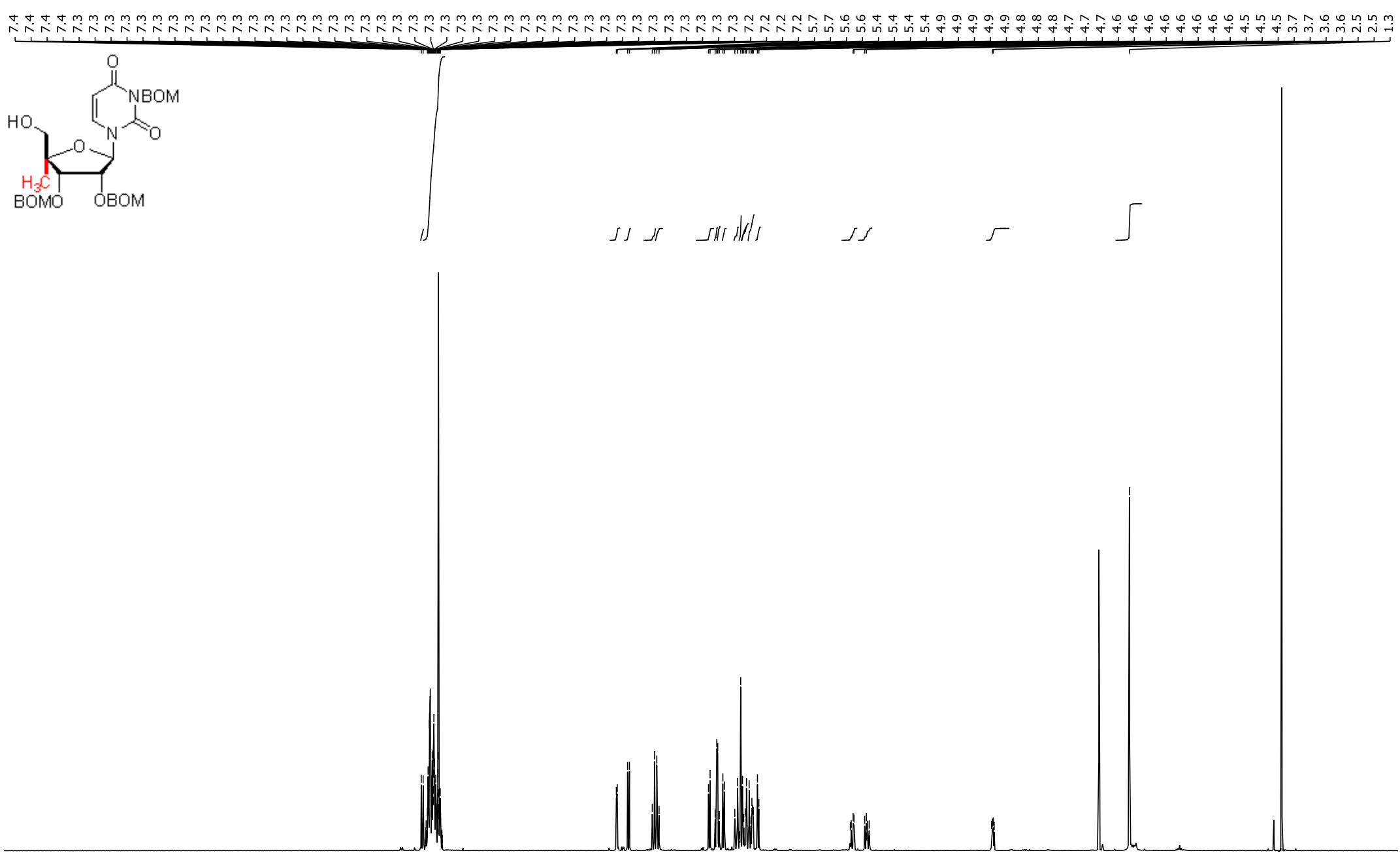

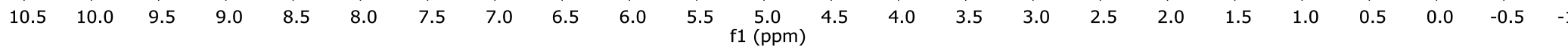

Figure S52. ${ }^{1} \mathrm{H}$ NMR $\left(500 \mathrm{MHz}, \mathrm{CDCl}_{3}\right)$ spectrum of compound 16. 


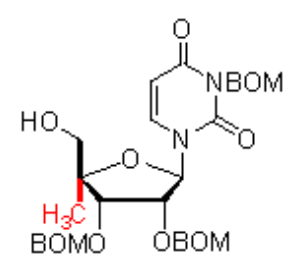

BOMO OBOM
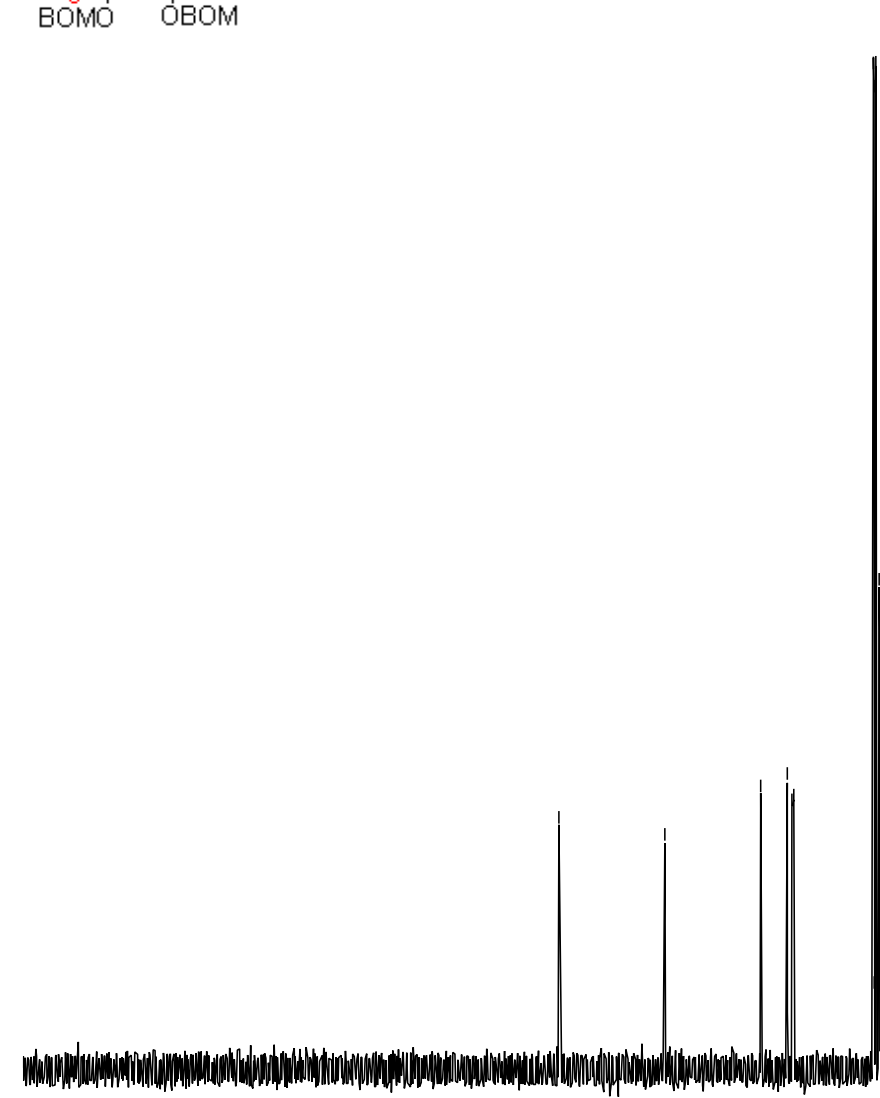

20

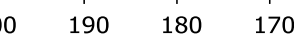

160

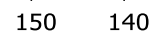

$130 \quad 120$

$110 \quad 100$

80

60

50

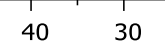

20

10

Figure S53. ${ }^{13} \mathrm{C}$ NMR $\left(126 \mathrm{MHz}, \mathrm{CDCl}_{3}\right)$ spectrum of compound 16. 

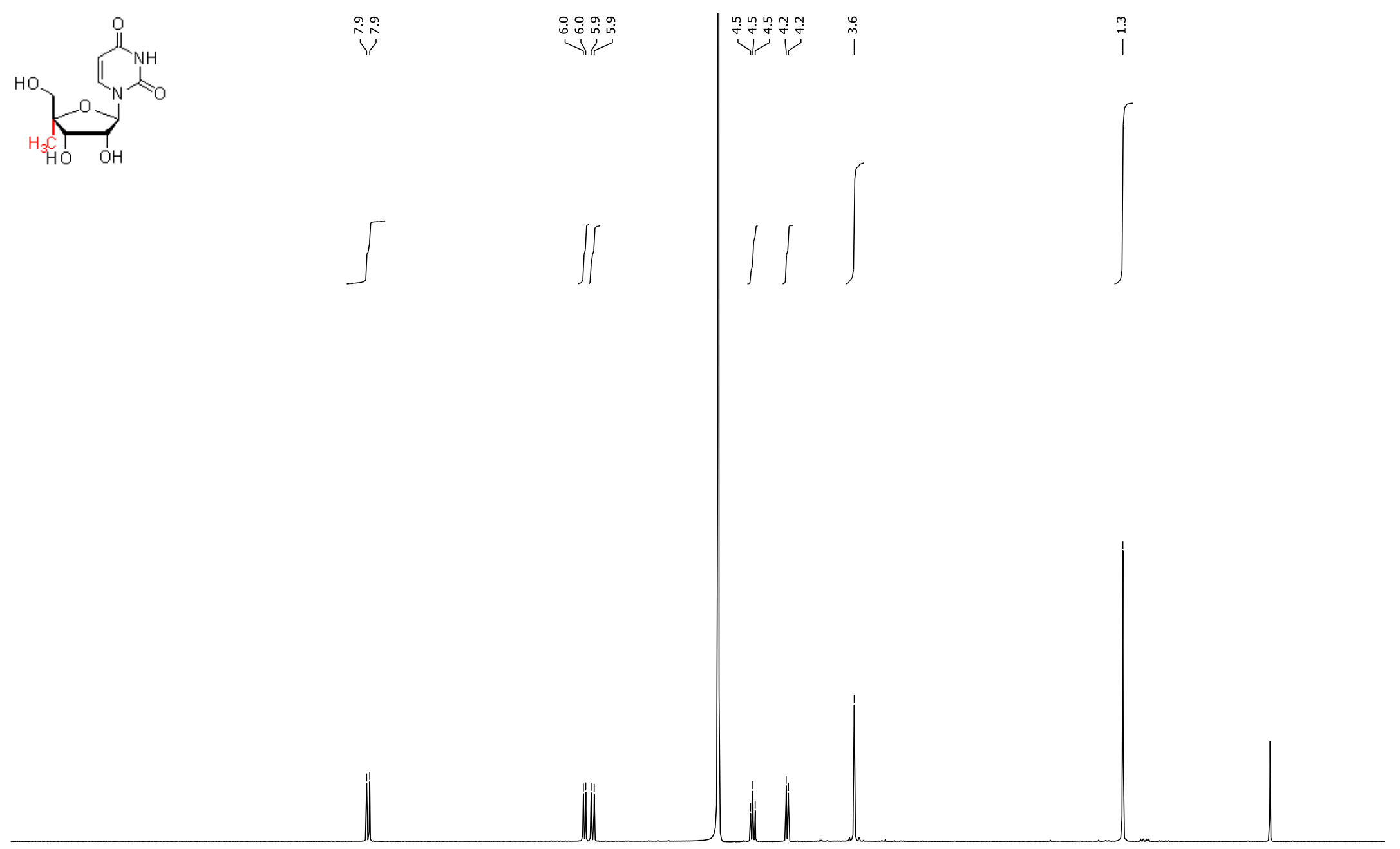

\begin{tabular}{lllllll}
\hline L. & 10.5 & 10.0 & 9.5 & 9.0 & 8.5
\end{tabular}

6.0

$5 \begin{gathered}5.0 \\ \mathrm{f} 1(\mathrm{ppm})\end{gathered}$

Figure S54. ${ }^{1} \mathrm{H}$ NMR $\left(300 \mathrm{MHz}, \mathrm{D}_{2} \mathrm{O}\right)$ spectrum of compound 17. 

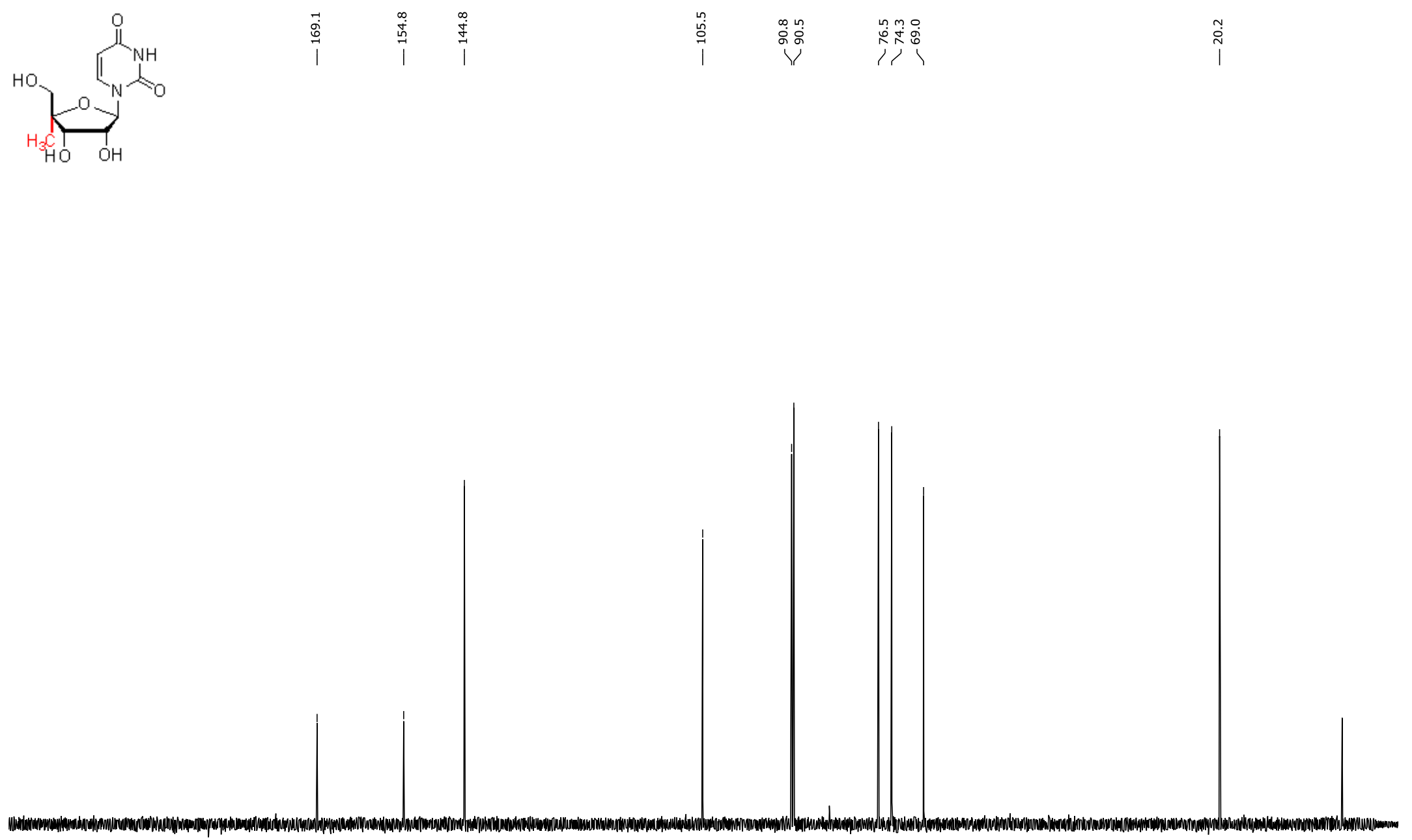

20

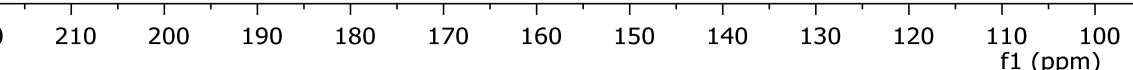

Figure S55. ${ }^{13} \mathrm{C}$ NMR (300 MHz, $\mathrm{D}_{2} \mathrm{O}$ ) spectrum of compound 17. 


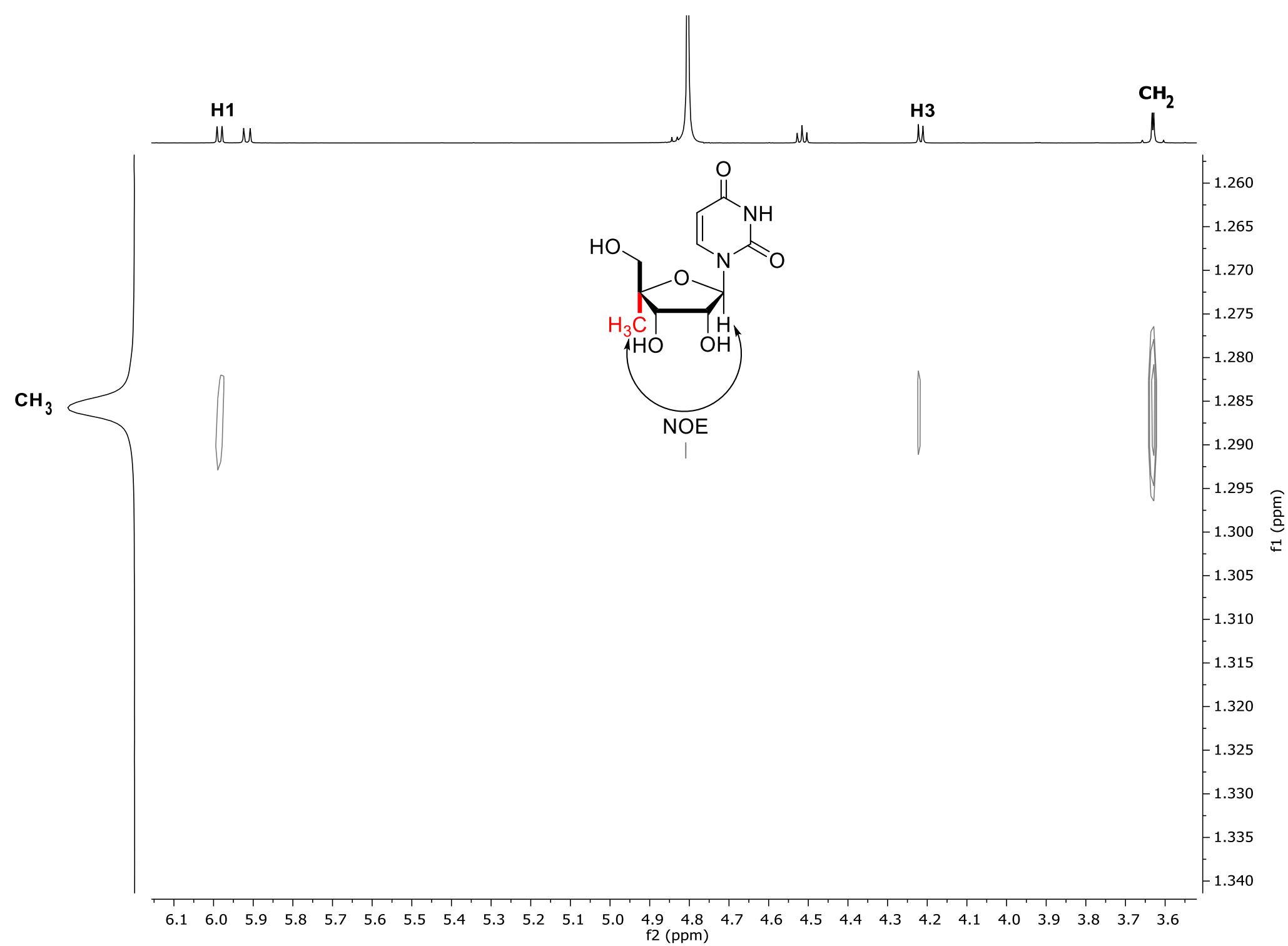

Figure S56. Partial NOESY (500 MHz, $\mathrm{D}_{2} \mathrm{O}$ ) of compound 17. 

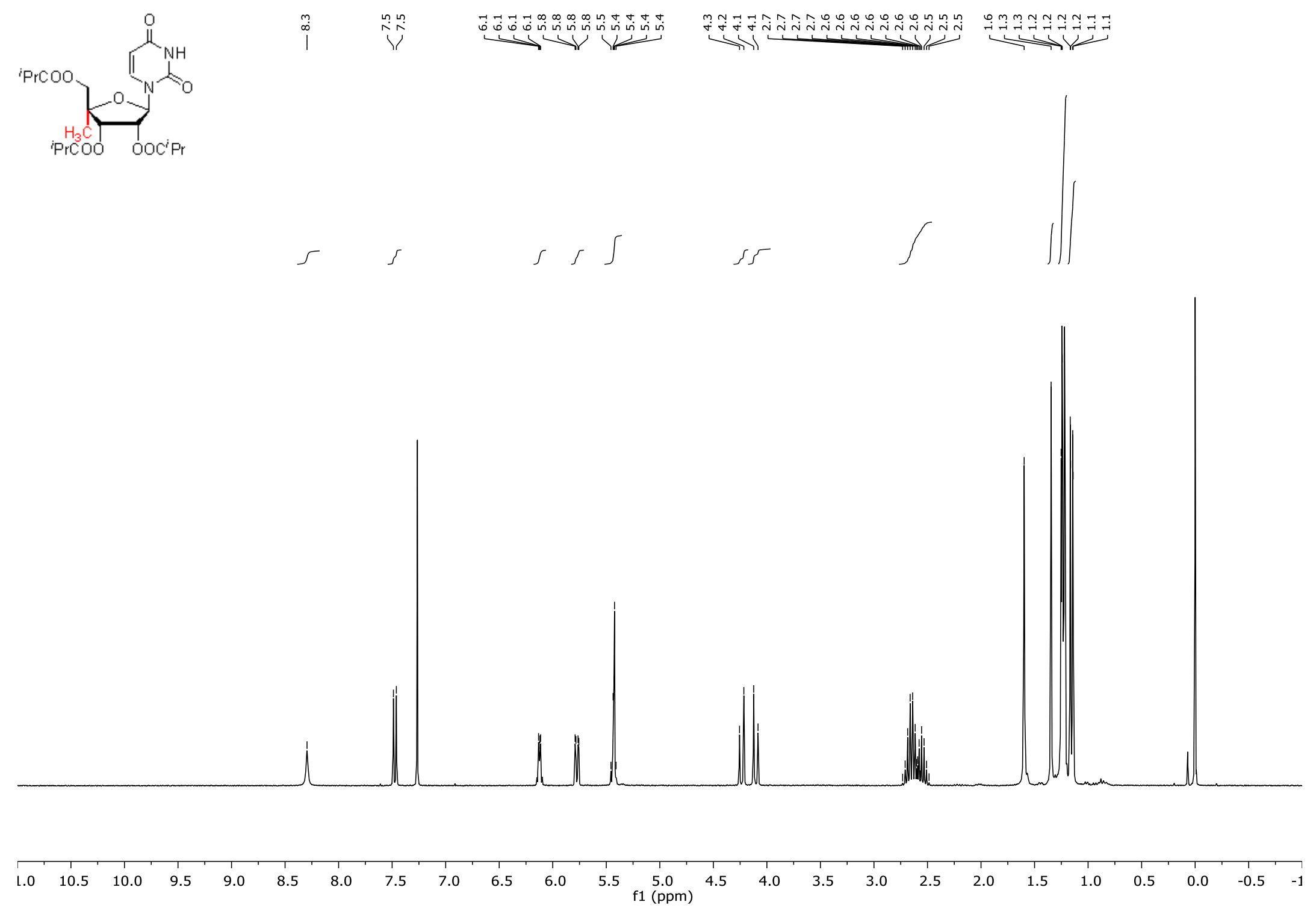

Figure S57. ${ }^{1} \mathrm{H}$ NMR $\left(300 \mathrm{MHz}, \mathrm{CDCl}_{3}\right)$ spectrum of compound 18. 


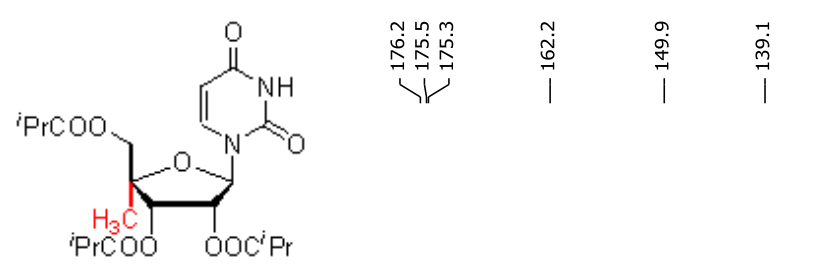

$\underbrace{\dot{\dot{p}}}$

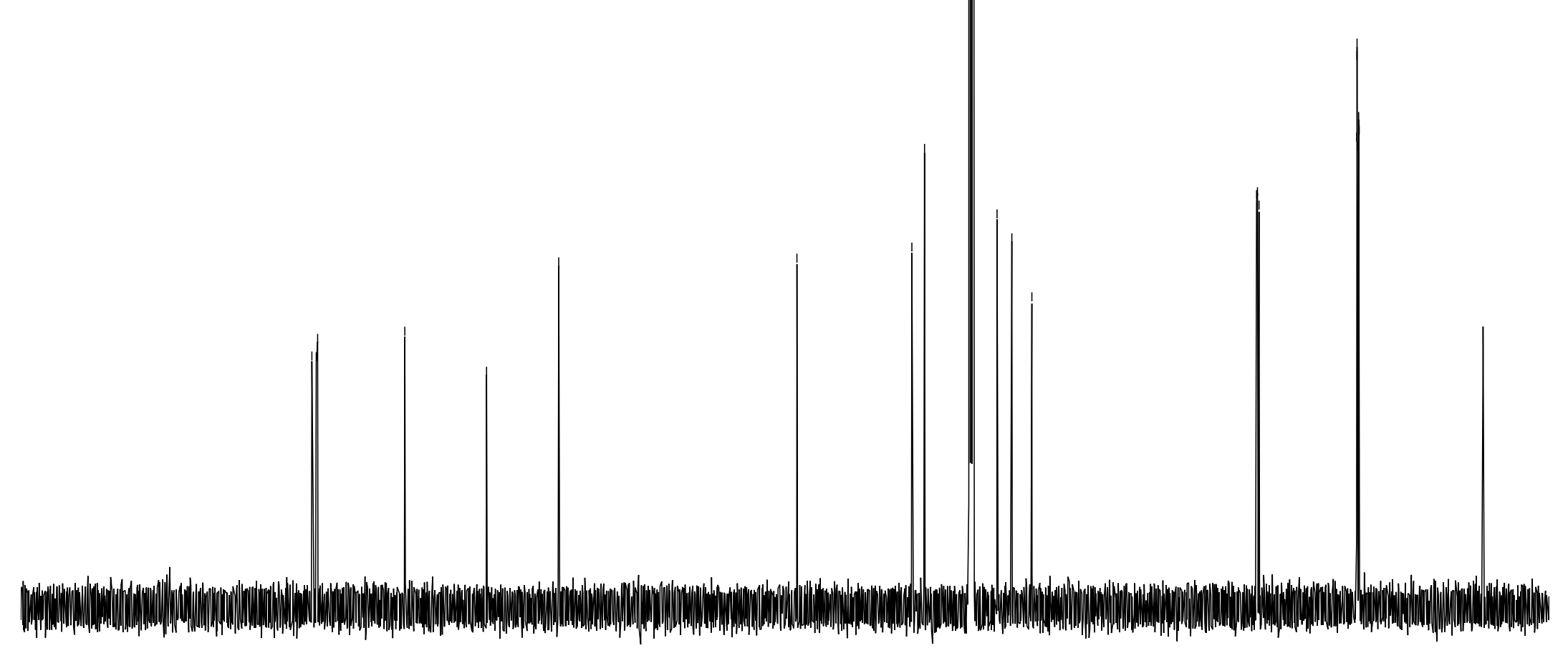

20

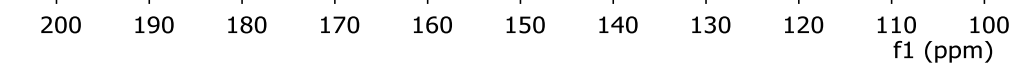

Figure S58. ${ }^{1} \mathrm{H}$ NMR $\left(126 \mathrm{MHz}, \mathrm{CDCl}_{3}\right)$ spectrum of compound 18. 


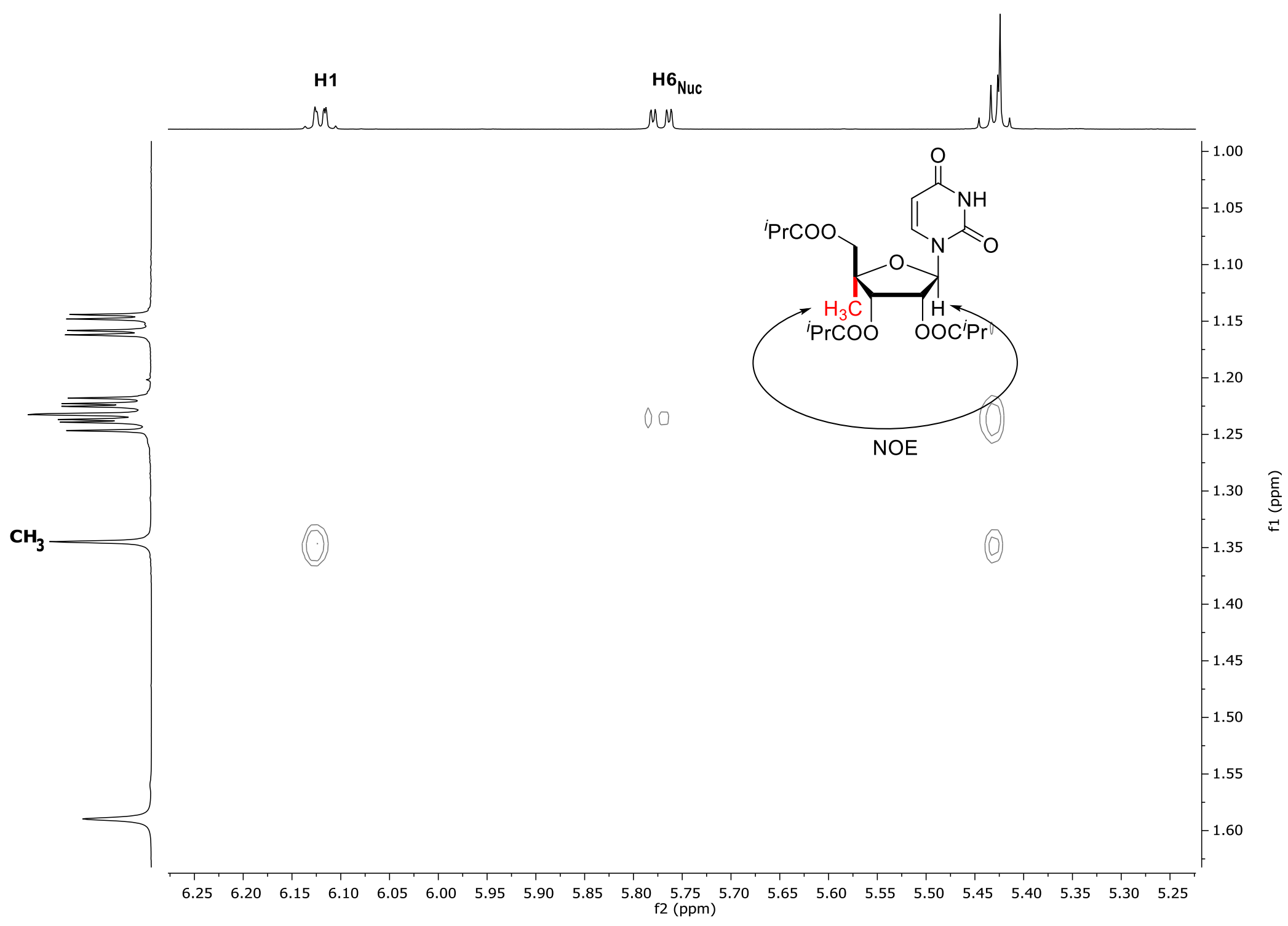

Figure S59. Partial NOESY ( $\left.500 \mathrm{MHz}, \mathrm{CDCl}_{3}\right)$ of compound 18. 


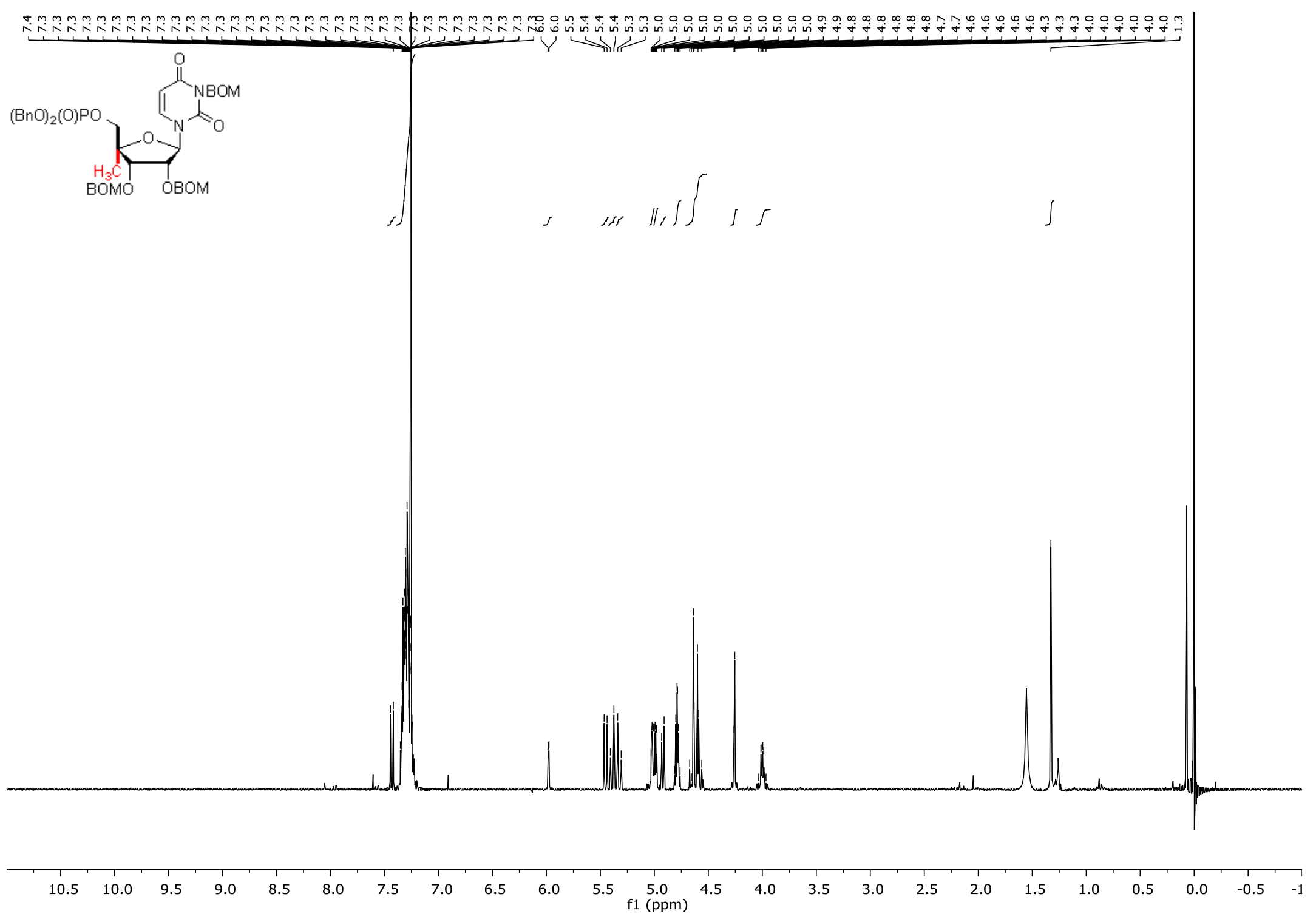

Figure S60. ${ }^{1} \mathrm{H} \mathrm{NMR}\left(300 \mathrm{MHz}, \mathrm{CDCl}_{3}\right)$ spectrum of compound 19. 


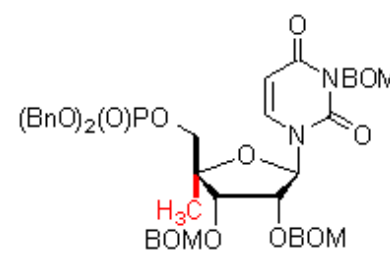

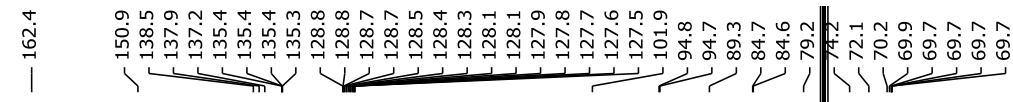

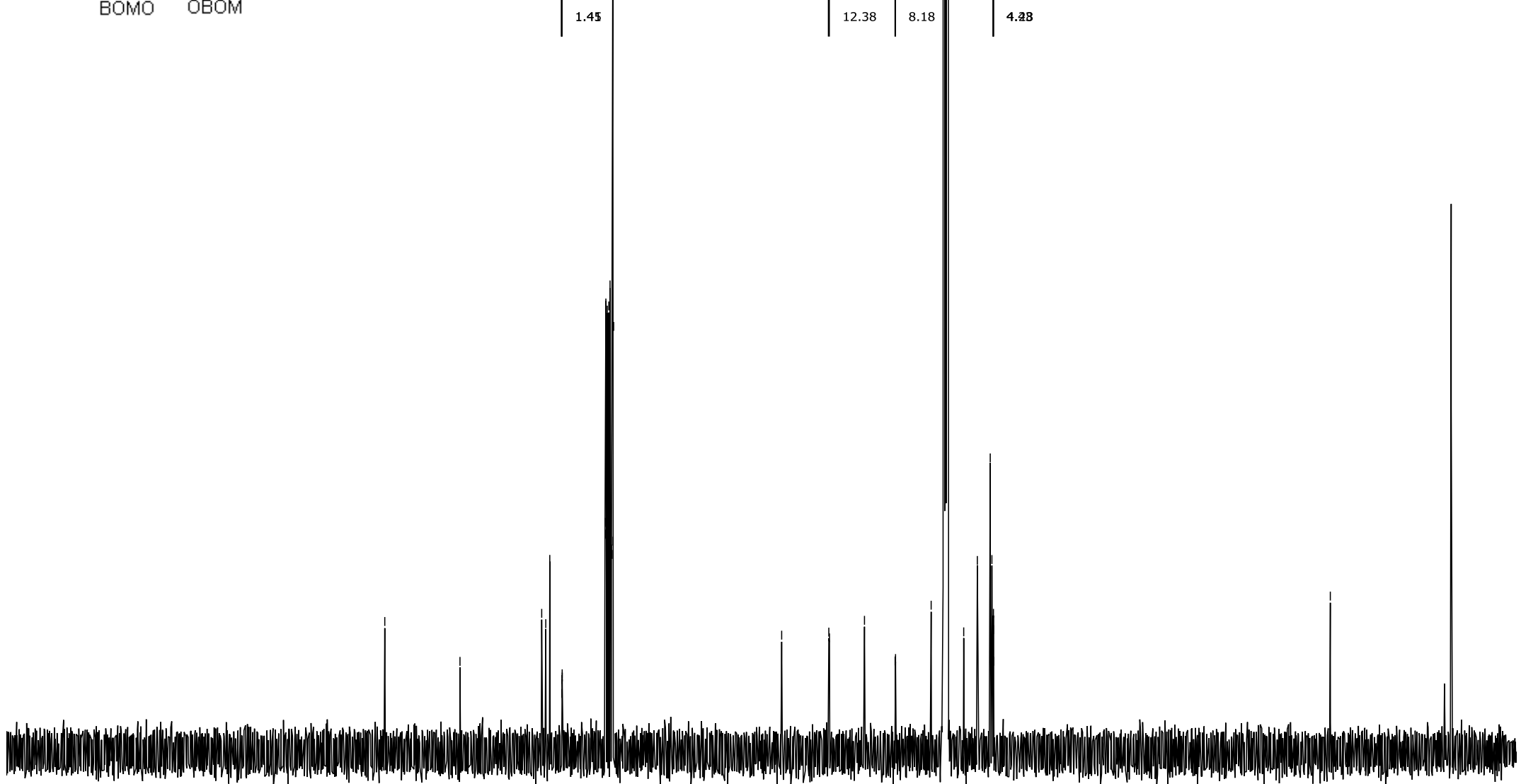

Figure S61. ${ }^{13} \mathrm{C}$ NMR (126 MHz, $\mathrm{CDCl}_{3}$ ) spectrum of compound 19. 


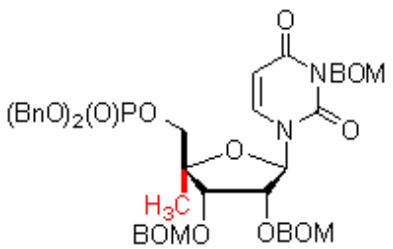

Figure S62. ${ }^{31} \mathrm{P}$ NMR (122 MHz, $\mathrm{CDCl}_{3}$ ) spectrum of compound 19. 

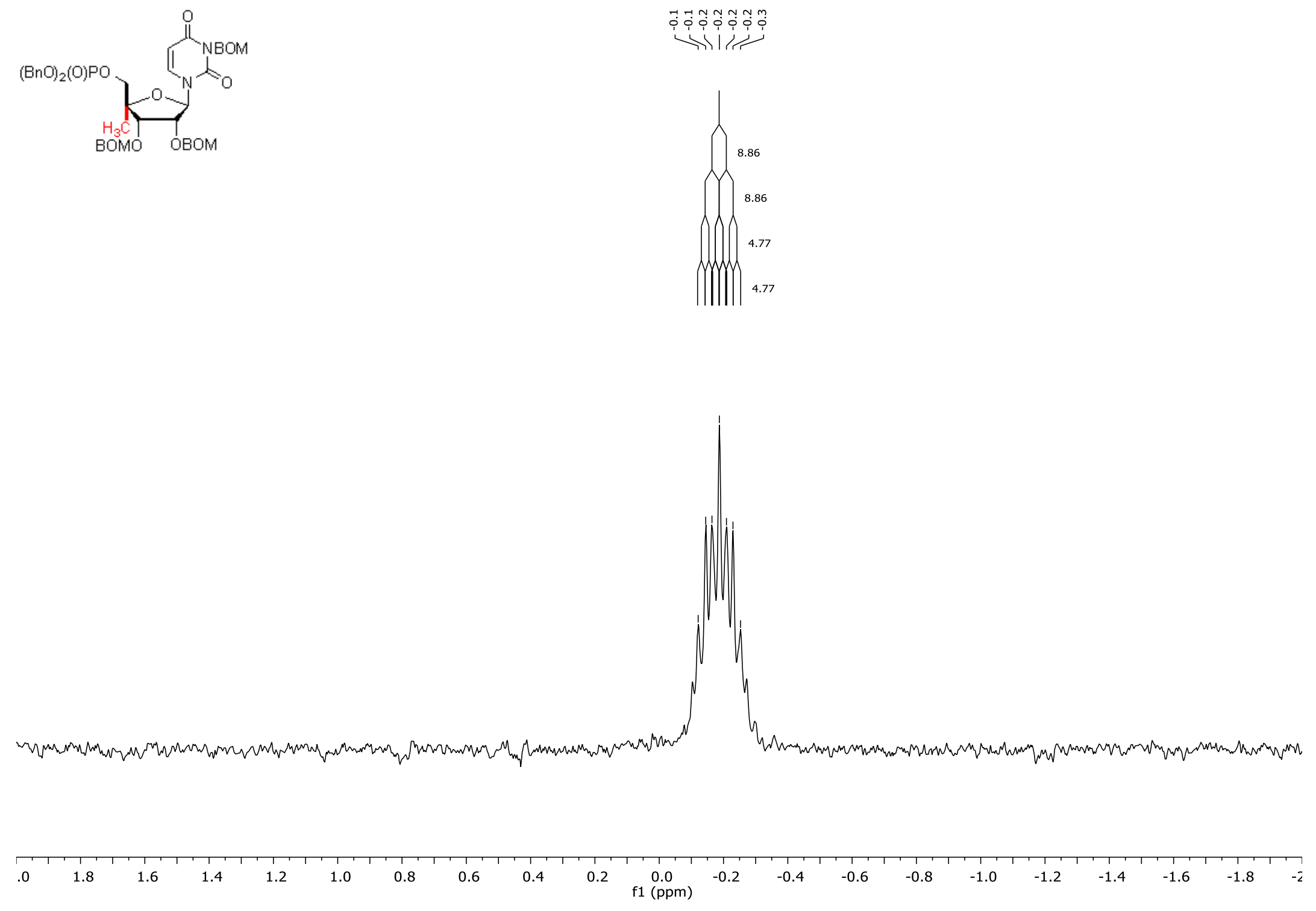

Figure S63. ${ }^{31} \mathrm{P} \mathrm{NMR}\left(122 \mathrm{MHz}, \mathrm{CDCl}_{3}\right.$ ) spectrum of compound 19 (no ${ }^{1} \mathrm{H}$ decoupling). 

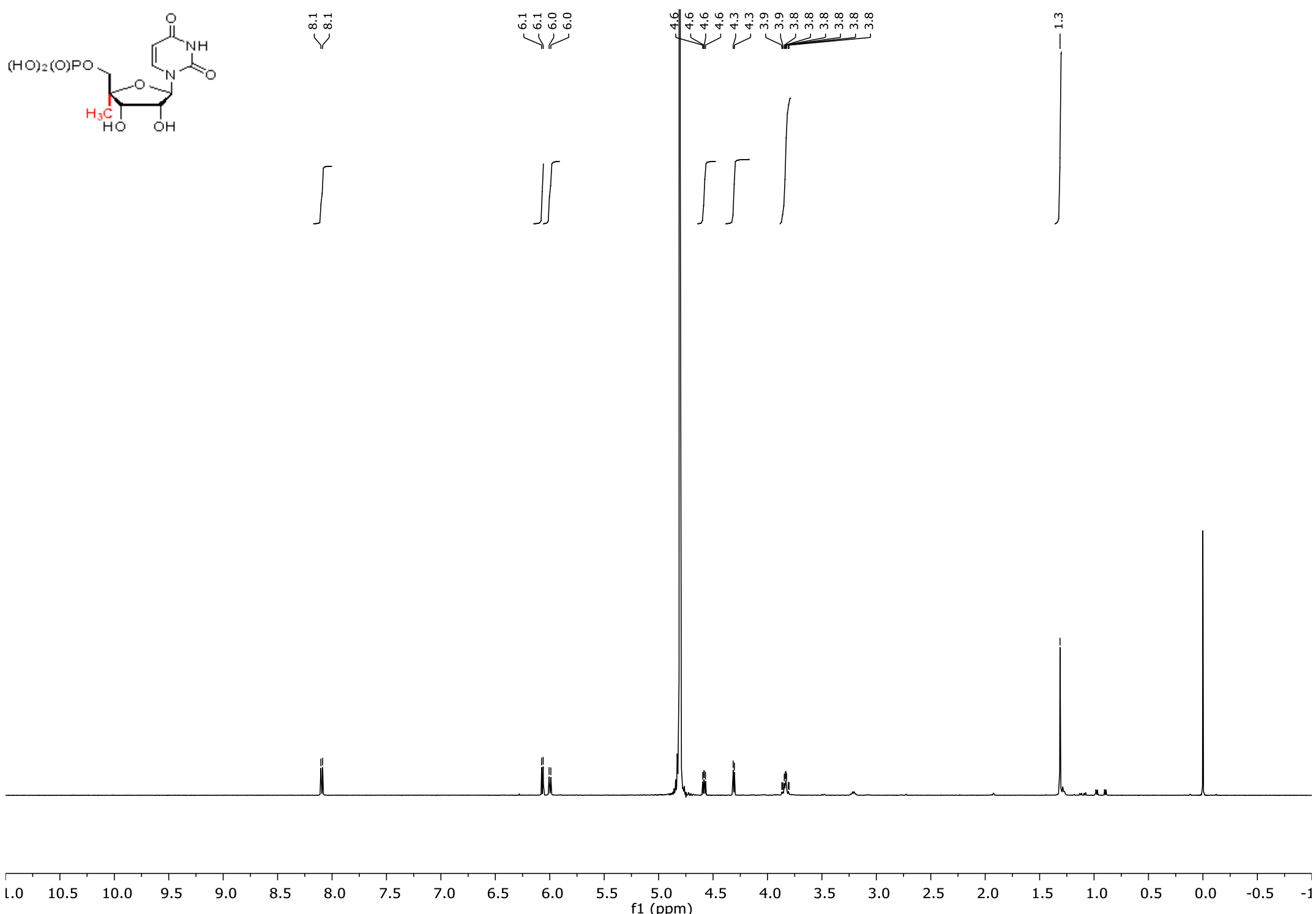

Figure S64. ${ }^{1} \mathrm{H}$ NMR $\left(500 \mathrm{MHz}, \mathrm{D}_{2} \mathrm{O}\right)$ spectrum of compound 20. 
$\left(\mathrm{HO}_{2}(\mathrm{O}) \mathrm{PO}\right.$
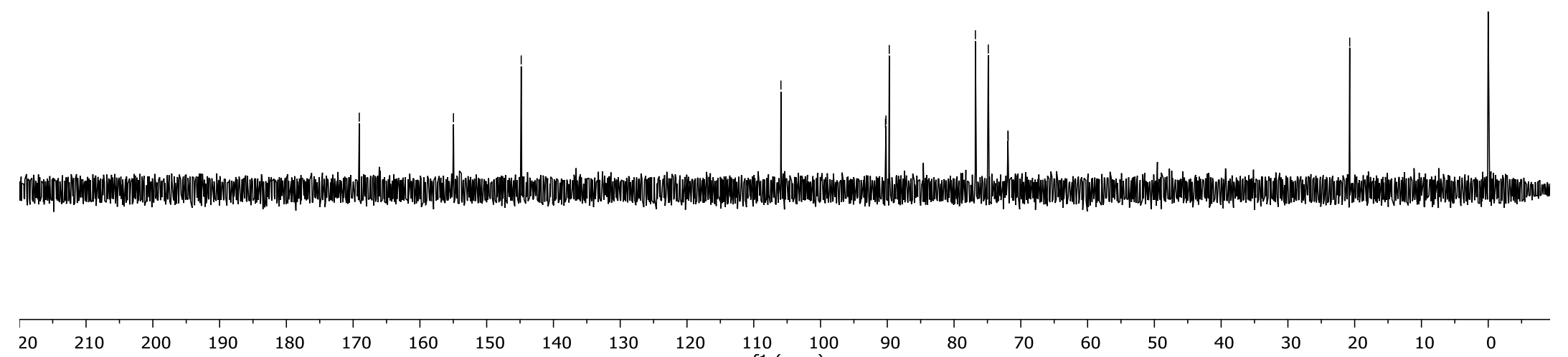

$\begin{array}{llll}190 & 180 & 170\end{array}$

160

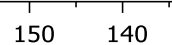

$130 \quad 120$

$110 \quad 100$

90

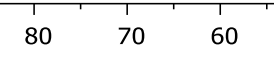

50

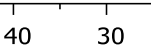

20

Figure S65. ${ }^{13} \mathrm{C}$ NMR (126 MHz, $\mathrm{D}_{2} \mathrm{O}$ ) spectrum of compound 20. 

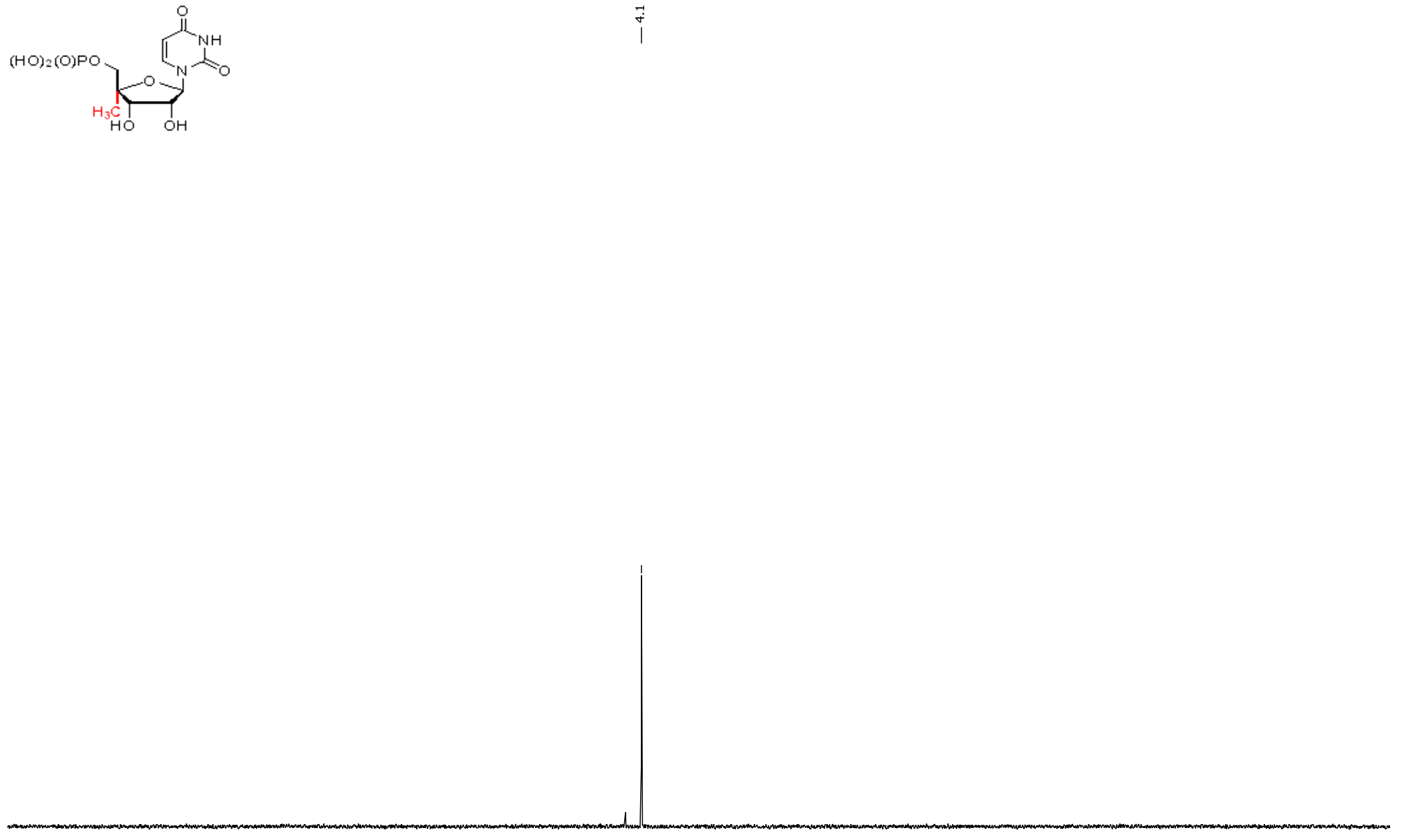

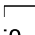

35

30

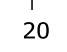

15

10

f1 (ppm)

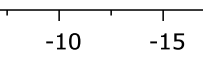

$-20$

$-25$

$-30$

$\begin{array}{ll}-35 & -40\end{array}$

Figure S66. ${ }^{31} \mathrm{P} \mathrm{NMR}\left(122 \mathrm{MHz}, \mathrm{D}_{2} \mathrm{O}\right.$ ) spectrum of compound 20 (signal enhanced by addition of 2 drops of $\mathrm{NEt} / 3 / 0.6 \mathrm{~mL}$ $\mathrm{D} 2 \mathrm{O})$. 


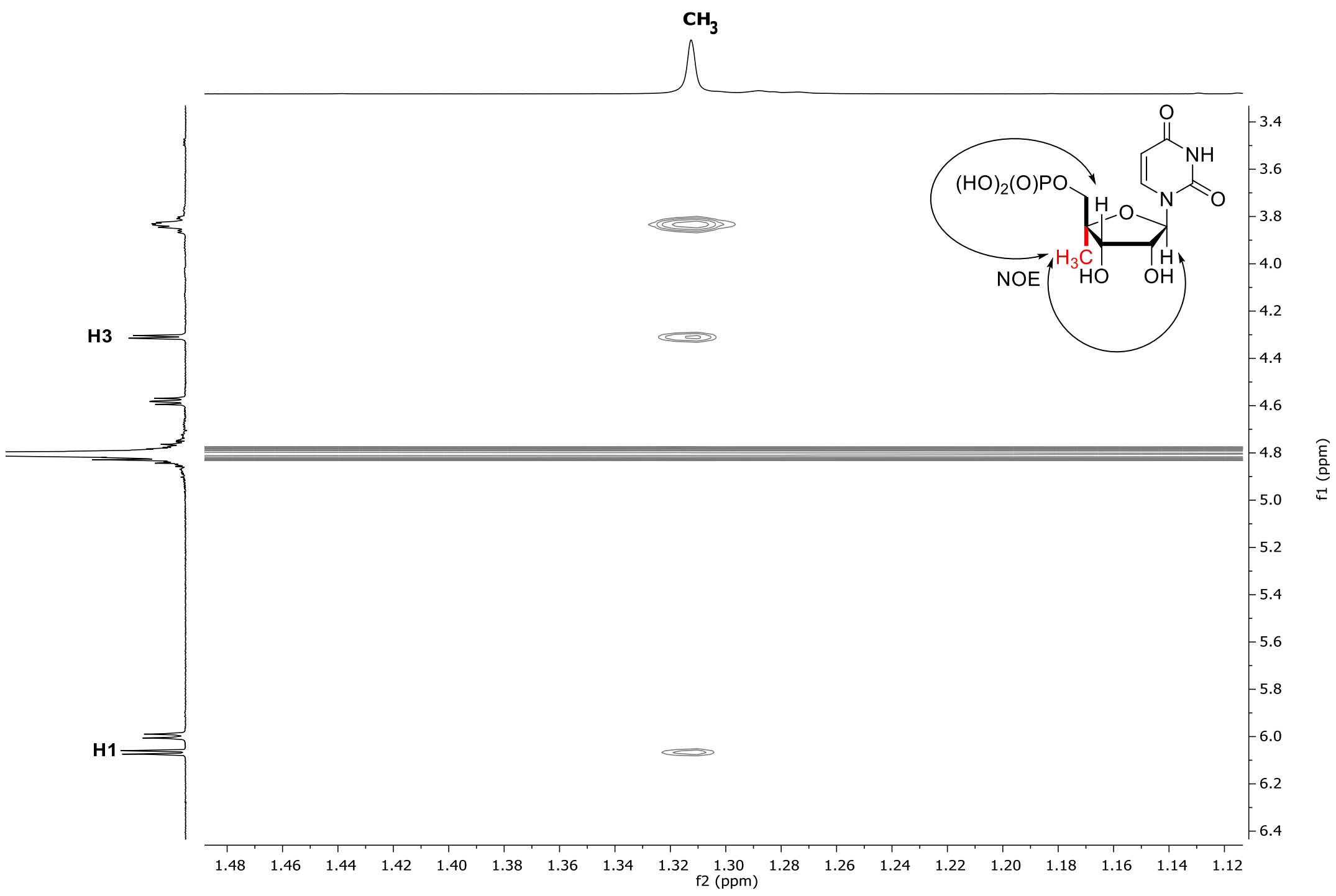

Figure S67. Partial NOESY (500 MHz, $\left.\mathrm{D}_{2} \mathrm{O}\right)$ of compound 20. 

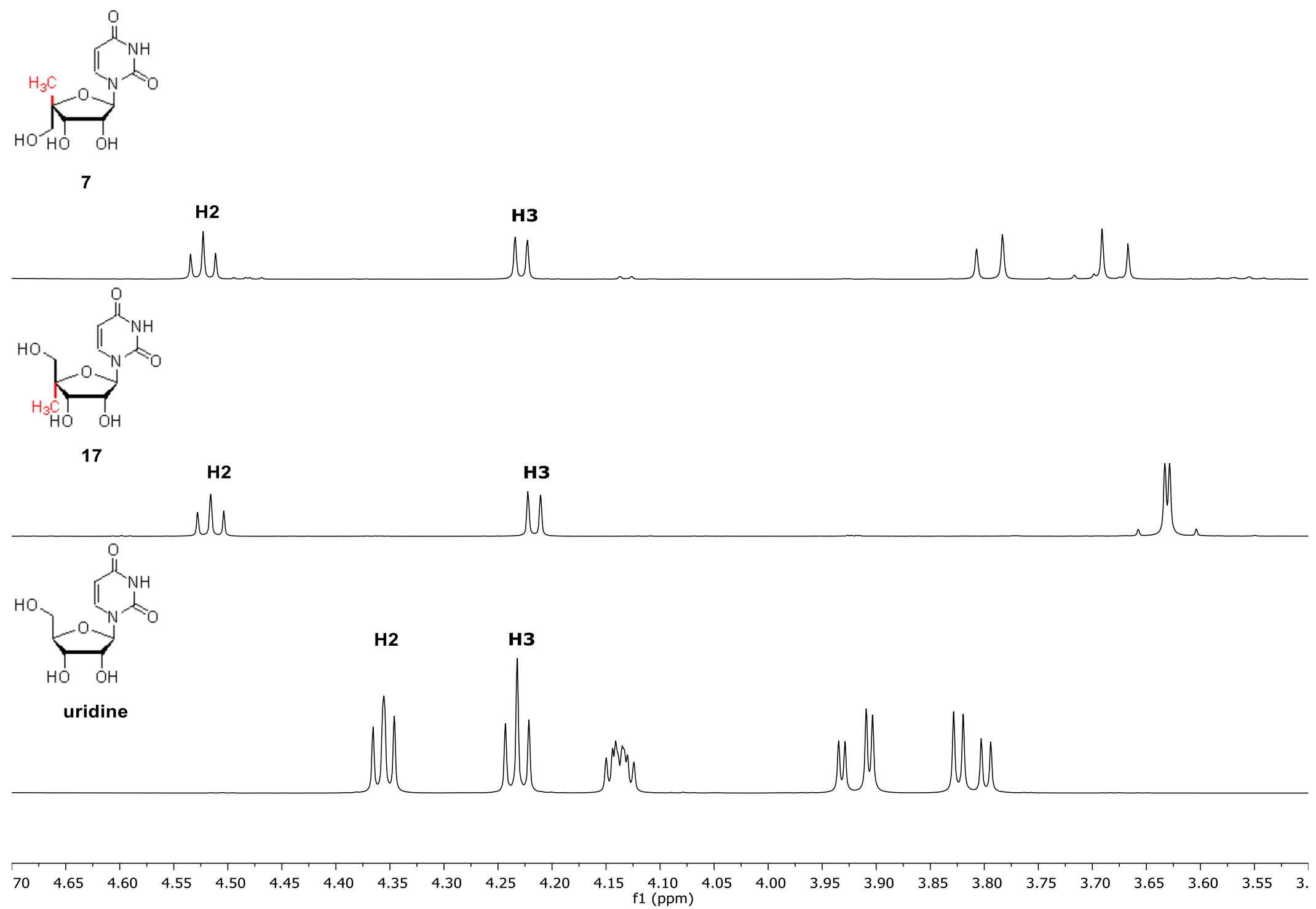

Figure S68. ${ }^{1} \mathrm{H}$ NMR $\left(300 \mathrm{MHz}, \mathrm{D}_{2} \mathrm{O}\right.$ ) Detailed comparison between both $\mathrm{C} 4$ '-methylated derivatives 7 and 17 and native uridine. 

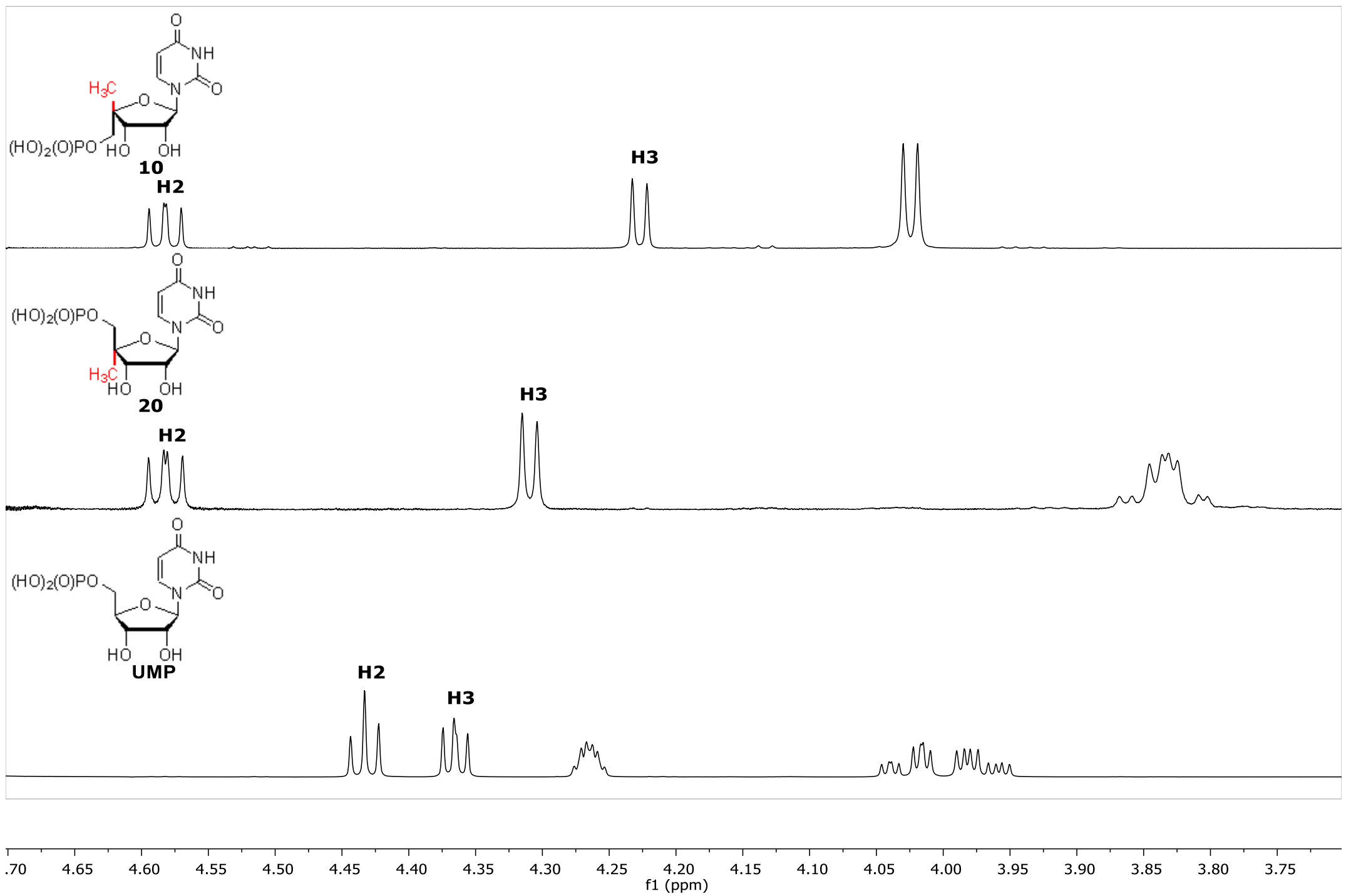

Figure S69. ${ }^{1} \mathrm{H}$ NMR (300 MHz, $\mathrm{D}_{2} \mathrm{O}$ ) Detailed comparison between both $\mathrm{C} 4$ '-methylated 5'-monophosphates 10 and 20 and native UMP. 


\section{X-Ray Crystallographic Data}

\section{Crystal Structure Determinations}

Crystals were mounted in inert oil on nylon loops and transferred to the cold gas stream of the diffractometer (Rigaku/Oxford XtaLAB Synergy using mirror-focussed $\mathrm{Cu}$ Ka radiation). Absorption corrections were implemented on the basis of multi-scans. The structures were refined anisotropically on $F^{2}$ using the program SHELXL-20171. Hydrogen atoms of $\mathrm{OH}$ and $\mathrm{NH}$ groups were refined freely; other hydrogens were included using rigid methyl groups or a riding model starting from calculated positions. The absolute configuration was confirmed for each structure by the Flack parameter. Special features: For compound $\mathbf{1 b}$, the $U$ values of atom $\mathrm{O} 4$ are rather high.

Crystallographic data are summarized in Table S1. Additionally, complete data have been deposited with the Cambridge Crystallographic Data Centre under the numbers CCDC 1971611-1971614. Copies of the data can be obtained free of charge from www.ccdc.cam.ac.uk/data_request/cif.

Thermal ellipsoid plots (all at the $50 \%$ probability level). 
Table S1. Crystallographic Data and Structure Refinement Details for Compounds 1a, 1b, 11 and 17.

\begin{tabular}{|c|c|c|c|c|}
\hline Compound & $1 a$ & $1 \mathrm{~b}$ & 11 & 17 \\
\hline CCDC number & 1971611 & 1971612 & 1971613 & 1971614 \\
\hline Formula & $\mathrm{C}_{15} \mathrm{H}_{20} \mathrm{~N}_{2} \mathrm{O}_{6}$ & $\mathrm{C}_{22} \mathrm{H}_{20} \mathrm{~N}_{2} \mathrm{O}_{6}$ & $\mathrm{C}_{27} \mathrm{H}_{26} \mathrm{~N}_{2} \mathrm{O}_{7}$ & $\mathrm{C}_{10} \mathrm{H}_{14} \mathrm{~N}_{2} \mathrm{O}_{6}$ \\
\hline$M_{r}$ & 324.33 & 408.40 & 490.50 & 258.23 \\
\hline Cryst. size (mm) & $0.15 \times 0.04 \times 0.02$ & $0.15 \times 0.12 \times 0.04$ & $0.15 \times 0.1 \times 0.1$ & $0.12 \times 0.1 \times 0.08$ \\
\hline Crystal system & orthorhombic & monoclinic & orthorhombic & monoclinic \\
\hline Space group & $P 2{ }_{1} 2_{1} 2_{1}$ & $P 2_{1}$ & $P 2{ }_{1}{ }_{1} 2_{1}$ & $P 2_{1}$ \\
\hline Temperature $\left({ }^{\circ} \mathrm{C}\right)$ & -173 & -173 & -173 & -173 \\
\hline$a(\AA)$ & $5.91240(8)$ & $5.99677(7)$ & $10.75286(7)$ & $8.35326(14)$ \\
\hline$b(\AA)$ & $6.26542(6)$ & $7.61343(8)$ & $10.83630(7)$ & $5.76025(11)$ \\
\hline$c(\AA)$ & $40.7670(4)$ & $20.4686(2)$ & $41.1130(3)$ & $11.5435(2)$ \\
\hline$\alpha\left(^{\circ}\right)$ & 90 & 90 & 90 & 90 \\
\hline$\beta\left({ }^{\circ}\right)$ & 90 & $92.4786(10)$ & 90 & $95.1416(16)$ \\
\hline$\gamma\left({ }^{\circ}\right)$ & 90 & 90 & 90 & 90 \\
\hline$V\left(\AA^{3}\right)$ & 1510.16 & 933.64 & 4970.54 & 553.20 \\
\hline$Z$ & 4 & 2 & 8 & 2 \\
\hline$D_{\mathrm{x}}\left(\mathrm{Mg} \mathrm{m}^{-3}\right)$ & 1.427 & 1.453 & 1.360 & 1.550 \\
\hline$\lambda(\AA)$ & 1.54184 & 1.54184 & 1.54184 & 1.54184 \\
\hline$\mu\left(\mathrm{mm}^{-1}\right)$ & 0.94 & 0.89 & 0.82 & 1.11 \\
\hline Transmissions & $0.857-1.000$ & $0.686-1.000$ & $0.850-1.000$ & $0.895-1.000$ \\
\hline$F(000)$ & 688 & 428 & 2064 & 272 \\
\hline $2 \theta_{\max }$ & 154.9 & 156.0 & 155.6 & 155.1 \\
\hline Refl. measured & 59504 & 58527 & 217865 & 22406 \\
\hline Refl. indep. & 3181 & 3967 & 10099 & 2297 \\
\hline$R_{\text {int }}$ & 0.043 & 0.044 & 0.043 & 0.031 \\
\hline Parameters & 216 & 279 & 662 & 181 \\
\hline Restraints & 0 & 1 & 0 & 1 \\
\hline$w R\left(F^{2}\right.$, all refl. $)$ & 0.063 & 0.088 & 0.061 & 0.058 \\
\hline$R(F,>4 \sigma(F))$ & 0.025 & 0.034 & 0.024 & 0.022 \\
\hline Flack parameter & $-0.01(3)$ & $0.01(4)$ & $-0.018(19)$ & $-0.03(5)$ \\
\hline$S$ & 1.04 & 1.03 & 1.02 & 1.07 \\
\hline $\operatorname{Max} . \Delta p\left(\mathrm{e} \AA^{-3}\right)$ & $0.16,-0.19$ & $0.31,-0.25$ & $0.22,-0.16$ & $0.19,-0.16$ \\
\hline
\end{tabular}




\section{X-Ray Data for Compound 1a}

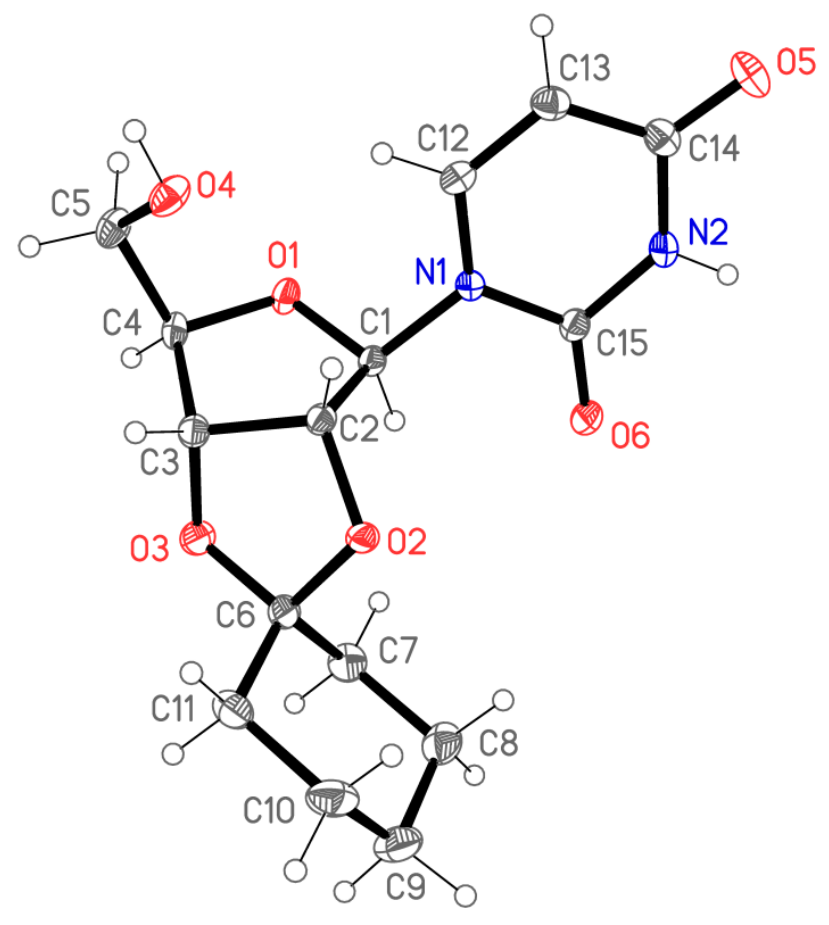

Figure S70. Structure of compound 1a in the crystal. Thermal ellipsoid plots (all at the $50 \%$ probability level).

Crystallization was performed by slow evaporation of benzene from a round-bottom flask.

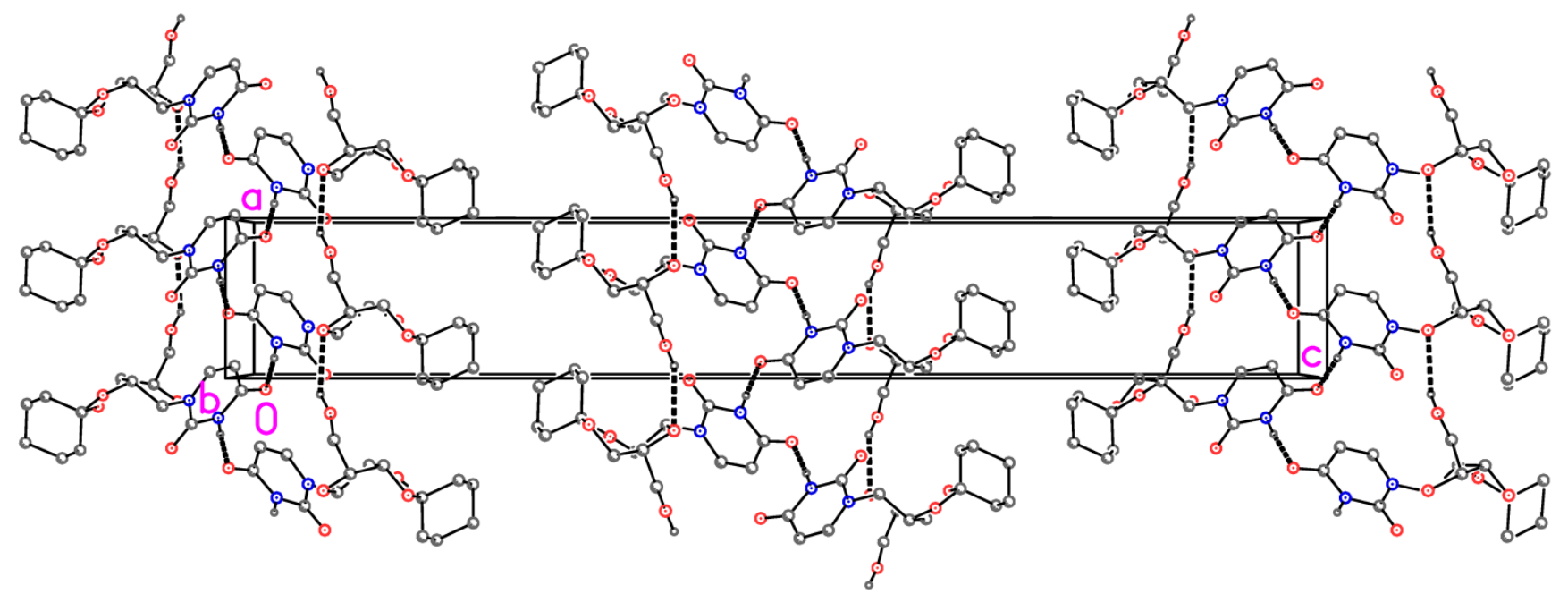

Figure S71. Packing diagram of compound 1a. Dashed lines indicate hydrogen bonds (for details see the deposited CIF file). The molecules associate to form corrugated ribbons parallel to the ac plane; the view direction is perpendicular to this plane. 
Table S2. Crystal Data and Structure Refinement.

\begin{tabular}{|c|c|c|}
\hline Identification code & \multicolumn{2}{|l|}{ kolbenz } \\
\hline Empirical formula & \multicolumn{2}{|l|}{$\mathrm{C}_{15} \mathrm{H}_{20} \mathrm{~N}_{2} \mathrm{O}_{6}$} \\
\hline Formula weight & \multicolumn{2}{|l|}{324.33} \\
\hline Temperature & \multicolumn{2}{|l|}{$100(2) \mathrm{K}$} \\
\hline Wavelength & \multicolumn{2}{|l|}{$1.54184 \AA$} \\
\hline Crystal system & \multicolumn{2}{|l|}{ Orthorhombic } \\
\hline Space group & \multicolumn{2}{|l|}{$P 212121$} \\
\hline \multirow[t]{3}{*}{ Unit cell dimensions } & $a=5.91240(8) \AA$ & $\alpha=90^{\circ}$ \\
\hline & $b=6.26542(6) \AA$ & $\beta=90^{\circ}$ \\
\hline & $c=40.7670(4) \AA$ & $Y=90^{\circ}$ \\
\hline Volume & \multicolumn{2}{|l|}{$1510.16(3) \AA^{3}$} \\
\hline Z & \multicolumn{2}{|l|}{4} \\
\hline Density (calculated) & \multicolumn{2}{|l|}{$1.427 \mathrm{Mg} / \mathrm{m}^{3}$} \\
\hline Absorption coefficient & \multicolumn{2}{|l|}{$0.935 \mathrm{~mm}^{-1}$} \\
\hline$F(000)$ & \multicolumn{2}{|l|}{688} \\
\hline Crystal size & \multicolumn{2}{|c|}{$0.15 \times 0.04 \times 0.02 \mathrm{~mm}^{3}$} \\
\hline Theta range for data collection & \multicolumn{2}{|l|}{2.167 to $77.476^{\circ}$} \\
\hline Index ranges & \multicolumn{2}{|c|}{$-6<=h<=7,-7<=k<=7,-51<=\mid<=51$} \\
\hline Reflections collected & \multicolumn{2}{|l|}{59504} \\
\hline Independent reflections & \multicolumn{2}{|c|}{$3181[R($ int $)=0.0425]$} \\
\hline Completeness to theta $=67.684^{\circ}$ & \multicolumn{2}{|l|}{$100.0 \%$} \\
\hline
\end{tabular}


Absorption correction

Max. and min. transmission

Refinement method

Data / restraints / parameters

Goodness-of-fit on $F^{2}$

Final $R$ indices [l>2sigma(I)]

$R$ indices (all data)

Absolute structure parameter

Largest diff. peak and hole
Semi-empirical from equivalents

1.00000 and 0.85681

Full-matrix least-squares on $\mathrm{F}^{2}$

$3181 / 0 / 216$

1.042

$\mathrm{R} 1=0.0248, \mathrm{wR} 2=0.0626$

$\mathrm{R} 1=0.0254, \mathrm{wR} 2=0.0630$

$-0.01(3)$

0.157 and -0.191 e..$^{-3}$ 
Table S3. Atomic Coordinates $\left(x 1^{4}\right)$ and Equivalent Isotropic Displacement Parameters $\left(\AA^{2} \times 10^{3}\right)$. U(eq) is Defined as one Third of the Trace of the Orthogonalized Uij Tensor.

\begin{tabular}{|c|c|c|c|c|}
\hline & $x$ & $y$ & $z$ & $\mathrm{U}(\mathrm{eq})$ \\
\hline$C(1)$ & $7222(2)$ & $3361(2)$ & $4021(1)$ & $13(1)$ \\
\hline$C(2)$ & $5552(2)$ & $2899(2)$ & $3739(1)$ & $13(1)$ \\
\hline$C(3)$ & $5446(2)$ & $5033(2)$ & $3565(1)$ & $15(1)$ \\
\hline$C(4)$ & $5873(3)$ & $6678(2)$ & $3833(1)$ & $15(1)$ \\
\hline$C(5)$ & $3713(3)$ & $7655(2)$ & $3959(1)$ & $18(1)$ \\
\hline$C(6)$ & $7603(3)$ & $2681(2)$ & $3257(1)$ & $16(1)$ \\
\hline$C(7)$ & $10111(3)$ & $2156(2)$ & $3261(1)$ & $20(1)$ \\
\hline$C(8)$ & 10528(3) & $-175(3)$ & $3169(1)$ & $25(1)$ \\
\hline $\mathrm{C}(9)$ & $9475(3)$ & $-708(3)$ & $2837(1)$ & $31(1)$ \\
\hline$C(10)$ & $6963(3)$ & $-166(3)$ & $2831(1)$ & $28(1)$ \\
\hline$C(11)$ & $6546(3)$ & $2168(3)$ & $2926(1)$ & $21(1)$ \\
\hline$C(12)$ & $4910(3)$ & $2467(2)$ & $4507(1)$ & $16(1)$ \\
\hline$C(13)$ & $4480(3)$ & $1325(2)$ & $4780(1)$ & $18(1)$ \\
\hline$C(14)$ & $6068(3)$ & $-276(2)$ & $4886(1)$ & $17(1)$ \\
\hline$C(15)$ & $8298(2)$ & $460(2)$ & $4388(1)$ & $14(1)$ \\
\hline$O(1)$ & $6987(2)$ & $5544(2)$ & $4100(1)$ & $16(1)$ \\
\hline $\mathrm{O}(2)$ & $6429(2)$ & $1450(2)$ & $3504(1)$ & $15(1)$ \\
\hline $\mathrm{O}(3)$ & $7324(2)$ & $4873(2)$ & $3345(1)$ & $19(1)$ \\
\hline
\end{tabular}




$\begin{array}{rrrrr}\mathrm{O}(4) & 2141(2) & 5982(2) & 4028(1) & 20(1) \\ \mathrm{O}(5) & 5966(2) & -1295(2) & 5144(1) & 24(1) \\ \mathrm{O}(6) & 9882(2) & -5(2) & 4211(1) & 20(1) \\ \mathrm{N}(1) & 6799(2) & 2099(2) & 4317(1) & 14(1) \\ \mathrm{N}(2) & 7851(2) & -615(2) & 4674(1) & 17(1)\end{array}$


Table S4. Bond Lengths $[\AA]$ and Angles $\left[^{\circ}\right]$.

\begin{tabular}{|c|c|c|c|}
\hline $\mathrm{C}(1)-\mathrm{O}(1)$ & $1.4123(16)$ & $C(6)-C(11)$ & $1.522(2)$ \\
\hline$C(1)-N(1)$ & $1.4661(17)$ & $C(7)-C(8)$ & $1.527(2)$ \\
\hline$C(1)-C(2)$ & $1.5417(19)$ & $C(8)-C(9)$ & $1.525(2)$ \\
\hline $\mathrm{C}(2)-\mathrm{O}(2)$ & $1.4179(16)$ & $C(9)-C(10)$ & $1.524(3)$ \\
\hline$C(2)-C(3)$ & $1.5148(19)$ & $C(10)-C(11)$ & $1.533(2)$ \\
\hline $\mathrm{C}(3)-\mathrm{O}(3)$ & $1.4324(17)$ & $C(12)-C(13)$ & $1.347(2)$ \\
\hline$C(3)-C(4)$ & $1.522(2)$ & $C(12)-N(1)$ & $1.3771(19)$ \\
\hline $\mathrm{C}(4)-\mathrm{O}(1)$ & $1.4562(16)$ & $C(13)-C(14)$ & $1.441(2)$ \\
\hline$C(4)-C(5)$ & $1.506(2)$ & $C(14)-O(5)$ & $1.2318(18)$ \\
\hline $\mathrm{C}(5)-\mathrm{O}(4)$ & $1.4287(18)$ & $C(14)-N(2)$ & $1.379(2)$ \\
\hline $\mathrm{C}(6)-\mathrm{O}(3)$ & $1.4286(17)$ & $C(15)-O(6)$ & $1.2180(18)$ \\
\hline $\mathrm{C}(6)-\mathrm{O}(2)$ & $1.4462(17)$ & $C(15)-N(2)$ & $1.3737(18)$ \\
\hline $\mathrm{C}(6)-\mathrm{C}(7)$ & $1.519(2)$ & $C(15)-N(1)$ & $1.3868(18)$ \\
\hline $\mathrm{O}(1)-\mathrm{C}(1)-\mathrm{N}(1)$ & $108.48(10)$ & $\mathrm{O}(3)-\mathrm{C}(3)-\mathrm{C}(2)$ & $101.53(11)$ \\
\hline$O(1)-C(1)-C(2)$ & $106.79(11)$ & $\mathrm{O}(3)-\mathrm{C}(3)-\mathrm{C}(4)$ & $111.68(12)$ \\
\hline$N(1)-C(1)-C(2)$ & $113.81(11)$ & $C(2)-C(3)-C(4)$ & $104.79(11)$ \\
\hline $\mathrm{O}(2)-\mathrm{C}(2)-\mathrm{C}(3)$ & $105.33(11)$ & $\mathrm{O}(1)-\mathrm{C}(4)-\mathrm{C}(5)$ & $109.12(11)$ \\
\hline $\mathrm{O}(2)-\mathrm{C}(2)-\mathrm{C}(1)$ & $112.89(12)$ & $\mathrm{O}(1)-\mathrm{C}(4)-\mathrm{C}(3)$ & $106.27(11)$ \\
\hline$C(3)-C(2)-C(1)$ & $102.05(11)$ & $C(5)-C(4)-C(3)$ & $112.25(12)$ \\
\hline
\end{tabular}




\begin{tabular}{llll}
$\mathrm{O}(4)-\mathrm{C}(5)-\mathrm{C}(4)$ & $108.69(11)$ & $\mathrm{O}(5)-\mathrm{C}(14)-\mathrm{N}(2)$ & $119.50(14)$ \\
$\mathrm{O}(3)-\mathrm{C}(6)-\mathrm{O}(2)$ & $106.44(11)$ & $\mathrm{O}(5)-\mathrm{C}(14)-\mathrm{C}(13)$ & $125.87(14)$ \\
$\mathrm{O}(3)-\mathrm{C}(6)-\mathrm{C}(7)$ & $108.58(12)$ & $\mathrm{N}(2)-\mathrm{C}(14)-\mathrm{C}(13)$ & $114.62(12)$ \\
$\mathrm{O}(2)-\mathrm{C}(6)-\mathrm{C}(7)$ & $110.30(12)$ & $\mathrm{O}(6)-\mathrm{C}(15)-\mathrm{N}(2)$ & $122.32(13)$ \\
$\mathrm{O}(3)-\mathrm{C}(6)-\mathrm{C}(11)$ & $112.15(12)$ & $\mathrm{O}(6)-\mathrm{C}(15)-\mathrm{N}(1)$ & $123.05(13)$ \\
$\mathrm{O}(2)-\mathrm{C}(6)-\mathrm{C}(11)$ & $107.93(12)$ & $\mathrm{N}(2)-\mathrm{C}(15)-\mathrm{N}(1)$ & $114.63(12)$ \\
$\mathrm{C}(7)-\mathrm{C}(6)-\mathrm{C}(11)$ & $111.33(13)$ & $\mathrm{C}(1)-\mathrm{O}(1)-\mathrm{C}(4)$ & $110.27(10)$ \\
$\mathrm{C}(6)-\mathrm{C}(7)-\mathrm{C}(8)$ & $111.23(13)$ & $\mathrm{C}(2)-\mathrm{O}(2)-\mathrm{C}(6)$ & $107.74(10)$ \\
$\mathrm{C}(9)-\mathrm{C}(8)-\mathrm{C}(7)$ & $111.17(14)$ & $\mathrm{C}(6)-\mathrm{O}(3)-\mathrm{C}(3)$ & $108.31(11)$ \\
$\mathrm{C}(10)-\mathrm{C}(9)-\mathrm{C}(8)$ & $111.34(13)$ & $\mathrm{C}(12)-\mathrm{N}(1)-\mathrm{C}(15)$ & $121.73(12)$ \\
$\mathrm{C}(9)-\mathrm{C}(10)-\mathrm{C}(11)$ & $111.43(15)$ & $\mathrm{C}(12)-\mathrm{N}(1)-\mathrm{C}(1)$ & $120.70(12)$ \\
$\mathrm{C}(6)-\mathrm{C}(11)-\mathrm{C}(10)$ & $111.08(13)$ & $\mathrm{C}(15)-\mathrm{N}(1)-\mathrm{C}(1)$ & $117.48(12)$ \\
$\mathrm{C}(13)-\mathrm{C}(12)-\mathrm{N}(1)$ & $121.80(14)$ & $\mathrm{C}(15)-\mathrm{N}(2)-\mathrm{C}(14)$ & $127.13(13)$ \\
$\mathrm{C}(12)-\mathrm{C}(13)-\mathrm{C}(14)$ & $119.73(14)$ & & \\
\hline
\end{tabular}

Table S5. Torsion Angles [ $\left.{ }^{\circ}\right]$.

\begin{tabular}{llll}
\hline $\mathrm{O}(1)-\mathrm{C}(1)-\mathrm{C}(2)-\mathrm{O}(2)$ & $142.32(11)$ & $\mathrm{C}(1)-\mathrm{C}(2)-\mathrm{C}(3)-\mathrm{C}(4)$ & $-29.78(14)$ \\
$\mathrm{N}(1)-\mathrm{C}(1)-\mathrm{C}(2)-\mathrm{O}(2)$ & $-98.01(13)$ & $\mathrm{O}(3)-\mathrm{C}(3)-\mathrm{C}(4)-\mathrm{O}(1)$ & $-88.75(13)$ \\
$\mathrm{O}(1)-\mathrm{C}(1)-\mathrm{C}(2)-\mathrm{C}(3)$ & $29.76(14)$ & $\mathrm{C}(2)-\mathrm{C}(3)-\mathrm{C}(4)-\mathrm{O}(1)$ & $20.36(14)$ \\
$\mathrm{N}(1)-\mathrm{C}(1)-\mathrm{C}(2)-\mathrm{C}(3)$ & $149.43(11)$ & $\mathrm{O}(3)-\mathrm{C}(3)-\mathrm{C}(4)-\mathrm{C}(5)$ & $152.03(12)$ \\
$\mathrm{O}(2)-\mathrm{C}(2)-\mathrm{C}(3)-\mathrm{O}(3)$ & $-31.53(14)$ & $\mathrm{C}(2)-\mathrm{C}(3)-\mathrm{C}(4)-\mathrm{C}(5)$ & $-98.86(13)$ \\
$\mathrm{C}(1)-\mathrm{C}(2)-\mathrm{C}(3)-\mathrm{O}(3)$ & $86.56(12)$ & $\mathrm{O}(1)-\mathrm{C}(4)-\mathrm{C}(5)-\mathrm{O}(4)$ & $-69.38(14)$ \\
$\mathrm{O}(2)-\mathrm{C}(2)-\mathrm{C}(3)-\mathrm{C}(4)$ & $-147.88(12)$ & $\mathrm{C}(3)-\mathrm{C}(4)-\mathrm{C}(5)-\mathrm{O}(4)$ & $48.15(16)$
\end{tabular}




\begin{tabular}{|c|c|c|c|}
\hline $\mathrm{O}(3)-\mathrm{C}(6)-\mathrm{C}(7)-\mathrm{C}(8)$ & $180.00(11)$ & $C(11)-C(6)-O(2)-C(2)$ & $-122.74(13)$ \\
\hline $\mathrm{O}(2)-\mathrm{C}(6)-\mathrm{C}(7)-\mathrm{C}(8)$ & $63.72(16)$ & $\mathrm{O}(2)-\mathrm{C}(6)-\mathrm{O}(3)-\mathrm{C}(3)$ & $-18.99(15)$ \\
\hline$C(11)-C(6)-C(7)-C(8)$ & $-56.07(17)$ & $\mathrm{C}(7)-\mathrm{C}(6)-\mathrm{O}(3)-\mathrm{C}(3)$ & $-137.73(12)$ \\
\hline$C(6)-C(7)-C(8)-C(9)$ & $55.67(17)$ & $C(11)-C(6)-O(3)-C(3)$ & $98.82(13)$ \\
\hline$C(7)-C(8)-C(9)-C(10)$ & $-55.03(19)$ & $C(2)-C(3)-O(3)-C(6)$ & $30.75(14)$ \\
\hline$C(8)-C(9)-C(10)-C(11)$ & $54.73(19)$ & $\mathrm{C}(4)-\mathrm{C}(3)-\mathrm{O}(3)-\mathrm{C}(6)$ & $141.95(12)$ \\
\hline $\mathrm{O}(3)-\mathrm{C}(6)-\mathrm{C}(11)-\mathrm{C}(10)$ & $177.42(13)$ & $C(13)-C(12)-N(1)-C(15)$ & $3.5(2)$ \\
\hline $\mathrm{O}(2)-\mathrm{C}(6)-\mathrm{C}(11)-\mathrm{C}(10)$ & $-65.66(16)$ & $C(13)-C(12)-N(1)-C(1)$ & $179.78(13)$ \\
\hline$C(7)-C(6)-C(11)-C(10)$ & $55.53(17)$ & $\mathrm{O}(6)-\mathrm{C}(15)-\mathrm{N}(1)-\mathrm{C}(12)$ & $174.14(14)$ \\
\hline$C(9)-C(10)-C(11)-C(6)$ & $-54.89(17)$ & $N(2)-C(15)-N(1)-C(12)$ & $-5.59(19)$ \\
\hline$N(1)-C(12)-C(13)-C(14)$ & $2.3(2)$ & $\mathrm{O}(6)-\mathrm{C}(15)-\mathrm{N}(1)-\mathrm{C}(1)$ & $-2.3(2)$ \\
\hline $\mathrm{C}(12)-\mathrm{C}(13)-\mathrm{C}(14)-\mathrm{O}(5)$ & $173.57(15)$ & $N(2)-C(15)-N(1)-C(1)$ & $177.99(12)$ \\
\hline$C(12)-C(13)-C(14)-N(2)$ & $-5.3(2)$ & $\mathrm{O}(1)-\mathrm{C}(1)-\mathrm{N}(1)-\mathrm{C}(12)$ & $49.33(17)$ \\
\hline$N(1)-C(1)-O(1)-C(4)$ & $-141.04(11)$ & $C(2)-C(1)-N(1)-C(12)$ & $-69.38(16)$ \\
\hline $\mathrm{C}(2)-\mathrm{C}(1)-\mathrm{O}(1)-\mathrm{C}(4)$ & $-17.99(15)$ & $O(1)-C(1)-N(1)-C(15)$ & $-134.21(12)$ \\
\hline $\mathrm{C}(5)-\mathrm{C}(4)-\mathrm{O}(1)-\mathrm{C}(1)$ & $119.86(12)$ & $C(2)-C(1)-N(1)-C(15)$ & $107.08(14)$ \\
\hline$C(3)-C(4)-O(1)-C(1)$ & $-1.39(15)$ & $\mathrm{O}(6)-\mathrm{C}(15)-\mathrm{N}(2)-\mathrm{C}(14)$ & $-177.50(14)$ \\
\hline $\mathrm{C}(3)-\mathrm{C}(2)-\mathrm{O}(2)-\mathrm{C}(6)$ & $21.04(15)$ & $N(1)-C(15)-N(2)-C(14)$ & $2.2(2)$ \\
\hline $\mathrm{C}(1)-\mathrm{C}(2)-\mathrm{O}(2)-\mathrm{C}(6)$ & $-89.50(13)$ & $\mathrm{O}(5)-\mathrm{C}(14)-\mathrm{N}(2)-\mathrm{C}(15)$ & $-175.89(14)$ \\
\hline $\mathrm{O}(3)-\mathrm{C}(6)-\mathrm{O}(2)-\mathrm{C}(2)$ & $-2.18(15)$ & $\mathrm{C}(13)-\mathrm{C}(14)-\mathrm{N}(2)-\mathrm{C}(15)$ & $3.1(2)$ \\
\hline $\mathrm{C}(7)-\mathrm{C}(6)-\mathrm{O}(2)-\mathrm{C}(2)$ & $115.44(13)$ & & \\
\hline
\end{tabular}


Table S6. Hydrogen Bonds [ $\AA$ and $\left.{ }^{\circ}\right]$.

\begin{tabular}{lcccc}
\hline $\mathrm{D}-\mathrm{H} \ldots \mathrm{A}$ & $\mathrm{d}(\mathrm{D}-\mathrm{H})$ & $\mathrm{d}(\mathrm{H} \ldots \mathrm{A})$ & $\mathrm{d}(\mathrm{D} \ldots \mathrm{A})$ & $<(\mathrm{DHA})$ \\
\hline $\mathrm{O}(4)-\mathrm{H}(04) \ldots \mathrm{O}(1) \# 1$ & $0.85(3)$ & $2.42(2)$ & $3.0735(16)$ & $134(2)$ \\
$\mathrm{O}(4)-\mathrm{H}(04) \ldots \mathrm{O}(6) \# 2$ & $0.85(3)$ & $2.30(3)$ & $2.9434(15)$ & $133(2)$ \\
$\mathrm{N}(2)-\mathrm{H}(02) \ldots \mathrm{O}(5) \# 3$ & $0.89(2)$ & $1.88(2)$ & $2.7727(17)$ & $176(2)$ \\
$\mathrm{C}(4)-\mathrm{H}(4) \ldots \mathrm{O}(2) \# 4$ & 1.00 & 2.49 & $3.2932(17)$ & 136.6 \\
$\mathrm{C}(12)-\mathrm{H}(12) \ldots \mathrm{O}(4)$ & 0.95 & 2.50 & $3.3679(18)$ & 152.4 \\
\end{tabular}

Symmetry transformations used to generate equivalent atoms:

$\# 1 \mathrm{x}-1, \mathrm{y}, \mathrm{z}$

$\# 2 x-1, y+1, z$

$\# 3 x+1 / 2,-y-1 / 2,-z+1$

$\# 4 x, y+1, z$ 


\section{X-Ray Data for Compound 1b}

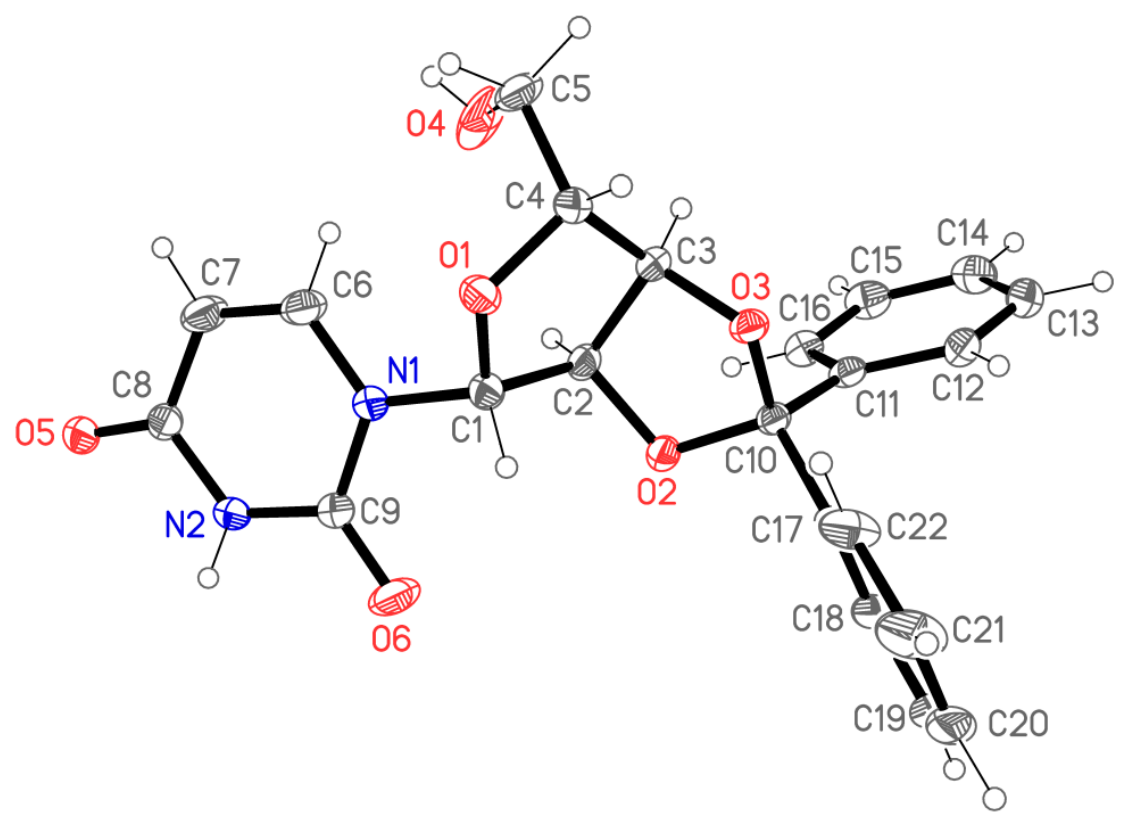

Figure S72. Structure of compound $\mathbf{1 b}$ in the crystal. Thermal ellipsoid plots (all at the $50 \%$ probability level).

Crystallization was performed by slow evaporation of benzene from a round-bottom flask.

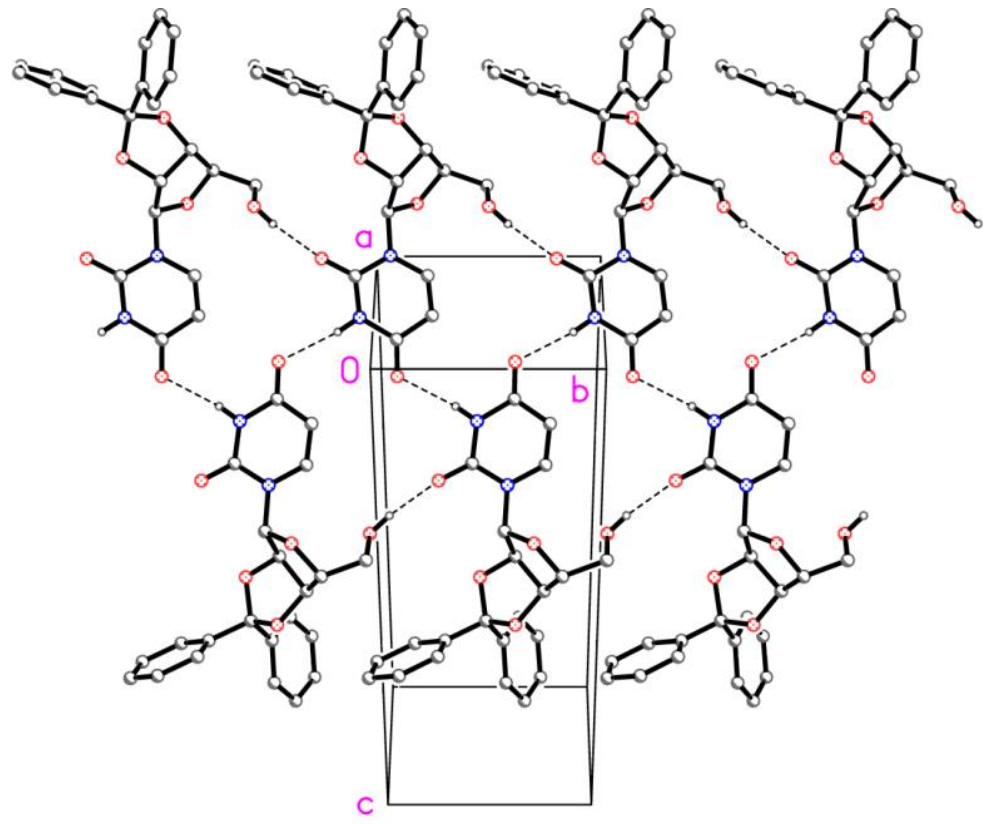

Figure S73. Packing diagram of compound 1b. Dashed lines indicate hydrogen bonds (for details see the deposited CIF file). The molecules associate to form broad ribbons parallel to (104); the view direction is perpendicular to this plane. 
Table S7. Crystal Data and Structure Refinement.

\begin{tabular}{|c|c|c|}
\hline Identification code & \multicolumn{2}{|l|}{ koell5 } \\
\hline Empirical formula & \multicolumn{2}{|l|}{$\mathrm{C}_{22} \mathrm{H}_{20} \mathrm{~N}_{2} \mathrm{O}_{6}$} \\
\hline Formula weight & \multicolumn{2}{|l|}{408.40} \\
\hline Temperature & \multicolumn{2}{|l|}{$100(2) \mathrm{K}$} \\
\hline Wavelength & \multicolumn{2}{|l|}{$1.54184 \AA$} \\
\hline Crystal system & \multicolumn{2}{|l|}{ Monoclinic } \\
\hline Space group & \multicolumn{2}{|l|}{$\mathrm{P} 21$} \\
\hline \multirow[t]{3}{*}{ Unit cell dimensions } & $\mathrm{a}=5.99677(7) \AA$ & $\alpha=90^{\circ}$ \\
\hline & $\mathrm{b}=7.61343(8) \AA$ & $\beta=92.4786(10)^{\circ}$ \\
\hline & $c=20.4686(2) \AA$ & $Y=90^{\circ}$ \\
\hline Volume & \multicolumn{2}{|l|}{$933.641(17) \AA^{3}$} \\
\hline Z & \multicolumn{2}{|l|}{2} \\
\hline Density (calculated) & \multicolumn{2}{|l|}{$1.453 \mathrm{Mg} / \mathrm{m}^{3}$} \\
\hline Absorption coefficient & \multicolumn{2}{|l|}{$0.891 \mathrm{~mm}^{-1}$} \\
\hline$F(000)$ & \multicolumn{2}{|l|}{428} \\
\hline Crystal size & \multicolumn{2}{|c|}{$0.15 \times 0.12 \times 0.04 \mathrm{~mm}^{3}$} \\
\hline Theta range for data collection & \multicolumn{2}{|l|}{4.324 to $78.029^{\circ}$} \\
\hline Index ranges & \multicolumn{2}{|c|}{$-7<=\mathrm{h}<=7,-9<=\mathrm{k}<=9,-25<=\mid<=25$} \\
\hline Reflections collected & \multicolumn{2}{|l|}{58257} \\
\hline Independent reflections & \multicolumn{2}{|c|}{$3967[R($ int $)=0.0444]$} \\
\hline Completeness to theta $=75.000^{\circ}$ & \multicolumn{2}{|l|}{$100.0 \%$} \\
\hline
\end{tabular}


Absorption correction

Max. and min. transmission

Refinement method

Data / restraints / parameters

Goodness-of-fit on F2

Final $R$ indices [l>2sigma(I)]

$\mathrm{R}$ indices (all data)

Absolute structure parameter

Largest diff. peak and hole
Semi-empirical from equivalents

1.00000 and 0.68593

Full-matrix least-squares on $\mathrm{F}^{2}$

3967 / 1 / 279

1.028

$R 1=0.0338, w R 2=0.0880$

$\mathrm{R} 1=0.0340, \mathrm{wR} 2=0.0882$

$0.01(4)$

0.311 and -0.252 e. $\AA^{-3}$ 
Table S8. Atomic Coordinates $\left(x 1^{4}\right)$ and Equivalent Isotropic Displacement Parameters $\left(\AA^{2} \times 10^{3}\right)$. U(eq) is Defined as one Third of the Trace of the Orthogonalized Uij Tensor.

\begin{tabular}{|c|c|c|c|c|}
\hline & $x$ & $y$ & $z$ & $\mathrm{U}(\mathrm{eq})$ \\
\hline$C(1)$ & $3059(3)$ & $5682(3)$ & $1529(1)$ & $19(1)$ \\
\hline$C(2)$ & $3784(3)$ & $6135(3)$ & $2246(1)$ & $19(1)$ \\
\hline$C(3)$ & 1938(3) & $7342(3)$ & $2467(1)$ & $21(1)$ \\
\hline$C(4)$ & $806(4)$ & $8077(3)$ & $1849(1)$ & $27(1)$ \\
\hline$C(5)$ & $1525(7)$ & $9928(3)$ & $1709(1)$ & $52(1)$ \\
\hline$C(6)$ & $5397(4)$ & 7392(3) & $787(1)$ & $29(1)$ \\
\hline $\mathrm{C}(7)$ & $7074(4)$ & $7586(3)$ & $379(1)$ & $30(1)$ \\
\hline$C(8)$ & $8413(3)$ & $6096(3)$ & $216(1)$ & $20(1)$ \\
\hline $\mathrm{C}(9)$ & $6096(4)$ & $4318(3)$ & $935(1)$ & $25(1)$ \\
\hline$C(10)$ & $1905(3)$ & $4894(2)$ & $3095(1)$ & $16(1)$ \\
\hline$C(11)$ & $2729(3)$ & $5641(2)$ & $3757(1)$ & $19(1)$ \\
\hline$C(12)$ & $1217(3)$ & $5754(3)$ & $4255(1)$ & $23(1)$ \\
\hline$C(13)$ & $1814(4)$ & $6570(3)$ & $4844(1)$ & $27(1)$ \\
\hline$C(14)$ & $3934(4)$ & $7290(3)$ & $4941(1)$ & $29(1)$ \\
\hline$C(15)$ & $5456(4)$ & $7154(3)$ & $4455(1)$ & $27(1)$ \\
\hline$C(16)$ & $4868(3)$ & $6315(3)$ & $3864(1)$ & $22(1)$ \\
\hline$C(17)$ & $690(3)$ & $3170(2)$ & $3173(1)$ & $19(1)$ \\
\hline$C(18)$ & $1748(4)$ & 1848(3) & $3540(1)$ & $26(1)$ \\
\hline
\end{tabular}




$\begin{array}{lrrrr}\mathrm{C}(19) & 676(5) & 260(3) & 3629(1) & 38(1) \\ \mathrm{C}(20) & -1435(5) & -5(3) & 3359(2) & 49(1) \\ \mathrm{C}(21) & -2492(4) & 1299(4) & 2994(2) & 49(1) \\ \mathrm{C}(22) & -1425(3) & 2899(3) & 2900(1) & 31(1) \\ \mathrm{O}(1) & 1384(2) & 6889(2) & 1330(1) & 22(1) \\ \mathrm{O}(2) & 3711(2) & 4643(2) & 2665(1) & 19(1) \\ \mathrm{O}(3) & 477(2) & 6137(2) & 2773(1) & 19(1) \\ \mathrm{O}(4) & 3865(5) & 10017(3) & 1793(1) & 61(1) \\ \mathrm{O}(5) & 10002(2) & 6134(2) & -143(1) & 23(1) \\ \mathrm{O}(6) & 5722(4) & 2905(2) & 1188(1) & 54(1) \\ \mathrm{N}(1) & 4890(3) & 5804(2) & 1067(1) & 20(1) \\ \mathrm{N}(2) & 7756(3) & 4525(2) & 498(1) & 22(1)\end{array}$

Table S9. Bond Lengths $\left[\AA ̊ \AA\right.$ and Angles $\left[^{\circ}\right]$.

\begin{tabular}{llll}
\hline $\mathrm{C}(1)-\mathrm{O}(1)$ & $1.409(2)$ & $\mathrm{C}(5)-\mathrm{O}(4)$ & $1.408(5)$ \\
$\mathrm{C}(1)-\mathrm{N}(1)$ & $1.483(2)$ & $\mathrm{C}(6)-\mathrm{C}(7)$ & $1.342(3)$ \\
$\mathrm{C}(1)-\mathrm{C}(2)$ & $1.551(2)$ & $\mathrm{C}(6)-\mathrm{N}(1)$ & $1.378(3)$ \\
$\mathrm{C}(2)-\mathrm{O}(2)$ & $1.425(2)$ & $\mathrm{C}(7)-\mathrm{C}(8)$ & $1.437(3)$ \\
$\mathrm{C}(2)-\mathrm{C}(3)$ & $1.523(3)$ & $\mathrm{C}(8)-\mathrm{O}(5)$ & $1.229(2)$ \\
$\mathrm{C}(3)-\mathrm{O}(3)$ & $1.431(2)$ & $\mathrm{C}(8)-\mathrm{N}(2)$ & $1.392(2)$ \\
$\mathrm{C}(3)-\mathrm{C}(4)$ & $1.516(3)$ & $\mathrm{C}(9)-\mathrm{O}(6)$ & $1.220(3)$ \\
$\mathrm{C}(4)-\mathrm{O}(1)$ & $1.450(2)$ & $\mathrm{C}(9)-\mathrm{N}(2)$ & $1.376(3)$ \\
$\mathrm{C}(4)-\mathrm{C}(5)$ & $1.505(3)$ & $\mathrm{C}(9)-\mathrm{N}(1)$ & $1.376(3)$ \\
& & $\mathrm{S} 89$
\end{tabular}




\begin{tabular}{|c|c|c|c|}
\hline$C(10)-O(3)$ & $1.420(2)$ & $C(14)-C(15)$ & $1.382(3)$ \\
\hline $\mathrm{C}(10)-\mathrm{O}(2)$ & $1.437(2)$ & $C(15)-C(16)$ & $1.400(3)$ \\
\hline$C(10)-C(17)$ & $1.513(3)$ & $C(17)-C(22)$ & $1.379(3)$ \\
\hline$C(10)-C(11)$ & $1.531(2)$ & $C(17)-C(18)$ & $1.392(3)$ \\
\hline$C(11)-C(16)$ & $1.390(3)$ & $C(18)-C(19)$ & $1.385(3)$ \\
\hline$C(11)-C(12)$ & $1.397(3)$ & $C(19)-C(20)$ & $1.374(5)$ \\
\hline$C(12)-C(13)$ & $1.390(3)$ & $C(20)-C(21)$ & $1.380(5)$ \\
\hline$C(13)-C(14)$ & $1.391(3)$ & $\mathrm{C}(21)-\mathrm{C}(22)$ & $1.393(3)$ \\
\hline $\mathrm{O}(1)-\mathrm{C}(1)-\mathrm{N}(1)$ & $108.18(14)$ & $C(6)-C(7)-C(8)$ & $119.93(19)$ \\
\hline $\mathrm{O}(1)-\mathrm{C}(1)-\mathrm{C}(2)$ & $107.25(15)$ & $\mathrm{O}(5)-\mathrm{C}(8)-\mathrm{N}(2)$ & $120.57(18)$ \\
\hline$N(1)-C(1)-C(2)$ & $113.86(14)$ & $\mathrm{O}(5)-\mathrm{C}(8)-\mathrm{C}(7)$ & $125.14(19)$ \\
\hline $\mathrm{O}(2)-\mathrm{C}(2)-\mathrm{C}(3)$ & $105.10(14)$ & $\mathrm{N}(2)-\mathrm{C}(8)-\mathrm{C}(7)$ & $114.28(16)$ \\
\hline $\mathrm{O}(2)-\mathrm{C}(2)-\mathrm{C}(1)$ & $112.19(15)$ & $\mathrm{O}(6)-\mathrm{C}(9)-\mathrm{N}(2)$ & $121.95(19)$ \\
\hline$C(3)-C(2)-C(1)$ & $103.75(15)$ & $\mathrm{O}(6)-\mathrm{C}(9)-\mathrm{N}(1)$ & $122.25(19)$ \\
\hline $\mathrm{O}(3)-\mathrm{C}(3)-\mathrm{C}(4)$ & $109.93(16)$ & $N(2)-C(9)-N(1)$ & $115.80(17)$ \\
\hline $\mathrm{O}(3)-\mathrm{C}(3)-\mathrm{C}(2)$ & $102.11(15)$ & $\mathrm{O}(3)-\mathrm{C}(10)-\mathrm{O}(2)$ & $105.04(13)$ \\
\hline$C(4)-C(3)-C(2)$ & $106.24(15)$ & $\mathrm{O}(3)-\mathrm{C}(10)-\mathrm{C}(17)$ & $110.09(14)$ \\
\hline$O(1)-C(4)-C(5)$ & $111.41(17)$ & $\mathrm{O}(2)-\mathrm{C}(10)-\mathrm{C}(17)$ & $109.15(14)$ \\
\hline$O(1)-C(4)-C(3)$ & $105.63(16)$ & $\mathrm{O}(3)-\mathrm{C}(10)-\mathrm{C}(11)$ & $109.16(14)$ \\
\hline$C(5)-C(4)-C(3)$ & $112.5(2)$ & $\mathrm{O}(2)-\mathrm{C}(10)-\mathrm{C}(11)$ & $111.59(14)$ \\
\hline$O(4)-C(5)-C(4)$ & 108.3(2) & $C(17)-C(10)-C(11)$ & $111.60(15)$ \\
\hline$C(7)-C(6)-N(1)$ & $122.68(19)$ & $C(16)-C(11)-C(12)$ & $119.05(17)$ \\
\hline
\end{tabular}




$\begin{array}{llll}C(16)-C(11)-C(10) & 122.52(17) & C(20)-C(19)-C(18) & 120.0(2) \\ C(12)-C(11)-C(10) & 118.29(16) & C(19)-C(20)-C(21) & 120.4(2) \\ C(13)-C(12)-C(11) & 120.69(18) & C(20)-C(21)-C(22) & 120.0(2) \\ C(12)-C(13)-C(14) & 119.92(19) & C(17)-C(22)-C(21) & 119.6(2) \\ C(15)-C(14)-C(13) & 119.79(18) & C(1)-O(1)-C(4) & 112.59(14) \\ C(14)-C(15)-C(16) & 120.42(19) & C(2)-O(2)-C(10) & 107.82(13) \\ C(11)-C(16)-C(15) & 120.08(18) & C(10)-O(3)-C(3) & 105.20(13) \\ C(22)-C(17)-C(18) & 120.01(19) & C(9)-N(1)-C(6) & 120.80(16) \\ C(22)-C(17)-C(10) & 121.72(18) & C(9)-N(1)-C(1) & 118.94(15) \\ C(18)-C(17)-C(10) & 118.27(17) & C(6)-N(1)-C(1) & 120.24(16) \\ C(19)-C(18)-C(17) & 119.9(2) & C(9)-N(2)-C(8) & 126.36(17)\end{array}$


Table S10. Torsion Angles $\left[{ }^{\circ}\right]$.

\begin{tabular}{|c|c|c|c|}
\hline $\mathrm{O}(1)-\mathrm{C}(1)-\mathrm{C}(2)-\mathrm{O}(2)$ & $127.85(15)$ & $O(2)-C(10)-C(11)-C(12)$ & $-172.18(16)$ \\
\hline$N(1)-C(1)-C(2)-O(2)$ & $-112.50(17)$ & $C(17)-C(10)-C(11)-C(12)$ & $-49.8(2)$ \\
\hline$O(1)-C(1)-C(2)-C(3)$ & $14.93(19)$ & $C(16)-C(11)-C(12)-C(13)$ & $1.8(3)$ \\
\hline$N(1)-C(1)-C(2)-C(3)$ & $134.57(16)$ & $C(10)-C(11)-C(12)-C(13)$ & $-173.98(17)$ \\
\hline $\mathrm{O}(2)-\mathrm{C}(2)-\mathrm{C}(3)-\mathrm{O}(3)$ & $-23.76(17)$ & $C(11)-C(12)-C(13)-C(14)$ & $0.2(3)$ \\
\hline$C(1)-C(2)-C(3)-O(3)$ & $94.19(16)$ & $C(12)-C(13)-C(14)-C(15)$ & $-1.5(3)$ \\
\hline $\mathrm{O}(2)-\mathrm{C}(2)-\mathrm{C}(3)-\mathrm{C}(4)$ & $-138.95(16)$ & $C(13)-C(14)-C(15)-C(16)$ & $0.8(3)$ \\
\hline$C(1)-C(2)-C(3)-C(4)$ & $-21.0(2)$ & $C(12)-C(11)-C(16)-C(15)$ & $-2.5(3)$ \\
\hline $\mathrm{O}(3)-\mathrm{C}(3)-\mathrm{C}(4)-\mathrm{O}(1)$ & $-89.97(18)$ & $C(10)-C(11)-C(16)-C(15)$ & $173.07(18)$ \\
\hline $\mathrm{C}(2)-\mathrm{C}(3)-\mathrm{C}(4)-\mathrm{O}(1)$ & $19.8(2)$ & $C(14)-C(15)-C(16)-C(11)$ & $1.2(3)$ \\
\hline $\mathrm{O}(3)-\mathrm{C}(3)-\mathrm{C}(4)-\mathrm{C}(5)$ & $148.3(2)$ & $\mathrm{O}(3)-\mathrm{C}(10)-\mathrm{C}(17)-\mathrm{C}(22)$ & $5.6(2)$ \\
\hline$C(2)-C(3)-C(4)-C(5)$ & $-102.0(2)$ & $\mathrm{O}(2)-\mathrm{C}(10)-\mathrm{C}(17)-\mathrm{C}(22)$ & $-109.2(2)$ \\
\hline $\mathrm{O}(1)-\mathrm{C}(4)-\mathrm{C}(5)-\mathrm{O}(4)$ & $-72.6(2)$ & $C(11)-C(10)-C(17)-C(22)$ & 127.0(2) \\
\hline $\mathrm{C}(3)-\mathrm{C}(4)-\mathrm{C}(5)-\mathrm{O}(4)$ & $45.8(2)$ & $\mathrm{O}(3)-\mathrm{C}(10)-\mathrm{C}(17)-\mathrm{C}(18)$ & $-173.34(16)$ \\
\hline$N(1)-C(6)-C(7)-C(8)$ & $0.4(4)$ & $\mathrm{O}(2)-\mathrm{C}(10)-\mathrm{C}(17)-\mathrm{C}(18)$ & $71.9(2)$ \\
\hline $\mathrm{C}(6)-\mathrm{C}(7)-\mathrm{C}(8)-\mathrm{O}(5)$ & $-178.9(2)$ & $C(11)-C(10)-C(17)-C(18)$ & $-52.0(2)$ \\
\hline$C(6)-C(7)-C(8)-N(2)$ & $1.8(3)$ & $C(22)-C(17)-C(18)-C(19)$ & $0.2(3)$ \\
\hline $\mathrm{O}(3)-\mathrm{C}(10)-\mathrm{C}(11)-\mathrm{C}(16)$ & $-103.4(2)$ & $C(10)-C(17)-C(18)-C(19)$ & $179.15(17)$ \\
\hline $\mathrm{O}(2)-\mathrm{C}(10)-\mathrm{C}(11)-\mathrm{C}(16)$ & $12.2(2)$ & $C(17)-C(18)-C(19)-C(20)$ & $-0.4(3)$ \\
\hline$C(17)-C(10)-C(11)-C(16)$ & $134.65(18)$ & $C(18)-C(19)-C(20)-C(21)$ & $0.5(4)$ \\
\hline$O(3)-C(10)-C(11)-C(12)$ & 72.2(2) & $C(19)-C(20)-C(21)-C(22)$ & $-0.3(4)$ \\
\hline
\end{tabular}




$\begin{array}{lccc}\mathrm{C}(18)-\mathrm{C}(17)-\mathrm{C}(22)-\mathrm{C}(21) & 0.0(3) & \mathrm{C}(2)-\mathrm{C}(3)-\mathrm{O}(3)-\mathrm{C}(10) & 37.26(17) \\ \mathrm{C}(10)-\mathrm{C}(17)-\mathrm{C}(22)-\mathrm{C}(21) & -179.0(2) & \mathrm{O}(6)-\mathrm{C}(9)-\mathrm{N}(1)-\mathrm{C}(6) & 178.1(2) \\ \mathrm{C}(20)-\mathrm{C}(21)-\mathrm{C}(22)-\mathrm{C}(17) & 0.1(4) & \mathrm{N}(2)-\mathrm{C}(9)-\mathrm{N}(1)-\mathrm{C}(6) & -1.9(3) \\ \mathrm{N}(1)-\mathrm{C}(1)-\mathrm{O}(1)-\mathrm{C}(4) & -126.05(16) & \mathrm{O}(6)-\mathrm{C}(9)-\mathrm{N}(1)-\mathrm{C}(1) & -0.5(3) \\ \mathrm{C}(2)-\mathrm{C}(1)-\mathrm{O}(1)-\mathrm{C}(4) & -2.8(2) & \mathrm{N}(2)-\mathrm{C}(9)-\mathrm{N}(1)-\mathrm{C}(1) & 179.47(16) \\ \mathrm{C}(5)-\mathrm{C}(4)-\mathrm{O}(1)-\mathrm{C}(1) & 111.8(2) & \mathrm{C}(7)-\mathrm{C}(6)-\mathrm{N}(1)-\mathrm{C}(9) & -0.4(3) \\ \mathrm{C}(3)-\mathrm{C}(4)-\mathrm{O}(1)-\mathrm{C}(1) & -10.6(2) & \mathrm{C}(7)-\mathrm{C}(6)-\mathrm{N}(1)-\mathrm{C}(1) & 178.2(2) \\ \mathrm{C}(3)-\mathrm{C}(2)-\mathrm{O}(2)-\mathrm{C}(10) & 1.82(17) & \mathrm{O}(1)-\mathrm{C}(1)-\mathrm{N}(1)-\mathrm{C}(9) & -147.66(17) \\ \mathrm{C}(1)-\mathrm{C}(2)-\mathrm{O}(2)-\mathrm{C}(10) & -110.27(16) & \mathrm{C}(2)-\mathrm{C}(1)-\mathrm{N}(1)-\mathrm{C}(9) & 93.2(2) \\ \mathrm{O}(3)-\mathrm{C}(10)-\mathrm{O}(2)-\mathrm{C}(2) & 21.25(17) & \mathrm{O}(1)-\mathrm{C}(1)-\mathrm{N}(1)-\mathrm{C}(6) & 33.7(2) \\ \mathrm{C}(17)-\mathrm{C}(10)-\mathrm{O}(2)-\mathrm{C}(2) & 139.28(15) & \mathrm{C}(2)-\mathrm{C}(1)-\mathrm{N}(1)-\mathrm{C}(6) & -85.4(2) \\ \mathrm{C}(11)-\mathrm{C}(10)-\mathrm{O}(2)-\mathrm{C}(2) & -96.90(16) & \mathrm{O}(6)-\mathrm{C}(9)-\mathrm{N}(2)-\mathrm{C}(8) & -175.4(2) \\ \mathrm{O}(2)-\mathrm{C}(10)-\mathrm{O}(3)-\mathrm{C}(3) & -37.22(17) & \mathrm{N}(1)-\mathrm{C}(9)-\mathrm{N}(2)-\mathrm{C}(8) & 4.6(3) \\ \mathrm{C}(17)-\mathrm{C}(10)-\mathrm{O}(3)-\mathrm{C}(3) & -154.61(15) & \mathrm{O}(5)-\mathrm{C}(8)-\mathrm{N}(2)-\mathrm{C}(9) & 176.16(18) \\ \mathrm{C}(11)-\mathrm{C}(10)-\mathrm{O}(3)-\mathrm{C}(3) & 82.56(16) & \mathrm{C}(7)-\mathrm{C}(8)-\mathrm{N}(2)-\mathrm{C}(9) & -4.5(3) \\ \mathrm{C}(4)-\mathrm{C}(3)-\mathrm{O}(3)-\mathrm{C}(10) & 149.72(15) & & \\ & & & \\ & & \end{array}$

Table S11. Hydrogen Bonds [ $\AA$ and ${ }^{\circ}$ ].

\begin{tabular}{lcccc}
\hline D-H...A & $d(D-H)$ & $d(H \ldots A)$ & $d(D \ldots A)$ & $<(D H A)$ \\
\hline $\mathrm{O}(4)-\mathrm{H}(04) \ldots \mathrm{O}(6) \# 1$ & $0.85(6)$ & $1.94(7)$ & $2.779(3)$ & $169(6)$ \\
$\mathrm{N}(2)-\mathrm{H}(02) \ldots \mathrm{O}(5) \# 2$ & $0.88(4)$ & $2.14(4)$ & $3.014(2)$ & $168(3)$
\end{tabular}




$\begin{array}{lllll}\mathrm{C}(5)-\mathrm{H}(5 \mathrm{~A}) \ldots \mathrm{O}(5) \# 3 & 0.99 & 2.43 & 3.421(3) & 178.1 \\ \mathrm{C}(6)-\mathrm{H}(6) \ldots \mathrm{O}(4) & 0.95 & 2.28 & 3.040(3) & 136.5 \\ \mathrm{C}(7)-\mathrm{H}(7) \ldots \mathrm{O}(5) \# 4 & 0.95 & 2.43 & 3.268(3) & 146.6 \\ \mathrm{C}(21)-\mathrm{H}(21) \ldots \mathrm{O}(4) \# 5 & 0.95 & 2.55 & 3.363(4) & 144.3 \\ \mathrm{C}(22)-\mathrm{H}(22) \ldots \mathrm{O}(2) \# 6 & 0.95 & 2.56 & 3.222(2) & 126.5\end{array}$

Symmetry transformations used to generate equivalent atoms:

$\# 1 \mathrm{x}, \mathrm{y}+1, \mathrm{z}$

$\# 2-x+2, y-1 / 2,-z$

$\# 3-x+1, y+1 / 2,-z$

$\# 4-x+2, y+1 / 2,-z$

$\# 5 x-1, y-1, z$

$\# 6 x-1, y, z$ 


\section{X-Ray Data for Compound 11}

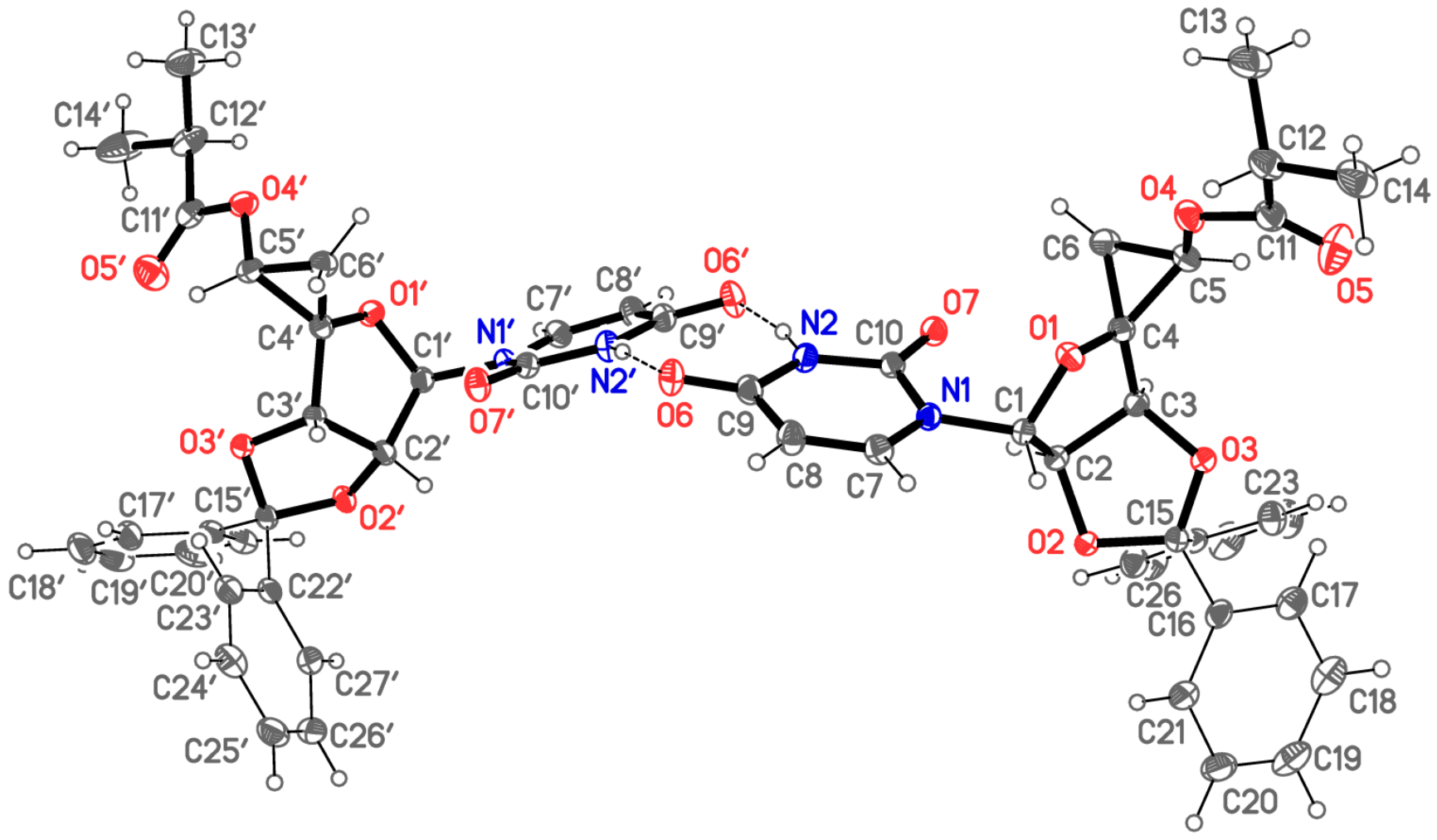

Figure S74. Structure of compound 11 in the crystal (two independent molecules; dashed lines indicate hydrogen bonds). Thermal ellipsoid plots (all at the 50\% probability level).

Crystallization was performed by slow evaporation of acetonitrile/water 70:30 from a round bottom flask.

Table S12. Crystal Data and Structure Refinement.

Identification code

Empirical formula

Formula weight

Temperature

Wavelength

Crystal system

Space group

Unit cell dimensions knotri

$\mathrm{C}_{27} \mathrm{H}_{26} \mathrm{~N}_{2} \mathrm{O}_{7}$

490.50

100(2) K

$1.54184 \AA$

Orthorhombic

P212121

$$
\begin{array}{ll}
a=10.75286(7) \AA & \alpha=90^{\circ} \\
b=10.83630(7) \AA & \beta=90^{\circ}
\end{array}
$$


Volume

Z

Density (calculated)

Absorption coefficient

$\mathrm{F}(000)$

Crystal size

Theta range for data collection

Index ranges

Reflections collected

Independent reflections

Completeness to theta $=77.000^{\circ}$

Absorption correction

Max. and min. transmission

Refinement method

Data / restraints / parameters

Goodness-of-fit on $\mathrm{F}^{2}$

Final $R$ indices [l>2sigma(I)]

$R$ indices (all data)

Absolute structure parameter

Extinction coefficient

Largest diff. peak and hole
$\mathrm{C}=41.1130(3) \AA \quad \mathrm{Y}=90^{\circ}$

4790.54(6) $\AA^{3}$

8

$1.360 \mathrm{Mg} / \mathrm{m}^{3}$

$0.821 \mathrm{~mm}^{-1}$

2064

$0.15 \times 0.10 \times 0.10 \mathrm{~mm}^{3}$

4.219 to $77.815^{\circ}$

$-13<=h<=13,-13<=k<=13,-51<=\mid<=52$

217865

$10099[R$ (int) $=0.0434]$

$99.6 \%$

Semi-empirical from equivalents

1.00000 and 0.84954

Full-matrix least-squares on $\mathrm{F}^{2}$

10099 / 0 / 662

1.021

$\mathrm{R} 1=0.0238, \mathrm{wR} 2=0.0603$

$R 1=0.0242, w R 2=0.0605$

$-0.018(19)$

$0.00029(4)$

0.216 and -0.163 e. $\AA^{-3}$ 
Table S13. Atomic Coordinates $\left(x 1^{4}\right)$ and Equivalent Isotropic Displacement Parameters $\left(\AA^{2} \times 10^{3}\right) . U(e q)$ is Defined as one Third of the Trace of the Orthogonalized Uij Tensor.

\begin{tabular}{|c|c|c|c|c|}
\hline & $x$ & $y$ & $z$ & $\mathrm{U}(\mathrm{eq})$ \\
\hline$C(1)$ & $6349(1)$ & $736(1)$ & $1282(1)$ & $16(1)$ \\
\hline$C(2)$ & $7145(1)$ & $1681(1)$ & $1090(1)$ & $16(1)$ \\
\hline$C(3)$ & $8394(1)$ & $1661(1)$ & $1271(1)$ & $16(1)$ \\
\hline$C(4)$ & $8042(1)$ & $1226(1)$ & $1601(1)$ & $18(1)$ \\
\hline$C(5)$ & $8959(1)$ & $973(1)$ & $1867(1)$ & $19(1)$ \\
\hline$C(6)$ & $8118(1)$ & $2075(1)$ & $1891(1)$ & $21(1)$ \\
\hline$C(7)$ & $4092(1)$ & $352(1)$ & $1309(1)$ & $19(1)$ \\
\hline$C(8)$ & $2906(1)$ & $672(1)$ & $1368(1)$ & $22(1)$ \\
\hline $\mathrm{C}(9)$ & $2622(1)$ & $1893(1)$ & $1482(1)$ & $19(1)$ \\
\hline$C(10)$ & $4882(1)$ & $2317(1)$ & $1486(1)$ & $16(1)$ \\
\hline$C(11)$ & $9511(2)$ & $-911(1)$ & $2111(1)$ & $23(1)$ \\
\hline$C(12)$ & $9094(2)$ & $-1857(1)$ & $2356(1)$ & $24(1)$ \\
\hline$C(13)$ & $9101(2)$ & $-1285(2)$ & $2698(1)$ & $32(1)$ \\
\hline$C(14)$ & $9898(2)$ & $-3009(2)$ & $2339(1)$ & $33(1)$ \\
\hline$C(15)$ & $8734(1)$ & $1015(1)$ & $761(1)$ & $17(1)$ \\
\hline$C(16)$ & $8950(1)$ & $-89(1)$ & $543(1)$ & $19(1)$ \\
\hline$C(17)$ & $9675(1)$ & $-1074(1)$ & $641(1)$ & $22(1)$ \\
\hline$C(18)$ & $9801(2)$ & $-2106(2)$ & $441(1)$ & $28(1)$ \\
\hline$C(19)$ & $9211(2)$ & $-2144(2)$ & $142(1)$ & $30(1)$ \\
\hline$C(20)$ & $8503(2)$ & $-1150(2)$ & $40(1)$ & $30(1)$ \\
\hline$C(21)$ & $8376(2)$ & $-118(2)$ & $238(1)$ & $25(1)$ \\
\hline$C(22)$ & $9426(1)$ & $2167(1)$ & $645(1)$ & $18(1)$ \\
\hline$C(23)$ & $10707(2)$ & $2241(2)$ & $692(1)$ & $26(1)$ \\
\hline$C(24)$ & $11357(2)$ & $3282(2)$ & $591(1)$ & $33(1)$ \\
\hline$C(25)$ & $10735(2)$ & $4254(2)$ & $443(1)$ & $33(1)$ \\
\hline$C(26)$ & $9461(2)$ & $4186(2)$ & $398(1)$ & $29(1)$ \\
\hline$C(27)$ & $8808(2)$ & $3146(1)$ & $499(1)$ & $23(1)$ \\
\hline
\end{tabular}




\begin{tabular}{|c|c|c|c|c|}
\hline $\mathrm{O}(1)$ & $6991(1)$ & $442(1)$ & $1573(1)$ & $18(1)$ \\
\hline $\mathrm{O}(2)$ & $7419(1)$ & $1231(1)$ & $771(1)$ & $18(1)$ \\
\hline $\mathrm{O}(3)$ & $9070(1)$ & $741(1)$ & $1087(1)$ & $17(1)$ \\
\hline $\mathrm{O}(4)$ & $8663(1)$ & $4(1)$ & $2084(1)$ & $22(1)$ \\
\hline $\mathrm{O}(5)$ & $10465(1)$ & $-923(1)$ & $1961(1)$ & $40(1)$ \\
\hline $\mathrm{O}(6)$ & $1571(1)$ & $2301(1)$ & $1536(1)$ & $24(1)$ \\
\hline $\mathrm{O}(7)$ & $5731(1)$ & $3015(1)$ & $1553(1)$ & $19(1)$ \\
\hline$N(1)$ & $5073(1)$ & 1154(1) & 1352(1) & $16(1)$ \\
\hline $\mathrm{N}(2)$ & $3655(1)$ & $2637(1)$ & $1531(1)$ & $18(1)$ \\
\hline$C\left(1^{\prime}\right)$ & $-1427(1)$ & $7027(1)$ & $1314(1)$ & $16(1)$ \\
\hline$C\left(2^{\prime}\right)$ & $-2210(1)$ & $6225(1)$ & $1084(1)$ & $16(1)$ \\
\hline$C\left(3^{\prime}\right)$ & $-3284(1)$ & $5739(1)$ & $1298(1)$ & $16(1)$ \\
\hline$C\left(4^{\prime}\right)$ & $-3006(1)$ & $6227(1)$ & $1631(1)$ & $16(1)$ \\
\hline$C\left(5^{\prime}\right)$ & $-4001(1)$ & $6358(1)$ & $1881(1)$ & $18(1)$ \\
\hline$C\left(6^{\prime}\right)$ & $-2986(1)$ & $5422(1)$ & $1929(1)$ & $19(1)$ \\
\hline$C\left(7^{\prime}\right)$ & $815(1)$ & $7301(1)$ & $1426(1)$ & $20(1)$ \\
\hline$C\left(8^{\prime}\right)$ & 1963(1) & $6915(1)$ & $1507(1)$ & $22(1)$ \\
\hline$C\left(9^{\prime}\right)$ & $2177(1)$ & $5613(1)$ & $1555(1)$ & $21(1)$ \\
\hline$C\left(10^{\prime}\right)$ & $-50(1)$ & $5256(1)$ & $1445(1)$ & $17(1)$ \\
\hline$C\left(11^{\prime}\right)$ & $-4684(1)$ & $8343(1)$ & $2028(1)$ & $22(1)$ \\
\hline$C\left(12^{\prime}\right)$ & $-4525(2)$ & $9357(2)$ & $2278(1)$ & $29(1)$ \\
\hline$C\left(13^{\prime}\right)$ & $-4501(2)$ & $8851(2)$ & $2625(1)$ & $35(1)$ \\
\hline$C\left(14^{\prime}\right)$ & $-5524(3)$ & $10328(2)$ & $2235(1)$ & $51(1)$ \\
\hline$C\left(15^{\prime}\right)$ & $-4070(1)$ & $6618(1)$ & $841(1)$ & $16(1)$ \\
\hline$C\left(16^{\prime}\right)$ & $-4864(1)$ & $7684(1)$ & $725(1)$ & $19(1)$ \\
\hline$C\left(17^{\prime}\right)$ & $-6154(2)$ & $7614(2)$ & $756(1)$ & $25(1)$ \\
\hline$C\left(18^{\prime}\right)$ & $-6881(2)$ & $8599(2)$ & $648(1)$ & $34(1)$ \\
\hline$C\left(19^{\prime}\right)$ & $-6332(2)$ & $9637(2)$ & $512(1)$ & $35(1)$ \\
\hline$C\left(20^{\prime}\right)$ & $-5058(2)$ & $9702(2)$ & $482(1)$ & $30(1)$ \\
\hline$C\left(21^{\prime}\right)$ & $-4322(2)$ & $8720(1)$ & $588(1)$ & $23(1)$ \\
\hline$C(22 ')$ & $-4221(1)$ & $5475(1)$ & $625(1)$ & $18(1)$ \\
\hline$C\left(23^{\prime}\right)$ & $-4901(1)$ & $4459(1)$ & $734(1)$ & $21(1)$ \\
\hline
\end{tabular}




$\begin{array}{lrrrr}\mathrm{C}\left(24^{\prime}\right) & -5040(2) & 3426(2) & 534(1) & 26(1) \\ \mathrm{C}\left(25^{\prime}\right) & -4526(2) & 3414(2) & 226(1) & 27(1) \\ \mathrm{C}\left(26^{\prime}\right) & -3865(2) & 4429(2) & 115(1) & 26(1) \\ \mathrm{C}\left(27^{\prime}\right) & -3703(1) & 5456(1) & 314(1) & 21(1) \\ \mathrm{O}\left(1^{\prime}\right) & -2122(1) & 7199(1) & 1602(1) & 19(1) \\ \mathrm{O}\left(2^{\prime}\right) & -2803(1) & 7008(1) & 849(1) & 18(1) \\ \mathrm{O}\left(3^{\prime}\right) & -4368(1) & 6344(1) & 1168(1) & 17(1) \\ \mathrm{O}\left(4^{\prime}\right) & -3926(1) & 7380(1) & 2095(1) & 21(1) \\ \mathrm{O}\left(5^{\prime}\right) & -5375(1) & 8354(1) & 1798(1) & 32(1) \\ \mathrm{O}\left(6^{\prime}\right) & 3180(1) & 5144(1) & 1631(1) & 26(1) \\ \mathrm{O}\left(7^{\prime}\right) & -926(1) & 4543(1) & 1438(1) & 22(1) \\ \mathrm{N}\left(1^{\prime}\right) & -181(1) & 6511(1) & 1383(1) & 17(1) \\ \mathrm{N}\left(2^{\prime}\right) & 1141(1) & 4872(1) & 1512(1) & 20(1)\end{array}$


Table S14. Bond Lengths $[\AA ̊]$ and Angles $\left[^{\circ}\right]$.

\begin{tabular}{|c|c|c|c|}
\hline $\mathrm{C}(1)-\mathrm{O}(1)$ & $1.4189(16)$ & $C(16)-C(21)$ & $1.397(2)$ \\
\hline $\mathrm{C}(1)-\mathrm{N}(1)$ & $1.4740(17)$ & $C(17)-C(18)$ & $1.396(2)$ \\
\hline$C(1)-C(2)$ & $1.5509(19)$ & $C(18)-C(19)$ & $1.382(3)$ \\
\hline $\mathrm{C}(2)-\mathrm{O}(2)$ & $1.4307(16)$ & $C(19)-C(20)$ & $1.384(3)$ \\
\hline$C(2)-C(3)$ & $1.5352(19)$ & $C(20)-C(21)$ & $1.389(2)$ \\
\hline $\mathrm{C}(3)-\mathrm{O}(3)$ & $1.4469(16)$ & $C(22)-C(27)$ & $1.388(2)$ \\
\hline$C(3)-C(4)$ & $1.4875(19)$ & $C(22)-C(23)$ & $1.393(2)$ \\
\hline $\mathrm{C}(4)-\mathrm{O}(1)$ & $1.4186(17)$ & $C(23)-C(24)$ & $1.390(2)$ \\
\hline$C(4)-C(5)$ & $1.4959(19)$ & $C(24)-C(25)$ & $1.387(3)$ \\
\hline$C(4)-C(6)$ & $1.5078(19)$ & $C(25)-C(26)$ & $1.385(3)$ \\
\hline $\mathrm{C}(5)-\mathrm{O}(4)$ & $1.4150(17)$ & $C(26)-C(27)$ & $1.391(2)$ \\
\hline$C(5)-C(6)$ & $1.501(2)$ & $C\left(1^{\prime}\right)-O\left(1^{\prime}\right)$ & $1.4141(17)$ \\
\hline$C(7)-C(8)$ & $1.345(2)$ & $C\left(1^{\prime}\right)-N\left(1^{\prime}\right)$ & $1.4791(17)$ \\
\hline $\mathrm{C}(7)-\mathrm{N}(1)$ & $1.3783(18)$ & $C\left(1^{\prime}\right)-C\left(2^{\prime}\right)$ & $1.5364(19)$ \\
\hline$C(8)-C(9)$ & $1.436(2)$ & $\mathrm{C}\left(2^{\prime}\right)-\mathrm{O}\left(2^{\prime}\right)$ & $1.4329(16)$ \\
\hline $\mathrm{C}(9)-\mathrm{O}(6)$ & $1.2338(18)$ & $C\left(2^{\prime}\right)-C\left(3^{\prime}\right)$ & $1.5439(19)$ \\
\hline $\mathrm{C}(9)-\mathrm{N}(2)$ & $1.3873(18)$ & $\mathrm{C}\left(3^{\prime}\right)-\mathrm{O}\left(3^{\prime}\right)$ & $1.4397(16)$ \\
\hline $\mathrm{C}(10)-\mathrm{O}(7)$ & $1.2173(17)$ & $C\left(3^{\prime}\right)-C\left(4^{\prime}\right)$ & $1.5000(18)$ \\
\hline $\mathrm{C}(10)-\mathrm{N}(2)$ & $1.3771(18)$ & $C\left(4^{\prime}\right)-O\left(1^{\prime}\right)$ & $1.4244(16)$ \\
\hline $\mathrm{C}(10)-\mathrm{N}(1)$ & $1.3898(18)$ & $C\left(4^{\prime}\right)-C\left(5^{\prime}\right)$ & $1.4903(19)$ \\
\hline $\mathrm{C}(11)-\mathrm{O}(5)$ & $1.197(2)$ & $C\left(4^{\prime}\right)-C\left(6^{\prime}\right)$ & $1.5020(18)$ \\
\hline $\mathrm{C}(11)-\mathrm{O}(4)$ & $1.3515(18)$ & $C\left(5^{\prime}\right)-O\left(4^{\prime}\right)$ & $1.4172(17)$ \\
\hline$C(11)-C(12)$ & $1.507(2)$ & $C\left(5^{\prime}\right)-C\left(6^{\prime}\right)$ & $1.503(2)$ \\
\hline$C(12)-C(14)$ & $1.520(2)$ & $C\left(7^{\prime}\right)-C\left(8^{\prime}\right)$ & $1.346(2)$ \\
\hline$C(12)-C(13)$ & $1.538(2)$ & $C\left(7^{\prime}\right)-N\left(1^{\prime}\right)$ & $1.3820(18)$ \\
\hline $\mathrm{C}(15)-\mathrm{O}(3)$ & $1.4183(16)$ & $C\left(8^{\prime}\right)-C\left(9^{\prime}\right)$ & $1.443(2)$ \\
\hline $\mathrm{C}(15)-\mathrm{O}(2)$ & $1.4340(16)$ & $\mathrm{C}\left(9^{\prime}\right)-\mathrm{O}\left(6^{\prime}\right)$ & $1.2315(18)$ \\
\hline$C(15)-C(16)$ & $1.5142(19)$ & $\mathrm{C}\left(9^{\prime}\right)-\mathrm{N}\left(2^{\prime}\right)$ & $1.3845(19)$ \\
\hline$C(15)-C(22)$ & $1.529(2)$ & $\mathrm{C}\left(10^{\prime}\right)-\mathrm{O}\left(7^{\prime}\right)$ & $1.2188(17)$ \\
\hline$C(16)-C(17)$ & $1.381(2)$ & $\mathrm{C}\left(10^{\prime}\right)-\mathrm{N}\left(2^{\prime}\right)$ & $1.3747(18)$ \\
\hline
\end{tabular}




\begin{tabular}{|c|c|c|c|}
\hline $\mathrm{C}\left(10^{\prime}\right)-\mathrm{N}\left(1^{\prime}\right)$ & $1.3903(18)$ & $C\left(16^{\prime}\right)-C\left(17^{\prime}\right)$ & $1.395(2)$ \\
\hline$C\left(11^{\prime}\right)-O\left(5^{\prime}\right)$ & $1.200(2)$ & $C\left(17^{\prime}\right)-C\left(18^{\prime}\right)$ & $1.396(2)$ \\
\hline$C\left(11^{\prime}\right)-O\left(4^{\prime}\right)$ & $1.3531(18)$ & $C\left(18^{\prime}\right)-C\left(19^{\prime}\right)$ & $1.386(3)$ \\
\hline$C\left(11^{\prime}\right)-C\left(12^{\prime}\right)$ & $1.516(2)$ & $C\left(19^{\prime}\right)-C\left(20^{\prime}\right)$ & $1.378(3)$ \\
\hline $\mathrm{C}\left(12^{\prime}\right)-\mathrm{C}\left(14^{\prime}\right)$ & $1.514(3)$ & $C\left(20^{\prime}\right)-C\left(21^{\prime}\right)$ & $1.396(2)$ \\
\hline$C\left(12^{\prime}\right)-C\left(13^{\prime}\right)$ & $1.529(2)$ & $C\left(22^{\prime}\right)-C\left(23^{\prime}\right)$ & $1.394(2)$ \\
\hline$C\left(15^{\prime}\right)-O\left(3^{\prime}\right)$ & $1.4118(16)$ & $C\left(22^{\prime}\right)-C\left(27^{\prime}\right)$ & $1.395(2)$ \\
\hline$C\left(15^{\prime}\right)-O\left(2^{\prime}\right)$ & $1.4260(16)$ & $C\left(23^{\prime}\right)-C\left(24^{\prime}\right)$ & $1.396(2)$ \\
\hline$C\left(15^{\prime}\right)-C\left(16^{\prime}\right)$ & $1.514(2)$ & $C\left(24^{\prime}\right)-C\left(25^{\prime}\right)$ & $1.385(2)$ \\
\hline$C\left(15^{\prime}\right)-C\left(22^{\prime}\right)$ & $1.5323(19)$ & $C\left(25^{\prime}\right)-C\left(26^{\prime}\right)$ & $1.386(3)$ \\
\hline$C\left(16^{\prime}\right)-C\left(21^{\prime}\right)$ & $1.386(2)$ & $C\left(26^{\prime}\right)-C\left(27^{\prime}\right)$ & $1.392(2)$ \\
\hline $\mathrm{O}(1)-\mathrm{C}(1)-\mathrm{N}(1)$ & $110.86(11)$ & $C(7)-C(8)-C(9)$ & $119.90(14)$ \\
\hline $\mathrm{O}(1)-\mathrm{C}(1)-\mathrm{C}(2)$ & $108.06(11)$ & $\mathrm{O}(6)-\mathrm{C}(9)-\mathrm{N}(2)$ & $119.95(13)$ \\
\hline$N(1)-C(1)-C(2)$ & $114.31(11)$ & $\mathrm{O}(6)-\mathrm{C}(9)-\mathrm{C}(8)$ & $125.66(14)$ \\
\hline $\mathrm{O}(2)-\mathrm{C}(2)-\mathrm{C}(3)$ & $105.08(10)$ & $N(2)-C(9)-C(8)$ & $114.39(13)$ \\
\hline $\mathrm{O}(2)-\mathrm{C}(2)-\mathrm{C}(1)$ & $110.87(11)$ & $\mathrm{O}(7)-\mathrm{C}(10)-\mathrm{N}(2)$ & $122.13(13)$ \\
\hline$C(3)-C(2)-C(1)$ & $103.10(11)$ & $\mathrm{O}(7)-\mathrm{C}(10)-\mathrm{N}(1)$ & $122.87(12)$ \\
\hline $\mathrm{O}(3)-\mathrm{C}(3)-\mathrm{C}(4)$ & $112.77(11)$ & $N(2)-C(10)-N(1)$ & $114.99(12)$ \\
\hline $\mathrm{O}(3)-\mathrm{C}(3)-\mathrm{C}(2)$ & $101.30(10)$ & $\mathrm{O}(5)-\mathrm{C}(11)-\mathrm{O}(4)$ & $122.93(15)$ \\
\hline$C(4)-C(3)-C(2)$ & $103.00(11)$ & $\mathrm{O}(5)-\mathrm{C}(11)-\mathrm{C}(12)$ & $126.38(15)$ \\
\hline $\mathrm{O}(1)-\mathrm{C}(4)-\mathrm{C}(3)$ & $108.56(11)$ & $\mathrm{O}(4)-\mathrm{C}(11)-\mathrm{C}(12)$ & $110.67(13)$ \\
\hline $\mathrm{O}(1)-\mathrm{C}(4)-\mathrm{C}(5)$ & $118.33(12)$ & $C(11)-C(12)-C(14)$ & $110.98(14)$ \\
\hline$C(3)-C(4)-C(5)$ & $123.88(12)$ & $C(11)-C(12)-C(13)$ & $109.64(13)$ \\
\hline $\mathrm{O}(1)-\mathrm{C}(4)-\mathrm{C}(6)$ & $118.18(12)$ & $C(14)-C(12)-C(13)$ & $111.78(13)$ \\
\hline$C(3)-C(4)-C(6)$ & $121.01(12)$ & $\mathrm{O}(3)-\mathrm{C}(15)-\mathrm{O}(2)$ & $105.08(10)$ \\
\hline$C(5)-C(4)-C(6)$ & $59.94(10)$ & $\mathrm{O}(3)-\mathrm{C}(15)-\mathrm{C}(16)$ & $110.81(11)$ \\
\hline $\mathrm{O}(4)-\mathrm{C}(5)-\mathrm{C}(4)$ & $116.62(12)$ & $\mathrm{O}(2)-\mathrm{C}(15)-\mathrm{C}(16)$ & $107.24(11)$ \\
\hline $\mathrm{O}(4)-\mathrm{C}(5)-\mathrm{C}(6)$ & $114.39(12)$ & $\mathrm{O}(3)-\mathrm{C}(15)-\mathrm{C}(22)$ & $109.97(11)$ \\
\hline$C(4)-C(5)-C(6)$ & $60.42(9)$ & $\mathrm{O}(2)-\mathrm{C}(15)-\mathrm{C}(22)$ & $110.80(11)$ \\
\hline$C(5)-C(6)-C(4)$ & $59.64(9)$ & $C(16)-C(15)-C(22)$ & $112.65(11)$ \\
\hline $\mathrm{C}(8)-\mathrm{C}(7)-\mathrm{N}(1)$ & $122.63(13)$ & $C(17)-C(16)-C(21)$ & $119.65(14)$ \\
\hline
\end{tabular}




\begin{tabular}{|c|c|c|c|}
\hline$C(17)-C(16)-C(15)$ & $121.57(13)$ & $\mathrm{O}\left(1^{\prime}\right)-\mathrm{C}\left(4^{\prime}\right)-\mathrm{C}\left(5^{\prime}\right)$ & $117.74(11)$ \\
\hline$C(21)-C(16)-C(15)$ & $118.77(14)$ & $\mathrm{O}\left(1^{\prime}\right)-\mathrm{C}\left(4^{\prime}\right)-\mathrm{C}\left(3^{\prime}\right)$ & $108.54(11)$ \\
\hline$C(16)-C(17)-C(18)$ & $120.06(15)$ & $C\left(5^{\prime}\right)-C\left(4^{\prime}\right)-C\left(3^{\prime}\right)$ & $121.40(12)$ \\
\hline $\mathrm{C}(19)-\mathrm{C}(18)-\mathrm{C}(17)$ & $120.22(16)$ & $\mathrm{O}\left(1^{\prime}\right)-\mathrm{C}\left(4^{\prime}\right)-\mathrm{C}\left(6^{\prime}\right)$ & $119.17(11)$ \\
\hline$C(18)-C(19)-C(20)$ & $119.85(15)$ & $C\left(5^{\prime}\right)-C\left(4^{\prime}\right)-C\left(6^{\prime}\right)$ & $60.32(9)$ \\
\hline$C(19)-C(20)-C(21)$ & $120.27(15)$ & $C\left(3^{\prime}\right)-C\left(4^{\prime}\right)-C\left(6^{\prime}\right)$ & $122.88(12)$ \\
\hline$C(20)-C(21)-C(16)$ & $119.92(16)$ & $\mathrm{O}\left(4^{\prime}\right)-\mathrm{C}\left(5^{\prime}\right)-\mathrm{C}\left(4^{\prime}\right)$ & $117.54(12)$ \\
\hline$C(27)-C(22)-C(23)$ & $119.22(14)$ & $\mathrm{O}\left(4^{\prime}\right)-\mathrm{C}\left(5^{\prime}\right)-\mathrm{C}\left(6^{\prime}\right)$ & $113.88(12)$ \\
\hline$C(27)-C(22)-C(15)$ & $121.74(13)$ & $C\left(4^{\prime}\right)-C\left(5^{\prime}\right)-C\left(6^{\prime}\right)$ & $60.22(9)$ \\
\hline$C(23)-C(22)-C(15)$ & $119.03(14)$ & $C\left(4^{\prime}\right)-C\left(6^{\prime}\right)-C\left(5^{\prime}\right)$ & $59.45(9)$ \\
\hline$C(24)-C(23)-C(22)$ & $120.21(16)$ & $\mathrm{C}\left(8^{\prime}\right)-\mathrm{C}\left(7^{\prime}\right)-\mathrm{N}\left(1^{\prime}\right)$ & $123.35(13)$ \\
\hline$C(25)-C(24)-C(23)$ & $120.25(16)$ & $C\left(7^{\prime}\right)-C\left(8^{\prime}\right)-C\left(9^{\prime}\right)$ & $118.96(14)$ \\
\hline$C(26)-C(25)-C(24)$ & $119.72(16)$ & $\mathrm{O}\left(6^{\prime}\right)-\mathrm{C}\left(9^{\prime}\right)-\mathrm{N}\left(2^{\prime}\right)$ & $119.88(14)$ \\
\hline$C(25)-C(26)-C(27)$ & $120.13(16)$ & $\mathrm{O}\left(6^{\prime}\right)-\mathrm{C}\left(9^{\prime}\right)-\mathrm{C}\left(8^{\prime}\right)$ & $125.24(14)$ \\
\hline$C(22)-C(27)-C(26)$ & $120.46(15)$ & $\mathrm{N}\left(2^{\prime}\right)-\mathrm{C}\left(9^{\prime}\right)-\mathrm{C}\left(8^{\prime}\right)$ & $114.88(13)$ \\
\hline$C(4)-O(1)-C(1)$ & $108.76(10)$ & $\mathrm{O}\left(7^{\prime}\right)-\mathrm{C}\left(10^{\prime}\right)-\mathrm{N}\left(2^{\prime}\right)$ & $122.21(13)$ \\
\hline $\mathrm{C}(2)-\mathrm{O}(2)-\mathrm{C}(15)$ & $106.44(10)$ & $\mathrm{O}\left(7^{\prime}\right)-\mathrm{C}\left(10^{\prime}\right)-\mathrm{N}\left(1^{\prime}\right)$ & $122.53(13)$ \\
\hline $\mathrm{C}(15)-\mathrm{O}(3)-\mathrm{C}(3)$ & $102.78(10)$ & $N\left(2^{\prime}\right)-C\left(10^{\prime}\right)-N\left(1^{\prime}\right)$ & $115.26(12)$ \\
\hline $\mathrm{C}(11)-\mathrm{O}(4)-\mathrm{C}(5)$ & $116.38(12)$ & $\mathrm{O}\left(5^{\prime}\right)-\mathrm{C}\left(11^{\prime}\right)-\mathrm{O}\left(4^{\prime}\right)$ & $122.88(14)$ \\
\hline$C(7)-N(1)-C(10)$ & $120.73(12)$ & $\mathrm{O}\left(5^{\prime}\right)-\mathrm{C}\left(11^{\prime}\right)-\mathrm{C}\left(12^{\prime}\right)$ & $126.55(15)$ \\
\hline$C(7)-N(1)-C(1)$ & $119.55(11)$ & $\mathrm{O}\left(4^{\prime}\right)-\mathrm{C}\left(11^{\prime}\right)-\mathrm{C}\left(12^{\prime}\right)$ & $110.57(13)$ \\
\hline$C(10)-N(1)-C(1)$ & $119.53(11)$ & $C\left(14^{\prime}\right)-C\left(12^{\prime}\right)-C\left(11^{\prime}\right)$ & ) $110.23(16)$ \\
\hline$C(10)-N(2)-C(9)$ & $126.97(12)$ & $C\left(14^{\prime}\right)-C\left(12^{\prime}\right)-C\left(13^{\prime}\right)$ & ) $111.69(14)$ \\
\hline $\mathrm{O}\left(1^{\prime}\right)-\mathrm{C}\left(1^{\prime}\right)-\mathrm{N}\left(1^{\prime}\right)$ & $111.59(11)$ & $\mathrm{C}\left(11^{\prime}\right)-\mathrm{C}\left(12^{\prime}\right)-\mathrm{C}\left(13^{\prime}\right)$ & ) $112.07(14)$ \\
\hline $\mathrm{O}\left(1^{\prime}\right)-\mathrm{C}\left(1^{\prime}\right)-\mathrm{C}\left(2^{\prime}\right)$ & $107.58(11)$ & $\mathrm{O}\left(3^{\prime}\right)-\mathrm{C}\left(15^{\prime}\right)-\mathrm{O}\left(2^{\prime}\right)$ & $104.86(10)$ \\
\hline $\mathrm{N}\left(1^{\prime}\right)-\mathrm{C}\left(1^{\prime}\right)-\mathrm{C}\left(2^{\prime}\right)$ & $113.63(11)$ & $\mathrm{O}\left(3^{\prime}\right)-\mathrm{C}\left(15^{\prime}\right)-\mathrm{C}\left(16^{\prime}\right)$ & $109.34(11)$ \\
\hline$O\left(2^{\prime}\right)-C\left(2^{\prime}\right)-C\left(1^{\prime}\right)$ & $108.90(11)$ & $\mathrm{O}\left(2^{\prime}\right)-\mathrm{C}\left(15^{\prime}\right)-\mathrm{C}\left(16^{\prime}\right)$ & $108.69(11)$ \\
\hline $\mathrm{O}\left(2^{\prime}\right)-\mathrm{C}\left(2^{\prime}\right)-\mathrm{C}\left(3^{\prime}\right)$ & $104.57(11)$ & $\mathrm{O}\left(3^{\prime}\right)-\mathrm{C}\left(15^{\prime}\right)-\mathrm{C}\left(22^{\prime}\right)$ & $110.90(11)$ \\
\hline$C\left(1^{\prime}\right)-C\left(2^{\prime}\right)-C\left(3^{\prime}\right)$ & $104.59(11)$ & $\mathrm{O}\left(2^{\prime}\right)-\mathrm{C}\left(15^{\prime}\right)-\mathrm{C}\left(22^{\prime}\right)$ & $110.75(11)$ \\
\hline $\mathrm{O}\left(3^{\prime}\right)-\mathrm{C}\left(3^{\prime}\right)-\mathrm{C}\left(4^{\prime}\right)$ & $109.86(11)$ & $C\left(16^{\prime}\right)-C\left(15^{\prime}\right)-C\left(22^{\prime}\right)$ & ) $112.03(11)$ \\
\hline $\mathrm{O}\left(3^{\prime}\right)-\mathrm{C}\left(3^{\prime}\right)-\mathrm{C}\left(2^{\prime}\right)$ & $103.83(10)$ & $C\left(21^{\prime}\right)-C\left(16^{\prime}\right)-C\left(17^{\prime}\right)$ & ) $119.97(15)$ \\
\hline$C\left(4^{\prime}\right)-C\left(3^{\prime}\right)-C\left(2^{\prime}\right)$ & $104.56(11)$ & $C\left(21^{\prime}\right)-C\left(16^{\prime}\right)-C\left(15^{\prime}\right)$ & ) $120.61(13)$ \\
\hline
\end{tabular}


$C\left(17^{\prime}\right)-C\left(16^{\prime}\right)-C\left(15^{\prime}\right)$ 119.41(14)

$C\left(16^{\prime}\right)-C\left(17^{\prime}\right)-C\left(18^{\prime}\right)$ 119.07(16)

$C\left(19^{\prime}\right)-C\left(18^{\prime}\right)-C\left(17^{\prime}\right)$ 120.69(16)

$\mathrm{C}\left(20^{\prime}\right)-\mathrm{C}\left(19^{\prime}\right)-\mathrm{C}\left(18^{\prime}\right)$ 120.07(16)

$\mathrm{C}\left(19^{\prime}\right)-\mathrm{C}\left(20^{\prime}\right)-\mathrm{C}\left(21^{\prime}\right)$ 119.77(17)

$\mathrm{C}\left(16^{\prime}\right)-\mathrm{C}\left(21^{\prime}\right)-\mathrm{C}\left(20^{\prime}\right)$ 120.42(15)

$\mathrm{C}\left(23^{\prime}\right)-\mathrm{C}\left(22^{\prime}\right)-\mathrm{C}\left(27^{\prime}\right)$ 119.39(14)

$C\left(23^{\prime}\right)-C\left(22^{\prime}\right)-C\left(15^{\prime}\right)$ 120.58(13)

C(27')-C(22')-C(15') 120.00(13)

C(22')-C(23')-C(24') 120.14(14)

$C\left(25^{\prime}\right)-C\left(24^{\prime}\right)-C\left(23^{\prime}\right)$ 120.19(15)
$\mathrm{C}\left(24^{\prime}\right)-\mathrm{C}\left(25^{\prime}\right)-\mathrm{C}\left(26^{\prime}\right)$ 119.81(15)

$\mathrm{C}\left(25^{\prime}\right)-\mathrm{C}\left(26^{\prime}\right)-\mathrm{C}\left(27^{\prime}\right)$ 120.41(15)

C(26')-C(27')-C(22') 120.04(14)

$\mathrm{C}\left(1^{\prime}\right)-\mathrm{O}\left(1^{\prime}\right)-\mathrm{C}\left(4^{\prime}\right) \quad$ 108.92(10)

$\mathrm{C}\left(15^{\prime}\right)-\mathrm{O}\left(2^{\prime}\right)-\mathrm{C}\left(2^{\prime}\right) \quad$ 105.42(10)

$\mathrm{C}\left(15^{\prime}\right)-\mathrm{O}\left(3^{\prime}\right)-\mathrm{C}\left(3^{\prime}\right) \quad$ 105.31(10)

$\mathrm{C}\left(11^{\prime}\right)-\mathrm{O}\left(4^{\prime}\right)-\mathrm{C}\left(5^{\prime}\right) \quad 116.08(11)$

$\mathrm{C}\left(7^{\prime}\right)-\mathrm{N}\left(1^{\prime}\right)-\mathrm{C}\left(10^{\prime}\right) \quad 120.28(12)$

$\mathrm{C}\left(7^{\prime}\right)-\mathrm{N}\left(1^{\prime}\right)-\mathrm{C}\left(1^{\prime}\right) \quad 119.46(11)$

$\mathrm{C}\left(10^{\prime}\right)-\mathrm{N}\left(1^{\prime}\right)-\mathrm{C}\left(1^{\prime}\right) \quad 119.76(11)$

$\mathrm{C}\left(10^{\prime}\right)-\mathrm{N}\left(2^{\prime}\right)-\mathrm{C}\left(9^{\prime}\right) \quad 126.90(13)$

Table S15. Torsion Angles [ $\left.{ }^{\circ}\right]$.

\begin{tabular}{lclc}
\hline $\mathrm{O}(1)-\mathrm{C}(1)-\mathrm{C}(2)-\mathrm{O}(2)$ & $123.30(11)$ & $\mathrm{C}(3)-\mathrm{C}(4)-\mathrm{C}(5)-\mathrm{C}(6)$ & $109.17(15)$ \\
$\mathrm{N}(1)-\mathrm{C}(1)-\mathrm{C}(2)-\mathrm{O}(2)$ & $-112.76(12)$ & $\mathrm{O}(4)-\mathrm{C}(5)-\mathrm{C}(6)-\mathrm{C}(4)$ & $-107.96(13)$ \\
$\mathrm{O}(1)-\mathrm{C}(1)-\mathrm{C}(2)-\mathrm{C}(3)$ & $11.31(13)$ & $\mathrm{O}(1)-\mathrm{C}(4)-\mathrm{C}(6)-\mathrm{C}(5)$ & $108.18(14)$ \\
$\mathrm{N}(1)-\mathrm{C}(1)-\mathrm{C}(2)-\mathrm{C}(3)$ & $135.25(11)$ & $\mathrm{C}(3)-\mathrm{C}(4)-\mathrm{C}(6)-\mathrm{C}(5)$ & $-113.81(15)$ \\
$\mathrm{O}(2)-\mathrm{C}(2)-\mathrm{C}(3)-\mathrm{O}(3)$ & $-23.49(13)$ & $\mathrm{N}(1)-\mathrm{C}(7)-\mathrm{C}(8)-\mathrm{C}(9)$ & $0.2(2)$ \\
$\mathrm{C}(1)-\mathrm{C}(2)-\mathrm{C}(3)-\mathrm{O}(3)$ & $92.70(11)$ & $\mathrm{C}(7)-\mathrm{C}(8)-\mathrm{C}(9)-\mathrm{O}(6)$ & $177.65(15)$ \\
$\mathrm{O}(2)-\mathrm{C}(2)-\mathrm{C}(3)-\mathrm{C}(4)$ & $-140.32(11)$ & $\mathrm{C}(7)-\mathrm{C}(8)-\mathrm{C}(9)-\mathrm{N}(2)$ & $-2.1(2)$ \\
$\mathrm{C}(1)-\mathrm{C}(2)-\mathrm{C}(3)-\mathrm{C}(4)$ & $-24.12(13)$ & $\mathrm{O}(5)-\mathrm{C}(11)-\mathrm{C}(12)-\mathrm{C}(14)$ & $-12.9(2)$ \\
$\mathrm{O}(3)-\mathrm{C}(3)-\mathrm{C}(4)-\mathrm{O}(1)$ & $-78.31(14)$ & $\mathrm{O}(4)-\mathrm{C}(11)-\mathrm{C}(12)-\mathrm{C}(14)$ & $168.45(13)$ \\
$\mathrm{C}(2)-\mathrm{C}(3)-\mathrm{C}(4)-\mathrm{O}(1)$ & $30.06(14)$ & $\mathrm{O}(5)-\mathrm{C}(11)-\mathrm{C}(12)-\mathrm{C}(13)$ & $111.07(19)$ \\
$\mathrm{O}(3)-\mathrm{C}(3)-\mathrm{C}(4)-\mathrm{C}(5)$ & $67.63(17)$ & $\mathrm{O}(4)-\mathrm{C}(11)-\mathrm{C}(12)-\mathrm{C}(13)$ & $-67.58(17)$ \\
$\mathrm{C}(2)-\mathrm{C}(3)-\mathrm{C}(4)-\mathrm{C}(5)$ & $176.00(13)$ & $\mathrm{O}(3)-\mathrm{C}(15)-\mathrm{C}(16)-\mathrm{C}(17)$ & $-15.48(18)$ \\
$\mathrm{O}(3)-\mathrm{C}(3)-\mathrm{C}(4)-\mathrm{C}(6)$ & $140.16(13)$ & $\mathrm{O}(2)-\mathrm{C}(15)-\mathrm{C}(16)-\mathrm{C}(17)$ & $-129.64(14)$ \\
$\mathrm{C}(2)-\mathrm{C}(3)-\mathrm{C}(4)-\mathrm{C}(6)$ & $-111.48(14)$ & $\mathrm{C}(22)-\mathrm{C}(15)-\mathrm{C}(16)-\mathrm{C}(17)$ & $108.19(15)$ \\
$\mathrm{O}(1)-\mathrm{C}(4)-\mathrm{C}(5)-\mathrm{O}(4)$ & $-3.65(19)$ & $\mathrm{O}(3)-\mathrm{C}(15)-\mathrm{C}(16)-\mathrm{C}(21)$ & $163.02(13)$ \\
$\mathrm{C}(3)-\mathrm{C}(4)-\mathrm{C}(5)-\mathrm{O}(4)$ & $-146.55(13)$ & $\mathrm{O}(2)-\mathrm{C}(15)-\mathrm{C}(16)-\mathrm{C}(21)$ & $48.86(17)$ \\
$\mathrm{C}(6)-\mathrm{C}(4)-\mathrm{C}(5)-\mathrm{O}(4)$ & $104.28(14)$ & $\mathrm{C}(22)-\mathrm{C}(15)-\mathrm{C}(16)-\mathrm{C}(21)$ & $-73.31(17)$ \\
$\mathrm{O}(1)-\mathrm{C}(4)-\mathrm{C}(5)-\mathrm{C}(6)$ & $-107.93(14)$ & $\mathrm{C}(21)-\mathrm{C}(16)-\mathrm{C}(17)-\mathrm{C}(18)$ & $-1.9(2)$ \\
& & &
\end{tabular}




\begin{tabular}{|c|c|c|c|}
\hline$C(15)-C(16)-C(17)-C(18)$ & $176.57(13)$ & $C(16)-C(15)-O(3)-C(3)$ & $-159.32(11)$ \\
\hline$C(16)-C(17)-C(18)-C(19)$ & $0.6(2)$ & $C(22)-C(15)-O(3)-C(3)$ & $75.49(12)$ \\
\hline$C(17)-C(18)-C(19)-C(20)$ & $0.6(2)$ & $C(4)-C(3)-O(3)-C(15)$ & $149.97(12)$ \\
\hline$C(18)-C(19)-C(20)-C(21)$ & $-0.5(2)$ & $C(2)-C(3)-O(3)-C(15)$ & $40.54(12)$ \\
\hline$C(19)-C(20)-C(21)-C(16)$ & $-0.9(2)$ & $\mathrm{O}(5)-\mathrm{C}(11)-\mathrm{O}(4)-\mathrm{C}(5)$ & $-0.3(2)$ \\
\hline$C(17)-C(16)-C(21)-C(20)$ & $2.1(2)$ & $C(12)-C(11)-O(4)-C(5)$ & $178.39(12)$ \\
\hline$C(15)-C(16)-C(21)-C(20)$ & $-176.46(14)$ & $C(4)-C(5)-O(4)-C(11)$ & $123.28(14)$ \\
\hline $\mathrm{O}(3)-\mathrm{C}(15)-\mathrm{C}(22)-\mathrm{C}(27)$ & $-129.63(14)$ & $C(6)-C(5)-O(4)-C(11)$ & $-168.99(12)$ \\
\hline $\mathrm{O}(2)-\mathrm{C}(15)-\mathrm{C}(22)-\mathrm{C}(27)$ & $-13.90(18)$ & $C(8)-C(7)-N(1)-C(10)$ & $4.9(2)$ \\
\hline$C(16)-C(15)-C(22)-C(27)$ & $106.24(15)$ & $C(8)-C(7)-N(1)-C(1)$ & $179.89(14)$ \\
\hline $\mathrm{O}(3)-\mathrm{C}(15)-\mathrm{C}(22)-\mathrm{C}(23)$ & $49.30(17)$ & $\mathrm{O}(7)-\mathrm{C}(10)-\mathrm{N}(1)-\mathrm{C}(7)$ & $173.90(13)$ \\
\hline $\mathrm{O}(2)-\mathrm{C}(15)-\mathrm{C}(22)-\mathrm{C}(23)$ & $165.03(13)$ & $N(2)-C(10)-N(1)-C(7)$ & $-7.36(19)$ \\
\hline$C(16)-C(15)-C(22)-C(23)$ & $-74.84(17)$ & $\mathrm{O}(7)-\mathrm{C}(10)-\mathrm{N}(1)-\mathrm{C}(1)$ & $-1.1(2)$ \\
\hline$C(27)-C(22)-C(23)-C(24)$ & $-0.5(2)$ & $N(2)-C(10)-N(1)-C(1)$ & $177.61(12)$ \\
\hline$C(15)-C(22)-C(23)-C(24)$ & $-179.46(14)$ & $O(1)-C(1)-N(1)-C(7)$ & $-101.74(14)$ \\
\hline$C(22)-C(23)-C(24)-C(25)$ & $0.1(3)$ & $C(2)-C(1)-N(1)-C(7)$ & $135.83(13)$ \\
\hline$C(23)-C(24)-C(25)-C(26)$ & $0.3(3)$ & $O(1)-C(1)-N(1)-C(10)$ & $73.35(15)$ \\
\hline$C(24)-C(25)-C(26)-C(27)$ & $-0.2(3)$ & $C(2)-C(1)-N(1)-C(10)$ & $-49.07(16)$ \\
\hline$C(23)-C(22)-C(27)-C(26)$ & $0.6(2)$ & $\mathrm{O}(7)-\mathrm{C}(10)-\mathrm{N}(2)-\mathrm{C}(9)$ & $-175.62(14)$ \\
\hline$C(15)-C(22)-C(27)-C(26)$ & $179.53(14)$ & $N(1)-C(10)-N(2)-C(9)$ & $5.6(2)$ \\
\hline$C(25)-C(26)-C(27)-C(22)$ & $-0.3(3)$ & $\mathrm{O}(6)-\mathrm{C}(9)-\mathrm{N}(2)-\mathrm{C}(10)$ & $179.24(14)$ \\
\hline$C(3)-C(4)-O(1)-C(1)$ & $-23.78(14)$ & $C(8)-C(9)-N(2)-C(10)$ & $-1.0(2)$ \\
\hline $\mathrm{C}(5)-\mathrm{C}(4)-\mathrm{O}(1)-\mathrm{C}(1)$ & $-171.89(12)$ & $\mathrm{O}\left(1^{\prime}\right)-\mathrm{C}\left(1^{\prime}\right)-\mathrm{C}\left(2^{\prime}\right)-\mathrm{O}\left(2^{\prime}\right)$ & $99.72(12)$ \\
\hline $\mathrm{C}(6)-\mathrm{C}(4)-\mathrm{O}(1)-\mathrm{C}(1)$ & $119.01(13)$ & $\mathrm{N}\left(1^{\prime}\right)-\mathrm{C}\left(1^{\prime}\right)-\mathrm{C}\left(2^{\prime}\right)-\mathrm{O}\left(2^{\prime}\right)$ & $-136.23(11)$ \\
\hline$N(1)-C(1)-O(1)-C(4)$ & $-118.93(12)$ & $\mathrm{O}\left(1^{\prime}\right)-\mathrm{C}\left(1^{\prime}\right)-\mathrm{C}\left(2^{\prime}\right)-\mathrm{C}\left(3^{\prime}\right)$ & $-11.62(14)$ \\
\hline$C(2)-C(1)-O(1)-C(4)$ & $7.05(14)$ & $\mathrm{N}\left(1^{\prime}\right)-\mathrm{C}\left(1^{\prime}\right)-\mathrm{C}\left(2^{\prime}\right)-\mathrm{C}\left(3^{\prime}\right)$ & $112.43(12)$ \\
\hline $\mathrm{C}(3)-\mathrm{C}(2)-\mathrm{O}(2)-\mathrm{C}(15)$ & $-2.26(14)$ & $\mathrm{O}\left(2^{\prime}\right)-\mathrm{C}\left(2^{\prime}\right)-\mathrm{C}\left(3^{\prime}\right)-\mathrm{O}\left(3^{\prime}\right)$ & $-2.27(13)$ \\
\hline$C(1)-C(2)-O(2)-C(15)$ & $-112.98(12)$ & $\mathrm{C}\left(1^{\prime}\right)-\mathrm{C}\left(2^{\prime}\right)-\mathrm{C}\left(3^{\prime}\right)-\mathrm{O}\left(3^{\prime}\right)$ & 112.16(11) \\
\hline $\mathrm{O}(3)-\mathrm{C}(15)-\mathrm{O}(2)-\mathrm{C}(2)$ & $28.22(13)$ & $\mathrm{O}\left(2^{\prime}\right)-\mathrm{C}\left(2^{\prime}\right)-\mathrm{C}\left(3^{\prime}\right)-\mathrm{C}\left(4^{\prime}\right)$ & $-117.45(12)$ \\
\hline $\mathrm{C}(16)-\mathrm{C}(15)-\mathrm{O}(2)-\mathrm{C}(2)$ & $146.18(11)$ & $C\left(1^{\prime}\right)-C\left(2^{\prime}\right)-C\left(3^{\prime}\right)-C\left(4^{\prime}\right)$ & $-3.02(14)$ \\
\hline $\mathrm{C}(22)-\mathrm{C}(15)-\mathrm{O}(2)-\mathrm{C}(2)$ & $-90.51(12)$ & $\mathrm{O}\left(3^{\prime}\right)-\mathrm{C}\left(3^{\prime}\right)-\mathrm{C}\left(4^{\prime}\right)-\mathrm{O}\left(1^{\prime}\right)$ & $-94.15(13)$ \\
\hline $\mathrm{O}(2)-\mathrm{C}(15)-\mathrm{O}(3)-\mathrm{C}(3)$ & $-43.81(13)$ & $\mathrm{C}\left(2^{\prime}\right)-\mathrm{C}\left(3^{\prime}\right)-\mathrm{C}\left(4^{\prime}\right)-\mathrm{O}\left(1^{\prime}\right)$ & $16.73(14)$ \\
\hline \multicolumn{4}{|c|}{ 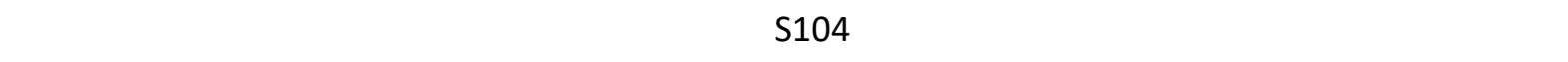 } \\
\hline
\end{tabular}




\begin{tabular}{|c|c|c|c|}
\hline $\mathrm{O}\left(3^{\prime}\right)-\mathrm{C}\left(3^{\prime}\right)-\mathrm{C}\left(4^{\prime}\right)-\mathrm{C}\left(5^{\prime}\right)$ & $47.10(17)$ & \multicolumn{2}{|c|}{$C\left(19^{\prime}\right)-C\left(20^{\prime}\right)-C\left(21^{\prime}\right)-C\left(16^{\prime}\right) \quad-0.4(2)$} \\
\hline$C\left(2^{\prime}\right)-C\left(3^{\prime}\right)-C\left(4^{\prime}\right)-C\left(5^{\prime}\right)$ & $157.99(13)$ & \multicolumn{2}{|c|}{$\mathrm{O}\left(3^{\prime}\right)-\mathrm{C}\left(15^{\prime}\right)-\mathrm{C}\left(22^{\prime}\right)-\mathrm{C}\left(23^{\prime}\right) \quad 16.79(18)$} \\
\hline $\mathrm{O}\left(3^{\prime}\right)-\mathrm{C}\left(3^{\prime}\right)-\mathrm{C}\left(4^{\prime}\right)-\mathrm{C}\left(6^{\prime}\right)$ & $119.88(14)$ & \multicolumn{2}{|c|}{$\mathrm{O}\left(2^{\prime}\right)-\mathrm{C}\left(15^{\prime}\right)-\mathrm{C}\left(22^{\prime}\right)-\mathrm{C}\left(23^{\prime}\right) \quad 132.79(13)$} \\
\hline$C\left(2^{\prime}\right)-C\left(3^{\prime}\right)-C\left(4^{\prime}\right)-C\left(6^{\prime}\right)$ & $-129.24(13)$ & \multicolumn{2}{|c|}{$C\left(16^{\prime}\right)-C\left(15^{\prime}\right)-C\left(22^{\prime}\right)-C\left(23^{\prime}\right)-105.68(15)$} \\
\hline $\mathrm{O}\left(1^{\prime}\right)-\mathrm{C}\left(4^{\prime}\right)-\mathrm{C}\left(5^{\prime}\right)-\mathrm{O}\left(4^{\prime}\right)$ & $-6.35(18)$ & \multicolumn{2}{|c|}{$\mathrm{O}\left(3^{\prime}\right)-\mathrm{C}\left(15^{\prime}\right)-\mathrm{C}\left(222^{\prime}\right)-\mathrm{C}\left(27^{\prime}\right)-164.90(12)$} \\
\hline$C\left(3^{\prime}\right)-C\left(4^{\prime}\right)-C\left(5^{\prime}\right)-O\left(4^{\prime}\right)$ & $-144.24(13)$ & $\mathrm{O}\left(2^{\prime}\right)-\mathrm{C}\left(15^{\prime}\right)-\mathrm{C}\left(22^{\prime}\right)-\mathrm{C}\left(27^{\prime}\right)$ & $-48.91(17)$ \\
\hline$C\left(6^{\prime}\right)-C\left(4^{\prime}\right)-C\left(5^{\prime}\right)-O\left(4^{\prime}\right)$ & $103.17(14)$ & \multicolumn{2}{|c|}{$C\left(16^{\prime}\right)-C\left(15^{\prime}\right)-C\left(22^{\prime}\right)-C\left(27^{\prime}\right) 72.62(16)$} \\
\hline $\mathrm{O}\left(1^{\prime}\right)-\mathrm{C}\left(4^{\prime}\right)-\mathrm{C}\left(5^{\prime}\right)-\mathrm{C}\left(6^{\prime}\right)$ & $-109.51(14)$ & $C\left(27^{\prime}\right)-C\left(22^{\prime}\right)-C\left(23^{\prime}\right)-C\left(24^{\prime}\right)$ & $1.3(2)$ \\
\hline$C\left(3^{\prime}\right)-C\left(4^{\prime}\right)-C\left(5^{\prime}\right)-C\left(6^{\prime}\right)$ & $112.59(15)$ & \multicolumn{2}{|c|}{$C\left(15^{\prime}\right)-C\left(22^{\prime}\right)-C\left(23^{\prime}\right)-C\left(24^{\prime}\right) 179.57(13)$} \\
\hline $\mathrm{O}\left(1^{\prime}\right)-\mathrm{C}\left(4^{\prime}\right)-\mathrm{C}\left(6^{\prime}\right)-\mathrm{C}\left(5^{\prime}\right)$ & $107.19(14)$ & $C\left(22^{\prime}\right)-C\left(23^{\prime}\right)-C\left(24^{\prime}\right)-C\left(25^{\prime}\right)$ & $-1.4(2)$ \\
\hline$C\left(3^{\prime}\right)-C\left(4^{\prime}\right)-C\left(6^{\prime}\right)-C\left(5^{\prime}\right)$ & $-110.23(15)$ & $C\left(23^{\prime}\right)-C\left(24^{\prime}\right)-C\left(25^{\prime}\right)-C\left(26^{\prime}\right)$ & $0.4(2)$ \\
\hline $\mathrm{O}\left(4^{\prime}\right)-\mathrm{C}\left(5^{\prime}\right)-\mathrm{C}\left(6^{\prime}\right)-\mathrm{C}\left(4^{\prime}\right)$ & -109.2 & $C\left(24^{\prime}\right)-C\left(25^{\prime}\right)-C\left(26^{\prime}\right)-C\left(27^{\prime}\right)$ & $0.7(2)$ \\
\hline$N\left(1^{\prime}\right)-C\left(7^{\prime}\right)-C\left(8^{\prime}\right)-C\left(9^{\prime}\right)$ & $-0.4(2)$ & $C\left(25^{\prime}\right)-C\left(26^{\prime}\right)-C\left(27^{\prime}\right)-C\left(22^{\prime}\right)$ & $-0.8(2)$ \\
\hline $\mathrm{C}\left(7^{\prime}\right)-\mathrm{C}\left(8^{\prime}\right)-\mathrm{C}\left(9^{\prime}\right)-\mathrm{O}\left(6^{\prime}\right)$ & $-179.85(15)$ & $C\left(23^{\prime}\right)-C\left(22^{\prime}\right)-C\left(27^{\prime}\right)-C\left(26^{\prime}\right)$ & $-0.1(2)$ \\
\hline $\mathrm{C}\left(7^{\prime}\right)-\mathrm{C}\left(8^{\prime}\right)-\mathrm{C}\left(9^{\prime}\right)-\mathrm{N}\left(2^{\prime}\right)$ & $-0.3(2)$ & \multicolumn{2}{|c|}{$C\left(15^{\prime}\right)-C\left(22^{\prime}\right)-C\left(27^{\prime}\right)-C\left(26^{\prime}\right)-178.47(13)$} \\
\hline $\mathrm{O}\left(5^{\prime}\right)-\mathrm{C}\left(11^{\prime}\right)-\mathrm{C}\left(12^{\prime}\right)-\mathrm{C}\left(14^{\prime}\right)$ & $9.6(2)$ & \multirow{2}{*}{$\begin{array}{l}N\left(1^{\prime}\right)-C\left(1^{\prime}\right)-O\left(1^{\prime}\right)-C\left(4^{\prime}\right) \\
C\left(2^{\prime}\right)-C\left(1^{\prime}\right)-O\left(1^{\prime}\right)-C\left(4^{\prime}\right)\end{array}$} & $-102.44(12)$ \\
\hline \multicolumn{2}{|c|}{$\mathrm{O}\left(4^{\prime}\right)-\mathrm{C}\left(11^{\prime}\right)-\mathrm{C}\left(12^{\prime}\right)-\mathrm{C}\left(14^{\prime}\right)-170.25(15)$} & & $22.84(14)$ \\
\hline $\mathrm{O}\left(5^{\prime}\right)-\mathrm{C}\left(11^{\prime}\right)-\mathrm{C}\left(12^{\prime}\right)-\mathrm{C}\left(13^{\prime}\right)$ & $134.65(18)$ & $\mathrm{C}\left(5^{\prime}\right)-\mathrm{C}\left(4^{\prime}\right)-\mathrm{O}\left(1^{\prime}\right)-\mathrm{C}\left(1^{\prime}\right)$ & $-168.12(12)$ \\
\hline$O\left(4^{\prime}\right)-C\left(11^{\prime}\right)-C\left(12^{\prime}\right)-C\left(13^{\prime}\right)$ & $-45.20(19)$ & $\mathrm{C}\left(3^{\prime}\right)-\mathrm{C}\left(4^{\prime}\right)-\mathrm{O}\left(1^{\prime}\right)-\mathrm{C}\left(1^{\prime}\right)$ & $-25.25(14)$ \\
\hline$O\left(3^{\prime}\right)-C\left(15^{\prime}\right)-C\left(16^{\prime}\right)-C\left(21^{\prime}\right)$ & $126.68(13)$ & $\mathrm{C}\left(6^{\prime}\right)-\mathrm{C}\left(4^{\prime}\right)-\mathrm{O}\left(1^{\prime}\right)-\mathrm{C}\left(1^{\prime}\right)$ & $122.19(13)$ \\
\hline$O\left(2^{\prime}\right)-C\left(15^{\prime}\right)-C\left(16^{\prime}\right)-C\left(21^{\prime}\right)$ & $12.75(17)$ & $\mathrm{O}\left(3^{\prime}\right)-\mathrm{C}\left(15^{\prime}\right)-\mathrm{O}\left(2^{\prime}\right)-\mathrm{C}\left(2^{\prime}\right)$ & $38.36(13)$ \\
\hline$C\left(22^{\prime}\right)-C\left(15^{\prime}\right)-C\left(16^{\prime}\right)-C\left(21^{\prime}\right)$ & )-109.96(15) & $\mathrm{C}\left(16^{\prime}\right)-\mathrm{C}\left(15^{\prime}\right)-\mathrm{O}\left(2^{\prime}\right)-\mathrm{C}\left(2^{\prime}\right)$ & $155.20(11)$ \\
\hline$O\left(3^{\prime}\right)-C\left(15^{\prime}\right)-C\left(16^{\prime}\right)-C\left(17^{\prime}\right)$ & $-53.79(17)$ & $\mathrm{C}\left(22^{\prime}\right)-\mathrm{C}\left(15^{\prime}\right)-\mathrm{O}\left(2^{\prime}\right)-\mathrm{C}\left(2^{\prime}\right)$ & $-81.32(13)$ \\
\hline$O\left(2^{\prime}\right)-C\left(15^{\prime}\right)-C\left(16^{\prime}\right)-C\left(17^{\prime}\right)-$ & $-167.73(13)$ & $C\left(1^{\prime}\right)-C\left(2^{\prime}\right)-O\left(2^{\prime}\right)-C\left(15^{\prime}\right)$ & $-132.76(11)$ \\
\hline$C\left(22^{\prime}\right)-C\left(15^{\prime}\right)-C\left(16^{\prime}\right)-C\left(17^{\prime}\right)$ & ) $69.56(17)$ & $\mathrm{C}\left(3^{\prime}\right)-\mathrm{C}\left(2^{\prime}\right)-\mathrm{O}\left(2^{\prime}\right)-\mathrm{C}\left(15^{\prime}\right)$ & $-21.41(13)$ \\
\hline$C\left(21^{\prime}\right)-C\left(16^{\prime}\right)-C\left(17^{\prime}\right)-C\left(18^{\prime}\right)$ & $-0.3(2)$ & $\mathrm{O}\left(2^{\prime}\right)-\mathrm{C}\left(15^{\prime}\right)-\mathrm{O}\left(3^{\prime}\right)-\mathrm{C}\left(3^{\prime}\right)$ & $-39.95(13)$ \\
\hline$C\left(15^{\prime}\right)-C\left(16^{\prime}\right)-C\left(17^{\prime}\right)-C\left(18^{\prime}\right)$ & )$-179.80(13)$ & $C\left(16^{\prime}\right)-C\left(15^{\prime}\right)-O\left(3^{\prime}\right)-C\left(3^{\prime}\right)$ & $-156.34(11)$ \\
\hline$C\left(16^{\prime}\right)-C\left(17^{\prime}\right)-C\left(18^{\prime}\right)-C\left(19^{\prime}\right)$ & $-0.1(2)$ & $\mathrm{C}\left(22^{\prime}\right)-\mathrm{C}\left(15^{\prime}\right)-\mathrm{O}\left(3^{\prime}\right)-\mathrm{C}\left(3^{\prime}\right)$ & $79.64(13)$ \\
\hline$C\left(17^{\prime}\right)-C\left(18^{\prime}\right)-C\left(19^{\prime}\right)-C\left(20^{\prime}\right)$ & ) $0.3(3)$ & $\mathrm{C}\left(4^{\prime}\right)-\mathrm{C}\left(3^{\prime}\right)-\mathrm{O}\left(3^{\prime}\right)-\mathrm{C}\left(15^{\prime}\right)$ & $136.75(11)$ \\
\hline$C\left(18^{\prime}\right)-C\left(19^{\prime}\right)-C\left(20^{\prime}\right)-C\left(21^{\prime}\right)$ & $0.0(3)$ & $\mathrm{C}\left(2^{\prime}\right)-\mathrm{C}\left(3^{\prime}\right)-\mathrm{O}\left(3^{\prime}\right)-\mathrm{C}\left(15^{\prime}\right)$ & $25.38(13)$ \\
\hline$C\left(17^{\prime}\right)-C\left(16^{\prime}\right)-C\left(21^{\prime}\right)-C\left(20^{\prime}\right)$ & $0.5(2)$ & $\mathrm{O}\left(5^{\prime}\right)-\mathrm{C}\left(11^{\prime}\right)-\mathrm{O}\left(4^{\prime}\right)-\mathrm{C}\left(5^{\prime}\right)$ & $-0.8(2)$ \\
\hline$C\left(15^{\prime}\right)-C\left(16^{\prime}\right)-C\left(21^{\prime}\right)-C\left(20^{\prime}\right)$ & )$-179.95(14)$ & $\mathrm{C}\left(12^{\prime}\right)-\mathrm{C}\left(11^{\prime}\right)-\mathrm{O}\left(4^{\prime}\right)-\mathrm{C}\left(5^{\prime}\right)$ & $179.02(12)$ \\
\hline
\end{tabular}




\begin{tabular}{lclc}
$\mathrm{C}\left(4^{\prime}\right)-\mathrm{C}\left(5^{\prime}\right)-\mathrm{O}\left(4^{\prime}\right)-\mathrm{C}\left(11^{\prime}\right)$ & $101.66(15)$ & $\mathrm{O}\left(1^{\prime}\right)-\mathrm{C}\left(1^{\prime}\right)-\mathrm{N}\left(1^{\prime}\right)-\mathrm{C}\left(7^{\prime}\right)$ & $-92.85(14)$ \\
$\mathrm{C}\left(6^{\prime}\right)-\mathrm{C}\left(5^{\prime}\right)-\mathrm{O}\left(4^{\prime}\right)-\mathrm{C}\left(11^{\prime}\right)$ & $169.23(12)$ & $\mathrm{C}\left(2^{\prime}\right)-\mathrm{C}\left(1^{\prime}\right)-\mathrm{N}\left(1^{\prime}\right)-\mathrm{C}\left(7^{\prime}\right)$ & $145.31(13)$ \\
$\mathrm{C}\left(8^{\prime}\right)-\mathrm{C}\left(7^{\prime}\right)-\mathrm{N}\left(1^{\prime}\right)-\mathrm{C}\left(10^{\prime}\right)$ & $4.2(2)$ & $\mathrm{O}\left(1^{\prime}\right)-\mathrm{C}\left(1^{\prime}\right)-\mathrm{N}\left(1^{\prime}\right)-\mathrm{C}\left(10^{\prime}\right)$ & $79.05(15)$ \\
$\mathrm{C}\left(8^{\prime}\right)-\mathrm{C}\left(7^{\prime}\right)-\mathrm{N}\left(1^{\prime}\right)-\mathrm{C}\left(1^{\prime}\right)$ & $176.10(14)$ & $\mathrm{C}\left(2^{\prime}\right)-\mathrm{C}\left(1^{\prime}\right)-\mathrm{N}\left(1^{\prime}\right)-\mathrm{C}\left(10^{\prime}\right)$ & $-42.80(17)$ \\
$\mathrm{O}\left(7^{\prime}\right)-\mathrm{C}\left(10^{\prime}\right)-\mathrm{N}\left(1^{\prime}\right)-\mathrm{C}\left(7^{\prime}\right)$ & $173.57(13)$ & $\mathrm{O}\left(7^{\prime}\right)-\mathrm{C}\left(10^{\prime}\right)-\mathrm{N}\left(2^{\prime}\right)-\mathrm{C}\left(9^{\prime}\right)$ & $-173.75(14)$ \\
$\mathrm{N}\left(2^{\prime}\right)-\mathrm{C}\left(10^{\prime}\right)-\mathrm{N}\left(1^{\prime}\right)-\mathrm{C}\left(7^{\prime}\right)$ & $-6.97(19)$ & $\mathrm{N}\left(1^{\prime}\right)-\mathrm{C}\left(10^{\prime}\right)-\mathrm{N}\left(2^{\prime}\right)-\mathrm{C}\left(9^{\prime}\right)$ & $6.8(2)$ \\
$\mathrm{O}\left(7^{\prime}\right)-\mathrm{C}\left(10^{\prime}\right)-\mathrm{N}\left(1^{\prime}\right)-\mathrm{C}\left(1^{\prime}\right)$ & $1.7(2)$ & $\mathrm{O}\left(6^{\prime}\right)-\mathrm{C}\left(9^{\prime}\right)-\mathrm{N}\left(2^{\prime}\right)-\mathrm{C}\left(10^{\prime}\right)$ & $176.41(14)$ \\
$\mathrm{N}\left(2^{\prime}\right)-\mathrm{C}\left(10^{\prime}\right)-\mathrm{N}\left(1^{\prime}\right)-\mathrm{C}\left(1^{\prime}\right)$ & $-178.80(12)$ & $\mathrm{C}\left(8^{\prime}\right)-\mathrm{C}\left(9^{\prime}\right)-\mathrm{N}\left(2^{\prime}\right)-\mathrm{C}\left(10^{\prime}\right)$ & $-3.2(2)$ \\
& & & \\
\hline
\end{tabular}


Table S16. Hydrogen Bonds [ $\AA$ and $\left.{ }^{\circ}\right]$.

\begin{tabular}{lcccc}
\hline $\mathrm{D}-\mathrm{H} \ldots \mathrm{A}$ & $\mathrm{d}(\mathrm{D}-\mathrm{H})$ & $\mathrm{d}(\mathrm{H} \ldots \mathrm{A})$ & $\mathrm{d}(\mathrm{D} \ldots \mathrm{A})$ & $<(\mathrm{DHA})$ \\
\hline $\mathrm{N}(2)-\mathrm{H}(02) \ldots \mathrm{O}\left(6^{\prime}\right)$ & $0.89(2)$ & $1.92(2)$ & $2.7947(17)$ & $168.2(19)$ \\
$\mathrm{N}\left(2^{\prime}\right)-\mathrm{H}\left(02^{\prime}\right) \ldots \mathrm{O}(6)$ & $0.854(19)$ & $1.98(2)$ & $2.8260(17)$ & $171.0(17)$ \\
& & & & \\
$\mathrm{C}(3)-\mathrm{H}(3) \ldots \mathrm{O}\left(7^{\prime}\right) \# 1$ & 1.00 & 2.35 & $3.2798(17)$ & 154.5 \\
$\mathrm{C}(5)-\mathrm{H}(5) \ldots \mathrm{O}(6) \# 1$ & 1.00 & 2.52 & $3.4371(18)$ & 151.5 \\
$\mathrm{C}(6)-\mathrm{H}(6 \mathrm{~A}) \ldots \mathrm{O}\left(7^{\prime}\right) \# 1$ & 0.99 & 2.56 & $3.4183(18)$ & 145.0 \\
$\mathrm{C}(12)-\mathrm{H}(12) \ldots \mathrm{O}\left(4^{\prime}\right) \# 2$ & 1.00 & 2.52 & $3.518(2)$ & 172.7 \\
$\mathrm{C}\left(3^{\prime}\right)-\mathrm{H}\left(3^{\prime}\right) \ldots \mathrm{O}(7) \# 3$ & 1.00 & 2.44 & $3.3072(17)$ & 145.2 \\
$\mathrm{C}\left(5^{\prime}\right)-\mathrm{H}\left(5^{\prime}\right) \ldots \mathrm{O}\left(6^{\prime}\right) \# 3$ & 1.00 & 2.47 & $3.4607(18)$ & 173.2 \\
$\mathrm{C}\left(6^{\prime}\right)-\mathrm{H}\left(6 \mathrm{~B}^{\prime}\right) \ldots \mathrm{O}(7) \# 3$ & 0.99 & 2.47 & $3.3303(18)$ & 145.0 \\
$\mathrm{C}\left(8^{\prime}\right)-\mathrm{H}\left(8^{\prime}\right) \ldots \mathrm{O}\left(5^{\prime}\right) \# 1$ & 0.95 & 2.59 & $3.4719(19)$ & 154.7 \\
$\mathrm{C}\left(12^{\prime}\right)-\mathrm{H}\left(12^{\prime}\right) \ldots \mathrm{O}(4) \# 4$ & 1.00 & 2.63 & $3.589(2)$ & 159.5 \\
& & & & \\
\hline
\end{tabular}

Symmetry transformations used to generate equivalent atoms:

$\# 1 \mathrm{x}+1, \mathrm{y}, \mathrm{z}$

\#2 $x+1, y-1, z$

\#3 $x-1, y, z$

$\# 4 x-1, y+1, z$ 


\section{X-Ray Data for Compound 17}

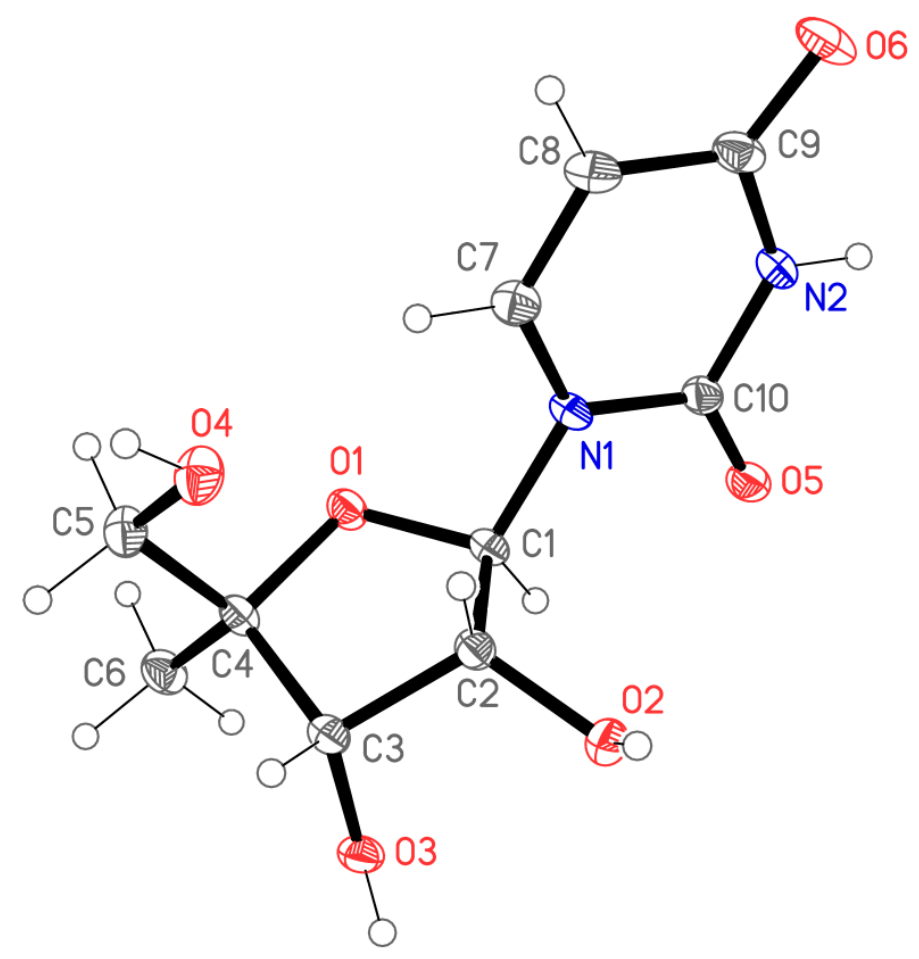

Figure S75. Structure of compound $\mathbf{1 7}$ in the crystal. Thermal ellipsoid plots (all at the $50 \%$ probability level).

Crystallization was performed by slow evaporation of methanol from a vial. 


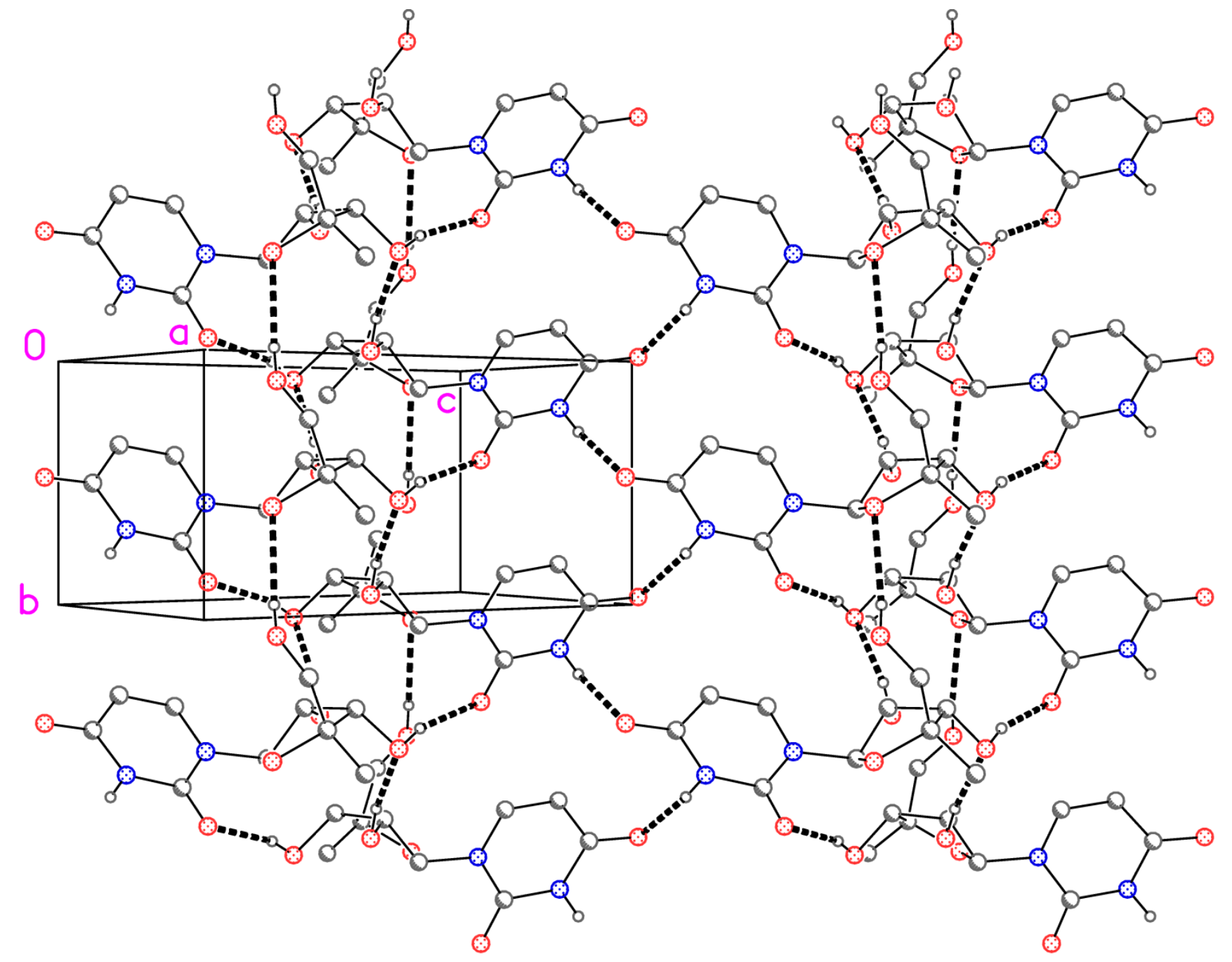

Figure S76. Packing diagram of compound 17. Dashed lines indicate hydrogen bonds (for details see the deposited CIF file). The molecules associate to form broad layers parallel to $(10 \overline{1})$; the view direction is perpendicular to this plane.

Table S17. Crystal Data and Structure Refinement.

$\begin{array}{ll}\text { Identification code } & \text { knoell } \\ \text { Empirical formula } & \mathrm{C}_{10} \mathrm{H}_{14} \mathrm{~N}_{2} \mathrm{O}_{6} \\ \text { Formula weight } & 258.23 \\ \text { Temperature } & 100(2) \mathrm{K} \\ \text { Wavelength } & 1.54184 \AA \\ \text { Crystal system } & \text { Monoclinic } \\ \text { Space group } & \mathrm{P} 2{ }_{1}\end{array}$




\begin{tabular}{|c|c|c|}
\hline \multirow[t]{3}{*}{ Unit cell dimensions } & $a=8.35326(14) \AA$ & $\alpha=90^{\circ}$ \\
\hline & $\mathrm{b}=5.76025(11) \AA$ & $\beta=95.1416(16)^{\circ}$ \\
\hline & $c=11.5435(2) \AA$ & $Y=90^{\circ}$ \\
\hline Volume & \multicolumn{2}{|l|}{$553.203(17) \AA^{3}$} \\
\hline Z & \multicolumn{2}{|l|}{2} \\
\hline Density (calculated) & \multicolumn{2}{|l|}{$1.550 \mathrm{Mg} / \mathrm{m}^{3}$} \\
\hline Absorption coefficient & \multicolumn{2}{|l|}{$1.113 \mathrm{~mm}^{-1}$} \\
\hline$F(000)$ & \multicolumn{2}{|l|}{272} \\
\hline Crystal size & \multicolumn{2}{|c|}{$0.12 \times 0.10 \times 0.08 \mathrm{~mm}^{3}$} \\
\hline Theta range for data collection & \multicolumn{2}{|l|}{3.845 to $77.573^{\circ}$} \\
\hline Index ranges & \multicolumn{2}{|c|}{$-10<=\mathrm{h}<=10,-7<=\mathrm{k}<=7,-14<=\mathrm{k}<=14$} \\
\hline Reflections collected & \multicolumn{2}{|l|}{22406} \\
\hline Independent reflections & \multicolumn{2}{|c|}{$2297[\mathrm{R}$ (int) $=0.0310]$} \\
\hline Completeness to theta $=77.000^{\circ}$ & \multicolumn{2}{|l|}{$99.1 \%$} \\
\hline Absorption correction & \multicolumn{2}{|c|}{ Semi-empirical from equivalents } \\
\hline Max. and min. transmission & \multicolumn{2}{|c|}{1.00000 and 0.89496} \\
\hline Refinement method & \multicolumn{2}{|c|}{ Full-matrix least-squares on $\mathrm{F}^{2}$} \\
\hline Data / restraints / parameters & \multicolumn{2}{|l|}{$2297 / 1 / 181$} \\
\hline Goodness-of-fit on $\mathrm{F}^{2}$ & \multicolumn{2}{|l|}{1.065} \\
\hline Final $R$ indices [l>2sigma(I)] & \multicolumn{2}{|c|}{$\mathrm{R} 1=0.0221, \mathrm{wR} 2=0.0578$} \\
\hline $\mathrm{R}$ indices (all data) & \multicolumn{2}{|c|}{$\mathrm{R} 1=0.0222, \mathrm{wR} 2=0.0579$} \\
\hline Absolute structure parameter & \multicolumn{2}{|l|}{$-0.03(5)$} \\
\hline Extinction coefficient & \multicolumn{2}{|l|}{$0.0060(12)$} \\
\hline Largest diff. peak and hole & \multicolumn{2}{|c|}{0.186 and -0.159 e. $\AA^{-3}$} \\
\hline
\end{tabular}


Table S18. Atomic Coordinates $\left(x 1^{4}\right)$ and Equivalent Isotropic Displacement Parameters $\left(\AA^{2} \times 10^{3}\right) . \cup(e q)$ is Defined as one Third of the Trace of the Orthogonalized Uij Tensor.

\begin{tabular}{|c|c|c|c|c|}
\hline & $x$ & $y$ & $z$ & $\mathrm{U}(\mathrm{eq})$ \\
\hline$C(1)$ & $5569(2)$ & $6022(3)$ & $2951(1)$ & $14(1)$ \\
\hline$C(2)$ & $5513(2)$ & $4072(3)$ & $3839(1)$ & $14(1)$ \\
\hline$C(3)$ & $7263(2)$ & $3997(3)$ & $4377(1)$ & $14(1)$ \\
\hline$C(4)$ & $8228(2)$ & $4625(3)$ & $3343(1)$ & $15(1)$ \\
\hline$C(5)$ & $8773(2)$ & $2474(3)$ & $2722(2)$ & $19(1)$ \\
\hline$C(6)$ & $9667(2)$ & 6192(3) & $3653(1)$ & $19(1)$ \\
\hline$C(7)$ & $4389(2)$ & $3882(3)$ & $1243(1)$ & $18(1)$ \\
\hline$C(8)$ & $3242(2)$ & $3456(3)$ & $374(1)$ & $20(1)$ \\
\hline $\mathrm{C}(9)$ & $1884(2)$ & $4987(3)$ & $208(1)$ & $19(1)$ \\
\hline$C(10)$ & $3091(2)$ & $7360(3)$ & $1848(1)$ & $15(1)$ \\
\hline $\mathrm{O}(1)$ & $7099(1)$ & $5895(2)$ & $2516(1)$ & $15(1)$ \\
\hline $\mathrm{O}(2)$ & $4369(1)$ & $4581(2)$ & $4627(1)$ & $16(1)$ \\
\hline $\mathrm{O}(3)$ & $7534(1)$ & $5667(2)$ & $5292(1)$ & $17(1)$ \\
\hline $\mathrm{O}(4)$ & $7433(1)$ & $955(2)$ & $2490(1)$ & $20(1)$ \\
\hline $\mathrm{O}(5)$ & $2986(1)$ & $9046(2)$ & $2478(1)$ & $19(1)$ \\
\hline $\mathrm{O}(6)$ & $725(1)$ & $4753(3)$ & $-521(1)$ & $24(1)$ \\
\hline$N(1)$ & $4334(2)$ & $5771(3)$ & $1969(1)$ & $15(1)$ \\
\hline $\mathrm{N}(2)$ & $1941(2)$ & 6886(3) & $945(1)$ & $18(1)$ \\
\hline
\end{tabular}


Table S19. Bond Lengths $[\AA ̊]$ and Angles $\left[^{\circ}\right]$.

\begin{tabular}{|c|c|c|c|}
\hline $\mathrm{C}(1)-\mathrm{O}(1)$ & $1.4170(18)$ & $\mathrm{C}(5)-\mathrm{O}(4)$ & $1.427(2)$ \\
\hline$C(1)-N(1)$ & $1.4703(17)$ & $\mathrm{C}(7)-\mathrm{C}(8)$ & $1.346(2)$ \\
\hline$C(1)-C(2)$ & $1.524(2)$ & $\mathrm{C}(7)-\mathrm{N}(1)$ & $1.377(2)$ \\
\hline $\mathrm{C}(2)-\mathrm{O}(2)$ & $1.4084(18)$ & $\mathrm{C}(8)-\mathrm{C}(9)$ & $1.436(2)$ \\
\hline$C(2)-C(3)$ & $1.5363(19)$ & $\mathrm{C}(9)-\mathrm{O}(6)$ & $1.232(2)$ \\
\hline $\mathrm{C}(3)-\mathrm{O}(3)$ & $1.4323(18)$ & $\mathrm{C}(9)-\mathrm{N}(2)$ & $1.384(2)$ \\
\hline$C(3)-C(4)$ & $1.542(2)$ & $C(10)-O(5)$ & $1.221(2)$ \\
\hline $\mathrm{C}(4)-\mathrm{O}(1)$ & $1.4751(17)$ & $C(10)-N(2)$ & $1.380(2)$ \\
\hline$C(4)-C(6)$ & $1.520(2)$ & $C(10)-N(1)$ & $1.381(2)$ \\
\hline$C(4)-C(5)$ & $1.522(2)$ & & \\
\hline$O(1)-C(1)-N(1)$ & $108.31(11)$ & $\mathrm{O}(4)-\mathrm{C}(5)-\mathrm{C}(4)$ & $108.98(13)$ \\
\hline $\mathrm{O}(1)-\mathrm{C}(1)-\mathrm{C}(2)$ & $106.46(12)$ & $C(8)-C(7)-N(1)$ & $122.48(15)$ \\
\hline$N(1)-C(1)-C(2)$ & $112.63(13)$ & $C(7)-C(8)-C(9)$ & $119.24(16)$ \\
\hline $\mathrm{O}(2)-\mathrm{C}(2)-\mathrm{C}(1)$ & $110.13(13)$ & $\mathrm{O}(6)-\mathrm{C}(9)-\mathrm{N}(2)$ & $119.19(16)$ \\
\hline $\mathrm{O}(2)-\mathrm{C}(2)-\mathrm{C}(3)$ & $114.91(12)$ & $\mathrm{O}(6)-\mathrm{C}(9)-\mathrm{C}(8)$ & $125.97(17)$ \\
\hline$C(1)-C(2)-C(3)$ & $101.98(12)$ & $N(2)-C(9)-C(8)$ & $114.84(14)$ \\
\hline $\mathrm{O}(3)-\mathrm{C}(3)-\mathrm{C}(2)$ & $111.16(13)$ & $\mathrm{O}(5)-\mathrm{C}(10)-\mathrm{N}(2)$ & $121.61(15)$ \\
\hline $\mathrm{O}(3)-\mathrm{C}(3)-\mathrm{C}(4)$ & $110.73(13)$ & $\mathrm{O}(5)-\mathrm{C}(10)-\mathrm{N}(1)$ & $124.11(14)$ \\
\hline$C(2)-C(3)-C(4)$ & $103.01(11)$ & $N(2)-C(10)-N(1)$ & $114.28(14)$ \\
\hline $\mathrm{O}(1)-\mathrm{C}(4)-\mathrm{C}(6)$ & $107.70(13)$ & $C(1)-O(1)-C(4)$ & $109.91(11)$ \\
\hline $\mathrm{O}(1)-\mathrm{C}(4)-\mathrm{C}(5)$ & $107.43(12)$ & $C(7)-N(1)-C(10)$ & $121.90(13)$ \\
\hline$C(6)-C(4)-C(5)$ & 109.32(13) & $C(7)-N(1)-C(1)$ & $119.18(13)$ \\
\hline $\mathrm{O}(1)-\mathrm{C}(4)-\mathrm{C}(3)$ & $105.61(11)$ & $C(10)-N(1)-C(1)$ & $118.78(13)$ \\
\hline$C(6)-C(4)-C(3)$ & $114.51(12)$ & $C(10)-N(2)-C(9)$ & $127.13(15)$ \\
\hline$C(5)-C(4)-C(3)$ & $111.89(14)$ & & \\
\hline
\end{tabular}


Table S20. Torsion Angles [ $\left.{ }^{\circ}\right]$.

\begin{tabular}{|c|c|c|c|}
\hline $\mathrm{O}(1)-\mathrm{C}(1)-\mathrm{C}(2)-\mathrm{O}(2)$ & $156.99(12)$ & $N(1)-C(1)-O(1)-C(4)$ & $-142.43(13)$ \\
\hline$N(1)-C(1)-C(2)-O(2)$ & $-84.45(15)$ & $C(2)-C(1)-O(1)-C(4)$ & $-21.07(15)$ \\
\hline $\mathrm{O}(1)-\mathrm{C}(1)-\mathrm{C}(2)-\mathrm{C}(3)$ & $34.55(15)$ & $\mathrm{C}(6)-\mathrm{C}(4)-\mathrm{O}(1)-\mathrm{C}(1)$ & $-124.09(14)$ \\
\hline$N(1)-C(1)-C(2)-C(3)$ & $153.11(12)$ & $C(5)-C(4)-O(1)-C(1)$ & $118.26(13)$ \\
\hline $\mathrm{O}(2)-\mathrm{C}(2)-\mathrm{C}(3)-\mathrm{O}(3)$ & $-34.54(19)$ & $C(3)-C(4)-O(1)-C(1)$ & $-1.31(16)$ \\
\hline$C(1)-C(2)-C(3)-O(3)$ & $84.56(14)$ & $C(8)-C(7)-N(1)-C(10)$ & $-0.1(2)$ \\
\hline $\mathrm{O}(2)-\mathrm{C}(2)-\mathrm{C}(3)-\mathrm{C}(4)$ & $-153.17(14)$ & $C(8)-C(7)-N(1)-C(1)$ & $175.53(15)$ \\
\hline$C(1)-C(2)-C(3)-C(4)$ & $-34.06(15)$ & $\mathrm{O}(5)-\mathrm{C}(10)-\mathrm{N}(1)-\mathrm{C}(7)$ & $179.27(15)$ \\
\hline $\mathrm{O}(3)-\mathrm{C}(3)-\mathrm{C}(4)-\mathrm{O}(1)$ & $-96.31(14)$ & $N(2)-C(10)-N(1)-C(7)$ & $-0.4(2)$ \\
\hline $\mathrm{C}(2)-\mathrm{C}(3)-\mathrm{C}(4)-\mathrm{O}(1)$ & $22.62(16)$ & $\mathrm{O}(5)-\mathrm{C}(10)-\mathrm{N}(1)-\mathrm{C}(1)$ & $3.7(2)$ \\
\hline $\mathrm{O}(3)-\mathrm{C}(3)-\mathrm{C}(4)-\mathrm{C}(6)$ & $22.01(18)$ & $N(2)-C(10)-N(1)-C(1)$ & $-175.96(13)$ \\
\hline$C(2)-C(3)-C(4)-C(6)$ & $140.93(14)$ & $\mathrm{O}(1)-\mathrm{C}(1)-\mathrm{N}(1)-\mathrm{C}(7)$ & $53.75(18)$ \\
\hline $\mathrm{O}(3)-\mathrm{C}(3)-\mathrm{C}(4)-\mathrm{C}(5)$ & $147.11(13)$ & $C(2)-C(1)-N(1)-C(7)$ & $-63.72(17)$ \\
\hline$C(2)-C(3)-C(4)-C(5)$ & $-93.96(14)$ & $\mathrm{O}(1)-\mathrm{C}(1)-\mathrm{N}(1)-\mathrm{C}(10)$ & $-130.53(14)$ \\
\hline $\mathrm{O}(1)-\mathrm{C}(4)-\mathrm{C}(5)-\mathrm{O}(4)$ & $-66.49(16)$ & $C(2)-C(1)-N(1)-C(10)$ & $112.00(16)$ \\
\hline$C(6)-C(4)-C(5)-O(4)$ & $176.91(13)$ & $\mathrm{O}(5)-\mathrm{C}(10)-\mathrm{N}(2)-\mathrm{C}(9)$ & $-176.85(15)$ \\
\hline $\mathrm{C}(3)-\mathrm{C}(4)-\mathrm{C}(5)-\mathrm{O}(4)$ & $48.99(16)$ & $N(1)-C(10)-N(2)-C(9)$ & $2.8(2)$ \\
\hline$N(1)-C(7)-C(8)-C(9)$ & $-1.6(3)$ & $\mathrm{O}(6)-\mathrm{C}(9)-\mathrm{N}(2)-\mathrm{C}(10)$ & $176.15(15)$ \\
\hline $\mathrm{C}(7)-\mathrm{C}(8)-\mathrm{C}(9)-\mathrm{O}(6)$ & $-177.00(16)$ & $C(8)-C(9)-N(2)-C(10)$ & $-4.4(2)$ \\
\hline $\mathrm{C}(7)-\mathrm{C}(8)-\mathrm{C}(9)-\mathrm{N}(2)$ & $3.6(2)$ & & \\
\hline
\end{tabular}


Table S21. Hydrogen Bonds [ $\AA$ and $\left.{ }^{\circ}\right]$.

\begin{tabular}{lcccc}
\hline $\mathrm{D}-\mathrm{H} \ldots \mathrm{A}$ & $\mathrm{d}(\mathrm{D}-\mathrm{H})$ & $\mathrm{d}(\mathrm{H} \ldots \mathrm{A})$ & $\mathrm{d}(\mathrm{D} \ldots \mathrm{A})$ & $<(\mathrm{DHA})$ \\
\hline $\mathrm{O}(2)-\mathrm{H}(02) \ldots \mathrm{O}(3) \# 1$ & $0.88(3)$ & $1.89(3)$ & $2.7647(17)$ & $172(2)$ \\
$\mathrm{O}(3)-\mathrm{H}(03) \ldots \mathrm{O}(5) \# 1$ & $0.88(3)$ & $1.95(3)$ & $2.8084(16)$ & $164(2)$ \\
$\mathrm{O}(4)-\mathrm{H}(04) \ldots \mathrm{O}(1) \# 2$ & $0.83(3)$ & $2.28(3)$ & $2.9281(19)$ & $135(2)$ \\
$\mathrm{O}(4)-\mathrm{H}(04) \ldots \mathrm{O}(6) \# 3$ & $0.83(3)$ & $2.48(2)$ & $2.9395(17)$ & $116(2)$ \\
$\mathrm{N}(2)-\mathrm{H}(01) \ldots \mathrm{O}(6) \# 4$ & $0.89(3)$ & $1.90(3)$ & $2.7801(18)$ & $174(2)$ \\
$\mathrm{C}(7)-\mathrm{H}(7) \ldots \mathrm{O}(4)$ & 0.95 & 2.39 & $3.275(2)$ & 154.5 \\
& & & & \\
\hline
\end{tabular}

Symmetry transformations used to generate equivalent atoms:

$\# 1-x+1, y-1 / 2,-z+1$

$\# 2 x, y-1, z$

$\# 3-x+1, y-1 / 2,-z$

$\# 4-x, y+1 / 2,-z$ 


\section{Antiviral Investigations}

7

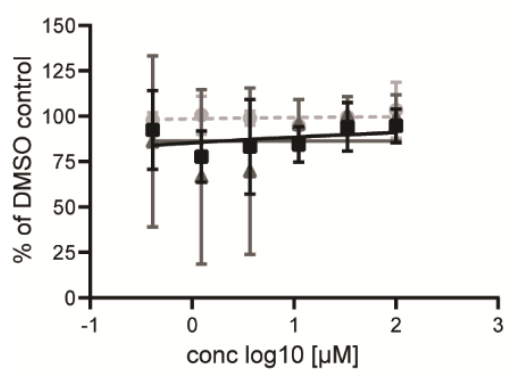

17

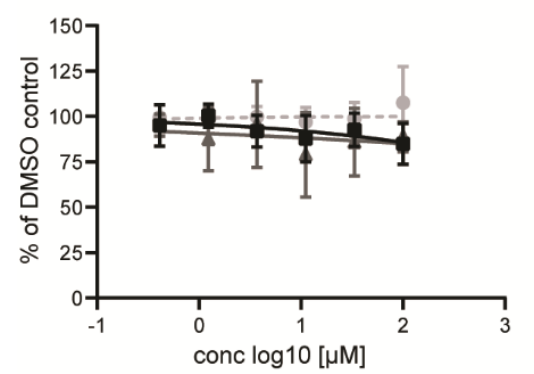

infection round I
8

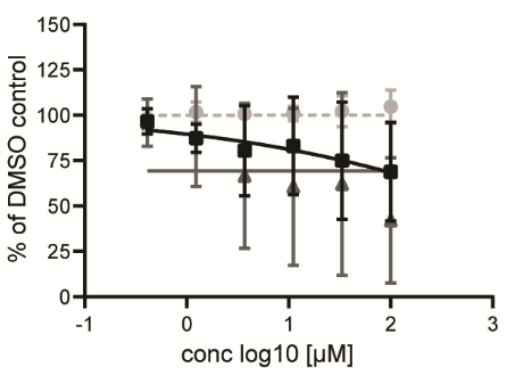

18

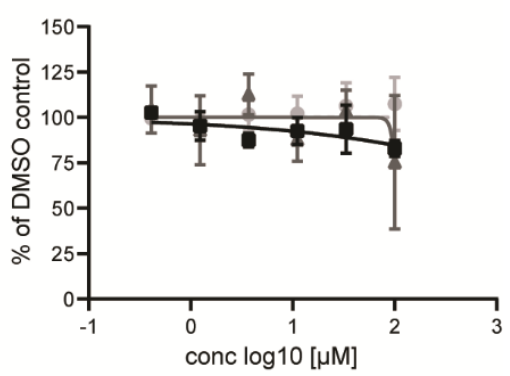

-๑ cell viability

Figure S77. Antiviral activity and cell toxicity of compounds 7, 8, 17 and 18. Mean and standard deviations of three independent experiments are shown. 


\section{Literature}

[1] Sheldrick, G. M. Crystal structure refinement with SHELXL. Acta Cryst. 2015, C71, 3-8. 